\title{
A TOPOLOGICAL ANALOGUE OF MOSTOW'S RIGIDITY THEOREM
}

\author{
F. T. FARRELL AND L. E. JONES
}

\section{CONTENTS}

0 . Introduction

1. Geometric preliminaries

2. Metric properties of $F M$

3. Leaves of small injectivity radius

4. The transferred surgery obstruction

5. Splitting the transferred map

6. Two splitting theorems

7. The asymptotic transfer

8. Completion of the proof for Theorem 5.5

9. Computation of surgery groups

10. Applications

A. Appendix

\section{INTRODUCTION}

Three types of manifolds, spherical, flat, and hyperbolic, are paradigms of geometric behavior. These are the Riemannian manifolds of constant positive, zero, and negative sectional curvatures, respectively. (The positive and negative sectional curvatures may be assumed, after scaling, to be +1 and -1 .) There is an equivalent synthetic geometric definition of them in terms of coordinate charts for a manifold $M$. Namely, a spherical, flat, or hyperbolic structure on $M$ is an atlas of coordinate charts $\left\{\left(U_{\alpha}, f_{\alpha}\right) \mid \alpha \in \mathscr{I}\right\}$ for $M$, where each $f_{\alpha x}\left(U_{\alpha x}\right)$ is an open subset of the (unit) sphere $S^{n}$, Euclidean space $R^{n}$, or (real) hyperbolic space $H^{n}$, respectively, such that

$$
f_{\gamma} \circ f_{\alpha}^{-1}: f_{\alpha}\left(U_{\alpha} \cap U_{\gamma}\right) \rightarrow f_{\gamma}\left(U_{\alpha} \cap U_{\gamma}\right)
$$

Presented at the Special Session on Geometric Methods in Group Theory at the sectional meeting of the American Mathematical Society in College Park, Maryland, April 1988; received by the editors July 20, 1988.

1980 Mathematics Subject Classification (1985 Revision). Primary 18F25, 22E40, 57D50.

Both authors were supported in part by the NSF. 
is the restriction of an isometry of $S^{n}, R^{n}, H^{n}$ (as the case may be) for all pairs $\alpha, \gamma$ in the indexing set $\mathscr{I}$.

Mostow proved [50] the following remarkable uniqueness result for hyperbolic structures.

Mostow's Rigidity Theorem. Let $M$ be a compact (connected) topological manifold whose dimension is greater than 2 . Then $M$ can support at most one hyperbolic structure; i.e., if $M_{1}$ and $M_{2}$ are hyperbolic manifolds that are homeomorphic to $M$, then $M_{1}$ and $M_{2}$ are isometrically equivalent. In fact, this conclusion is implied by the weaker assumption that $\pi_{1} M_{1}$ and $\pi_{1} M_{2}$ are isomorphic groups, and any isomorphism is induced, up to conjugacy, by an isometry.

We prove, in this paper, the following existence theorem for hyperbolic structures. (We sometimes paraphrase results in this section.) In its statement, $O(1, m)$ denotes the Lie group consisting of all square $(m+1) \times(m+1)$ matrices $A$ with real number entries that satisfy the equation $A D A^{t}=D$, where $D$ is the diagonal matrix with $D_{11}=-1$ and $D_{i i}=1$ when $i>1$.

Corollary 10.4. A compact (connected) topological manifold $M^{m}$, whose dimension $m$ is different from 3 and 4 , supports a hyperbolic structure if and only if

(i) $\pi_{1} M$ is isomorphic to a discrete cocompact subgroup of $O(1, m)$, and

(ii) the universal covering space of $M$ is contractible, i.e., $\pi_{j} M=0$ for all $j>1$.

It is standard terminology to call a connected manifold $M$ aspherical if $\pi_{j} M$ vanishes for all $j>1$. If $M_{1}$ and $M_{2}$ are aspherical manifolds, then any isomorphism from $\pi_{1} M_{1}$ to $\pi_{1} M_{2}$ is induced by a homotopy equivalence from $M_{1}$ to $M_{2}$. (This is a well-known consequence of the classical Eilenberg Obstruction Theory.) Using this fact, we deduce Corollary 10.4 from the following result (whose paraphrased statement also includes some other consequences of Theorem 10.1).

Corollary 10.3. Let $M_{1}$ and $M_{2}$ be a pair of compact aspherical manifolds. Then any isomorphism from $\pi_{1} M_{1}$ to $\pi_{1} M_{2}$ is induced (up to conjugacy) by a homeomorphism from $M_{1}$ to $M_{2}$ provided $M_{1}$ has a hyperbolic structure ${ }^{1}$ and the dimension of $M_{1}$ is different from 3 and 4 .

It is well known that if Corollary 10.3 were true when $\operatorname{dim} M_{1}=3$, then the Poincare Conjecture is true. (Because of [44] and [47], the connected sum of $M_{1}^{3}$ with a counterexample to Poincaré's Conjecture would contradict the 3-dimensional statement of Corollary 10.3.) Although we calculate the surgery $L$-groups (cf. Theorem 9.5) for all complete hyperbolic manifolds $M$ (even when $\operatorname{dim} M=3$ or 4 ), surgery theory is not applicable in dimensions 3 or

\footnotetext{
${ }^{1}$ We have recently shown, cf. [66], that Corollary 10.3 remains true under the weaker assumption that $M_{1}$ admits a Riemannian structure whose sectional curvatures are all negative.
} 
4. Hence, we are unable to verify the statement of Corollary 10.3 when the dimension of $M$ is either 3 or 4 .

It is common to impose a completeness condition on a Riemannian manifold $M$, namely, that any pair of points in $M$ lies on a common geodesic. Completeness is implied by compactness; but when $M$ is noncompact, it is an important extra assumption. For instance, the Smale-Hirsch immersion theorem, cf. [36], implies that any connected, noncompact, parallelizable manifold can support all three of these special structures: spherical, flat, and hyperbolic. But the completeness hypothesis imposes stringent constraints on the topology of $M^{m}$. In particular, if $M^{m}$ has a complete constant sectional curvature Riemannian structure, then its universal covering space is homeomorphic (in fact, isometrically equivalent after perhaps a scaling) to $S^{m}, R^{m}$, or $H^{m}$, and $\pi_{1} M$ is isomorphic to a discrete subgroup of the Lie groups $O(m), O(m) \ltimes R^{m}$, or $O(1, m)$, depending on whether the sign of the sectional curvature of $M^{m}$ is positive, zero, or negative (cf. [35]).

There is a second geometric condition (weaker than compactness) that is frequently imposed on a Riemannian manifold $M$, namely, that $M$ have finite volume. Gromov and Margulis (cf. [32]) showed that a complete hyperbolic manifold $M$ with finite volume is the interior of a compact manifold with boundary $\bar{M}$. Furthermore, the boundary $\partial \bar{M}$ supports a flat Riemannian structure. We call $\bar{M}$ the Gromov-Margulis manifold compactification of $M$. Their compactification actually exists when the hyperbolic condition is replaced by the weaker assumption that the sectional curvatures of $M$ all lie in a closed interval of (strictly) negative numbers. For instance, $\bar{M}$ exists for any complete (connected) negatively curved locally symmetric space with finite volume. In this case, each component of $\partial \bar{M}$ is aspherical but need not support a flat Riemannian structure.

Prasad [56] showed that Mostow's rigidity theorem holds when the compactness condition on $M$ is weakened to the assumption that $M$ is complete (connected) and has finite volume.

Mostow-Prasad Rigidity Theorem. Let $M_{1}$ and $M_{2}$ be a pair of complete (connected) hyperbolic manifolds each of which has finite volume. Then any isomorphism of $\pi_{1} M_{1}$ to $\pi_{1} M_{2}$ is induced (up to conjugacy) by an isometry from $M_{1}$ to $M_{2}$, provided $\operatorname{dim} M_{1}>2$.

Recall that a proper map $f: X \rightarrow Y$ between topological spaces is a continuous map such that $f^{-1}(K)$ is compact whenever $K$ is a compact subset of $Y$. The notions of proper homotopy and proper homotopy equivalence are defined in a similar fashion. The next result is our analogue of the Mostow-Prasad rigidity theorem.

Corollary 10.5. Let $M$ be a complete (connected) hyperbolic manifold with finite volume whose dimension is different from 3,4 , and 5 . Let $N$ be any topological manifold that is properly homotopically equivalent to $M$. Then $N$ 
and $M$ are homeomorphic. In fact, any proper homotopy equivalence is properly homotopic to a homeomorphism.

To prove Corollary 10.5 , we first extend the proper homotopy equivalence $f: N \rightarrow M$ to a homotopy equivalence $\bar{f}: \bar{N} \rightarrow \bar{M}$, where $\bar{N}$ is a manifold compactification of $N$. We use Siebenmann's thesis [61] (which requires $\operatorname{dim} M>5$ ) together with [16] to do this. Then we use [21] to homotope $\bar{f}$ so that its restriction to $\partial \bar{N}$ is a homeomorphism. This also requires $\operatorname{dim} M>5$. On the other hand, Freedman and Quinn [31] have recently shown that surgery theory works for 4-dimensional manifolds provided their fundamental groups are virtually poly-Z $\mathbf{Z}$. Hence, Corollary 10.5 is probably true even when $\operatorname{dim} M=5$.

The following result is valid even when $M$ has $\infty$-volume.

Theorem 10.1 (special case). Let $f: N \rightarrow M$ be a proper homotopy equivalence that is a homeomorphism over the complement of a compact subset of $M$, where $M$ is a complete (connected) hyperbolic manifold with $\operatorname{dim} M>4$. Then $f$ is homotopic to a homeomorphism via a homotopy that is constant over the complement of a larger compact subset of $M$.

Our results, stated above, are derived from surgery theory (cf. [64] and [43]), which we make into an effective method by calculating the surgery obstruction groups $L_{n}\left(\pi_{1} M\right)$ when $M$ is a complete (connected) hyperbolic manifold. Our calculation, Theorem 9.5, is the main result of this paper. Although $L_{n}\left(\pi_{1} M\right)$ is algebraically defined, it is accomplished by a motley assortment of techniques, but mainly by a combination of topological control theory with some special geometric and dynamical properties of $M$.

We also combine Theorem 9.5 with our earlier work [25] on the stable pseudoisotopy space of $M$ (as extended in the appendix) to obtain an understanding of the homotopy type of Top $M$ "through a stable range of dimensions" when $M$ is a complete (connected) hyperbolic manifold. (The group of all homeomorphisms of $M$ with compact support is denoted by Top $M$.) Some notation is needed to express a sample of our results. Let $\times \operatorname{Top}\left(S^{1} \times R^{m-1}\right)$ denote the Cartesian product of a countably infinite number of copies of $\operatorname{Top}\left(S^{1} \times R^{m-1}\right)$. In particular, a point in $\times \operatorname{Top}\left(S^{1} \times R^{m-1}\right)$ is a sequence $f_{i}$ of homeomorphisms in $\operatorname{Top}\left(S^{1} \times R^{m-1}\right)$. Then $\bigoplus \operatorname{Top}\left(S^{1} \times R^{m-1}\right)$ denotes the subspace of $\times \operatorname{Top}\left(S^{1} \times R^{m-1}\right)$ consisting of all sequences $f_{i}$ such that $f_{i}$ is the identity homeomorphism except for a finite number of indices $i$. The outer automorphism group of $\pi_{1} M$ is denoted by Out $\pi_{1} M$. There is a canonical homomorphism from Top $M$ to Out $\pi_{1} M$ that associates to a homeomorphism $f$ the automorphism of $\pi_{1} M$ that it induces (this automorphism is well defined up to conjugation). We denote the kernel of this homomorphism by $\operatorname{Top}_{0} M$. We can now state a representative sample of our results about Top $M$.

Corollary 10.13. Let $M^{m}$ be a compact (connected) hyperbolic manifold whose dimension $m$ is greater than 10. Then $\operatorname{Top}_{0} M^{m}$ and $\bigoplus \operatorname{Top}\left(S^{1} \times R^{m-1}\right)$ 
are weakly homotopically equivalent "through a stable range of dimensions"; i.e., there exists a continuous group homomorphism from $\bigoplus \operatorname{Top}\left(S^{1} \times R^{m-1}\right)$ to $\operatorname{Top}_{0} M^{m}$ that induces an isomorphism on the homotopy groups $\pi_{i}$, for all indices $i \leq(m-4) / 3$. Furthermore, Mostow's rigidity theorem implies that Top $M$ is isomorphic to a semidirect product $\operatorname{Top}_{0} M \rtimes$ Out $\pi_{1} M$ and Out $\pi_{1} M$ is a finite (discrete) group.

Combining this result with the information about $\pi_{i} \operatorname{Top}\left(S^{1} \times R^{m-1}\right)$ contained in Lemma 10.15 (based on works of Waldhausen [63] and Hatcher and Wagoner [34]) yields the following calculation.

Corollary 10.16 (special case). If $M^{m}$ is a compact (connected) hyperbolic manifold whose dimension $m$ is greater than 10 , then $\pi_{i}$ Top $M \otimes Q=0$, provided $1 \leq i \leq(m-4) / 3$. Furthermore, $\pi_{0}$ Top $M$ is isomorphic to a semidirect product $\mathbf{Z}_{2}^{\infty} \rtimes$ Out $\pi_{1} M$, where $\mathbf{Z}_{2}^{\infty}$ denotes the countably infinite abelian group of exponent 2 .

We also obtain results about $\operatorname{Diff} M$, which is the group of all diffeomorphisms of $M$ with compact support. For example, [17, Theorem 4.5] combined with Theorem 9.5 and [25, Corollary 7.2] yields the following result.

Corollary 10.17. If $M^{m}$ is a compact (connected) hyperbolic manifold whose dimension $m$ is greater than 10 , then

$$
\pi_{s} \text { Diff } M^{m} \otimes Q=\left\{\begin{array}{l}
\bigoplus_{j=1}^{\infty} H_{(s+1)-4 j}\left(M^{m}, Q\right) \text { if } m \text { is odd }, \\
0, \quad \text { if } m \text { is even, }
\end{array}\right.
$$

provided $1 \leq s \leq(m-4) / 3$.

(The stable range in Corollaries 10.13, 10.16, and 10.17 depends on works of Igusa [37]. Works of Waldhausen [63], Hatcher [33], and Borel [5] also play important roles in these results.)

We also apply Theorem 9.5 to a third problem: homotoping a continuous map to a block bundle projection. Frank Quinn [57, 58] developed a general theory for attacking this problem, and Theorem 9.5 makes his theory effective when the range of the map is a compact hyperbolic manifold. This leads to our Theorem 10.7 (see $\S 10$ for its statement).

We now outline the proof of Corollary 10.3. We follow the same general outline as in [23] and [25]. The groups $K_{i}\left(\mathbf{Z} \pi_{1} M\right)$, for $i \leq 1$, were computed in [23], and the weak homotopy type of the stable pseudo-isotopy space of $M$ was analyzed in [25]. The proof has two main ingredients:

I. the foliated control theorem for structure spaces proved in [29], and

II. the dynamics of the geodesic flow (cf. [2]) together with other geometric ideas.

Our foliated control theorem [29] extends the unfoliated control results of [11] and [59]. Unlike our results in [23], [25], and [26], where we needed control theorems for foliations with only 1-dimensional leaves, the proof of Corollary 
10.3 (as well as our results in [24]) requires a foliated control theorem for foliations with higher dimensional leaves. ${ }^{2}$

The geometry of $M$ (i.e., that $M$ is a hyperbolic manifold) enters the proof by allowing us to equip the following constructions with extra structures and then enables us to analyze these structures. Let $S M$ denote the unit sphere bundle of $M, S \times S(M)$ the fiberwise product of $S M$ with itself (i.e., $S \times S(M)$ is the bundle associated to $S M$ whose fiber at $x$ consists of all ordered pairs of unit length vectors tangent to $M$ at $x$ ), and $C(M)$ the associated bundle whose fiber at $x$ consists of all unordered pairs of unit length vectors tangent to $M$ at $x$. (Except when confusion is possible, we will denote the total space of a bundle and the bundle itself by the same symbol.) Note that $C(M)$ is the orbit space of $S \times S(M)$ under the canonical fiberwise involution that transposes ordered pairs of vectors. Hence, its fiber is the space $S^{m-1} \times S^{m-1} / \sim$, where $\sim$ denotes the equivalence relation generated by $(x, y) \sim(y, x)$, where $x, y \in S^{m-1}$.

To abbreviate the many complications that arise in the proof of Corollary 10.3, we now assume that $M^{m}$ is orientable and odd dimensional, i.e., $m=$ $2 k+1$ for some positive integer $k>1$. The fiber of $C(M)$ is a stratified space having two strata, $\mathscr{A}$ and $\mathscr{D}$, consisting of agreeing and disagreeing pairs, respectively. Note that the bottom stratum $\mathscr{A}$ can be identified with the diagonal in $S^{2 k} \times S^{2 k}$ and hence is diffeomorphic to $S^{2 k}$, while $\mathscr{D}$ is a $4 k$ dimensional manifold. The fiber is also an orientable $\mathbf{Z}\left[\frac{1}{2}\right]$-homology manifold having index one. Let $C_{2 k+m}(M)$ and $C_{4 k+m}(M)$ denote the bundles associated to $S M$ with fibers $\mathscr{A}$ and $\mathscr{D}$, respectively. Then $C_{2 k+m}(M)$ and $C_{4 k+m}(M)$ are the two strata of a stratification of $C(M)$.

Using the fact that $M$ is a hyperbolic manifold, we can equip $C(M)$ with extra structures. Since $C(M)$ has an asymptotically defined flat structure, it has a foliation $\mathscr{F}$ that restricts to smooth foliations $\mathscr{F}_{2 k+m}$ and $\mathscr{F}_{4 k+m}$ for $C_{2 k+m}(M)$ and $C_{4 k+m}(M)$, respectively. We use the hyperbolicity of $M$ to "mark" the leaves of $\mathscr{F}_{2 k+m}$ and $\mathscr{F}_{4 k+m}$. Each leaf of the foliation of the bottom stratum is marked with an asymptotic vector field, while each leaf of the foliation of the top stratum is marked by a single geodesic. Both types of markings vary smoothly from leaf to leaf.

Consider a homotopy equivalence $h: N \rightarrow M$, where $N$ is a compact topological manifold. Let $C^{*}$ denote the pullback of the bundle $C(M)$ via $h$ and $h^{*}: C^{*} \rightarrow C(M)$ be the induced map on the total spaces of the bundles. We construct a compactly supported homotopy $h_{t}^{*}, t \in[0,1]$, with $h_{0}^{*}=h^{*}$ and such that $h_{1}^{*}$ is a homeomorphism over the bottom stratum $C_{2 k+m}(M)$ as well as over the complement of a tubular neighborhood for $C_{2 k+m}(M)$ in $C(M)$. We also arrange for $h_{1}^{*}$ to be "blocked up" over this tubular neighborhood. To get such a homotopy, we first flow $h^{*}$ over the bottom stratum in the direction

\footnotetext{
${ }^{2}$ The generalization of Corollary 10.3, in [66], uses only a foliated control theorem for foliations with one (and zero) dimensional leaves.
} 
of the asymptotic vector fields that mark the leaves of $\mathscr{F}_{2 k+m}$, thereby gaining 1-dimensional foliated control for $h^{*}$ over $C_{2 k+m}(M)$. Then we apply the structure space foliated control theorem from [26] or [29] for the 1-dimensional foliation of $C_{2 k+m}(M)$ consisting of flow lines (in fact, $C_{2 k+m}(M)$ can be identified with $S M$ and the flow with the geodesic flow) to construct the homotopy $h_{t}^{*}$ over the bottom stratum. To get $h_{t}^{*}$ over the top stratum (away from a tubular neighborhood for $C_{2 k+m}$ ), we use the markings by geodesics on the leaves of $\mathscr{F}_{4 k+m}$ together with the structure space foliated control theorem for arbitrary dimensional leaves but of a special geometric type, proved in [29]. Finally, $h_{t}^{*}$ is extended over the rest of the tubular neighborhood of the bottom stratum by using the fibered version of the foliated control theorem for foliations with 1-dimensional leaves, also proved in [29].

The homotopy equivalence $h$ is normally bordant to $\mathrm{id}_{M}$. (This was first proved in [18], although our proof of Corollary 10.3 is independent of [18].) This induces a stratified normal bordism from $h^{*}$ to $\mathrm{id}_{C(M)}$. If the fiber of $C(M)$ were a manifold, we could use standard surgery techniques to "desuspend" the homotopy $h_{t}^{*}$, i.e., to produce a homotopy $h_{t}: N \rightarrow M, t \in[0,1]$, such that $h_{0}=h$ and $h_{1}$ is a homeomorphism. (In this case, we would basically use the fact that crossing a normal map with the identity map on a simply connected index-one manifold does not change it surgery obstruction.) But the fiber of $C(M)$ is, in general, only an index-one $\mathbf{Z}\left[\frac{1}{2}\right]$-homology manifold. (Note that the fiber of $C(M)$ is the complex projective plane when $k=1$.)

To overcome this complication, we replace $M$ by its hyperbolic thickening $H M$ (see [23] and [25]), which is a special case of the warped product construction due to Bishop and O'Neill [4]. Recall that $H M$ is a complete hyperbolic manifold diffeomorphic to $M \times R$. (If $M$ is the orbit space of the action of a subgroup $\Gamma$ of $O(1, m)$ acting on $H^{m}$ by isometries, then $H M$ is the orbit space of the isomorphic subgroup $\hat{\Gamma}$ of $O(1, m+1)$ acting on $H^{m+1}$, where $\widehat{\Gamma}$ is the image of $\Gamma$ under the representation ${ }^{-}: O(1, m) \rightarrow O(1, m+1)$ defined in blocked matrix form by

$$
\widehat{A}=\left(\begin{array}{l|l}
A & 0 \\
\hline 0 & 1
\end{array}\right)
$$

where $A \in O(1, m)$.) The advantage of $H M$ over $M$ is that $S(H M)$ contains a canonical codimension-zero (closed) disc subbundle called its asymptotic northern hemisphere bundle. The boundary of (the total space of) this bundle is a codimension-one sphere subbundle called its asymptotic equator bundle. The construction of $C(M)$ is modified to produce a bundle $C^{\prime}=C^{\prime}(H M)$ over $H M$ whose fiber at $x \in H M$ consists of all unordered pairs of vectors $[u, v]$ tangent to $H M$ at $x$ but constrained so that both $u$ and $v$ lie in the asymptotic equator bundle. The fiber of $C^{\prime}$ is still an index-one $\mathbf{Z}\left[\frac{1}{2}\right]$-homology manifold (it is, in fact, homeomorphic to the fiber of $C(M)$ ), and $C^{\prime}$ is also stratified with two strata as before. The bottom stratum $C_{2 k+m+1}^{\prime}$ can be identified with the total space of the asymptotic equator bundle. Hence, $C_{2 k+m+1}^{\prime}$ is 
the boundary of the total space of the asymptotic northern hemisphere bundle. This is the reason for passing from $M$ to $H M$ because it allows us to isolate the "transferred surgery obstruction" over the top stratum and to show that it equals the original surgery obstruction.

Of course, $h$ is no longer a homotopy equivalence with range $M$, but rather a proper homotopy equivalence $h: N \rightarrow H M=M \times R$ that is a homeomorphism over the complement of $M \times[-1,1]$. It is still normally bordant to $\mathrm{id}_{H M}$, via a normal bordism with compact support. We can also construct a proper homotopy $h_{t}^{*}$, as above, so that it is constant over the complement of $M \times$ $[-2,2]$. Since $h_{1}^{*}$ is almost a homeomorphism, the surgery obstruction for the normal bordism between $h$ and $\mathrm{id}_{H M}$ is in the image of

$$
\Theta:\left[M^{m} \times I \times I \mathrm{rel} \partial ; G / \mathrm{Top}\right] \rightarrow L_{m+2}\left(\pi_{1} M\right)
$$

where $\Theta$ is part of the surgery exact sequence (cf. [64, 43]). Consequently, $\Theta$ is an epimorphism. In fact, it is an isomorphism. (It was first shown in [18] that $\Theta$ is a split monomorphism, but our arguments are independent of [18].) A fibered version of these arguments is used to show that $\Theta$ is an isomorphism when, in formula $(0.1), I \times I$ is replaced by $I^{n}$ and $L_{m+2}$ by $L_{m+n}$, provided both $n \geq 2$ and $m+n \geq 6$. (We showed, in [23], that $\mathrm{Wh}\left(\pi_{1} M \times A\right)=0$ for any finitely generated free abelian group $A$. Hence, the apparently different "torsion-decorated $L$-groups" all agree on $\pi_{1} M$.) It follows from the surgery exact sequence $[64,43]$ that $\mathscr{S}\left(M^{m} \times D^{n}\right.$ rel $\left.\partial\right)$ has cardinality one, provided both $m+n \geq 5$ and $n \geq 1$. But $\mathscr{S}(M)$ is in one-to-one correspondence with a subset of $\mathscr{S}\left(M \times D^{4}\right.$ rel $\left.\partial\right)$ by [43]. Hence, the cardinality of $\mathscr{S}(M)$ is also one. Because of the definition of $\mathscr{S}(M)$ (cf. $[64,43])$, this means that any homotopy equivalence $h: N \rightarrow M$ is homotopic to a homeomorphism, completing the sketch of the proof of Corollary 10.3.

Even though $M$ was assumed to be compact, we were forced to analyze the noncompact hyperbolic manifold $H M$ in proving Corollary 10.3. This is analogous to the corresponding situation in [23] where, in order to show that Wh $\pi_{1} M$ vanishes even when $M$ is a compact hyperbolic manifold, we were forced to consider $H M$. This considerably complicates our proof of Corollary 10.3 , as it did the proofs in our earlier papers [23] and [25], since metric control results for noncompact manifolds are more delicate. We develop, in $\S \S 2$ and 3 , metric lemmas needed to apply our basic control theorem for a structure set [29], enabling the construction of the homotopy $h_{t}^{*}$ posited above. The lemmas are, in fact, strong enough to allow us to prove our main result for arbitrary complete (connected) hyperbolic manifolds.

We now briefly describe the contents of each section. The basic properties of the bundle $C(M)$ are developed in $\S 1$, where $M$ is an arbitrary complete (connected) hyperbolic manifold. We show that $C\left(M^{m}\right)$ is a stratified space (with two strata) equipped with a foliation $\mathscr{F}$ by $m$-dimensional leaves that respects the stratification. This foliation comes from the fact that $C(M)$ has a natural flat bundle structure. Each leaf is equipped with a marking. The 
markings are of two types, depending on the stratum in which the leaf lies. Each leaf of the top stratum is marked by a geodesic, and the union of all these geodesics is a submanifold called the core of the top stratum. The top stratum is a fiber bundle over its core with contractible fiber.

We equip each stratum of $C(M)$ with a Riemannian metric in $\S 2$. These metrics are naturally determined by the hyperbolic metric on $M$. Each leaf $L$ of the foliation $\mathscr{F}$ is equipped with a primary and a secondary Riemannian metric. The primary metric is induced from the Riemannian metric on the stratum in which $L$ lies, and the secondary metric is determined by the fact that the bundle projection $C(M) \rightarrow M$ restricted to $L$ is an immersion. We also introduce a shelling of the top stratum by codimension-one submanifolds consisting of all points $x$ that are a constant distance $d$ from the marked geodesic as measured in the leaf containing $x$ using its secondary metric. The intersection of each of these shells with the leaves of $\mathscr{F}$ foliates the shell by new leaves that are locally isometrically equivalent to the Riemannian product $S^{m-2} \times R$, where $R$ is the flat line and $S^{m-2}$ is the round sphere of radius $r$ (which depends on $d$ ) with $\lim _{d \rightarrow+\infty} r=+\infty$.

We verify, in $\S 3$, the hypotheses of the foliated structure set control theorem [29] in the framework of this paper; i.e., we show that certain foliations are $(\beta, \sigma)$-flat on particular sets. These foliations include that of the shells mentioned above, the foliation of the total space of the asymptotic northern hemisphere subbundle by the flow lines of the geodesic flow, and the foliation of the core of the top stratum by its geodesic markings. In this verification, we make systematic use of Margulis' Lemma. We extend some of these results (usually in addenda) to arbitrary negatively curved complete (connected) locally symmetric spaces. The extended results are needed in the appendix. Since some of the constructions in $\S \S 1-3$ are used later in a more limited setting, we introduce specialized notation for them. $\S 3$ ends with a table explaining this specialized notation in terms of the general notation.

In sketching the proof of Corollary 10.3, we mention the need for a generalization of the result that surgery obstructions are unchanged when a normal map is multiplied with the identity map on $\mathbf{C} P^{2} . \S 4$ is devoted to formulating and proving the necessary generalization.

Also in the outline of the proof of Corollary 10.3, we posit the existence of a homotopy $h_{t}^{*}$. We formulate, in $\S 5$, a result, Theorem 5.5 , that can be used to produce $h_{t}^{*} . \S 6$ contains the formulation of two splitting theorems, Theorems 6.2 and 6.6, needed to prove Theorem 5.5. Theorem 6.6 is proved in [29], while the proof of Theorem 6.2 is a generalization of arguments in [21] and will appear in [30]. (Theorem 6.2 is not needed to prove Corollary 10.3.)

The asymptotic transfer is constructed in $\S 7$. It is an adaptation to homotopy equivalences of similar constructions, with the same name, made in [23] and [25] for $h$-cobordisms and psuedo-isotopies, respectively. The asymptotic transfer is an indispensible ingredient in the proof of Corollary 10.3. 
We complete the proof of Theorem 5.5 in $\S 8$ by applying results from earlier sections.

The main result of this paper, Theorem 9.5 , is proved in $\S 9$. It yields a calculation of the surgery obstruction groups ( $L$-groups) of the fundamental groups of complete (connected) hyperbolic manifolds of arbitrary dimension. The key ingredients in its proof are Theorems 4.3 and 5.5.

We give applications of Theorem 9.5 in $\S 10$. Many of these results were mentioned earlier in this introduction.

The appendix contains proofs of results, used in $\S 10$, about stable pseudoisotopy spaces. The result about $\mathscr{P}(M)$ was announced in [25] and generalizes [25, Theorem 6.0], while the result on $\mathscr{P}_{\text {Diff }}(M)$ is related to a theorem announced in [22].

This paper contains the proofs of the results announced in [27]. The possibility of a topological analogue to Mostow's rigidity theorem was suggested to one of the authors two decades ago by Robert H. Szczarba. Positive evidence for the truth of our topological rigidity result was provided by the earlier works of Mishchenko [49], Kasparov [41, 42], and Farrell and Hsiang [18, 20]. Also, Nicas and Stark [54, 55] and Nicas [53], previous to our work, calculated $L_{n}\left(\pi_{1} M^{3}\right) \otimes \mathbf{Z}\left[\frac{1}{2}\right]$ for certain interesting classes of nonsufficiently large hyperbolic 3-dimensional manifolds $M^{3}$.

\section{GeOMETRIC PRELIMINARIES}

Let $D^{n}$ denote the closed unit ball in Euclidean $n$-dimensional space $R^{n}$; i.e.,

$$
D^{n}=\left\{\left.x \in R^{n}|x \cdot x=| x\right|^{2} \leq 1\right\},
$$

where $x \cdot y$ and $|x|$ denote the Euclidean inner product and norm, respectively. Consider the open unit ball model (Poincare model) for hyperbolic $n$-space $H^{n}$; i.e., as a smooth manifold, $H^{n}$ is Int $D^{n}$ with Riemannian metric $h($, ) defined by

$$
h_{x}(X, Y)=\frac{4 X \cdot Y}{\left(1-|x|^{2}\right)^{2}},
$$

where $X$ and $Y$ are vectors tangent to $R^{n}$ at $x \in H^{n}$. Let Iso $H^{n}$ denote the group of all isometries of $H^{n}$; Iso $H^{n}$ is the Lie group $O_{0}(1, n)$. Recall that each isometry of $H^{n}$ extends to a unique diffeomorphism of $D^{n}$ giving a canonical differentiable action of the Lie group Iso $H^{n}$ on $D^{n}$. Since $S^{n-1}$ is the boundary of $D^{n}$, this action restricts to a canonical action of Iso $H^{n}$ on $S^{n-1}$. Let $\mathbf{Z}_{2}$ denote the cyclic group of order 2 (i.e., the additive group of the ring of integers $\bmod 2$ ) and $F_{n}$ denote the orbit space of the action of $\mathbf{Z}_{2}$ on $S^{n-1} \times S^{n-1}$, there the generator of $\mathbf{Z}_{2}$ acts on $S^{n-1} \times S^{n-1}$ via the diffeomorphism

$$
(x, y) \mapsto(y, x)
$$


for each $(x, y) \in S^{n-1} \times S^{n-1}$; i.e.,

$$
F_{n}=S^{n-1} \times \mathbf{z}_{2} S^{n-1} \text {. }
$$

Since the diagonal action of Iso $H^{n}$ on $S^{n-1} \times S^{n-1}$ commutes with the action of $\mathrm{Z}_{2}$ described in formula (1.3), we have a canonical induced action of Iso $H^{n}$ on $F_{n}$.

Note that $F_{n}$ is the set of all unordered pairs of points in $S^{n-1}$. While $F_{n}$ is not a manifold, it is a compact smoothly stratified space with two strata. The bottom stratum $\mathscr{A}$ consists of all unordered pairs where the two points in the pair agree; i.e., $\mathscr{A}$ is canonically homeomorphic to the diagonal in $S^{n-1} \times S^{n-1}$ (which is also the fixed point set of the action of $\mathrm{Z}_{2}$ on $S^{n-1} \times S^{n-1}$ ) via the quotient map. The top stratum $\mathscr{D}$ consists of all unordered pairs where the two points in the pair disagree. Note that the action of Iso $H^{n}$ on $F_{n}$ preserves this stratification.

Let $M$ be a (connected) complete (real) hyperbolic $n$-dimensional manifold. Then there exists a discrete torsion-free subgroup $\Gamma$ of Iso $H^{n}$ such that $M$ and the orbit space $H^{n} / \Gamma$ are isometrically equivalent. Hence, we identify $M$ with $H^{n} / \Gamma$ and $\pi_{1} M$ with $\Gamma$. Let $F(M)$ denote the orbit space of $H^{n} \times F_{n}$ under the diagonal action of $\Gamma$; i.e.,

$$
F(M)=H^{n} \times_{\Gamma} F_{n} .
$$

Note that $F(M)$ is the total space of the fiber over $M$ with fiber $F_{n}$ that is associated to the universal covering space of $M$. Thus, $F(M)$ is also a smoothly stratified space having bottom stratum $\mathscr{A}(M)$ and top stratum $\mathscr{D}(M)$, where

$$
\mathscr{A}(M)=H^{n} \times_{\Gamma} \mathscr{A} \text { and } \mathscr{D}(M)=H^{n} \times_{\Gamma} \mathscr{D} .
$$

Furthermore, $F(M)$ is equipped with a foliation $\mathscr{F}$ determined by the flat bundle structure (1.5). This foliation restricts, because of formula (1.6), to smooth foliations $\mathscr{F}_{a}$ and $\mathscr{F}_{d}$ for the strata $\mathscr{A}(M)$ and $\mathscr{D}(M)$, respectively. Let us explicitly describe the leaves of $\mathscr{F}$. For any point $u_{0}$ in $F(M)$, pick as point $\left(x_{0}, w_{0}\right)$ in $H^{n} \times F_{n}$ such that $u_{0}=p\left(x_{0}, w_{0}\right)$, where

$$
p: H^{n} \times F_{n} \rightarrow F(M)
$$

is the canonical quotient map. Then the leaf $L_{u_{0}}$ of $\mathscr{F}$ containing $u_{0}$ is the image of the level surface $H^{n} \times\left\{w_{0}\right\}$ (in $H^{n} \times F_{n}$ ) under $p$; i.e.,

$$
L_{u_{0}}=\left\{p\left(x, w_{0}\right) \mid x \in H^{n}\right\} .
$$

Note that $L_{u_{0}}$ is contained in $\mathscr{A}(M)$ or $\mathscr{D}(M)$ if and only if $u_{0}$ is an element of $\mathscr{A}(M)$ or $\mathscr{D}(M)$, respectively.

We now show that each leaf $L$ of $\mathscr{F}$ is equipped with an extra piece of geometric structure called a marking. These markings are of two types: if $L$ is in $\mathscr{D}(M)$, then it is marked by a geodesic "line" $l$ in $L$; if $L$ is in $\mathscr{A}(M)$, then it is marked by an "asymptotic" vector field $v($ ) on $L$. We proceed to 
describe these markings. Let $L=p\left(H^{n} \times w\right)$, where $w \in F_{n}$ and $w=\left[v_{0}, v_{1}\right]$ with each $v_{i} \in S^{n-1}$ and

$$
\begin{aligned}
& {\left[v_{0}, v_{1}\right] \text { denotes the image of the ordered pair }\left(v_{0}, v_{1}\right) \text { in }} \\
& S^{n-1} \times{ }_{\mathbf{Z}_{2}} S^{n-1}
\end{aligned}
$$

i.e., $\left[v_{0}, v_{1}\right]$ is the "unordered pair."

First consider the case where $v_{0} \neq v_{1}$; i.e., $L$ is a leaf of $\mathscr{F}_{d}$. Consider the unique circle (or straight line) in $R^{n}$ passing through $v_{0}$ and $v_{1}$ and meeting $S^{n-1}$ perpendicularly at these points. Let $\hat{l}$ be the intersection of this circle (line) with $H^{n}$. It is the unique geodesic line in $H^{n}$ that connects $v_{0}$ to $v_{1}$. Then the marking $l$ for $L$ is the image of $\hat{l} \times\{w\}$ under $p$; i.e.,

$$
l=p(\hat{l} \times\{w\}) .
$$

(Note that $l$ need not be homeomorphic to $R$ or even to the circle $S^{1}$.)

Next we consider the case where $v_{0}=v_{1}$; i.e., $L$ is a leaf of $\mathscr{F}_{a}$. We mark $L$ with the "asymptotic" vector field $v($ ) (with domain $L$ ) whose value at $p(x, w)$ (where $x \in H^{n}$ ) is determined by the following procedure. First construct the unique circle (or straight line) in $R^{n}$ that passes through $x$ and $v_{0}$ and intersects $S^{n-1}$ perpendicularly. Then

$$
v(p(x, w))=d p\left(u_{x}\right),
$$

where $u_{x}$ is the vector tangent to $H^{n} \times\{w\}$ such that $d q\left(u_{x}\right)$ is the unit length vector in $H^{n}$ tangent to the constructed circle (line) at $x$ and that points into that arc of this circle (line) that is contained in $D^{n}$ and connects $x$ to $v_{0}$. Here

$$
q: H^{n} \times F_{n} \rightarrow H^{n}
$$

denotes projection onto the first factor.

We now look at these markings from a different point of view. First we identify the lower stratum $\mathscr{A}(M)$ with $S M$, i.e., to the total space of the tangent unit sphere bundle of $M$. We do this by constructing a $\Gamma$-equivariant diffeomorphism $f$ from $S H^{n}$ to $H^{n} \times \mathscr{A}$. Let $v$ be a unit length vector tangent to $H^{n}$ at a point $x$ in $H^{n}$. Then

$$
f(v)=(x, w), \quad \text { where } w=[u, u],
$$

and $u$ is defined as follows. Construct the unique circle (or straight line) in $R^{n}$ having $v$ as a tangent vector and intersecting $S^{n-1}$ perpendicularly. Then $u$ is the point of intersection of this circle (line) with $S^{n-1}$ such that $v$ points into the arc of the circle (line) connecting $x$ to $u$. This is clearly a $\Gamma$-equivariant diffeomorphism. Hence, it induces the posited identification. Under this identification, $\mathscr{F}_{a}$ is the weakly unstable foliation of the geodesic flow $g^{t}$ on $S M$. The tangent vectors to the flow lines of $g^{t}$ form a vector field on $S M$, which, restricted to each leaf $L$ in $\mathscr{F}_{a}$, is the "asymptotic" vector field described above that marks $L$. 
Next we identify $P M$ with a submanifold of $\mathscr{D}(M)$. Here $P M$ denotes the total space of the real projective $(n-1)$-space bundle associated to the tangent bundle $T M$; i.e., $P M$ is the orbit space of the free action of $\mathrm{Z}_{2}$ on $S M$, where the generator of $\mathrm{Z}_{2}$ acts via $v \mapsto-v$ for each vector $v$ in $S M$. Our identification is determined by the following $\Gamma$-equivalent embedding $g$ of $P H^{n}$ into $H^{n} \times \mathscr{D}$. Let $[v]$ in $P H^{n}$ be the equivalence class of a unit length vector $v$ tangent to $H^{n}$ at a point $x$ in $H^{n}$. Then

$$
g([v])=(x, w), \quad \text { where } w=\left[u_{1}, u_{2}\right],
$$

and the unordered pair $\left[u_{1}, u_{2}\right]$ is defined as follows. Construct the unique circle (or straight line) in $R^{n}$ having $v$ as a tangent vector and intersecting $S^{n-1}$ perpendicularly. This circle (line) intersects $S^{n-1}$ in the pair of points $\left[u_{1}, u_{2}\right]$.

Furthermore, $\mathscr{D}(M)$ is the total space of a smooth fiber bundle with base space $P M$ and fiber $H^{n-1}$ (its structure group has a natural reduction to $O(n-1)$ because of the " 0 -section" $P M)$ via a smooth retraction map

$$
p_{d}: \mathscr{D}(M) \rightarrow P M,
$$

which we get from a $\Gamma$-equivariant map $\bar{p}_{d}: \mathscr{D}\left(H^{n}\right) \rightarrow P H^{n}$ constructed as follows. Let $\left(x,\left[u_{1}, u_{2}\right]\right)$ be a point in $\mathscr{D} H^{n}$. First construct the unique circle (or straight line) in $R^{n}$ that intersects $S^{n-1}$ perpendicularly in $\left\{u_{1}, u_{1}\right\}$ (recall $u_{1} \neq u_{2}$ ). Let $\hat{l}$ be the arc of this circle (line) contained in $H^{n}$ (i.e., its intersection with $\left.H^{n}\right)$. Next construct an $(n-1)$-sphere (or $(n-1)$-plane) $S$ in $R^{n}$ that intersects both $S^{n-1}$ and $\hat{l}$ perpendicularly and contains $x$ (if $x \notin \hat{l}, S$ is unique). Then

$$
\bar{p}_{d}\left(x,\left[u_{1}, u_{2}\right]\right)=(y,[v]) \text {, }
$$

where $y$ is the point at which $\hat{l}$ and $S$ intersect and $v$ is a unit length vector tangent to $\hat{l}$ at $y$. The following fact is easily verified. In its statement, $q: S M \rightarrow P M$ denotes the canonical covering projection.

Lemma 1.1. If $L$ is a leaf of the foliation $\mathscr{F}_{d}$, then $l=L \cap P M$ is its marking and $L=p_{d}^{-1}(l)$, where $p_{d}: \mathscr{D}(M) \rightarrow P M$ is the fiber bundle in (1.15). Furthermore, let $v$ be any vector in $S M$ such that $q(v) \in L$ and let $\bar{l}$ denote its orbit under the geodesic flow; i.e., $\bar{l}=\left\{g^{t} v \mid t \in R\right\}$. Then $q$ maps $\bar{l}$ homeomorphically onto the marking $l$.

We also have the following result.

Lemma 1.2. $P M$ is a closed subset of $F(M)$.

Proof. Since $p_{d}: \mathscr{D}(M) \rightarrow P M$ is a retraction, $P M$ is a closed subset of $\mathscr{D}(M)$. Consequently, it is sufficient to show that a sequence of points in $P M$ cannot converge to a point in $S M$. In fact, since $P H^{n}$ and $S H^{n}$ are both $\Gamma$-invariant, it suffices to show that a sequence of points $\left(x_{n}, w_{n}\right)$ in $P H^{n}$ cannot converge to a point $(x, w)$ in $S H^{n}$. On the contrary, suppose this happens. 
Then $x_{n}$ converges to $x$ and $w_{n}$ converges to $w$, since $F\left(H^{n}\right)=H^{n} \times F_{n}$. Let $w_{n}=\left[u_{n}, v_{n}\right]$ and $w=[v, v]$ (recall that $\left.S H^{n}=\mathscr{A}\left(H^{n}\right)=H^{n} \times \mathscr{A}\right)$. Then both $u_{n}$ and $v_{n}$ converge to $v$. Since $\left(x_{n}, w_{n}\right) \in P H^{n}, x_{n} \in \hat{l}_{n}$, where $\hat{l}_{n}$ is the intersection of $H^{n}$ with the circle (or straight line) in $R^{n}$ that intersects $S^{n-1}$ perpendicularly in $\left\{u_{n}, v_{n}\right\}$. Since both $u_{n}$ and $v_{n}$ converge to $v$, the length of $\hat{l}_{n}$ converges to 0 and hence $\left|x_{n}\right|$ converges to 1 . On the other hand, $\left|x_{n}\right|$ converges to $|x|$, which is less than 1 , since $x \in H^{n}$. This contradiction completes the proof of Lemma 1.2.

We have thus far expressed our constructions using, for the most part, the terminology of synthetic geometry. But we will also need its differential geometric formulation. For this we recall the following dictionary. The totally geodesic $k$-dimensional submanifolds of $H^{n}$ are the intersections $P \cap H^{n}$, where $P$ is a $k$-sphere (or $k$-plane) in $R^{n}$ that intersects $S^{n-1}$ perpendicularly. In particular, the geodesic lines in $H^{n}$ are the intersections $P \cap H^{n}$ of the above type, where $P$ is a circle (or straight line). The ideal boundary of $H^{n}$ is $S^{n-1}$. Recall that a point on the ideal boundary is an equivalence class of geodesic rays in $H^{n}$, where two rays are equivalent if they are asymptotic, i.e., if they are a finite Hausdorff distance apart. In fact, if the geodesic ray $r$ represents a point of the ideal boundary of $H^{n}$, then the intersection of the closure of $r$ with $S^{n-1}$ is the corresponding point on $S^{n-1}$.

Next we describe $F(M)$ in terms of the unit tangent bundle $S M$ of $M$. To do this, let $S_{2}(M)$ denote the orbit space of the diagonal action of $\Gamma$ on $H^{n} \times\left(S^{n-1} \times S^{n-1}\right)$; i.e.,

$$
S_{2}(M)=H^{n} \times_{\Gamma}\left(S^{n-1} \times S^{n-1}\right) .
$$

(Recall that $\Gamma$ also acts diagonally on $S^{n-1} \times S^{n-1}$.) Since the natural action of $\mathbf{Z}_{2}$ on $S^{n-1} \times S^{n-1}$ described in (1.3) commutes with the diagonal action of $\Gamma$ on $S^{n-1} \times S^{n-1}$, we have an induced action of $\mathbf{Z}_{2}$ on $S_{2}(M)$ whose orbit space is $F(M)$. On the other hand, denote the space consisting of all pairs of unit length vectors tangent to $M$ at a common point by $S_{2} M$; i.e.,

$$
S_{2} M=\{u, v \in S M \mid q(u)=q(v)\} \text {, }
$$

where $q: S M \rightarrow M$ is the bundle projection. Note that $S_{2} M$ is the total space of a smooth fiber bundle with fiber diffeomorphic to $S^{n-1} \times S^{n-1}$ and bundle projection denoted by $q_{2}$. The cyclic group $\mathrm{Z}_{2}$ acts on the total space of this bundle as a fiber preserving group of diffeomorphisms. Denote the orbit spaced of this actions by $F M$. It is the total space of a fiber bundle over $M$ with fiber $F_{n}$ and is smoothly stratified with top and bottom strata denoted by $\mathscr{D} M$ and $\mathscr{A} M$, respectively, where $\mathscr{A} M$ is the fixed point set of the action of $\mathrm{Z}_{2}$ and $\mathscr{D} M$ is the complement of $\mathscr{A} M$ in $F M$. The points in $F M$ are the unordered pairs $\left[v_{1}, v_{2}\right]$ of vectors $v_{1}, v_{2}$ in $S M$ with $q\left(v_{1}\right)=q\left(v_{2}\right)$. We, of course, identify $S M$ with $\mathscr{A} M$ via the correspondence

$$
v \mapsto[v, v],
$$


where $v \in S M$. We canonically identify $S_{2} M, F M, \mathscr{A} M$, and $\mathscr{D} M$ (together with their bundle projections to $M$ ) with the spaces $S_{2}(M), F(M)$, $\mathscr{A}(\boldsymbol{M})$, and $\mathscr{D}(\boldsymbol{M})$, respectively, by constructing a $\Gamma \times \mathbf{Z}_{2}$-equivariant diffeomorphism $\hat{f}: S_{2} H^{n} \rightarrow H^{n} \times\left(S^{n-1} \times S^{n-1}\right)$. Let $v_{1}, v_{2}$ be a pair of unit length vectors tangent to $H^{n}$ at a point $x$ in $H^{n}$. Then

$$
\hat{f}\left(v_{1}, v_{2}\right)=\left(x ; u_{1}, u_{2}\right) \text {, }
$$

where $u_{i} \quad(i=1,2)$ is the point in $S^{n-1}$ represented by the geodesic ray $\left\{\gamma_{v_{i}}(t) \mid t \geq 0\right\}$. We use here the notation of formula (3.3) in [23]; namely, for each vector $v$ in $S M, \gamma_{v}$ denotes the geodesic in $M$ such that

$$
\dot{\gamma}_{v}(0)=v \text {. }
$$

Note that our identification of $S M$ with $\mathscr{A} M$ and $\mathscr{A}(M)$, respectively, are consistent under the above identification of $F M$ to $F(M)$. Also, $\hat{f}$ induces the following embedding of $P M$ into $\mathscr{D} M$; namely,

$$
[v] \mapsto[v,-v],
$$

where $v \in S M$. Furthermore, the fiber bundle map $p_{d}$ of (1.15) corresponds to the following map $\mathscr{D} M \rightarrow P M$ also denoted by $p_{d}$. It is induced by the $\Gamma$-equivalent map $\hat{p}_{d}: \mathscr{D} H^{n} \rightarrow P H^{n}$ given by

$$
\hat{p}_{d}\left[v_{1}, v_{2}\right]=[v] \text {, }
$$

where $v$ in $S H^{n}$ is uniquely defined by the following properties:

(i) the geodesic rays $\left\{\gamma_{v}(t) \mid t \geq 0\right\}$ and $\left\{\gamma_{v_{1}}(t) \mid t \geq 0\right\}$ are asymptotic as are the rays $\left\{\gamma_{v}(t) \mid t \leq 0\right\}$ and $\left\{\gamma_{v_{2}}(t) \mid\right.$ $t \geq 0\}$

(ii) $q(v)$ equals the orthogonal projection of $q\left(v_{1}\right)=q\left(v_{2}\right)$ onto the geodesic line $\left\{\gamma_{v}(t) \mid t \in R\right\}$.

Under our identification, the leaves of the foliation $\mathscr{F}$ of $F M$ are the graphs of the asymptotic vector fields. To be precise, let the unordered pair $[u, v]$ be a point in $F H^{n}=H^{n} \times F_{n}$ and $p: F H^{n} \rightarrow F M$ denote the covering projection. Then the leaf $L$ of $\mathscr{F}_{d}$ containing $p[u, v]$ is described by

$$
L=\left\{p[u(x), v(x)] \mid x \in H^{n}\right\},
$$

where $u()$ and $v()$ are the vector fields on $H^{n}$ consisting of all vectors asymptotic to $u$ and $v$, respectively. (See [23, p. 547] for the precise definition of the asymptotic vector field $v()$.) If $u \neq v$, let $w$ be a vector in $S H^{n}$ such that $\dot{\gamma}_{w}(0)$ and $-\dot{\gamma}_{w}(0)$ are asymptotic to $u$ and $v$, respectively. Then $L$ is marked by

$$
\left\{p\left[\dot{\gamma}_{w}(t),-\dot{\gamma}_{w}(t)\right] \mid t \in R\right\} .
$$

On the other hand, if $u=v$, then the "asymptotic" vector field that marks $L$ is the image of the asymptotic vector field $v()$, defined on $H^{n}$, under the 
derivative of the composite of the canonical identification of $H^{n}$ to $H^{n} \times[v, v]$ and the covering projection $p: H^{n} \times F_{n} \rightarrow F M$.

\section{METRIC PROPERTIES OF FM}

The Riemannian metric $h($,$) on M$ (determined by formula (1.2)) induces canonically a Riemannian metric $\hat{h}($,$) on S_{2} M$ that generalizes the canonically induced Riemannian metric $\bar{h}($,$) on S M$ described in [23, p. 547]. Let $w_{1}(t), w_{2}(t):(-\varepsilon, \varepsilon) \rightarrow S_{2} M$ be $C^{1}$ curves tangent at $t=0$ to vectors $\eta_{1}$, $\eta_{2}$ in $T_{w} S_{2} M$, where $w=w_{1}(0)=w_{2}(0)$. Note that $w_{i}(t)=\left(u_{i}(t), v_{i}(t)\right)$, where (for $i=1$ or 2 )

$$
u_{i}(t), v_{i}(t):(-\varepsilon, \varepsilon) \rightarrow S M
$$

are unit length vector fields along a $C^{1}$ curve $\alpha_{i}(t)$ in $M$, where $\alpha_{i}(t)=$ $q_{2}\left(w_{i}(t)\right)$ and $\alpha_{1}(0)=\alpha_{2}(0)=q_{2}(w)$. (Recall that $q_{2}: S_{2} M \rightarrow M$ is the bundle projection.) Let $U_{i}, V_{i}(i=1,2)$ denote the covariant derivatives of $u_{i}(t), v_{i}(t)$, respectively, along $\alpha_{i}(t)$ when $t=0$. Then

$$
\hat{h}_{w}\left(\eta_{1}, \eta_{2}\right)=h_{x}\left(\dot{\alpha}_{1}(0), \dot{\alpha}_{2}(0)\right)+\frac{1}{2} h_{x}\left(U_{1}, U_{2}\right)+\frac{1}{2} h_{x}\left(V_{1}, V_{2}\right),
$$

where $x=\alpha_{1}(0)=q_{2}(w)$. Since $\mathbf{Z}_{2}$ acts on $S_{2} M$ via isometries, $\hat{h}$ induces a Riemannian metric on each stratum of $F M$. The Riemannian metric of $\mathscr{D} M$ is also denoted by $\hat{h}$ while the Riemannian metric on $\mathscr{A} M=S M$ is $\bar{h}$; i.e., it is the Riemannian metric induced on $S M$ from $h$ as described in [23, p. 547].

Lemma 2.1. The following inequalities hold for any vector $\eta$ tangent to a leaf of the foliation $\mathscr{F}$ of $F M$.

$$
2 h(d q(\eta), d q(\eta)) \geq \hat{h}(\eta, \eta) \geq h(d q(\eta), d q(\eta)),
$$

where $q: \mathscr{D} M \rightarrow M$ denotes the bundle projection.

Proof. This result is a consequence of [23, Lemma 3.1]. It suffices to consider the case where $M$ is $H^{n}$. Then there exist vectors $u, v$ in $S H^{n}$ such that $\eta$ is the tangent vector, at $t=0$, to a curve $w(t)=[u(\alpha(t)), v(\alpha(t))]$, where $\alpha(t)$ is a smooth curve in $H^{n}$ and $u(), v()$ denote the vector fields asymptotic to $u, v$, respectively. (See [23, p. 547].) Since $d q(\eta)=\dot{\alpha}(0)$, our result follows from (2.1) and the estimate on the length of the covariant derivative of an asymptotic vector field given in [23, Lemma 3.1].

Note that each leaf $L$ of $\mathscr{F}$ has two natural Riemannian metrics denoted by $\hat{h}$ and $h$. The primary Riemannian metric $\hat{h}$ is the restriction of the Riemannian metric $\hat{h}$ on $F M$ to $L$ while the secondary Riemannian metric $h$ is induced from the Riemannian metric $h$ on $M$ via the covering projection $q: L \rightarrow M$. (Here $q$ denotes the restriction to $L$ of the bundle projection $q: F M \rightarrow M$.) With this terminology, Lemma 2.1 has the following obvious reformulation. 
Corollary 2.2. For any leaf $L$ of $\mathscr{F}$ and any vector $\eta$ tangent to $L$, we have that

$$
2 h(\eta, \eta) \geq \hat{h}(\eta, \eta) \geq h(\eta, \eta) .
$$

We next discuss some elementary properties of hyperbolic space that are needed to analyze the metric properties of the fiber bundle $p_{d}: \mathscr{D} M \rightarrow P M$. Fix a geodesic line $l$ in $H^{n}$, and for each positive real number $r$, let $C_{r}$ denote the shell in $H^{n}$ of radius $r$ and axis $l$; i.e.,

$$
C_{r}=\{x \mid \text { distance from } x \text { to } l \text { is } r\} .
$$

Given two distinct positive real numbers $r_{0}$ and $r_{1}$, there is a canonical diffeomorphism $f: C_{r_{1}} \rightarrow C_{r_{0}}$ defined by

$$
\begin{aligned}
& f(x)=y \text { provided the geodesic line containing both } x \text { and } y \\
& \text { meets } l \text { perpendicularly at a point which is not on the segment } \\
& \text { between } x \text { and } y \text {. }
\end{aligned}
$$

We have the following two results whose proofs are routine exercises in hyperbolic geometry.

Lemma 2.3. Put on the shell $C_{r}$ the Riemannian metric inherited by being a submanifold of $H^{n}$. Then $C_{r}$ is a complete Riemannian manifold that is isometrically equivalent to the Riemannian product $S \times R$, where $S$ is the $(n-2)$-sphere of constant sectional curvature equal to $(\sinh r)^{-2}$ and $R$ is the flat line.

Lemma 2.4. Given a positive real number $\alpha$, there exists a positive real number $\beta$ such that $f: C_{r_{1}} \rightarrow C_{r_{0}}$ is a strong $\beta$-Lipshitz map; i.e., for each vector $\eta$ tangent to $C_{r_{1}}$, we have that

$$
|\eta| \geq|d f(\eta)| \geq \beta|\eta|
$$

provided $\alpha \leq r_{0}<r_{1} \leq r_{0}+\alpha$.

We proceed to associate to the bundle $p_{d}: \mathscr{D} M \rightarrow P M$ and each positive real number $r$ a subbundle

$$
p_{d}: \mathscr{D}_{r} M \rightarrow P M
$$

whose fibers are diffeomorphic to $S^{n-1}$. The total space $\mathscr{D}_{r} M$ of this bundle is defined as follows.

Definition 2.5. The total space $\mathscr{D}_{r} M$ consists of all points $x$ in $\mathscr{D} M$ such that the distance in $L$ between $x$ and $p_{d}(x)$ is $r$, where $L$ is the leaf of $\mathscr{F}_{d} M$ containing $x$ and the distance is measured in terms of the secondary Riemannian metric $h$ on the leaf $L$.

Note that Iso $H^{n}$ acts transitively on $\mathscr{D}_{r} H^{n}$ and that $p_{d}: \mathscr{D} H^{n} \rightarrow P H^{n}$ is Iso $H^{n}$-equivariant. These facts together with the arguments used in proving Lemma 1.2 yield the following result. 
Lemma 2.6. The space $\mathscr{D}_{r} M$ is a smooth submanifold of $\mathscr{D} M$ and a closed subset of FM. Furthermore, $p_{d}: \mathscr{D}_{r} M \rightarrow P M$ is a smooth fiber bundle whose fiber is diffeomorphic to $S^{n-1}$.

Definition 2.7. For each positive real number $r$, let $\widehat{\mathscr{D}}_{r} M$ denote the subspace of $\mathscr{D} M$ consisting of all points $x$ such that the distance in $L$ between $x$ and $p_{d}(x)$ is $r$, where $L$ is the leaf in $\mathscr{F}_{d} M$ containing $x$ and the distance is measured in terms of the primary Riemannian metric $\hat{h}$ on the leaf $L$.

Lemma 2.8. There is a continuous and strictly increasing function $r \rightarrow \hat{r}$ (whose domain is the positive real numbers) such that

(i) $\mathscr{D}_{\hat{r}} M=\widehat{\mathscr{D}}_{r} M$,

(ii) $r / \sqrt{2} \leq \hat{r} \leq r$, and

(iii) $0 \leq(\widehat{r+s})-\hat{r} \leq s$

for all $r, s>0$.

Proof. It suffices to consider the case where $M=H^{n}$. With respect to the Riemannian metric $\hat{h}$ on $\mathscr{D} H^{n}$, Iso $H^{n}$ acts on $\mathscr{D} H^{n}$ as a group of isometries each of which respect the foliation $\mathscr{F}_{d}$. That is, if $L$ is a leaf of $\mathscr{F}_{d}$ and $\gamma \in$ Iso $H^{n}$, then $\gamma(L)$ is also a leaf of $\mathscr{F}_{d}$, and $\gamma$ restricted to $L$ is an isometry of $L$ to $\gamma(L)$ if both leaves are given their primary Riemannian metric. (This is also true if both leaves are given their secondary Riemannian metrics.) Consequently, Iso $H^{n}$ leaves $\widehat{\mathscr{D}}_{r} H^{n}$ invariant since $P H^{n}$ is an Iso $H^{n}$-invariant set. Pick a point $x$ in $\widehat{\mathscr{D}}_{r} H^{n}$, and let $\hat{r}$ be the distance of $x$ to $p_{d}(x)$. Then $x \in \mathscr{D}_{\hat{r}} H^{n}$. Since (Iso $\left.H^{n}\right) x=\mathscr{D}_{\hat{r}} H^{n}$ (see the remark following Definition 2.5), $\mathscr{D}_{\hat{r}} H^{n}$ is a subset of $\widehat{\mathscr{D}}_{r} H^{n}$. Continuing this line of reasoning, one easily sees that $\mathscr{D}_{\hat{r}} H^{n}$ fills up all of $\widehat{\mathscr{D}}_{r} H^{n}$; i.e.,

$$
\mathscr{D}_{\hat{r}} H^{n}=\widehat{\mathscr{D}}_{r} H^{n} \text {. }
$$

From Corollary 2.2, we deduce the truth of (ii) and (iii) above. The fact that the function $\hat{r}$ is both increasing and continuous is a consequence of (iii).

The intersection of the leaves of the foliation $\mathscr{F}_{d}$ with the submanifold $\widehat{\mathscr{D}}_{r} M$ induces a foliation $\widehat{\mathscr{G}}_{r}$ of $\widehat{\mathscr{D}}_{r} M$. Also, $\widehat{\mathscr{D}}_{r} M$ inherits a Riemannian metric from the Riemannian metric $\hat{h}$ on $\mathscr{D} M$. Denote this Riemannian metric by $\hat{h}$ also.

Lemma 2.9. Let $L$ be a leaf of the foliation $\hat{\mathscr{G}}_{r}$ of $\widehat{\mathscr{D}}_{r} M$. Equip $L$ with the leaf topology and the Riemannian metric induced from $\widehat{\mathscr{D}}_{r} M$. Then there exists a regular covering space $p_{L}: C_{\bar{r}} \rightarrow L$, where $C_{\bar{r}}$ is the codimension-one shell in $H^{n}$ defined by formula (2.2) such that $p_{L}$ is a local isometry. Furthermore, the following inequalities are true:

(i) $\sqrt{2} r \geq \bar{r} \geq r / \sqrt{2}$ and

(ii) $\sqrt{2} \hat{r} \geq \bar{r} \geq \hat{r}$,

where $\hat{r}$ is the function posited in Lemma 2.8 . 
(Recall that Lemma 2.3 shows that $C_{\bar{r}}$ is isometrically equivalent to the Riemannian product of the flat line and the round sphere of radius $\sinh \bar{r}$.)

Proof. We first note that (i) is a formal consequence of (ii) because of Lemma 2.8. Let $p: \mathscr{D} H^{n} \rightarrow \mathscr{D} M$ denote the covering projection and $\mathscr{L}$ be the leaf of $\mathscr{F}_{d}$ containing $L$. Then there exists a leaf $\mathscr{L}$ of the foliation $\mathscr{F}$ of $\mathscr{D} H^{n}$ such that $p(\overline{\mathscr{L}})=\mathscr{L}$ and

$$
\bar{p}_{\mathscr{L}}: \overline{\mathscr{L}} \rightarrow \mathscr{L}
$$

is a regular covering space (with $\mathscr{L}$ and $\overline{\mathscr{L}}$ given the leaf topology), where $\bar{p}_{\mathscr{L}}$ denotes the restriction of $p$ to $\overline{\mathscr{L}}$. Furthermore, $\bar{p}_{\mathscr{L}}$ is a local isometry when the two leaves are both equipped with either their primary or secondary Riemannian metrics. Arguing in this manner, we see that to prove Lemma 2.9 it suffices to consider the case where $M=H^{n}$.

Let $\bar{l}$ be the geodesic line that marks $\mathscr{L}=\overline{\mathscr{L}}$. In terms of the secondary Riemannian metric $h$ on $\mathscr{L}$, the pair $(\mathscr{L}, \bar{l})$ is isometrically equivalent to the pair $\left(H^{n}, l\right)$, where $l$ is a geodesic line in $H^{n}$ via an isometry

$$
f: \mathscr{L} \rightarrow H^{n} .
$$

Since $L=\mathscr{L} \cap \widehat{\mathscr{D}}_{r} H^{n}$, we have by Lemma 2.8 that

$$
L=\mathscr{L} \cap \mathscr{D}_{\hat{r}} H^{n} .
$$

The leaf $L$ also has two natural Riemannian metrics. We denote the primary one by $\hat{h}$ and the secondary one by $h$. They are induced from the primary and secondary Riemannian metrics $\hat{h}$ and $h$ on $\mathscr{L}$, respectively. In terms of $h, f$ induces an isometry from $L$ to $C_{\hat{r}}$. Let $G^{L}$ denote the subgroup of Iso $H^{n}$ that leaves $L$ invariant. Then $G^{L}$ acts on $L$ via isometries in both the primary and secondary Riemannian metrics on $L$. Using the strong transitivity properties of this action on $L$, we can explicitly compare $\hat{h}$ with $h$. In this way, we can deduce that $L$ is isometrically equivalent to $C_{\bar{r}}$, where $\bar{r}$ satisfies the inequalities posited in (ii). The details are left to the reader.

\section{LEAVES OF SMALL INJECTIVITY RADIUS}

Recall that each shell $\widehat{\mathscr{D}}_{r} M$ is equipped with the foliation $\hat{\mathscr{G}}_{r}$ obtained by intersecting the leaves of $\mathscr{F}_{d}$ with $\widehat{\mathscr{D}}_{r} M$. Furthermore, the core $P M$ of $\mathscr{D} M$ also inherits a 1-dimensional foliation denoted by $\mathscr{G}$, in the same way; i.e., the leaves of $\mathscr{G}$ are the intersections of the leaves of $\mathscr{F}_{d}$ with $P M$. The following result, in which $p: S M \rightarrow P M$ is the covering projection, is an immediate consequence of the discussion in $\S \S 1$ and 2, namely, of Lemma 1.1, Corollary 2.2, Lemma 2.3, and Lemma 2.9. The details of its proof are left to the reader. 
Lemma 3.1. Let $p(v)$ be a point in $P M$, where $v \in S M$, and let $\mathscr{L}$ be the leaf of $\mathscr{F}_{d}$ containing $p(v)$. Then the "geodesic line" $l$ that marks $\mathscr{L}$ is the leaf of $\mathscr{G}$ containing $p(v)$. Also, $l=p(\bar{l})$, where $\bar{l}=\left\{g^{t} v \mid t \in R\right\}$; i.e., $\bar{l}$ is the orbit of $v$ under the geodesic flow $g^{t}$. Furthermore, let $w$ be a point in $\widehat{\mathscr{D}}_{r} M$ such that $p_{d}(w)=p(v)$ and $L$ the leaf of $\widehat{\mathscr{G}}_{r}$ containing $w$. Then $L=p^{-1}(l)$. Also, we have the following dichotomy in which $p_{L}: C_{\bar{r}} \rightarrow L$ is the regular covering space posited in Lemma 2.9:

(i) if $l$ is not compact, then $p_{L}$ is an isometry;

(ii) if $l$ is compact, then $L$ is also compact and the radius of injectivity of $L$ is greater than the minimum of $2 \sinh \bar{r}$ and one quarter of the arc length of the simple closed geodesic $\bar{l}$.

We now analyze the foliation $\overline{\mathscr{G}}$ of $S M$ defined by (3.1) the leaves of $\bar{G}$ are the orbits of the geodesic flow $g^{t}$ on $S M$.

But first we recall, and then use, the following notation from [3, §8]. Let $x \in M$ and $y \in H^{n}$ be points such that $p(y)=x$, where $p: H^{n} \rightarrow H^{n} / \Gamma=M$ denotes the covering projection. Furthermore, let $\gamma \in \Gamma$.

(i) $\operatorname{Inj} \operatorname{Rad}(x)$ is the injectivity radius of $M$ at $x$;

(ii) $d_{\gamma}(y)$ is the distance between $y$ and $\gamma(y)$;

(iii) $d_{\Gamma}(y)=\min _{\gamma \in \Gamma-\text { id }} d_{\gamma}(y)=2 \operatorname{Inj} \operatorname{Rad}(x)$;

(iv) $\mu$ denotes the positive number $\mu(n)$ posited in Margulis' Lemma applied to $H^{n}$ (cf. [3, Theorem, p. 101]).

Given a positive number $\mu_{0}<\frac{1}{2} \mu$, define sets $M_{\mu_{0}}$ and $(S M)_{\mu_{0}}$ as follows:

(i) $M_{\mu_{0}}=\left\{x \in M \mid \operatorname{Inj} \operatorname{Rad}(x)<\mu_{0}\right\}$,

(ii) $(S M)_{\mu_{0}}=q^{-1}\left(M_{\mu_{0}}\right)$,

where $q: S M \rightarrow M$ denotes the bundle projection. If $\alpha$ is a piecewise smooth path in a Riemannian manifold, then

(i) $|\alpha|$ denotes the arclength of $\alpha$, and

(ii) image $\alpha$ denotes the image of $\alpha$.

An elementary packing argument yields the next result, which is used to prove Lemma 3.1.2.

Remark 3.1.1. To each positive integer $n$ is associated a positive constant $C_{n}$ such that the following statement is true. If $x_{1}, x_{2}, \ldots, x_{i}$ is any finite sequence of points on the $(n-1)$-sphere of radius 1 , then there exist distinct indices $s, t \in\{1,2, \ldots, i\}$ such that

$$
d\left(x_{s}, x_{t}\right) \leq C_{n}(i)^{-1 / n}
$$


Lemma 3.1.2. To each positive integer $n$ is associated a positive number $K_{n}$ such that the following statement is true. Let $\alpha$ be any piecewise smooth loop in $M$ (with $|\alpha| \leq 1$ ) and $v \in S_{\alpha(0)} M$ be any vector. Then there exists a piecewise smooth loop $\hat{\alpha}$ in $S M$ such that

(i) $\hat{\alpha}(0)=v$;

(ii) $q \circ \hat{\alpha}$ is freely homotopic to the concatenation of $\alpha$ with itself a positive (integral) number of times;

(iii) $|\hat{\alpha}| \leq K_{n}|\alpha|^{1 /(n+1)}$ where $n=\operatorname{dim} M$.

Proof of Lemma 3.1.2. Let $A: S_{\alpha(0)} M \rightarrow S_{\alpha(0)} M$ be the holonomy map determined by parallel translation around $\alpha$. For each positive integer $j$, let $\hat{\alpha}_{j}$ be the loop in $S M$ that is the unit length vector field along a curve in $M$ constructed by first parallel translating $v$ around $\alpha, j$ times, and then connecting $A^{j}(v)$ to $v$ by the shortest great circle arc in $S_{\alpha(0)} M$. The loop $\hat{\alpha}$ will be one of these loops $\hat{\alpha}_{j}$ choosen so that (iii) holds ((i) and (ii) hold for all $\hat{\alpha}_{j}$ ). To choose $j$, consider the function $\phi:(0,+\infty) \rightarrow R$ defined by

$$
\phi(x)=|\alpha| x+C_{n} x^{-1 / n},
$$

where $n=\operatorname{dim} M$ and $C_{n}$ comes from Remark 3.1.1. (We may assume that $|\alpha|>0$. If $|\alpha|=0$, then all the $\hat{\alpha}_{j}$ automatically satisfy (iii).) The minimum value $K_{n}^{\prime}|\alpha|^{1 /(n+1)}$ of $\phi$ occurs at $x_{0}$, where

$$
x_{0}=\left(C_{n} / n|\alpha|\right)^{n /(n+1)} \text { and } K_{n}^{\prime}=(n+1)\left(C_{n} / n\right)^{n /(n+1)} \text {. }
$$

Because of Remark 3.1.1, given any positive integer $i$, there exists an integer $j$, with $1 \leq j \leq i$, such that

$$
\left|\hat{\alpha}_{j}\right| \leq|\alpha|(j)+C_{n}(i)^{-1 / n} \leq \phi(i) .
$$

Consequently, letting $i=\left[x_{0}\right]+1$, we see that there exists a positive integer $j$ such that

$$
\left|\hat{\alpha}_{j}\right| \leq 2 \phi\left(x_{0}\right)=2 K_{n}^{\prime}|\alpha|^{1 /(n+1)}
$$

provided $|\alpha| \leq C_{n} / n$. But when $C_{n} / n \leq|\alpha| \leq 1$

$$
\left|\hat{\alpha}_{1}\right| \leq|\alpha|+\pi \leq \pi+1 \leq(\pi+1)\left(C_{n} / n\right)^{-1 /(n+1)}|\alpha|^{1 /(n+1)} .
$$

Consequently, when we let $K_{n}$ be the maximum of

$$
2 K_{n}^{\prime} \text { and }(\pi+1)\left(n / C_{n}\right)^{1 /(n+1)},
$$

$\hat{\alpha}$ can be chosen to satisfy all three properties posited in Lemma 3.1.2.

If $A$ and $B$ are subsets of a metric space $X$, then $d(A, B)$ denotes the distance between these two sets and (by convention) $d(A, \varnothing)=\infty$.

Lemma 3.1.3. Given any pair of positive numbers $\beta$ and $\mu_{0}$, there exists a positive number $\mu_{1}$ (with $\left.\mu_{1}<\mu_{0}\right)$ such that $d\left(M_{\mu_{1}}, M-M_{\mu_{0}}\right) \geq \beta$.

Proof. Consider the geodesic flow $g^{t}$ on $S M$. Since the absolute value of the sectional curvatures of $M$ are bounded from above, there exists a positive 
number $\hat{\beta}$ such that the following inequality holds for any piecewise smooth path $\alpha$ in $S M$ and any (fixed) number $t_{0} \in[0, \beta]$ :

$$
\left|g^{t_{0}} \circ \alpha\right|<\hat{\beta}|\alpha| \text {. }
$$

Let $\alpha$ be any piecewise smooth essential loop in $M$ (with $|\alpha| \leq 1$ ) such that

$$
d\left(\alpha(0), M-M_{\mu_{0}}\right)<\beta .
$$

Then there exists a vector $v \in S_{\alpha(0)} M$ such that $q\left(g^{t_{0}} v\right) \in M-M_{\mu_{0}}$ for some $t_{0} \in[0, \beta)$. Let $\hat{\alpha}$ be the loop in $S M$ that is associated to $\alpha$ and $v$ via Lemma 3.1.2, and $\bar{\alpha}=q \circ g^{t_{0}} \circ \hat{\alpha}$. Then $\bar{\alpha}$ has the following three properties:

(i) $\bar{\alpha}$ is an essential loop in $M$;

(ii) $\bar{\alpha}(0) \in M-M_{\mu_{0}}$;

(iii) $|\bar{\alpha}| \leq \hat{\beta} K_{n}|\alpha|^{1 /(n+1)}$,

where $K_{n}$ comes from Lemma 3.1.2. Since $\bar{\alpha}$ is essential, the other two properties imply that $|\alpha| \geq \hat{\mu}_{0}$ where

$$
\hat{\mu}_{0}=\left(\mu_{0} / \hat{\beta} K_{n}\right)^{n+1} \text {. }
$$

Consequently, $\mu_{1}$ can be any positive number smaller than the minimum of $\frac{1}{2} \hat{\mu}_{0}, \mu_{0}$, and $\frac{1}{2}$.

The next result is a consequence of [32], cf. [3, pp. 110-113].

Lemma 3.2. Assume that $M$ has dimension greater than 1. Let $\bar{M}_{\mu_{0}}$ and $\overline{S M}_{\mu_{0}}$ denote the closures of $M_{\mu_{0}}$ and $(S M)_{\mu_{0}}$ in $M$ and $S M$, respectively. Then $\bar{M}_{\mu_{0}}$ and $\overline{S M}_{\mu_{0}}$ (are either empty or) are codimension-zero submanifolds with nonempty boundaries of $M$ and $S M$, respectively, whose interiors are $M_{\mu_{0}}$ and $(S M)_{\mu_{0}}$. Furthermore, each connected component of $\bar{M}_{\mu_{0}}$ is of one of the following two types:

(i) homeomorphic to $N \times[0,+\infty)$ with $N$ a complete flat Riemannian manifold (where $\pi_{1} N$ injects into $\pi_{1} M$ ), or

(ii) a closed tubular neighborhood $T$ of a simple closed geodesic $\gamma$ whose length $l$ is less than $2 \mu_{0}$.

(Components of type (i) are called "cusp type" components, and those of type (ii) are called "tube type" components.)

We proceed to formulate the main result of this section. A subset $T$ of $S M$ is called a (closed) tube of radius $\varepsilon$ if there exists a closed orbit $\gamma$ of the geodesic flow such that

$$
T=\{y \in S M \mid d(y, \gamma) \leq \varepsilon\}
$$

and $T$ is a closed tubular neighborhood of $\gamma$. (In (3.4), $d($,$) denotes the$ metric on $S M$ determined by the Riemannian metric $\bar{h}($,$) .) The interior of$ $T$ is denoted Int $T$ and is called an open tube. 
We now recall, from [27] and [28], an important technical property of a smooth foliation $\mathscr{F}$ with $j$-dimensional leaves of an $n$-dimensional Riemannian manifold $N^{n}$ whose Riemannian metric is denoted by $\langle,\rangle_{N}$. The main result of the present section verifies this property in an important case. Thus, the foliated control theorem for structure sets, cf. [28], is applicable in this case. In the following definition, $\langle$,$\rangle denotes the standard inner product on R^{n}$.

Definition 3.2.1. Given a pair of positive real numbers $(\beta, \sigma)$, the foliation $\mathscr{F}$ is uniformly $(\beta, \sigma)$-flat on a subset $Y$ of $N^{n}$ provided there exists a positive real number $\delta_{0}$ and a continuous function $r:\left[0, \delta_{0}\right] \rightarrow[0,+\infty)$, with $r(0)=0$ and $r\left(\delta_{0}\right)>1$, such that the following properties are satisfied.

(a) For each point $y$ in $Y$ and each number $\delta$ in the interval $\left(0, \delta_{0}\right)$, there is a smooth chart $g_{y}: B^{j} \times B^{n-j} \rightarrow N$ with $g_{y}(0,0)=y$, where $B^{j}$ and $B^{n-j}$ are the closed balls of radii $\beta$ and $\delta$ centered at the origin in $R^{j}$ and $R^{n-j}$, respectively.

(b) These charts respect the foliation $\mathscr{F}$; i.e., for each $y \in Y$ and each $q \in B^{n-j}, g_{y}\left(B^{j} \times q\right)$ is contained in a leaf of $\mathscr{F}$.

(c) The charts $g_{y}, y \in Y$, satisfy the following Lipschitz inequalities:

$$
\langle v, v\rangle / r\left(\delta_{0}\right) \leq\left\langle d g_{y}(v), d g_{y}(v)\right\rangle_{N} \leq r\left(\delta_{0}\right)\langle v, v\rangle,
$$

where $v$ is any vector tangent to $B^{j} \times B^{n-j}$, and

$$
\langle v, v\rangle / 2 \leq\left\langle d g_{y}(v), d g_{y}(v)\right\rangle_{N} \leq 2\langle v, v\rangle,
$$

when $v$ is tangent to $B^{j} \times q$ for some point $q$ in $B^{n-j}$.

(d) The Taylor expansions of the change of coordinate maps are constrained as follows. Let $y$ and $z$ be any pair of points in $Y$. Then there are smooth maps

$$
\begin{gathered}
u: B^{j} \times B^{n-j} \rightarrow R^{j}, \quad v: B^{n-j} \rightarrow B^{n-j}, \\
I: R^{j} \rightarrow R^{j}, \quad \text { and } \quad L: R^{n-j} \rightarrow R^{n-j}
\end{gathered}
$$

satisfying (d.1), (d.2), and (d.3).

(d.1) $g_{y}^{-1} \circ g_{z}(p, q)=(I(p)+u(p, q), L(q)+v(q))$ for all points $(p, q)$ in the domain of $g_{y}^{-1} \circ g_{z}$.

(d.2) The map $I$ is an isometry, while $L$ is the composite of a linear map $L^{\prime}$ with a translation and $\left|L^{\prime}\right| \leq r\left(\delta_{0}\right)$.

(d.3) For each $q \in B^{n-j}$, let $d u(, q)$ denote the function that assigns to each point $p \in B^{j}$ the matrix of the derivative of the function $u(, q)$ at $p$. Let $d v()$ denote the function that assigns to each $p \in B^{n-j}$ the matrix of the derivative of the function $v()$ at $p$. Then the distances (in the $C^{0}$ metrics) from the functions $u(, q), d u(, q), v()$, and $d v()$ to the corresponding zero maps is less than $\beta \sigma, \sigma, r(\delta) \delta$, and $r\left(\delta_{0}\right) \delta$, respectively. 
Theorem 3.3. Given a positive number $\beta$, there exists a positive number $\mu_{0}$, with $\mu_{0}<\frac{1}{2} \mu$, such that the following statement is true. Given a positive number $\mu_{1}$, with $\mu_{1} \leq \mu_{0}$, there exists an arbitrarily small positive number $\varepsilon$ and a sequence of tubes $T_{i}$ of radius $\varepsilon$ in $S M$ centered about closed orbits $\gamma_{i}$ of the geodesic flow $g^{t}$ such that

(i) the tubes $T_{i}$ are pairwise disjoint;

(ii) $T_{i} \cap \overline{S M}_{\mu_{1}}=\varnothing$, for each index $i$;

(iii) for any positive number $\sigma$, the foliation $\overline{\mathscr{G}}$ of $S M$ is uniformly $(\beta, \sigma)$ flat on $C$, where $C$ is the complement of the union of $(S M)_{\mu_{1}}$ with all the open tubes Int $T_{i}$ in the sequence; i.e.,

$$
C=S M-\left(\left(\bigcup_{i} \operatorname{Int} T_{i}\right) \cup(S M)_{\mu_{1}}\right) ;
$$

(iv) length $\gamma_{i} \in\left[2 \mu_{1}, 2 \beta\right]$.

Theorem 3.3 is a straightforward consequence of the following result.

Lemma 3.4. Given positive real numbers $r_{0}, r_{1}$, and $\varepsilon$ satisfying the inequality $r_{0}<r_{1}$, there exists a positive number $\delta$ such that the following statement is true for any vector $v$ in $S M$. If $d\left(g^{t_{0}} v, v\right)<\delta$ for some number $t_{0} \in\left[r_{0}, r_{1}\right]$, then there exists a vector $v_{0}$ in $S M$ such that

(i) $d\left(g^{t} v, g^{t} v_{0}\right)<\varepsilon$, for all $t \in\left[0, r_{1}\right]$, and

(ii) $g^{t_{1}} v_{0}=v_{0}$

for some number $t_{1} \in\left(0, t_{0}+2 \varepsilon\right)$.

We briefly outline how Lemma 3.4 is used to prove Theorem 3.3, leaving the details to the reader. Pick $\mu_{0}$ sufficiently small so that any closed orbit $\gamma$ of the geodesic flow $g^{t}$ on $S M$, whose length is a number in the interval $\left[2 \mu_{1}, 2 \beta+1\right]$ (where $\mu_{1} \leq \mu_{0}$ ), is disjoint from $\overline{S M}_{\mu_{1}}$. Lemmas 3.2 and 3.1.3 together with the discussion in [3, pp. 110-113] guarantee the existence of $\mu_{0}$. If the length of $\gamma$ is further constrained to lie in the interval $\left[2 \mu_{1}, 2 \beta\right]$, then we show (using Lemma 3.4) that for $\varepsilon$ sufficiently small the set $T$ defined by formula (3.4) is a closed tubular neighborhood of $\gamma$ contained in the complement of $\overline{S M}_{\mu_{1}}$. Furthermore, these neighborhoods are pairwise disjoint as we vary $\gamma$. A final application of Lemma 3.4 verifies Theorem 3.3(iii).

The proof of Lemma 3.4 depends on the following two facts about hyperbolic geometry whose verification is left to the reader. In these remarks, Iso $H^{n}$ denotes the group of all isometries of $H^{n}$. For each semisimple element $\gamma \in$ Iso $H^{n}, \operatorname{Min}(\gamma)$ is the subset of $H^{n}$ defined by

$$
\operatorname{Min}(\gamma) \text { is the set of points where } d_{\gamma}() \text { assumes its minimal }
$$
value. 
Remark 3.4.1. Given a pair of positive real numbers $r_{0}$ and $r_{1}$ with $r_{1}>r_{0}$, there exists a positive number $\varepsilon$ such that for any vector $v \in S H^{n}, d\left(g^{t} v, \gamma^{\prime} v\right)$ $>\varepsilon$ for all $t \in\left[r_{0}, r_{1}\right]$ and any parabolic element $\gamma \in$ Iso $H^{n}$, where $\gamma^{\prime}$ denotes the derivative of $\gamma$.

Remark 3.4.2. Given positive real numbers $r_{0}, r_{1}$, and $\delta$ with $r_{1}>r_{0}$, there exists a positive number $\varepsilon$ such that $d\left(g^{t} v, \gamma^{\prime} v\right)>\varepsilon$ for all $t \in\left[r_{0}, r_{1}\right]$ and any hyperbolic element $\gamma \in$ Iso $H^{n}$ provided $v \in S H^{n}$ satisfies the inequality $d(q v, \operatorname{Min}(\gamma))>\delta$, where $q: S H^{n} \rightarrow H^{n}$ is the canonical bundle projection.

We also use the following result (in proving Lemma 3.4), which is an immediate consequence of the fact that Iso $H^{n}$ acts transitively on $S H^{n}$.

Remark 3.4.3. Given positive numbers $r_{0}, r_{1}$, and $\varepsilon$ with $r_{1}>r_{0}$, there exists a positive number $\delta$ so that the following statement is true. Let $v$ and $w$ be vectors in $S H^{n}$ satisfying

$$
d(q v, q w)<\delta \text { and } d\left(q g^{t_{0}} v, q g^{t_{0}} w\right)<\delta
$$

for some $t_{0} \in\left[r_{0}, r_{1}\right]$. Then $d\left(g^{t} v, g^{t} w\right)<\varepsilon$ for all $t \in\left[0, r_{1}\right]$.

Proof of Lemma 3.4. Suppose $d\left(g^{t_{0}} v, v\right)<\delta$ for some small positive number $\delta$ (how small will be determined in the course of this proof). Let $\bar{v}$ be a vector in $S H^{n}$ that maps to $v$ under the derivative $p^{\prime}$ of the covering projection $p: H^{n} \rightarrow M^{n}=H^{n} / \Gamma$. Then there exists an element $\gamma \in \Gamma$ such that $d\left(g^{t_{0}} \bar{v}, \gamma^{\prime} \bar{v}\right)<\delta$. By picking $\delta$ smaller than the number $\varepsilon$ posited in Remark 3.4.1, we see that $\gamma$ must be a hyperbolic isometry.

Because of Remark 3.4.2, given $\varepsilon_{1}>0$, there exists a vector $w$ in $S H^{n}$ and a positive number $t_{1}$ such that $q w \in \operatorname{Min}(\gamma), d(q \bar{v}, q w)<\varepsilon_{1}$, and $\gamma^{\prime} w=g^{t_{1}} w$, provided $\delta$ is sufficiently small. Since $d(\gamma q \bar{v}, \gamma q w)<\varepsilon_{1}$, we see that

$$
\begin{aligned}
& d\left(q g^{t_{0}} \bar{v}, q g^{t_{1}} w\right)<\delta+\varepsilon_{1},\left|t_{1}-t_{0}\right|<2 \delta+3 \varepsilon_{1} \text {, and conse- } \\
& \text { quently, } d\left(q g^{t_{0}} \bar{v}, q g^{t_{0}} w\right)<2 \delta+3 \varepsilon_{1} .
\end{aligned}
$$

By Remark 3.4.3, we see that if $\varepsilon_{1}$ and $\delta$ are chosen to be sufficiently small, then

$$
t_{1} \in\left[0, t_{0}+2 \varepsilon\right] \text { and } d\left(g^{t} \bar{v}, g^{t} w\right)<\varepsilon
$$

for all $t \in\left[0, r_{1}\right]$. Putting $v_{0}=p^{\prime} w$, one easily completes the proof of Lemma 3.4 by using (3.7).

We will need, in the appendix, that Theorem 3.3 remains valid for any (strictly) negatively curved complete (and connected) locally symmetric space $M$. We now indicate what modifications in the above discussion are needed to verify this. Let $X$ denote the universal covering space of $M$ and $\Gamma$ its group of deck transformations. In particular, $M=X / \Gamma$. As before, $S M$ is the total space of the unit sphere bundle of $M$, and $\overline{\mathscr{G}}$ is the foliation of $S M$ defined by (3.1). The Riemannian metric $\bar{h}($,$) on S M$ is again defined by the formula in [23, p. 547]. The quantities $\operatorname{Inj} \operatorname{Rad}(x), d_{\gamma}(y)$, and $d_{\Gamma}(y)$ (where 
$x \in M, y \in X$, and $\gamma \in \Gamma$ ) are likewise defined by (3.2), and $\mu$ will again denote the positive number $\mu(n)$ posited in Margulis' Lemma (cf. [3, Theorem, p. 101]), where $n=\operatorname{dim} X$. We have normalized the metric on $X$ so that all its sectional curvatures satisfy the inequalities $-1 \leq K<0$. The sets $M_{\mu_{0}}$ and $(S M)_{\mu_{0}}$ (where $0<\mu_{0}<\frac{1}{2} \mu$ ) are defined by the formulas in (3.3), and Iso $(X)$ denotes the Lie group consisting of all isometries of $X$. It is well known that Iso $(X)$ contains no "mixed" isometries; i.e., any parabolic isometry is strictly parabolic. (See [3, p. 77] for terminology.) Then both Lemmas 3.1.3 and 3.2 remain true but with statement (i) of Lemma 3.2 modified as follows:

The connected component has the form $N \times[0,+\infty)$ where $N$ is an aspherical manifold (without boundary) whose fundamental group is virtually polycyclic and injects into $\pi_{1} M$.

(The fact that $\pi_{1} N$ is virtually polycyclic follows from the discussion in [3, $\S 10.3]$ and [48, Lemma 2.2].)

Using the fact that Iso $(X)$ acts transitively on $S X$, one can show that Remarks 3.4.1-3.4.3 remain valid when, in their statements, Iso $\left(H^{n}\right)$ is replaced with Iso $(X)$. Since Lemma 3.4 is a consequence of these remarks and Theorem 3.3 is a consequence of Lemma 3.4, we see that they both remain valid (without change in their statements) for an arbitrary negatively curved complete (connected) locally symmetric space $M$.

This finishes our first digression. We now return to the situation where $M$ denotes a complete (connected) real hyperbolic manifold. We do need a second analogue of Theorem 3.3, namely, where $S M$ is replaced by $P M$. We proceed to formulate it. Let $q: P M \rightarrow M$ denote the bundle projection and define a subset $(P M)_{\mu_{0}}$, for each positive number $\mu_{0}<\frac{1}{2} \mu$, by the equation

$$
(P M)_{\mu_{0}}=q^{-1}\left(M_{\mu_{0}}\right) \text {. }
$$

Let $\gamma$ be a closed leaf of the foliation $\mathscr{G}$ of $P M$ (recall that $\gamma$ is homeomorphic to $S^{1}$ ). Then a subset $T$ of $P M$ is called a (closed) tube of radius $\varepsilon$ (centered about $\gamma$ ) if

$$
T=\{y \in P M \mid d(y, \gamma) \leq \varepsilon\}
$$

and $T$ is a closed tubular neighborhood of $\gamma$. (In (3.8.2), $d($,$) denotes the$ metric on $P M$ determined by the restriction of the Riemannian metric $\hat{h}$ on $F M$ to $P M$. Note that the covering projection $S M \rightarrow P M$ is a local isometry.) The interior of $T$ is denoted by Int $T$ and is called an open tube. The proof of Theorem 3.3 is easily modified to yield the following result.

Addendum 3.4.4. Theorem 3.3 remains valid when in its statement $S M$, $(S M)_{\mu_{1}}, \overline{\mathscr{G}}$ are replaced by $P M,(P M)_{\mu_{1}}, \mathscr{G}$, respectively, and the phrase "closed orbits $\gamma_{i}$ of the geodesic flow $g^{t}$ " is replaced with "closed leaves $\gamma_{i}$ of $\mathscr{G}$.

The next result is an immediate consequence of Addendum 3.4.4. 
Corollary 3.5. Let $K$ be a compact subset of $P M$ and $\beta$ a positive number such that any closed leaf of $\mathscr{G}$ that meets $K$ has length greater than $2 \beta$. Then the foliation $\mathscr{G}$ is uniformly $(\beta, \sigma)$-flat on $K$ for any positive number $\sigma$.

We need a third and final digression. For this we return to the situation where $M$ denotes any (strictly) negatively curved complete (and connected) locally symmetric space. Let $H M$ denote the enlargement of $M$ that was defined in [23, p. 542]. It is a special case of the warped product construction due to Bishop and O'Neill in [4, §7]. As a smooth manifold, $H M$ is $M \times(-1,1)$. The Riemannian metric $B($,$) on H M$ is defined by [23, (2.1)]. Consider the diffeomorphism $\tau: M \times R \rightarrow M \times(-1,1)$ defined by

$$
\tau(x, t)=(x, \sinh (\tanh t)) \text {, }
$$

where $x \in M$ and $t \in R$. Let $\widehat{B}($,$) be the Riemannian metric on M \times R$ such that $\tau$ is an isometry. $\widehat{B}($,$) is explicitly described in the following$ formula:

$$
\widehat{B}_{(x, t)}(X, Y)=(\cosh (\sinh t))^{2} b_{x}\left(X_{0}, Y_{0}\right)+(\cosh t)^{2} r s,
$$

where $X, Y$ are vectors tangent to $M \times R$ at $(x, t)$ and $X_{0}, Y_{0}, r, s$ are the obvious "components" of $X, Y$ defined by [23, (2.2) and (2.4)]. The manifold $M \times R$ equipped with $\widehat{B}($,$) is called the expansive model of the enlargement$ of $M$ because of the following consequence of (3.8.4).

Remark 3.5.1. There exists a positive number $c$ such that $\widehat{B}(X, X) \geq$ $|t| P(X, X)$ for any vector $X$ tangent to $M \times R$ at a point $(x, t)$ provided $|t| \geq c$. Here $P($,$) denotes the product of the given Riemannian metric on$ $M$ with the Euclidean metric on $R$.

Let $S^{+} H M$ denote the asymptotic northern hemisphere of the unit sphere bundle $S H M$ (recall that $S^{+} H M$ is a subspace of $S H M$ ). It is defined by [23, (0.12)]. Let $q: S^{+} H M \rightarrow H M=M \times R$ denote the bundle projection. Before stating our final digression, we must formulate some terminology. The geodesic flow $g^{t}$ on $S H M$ leaves $S^{+} H M$ invariant, and all its closed orbits $\gamma$ lie in $\partial\left(S^{+} H M\right)$ (see [23, p. 547]). If $T$ is a tube of radius $\varepsilon$ (in $S H M$ ) about $\gamma$, then $T \cap S^{+} H M$ is also called a tube of radius $\varepsilon$ (in $S^{+} H M$ ) provided the pair $\left(T \cap S^{+} H M, T \cap \partial\left(S^{+} H M\right)\right)$ is homeomorphic to the pair $(\mathscr{T} \times[0,1], \mathscr{T} \times$ $0)$, where $\mathscr{T}$ is a (closed) tubular neighborhood of $\gamma$ in $\partial\left(S^{+} H M\right)$. The corresponding open tube refers to Int $T \cap S^{+} H M$ and, by abuse of notation, will sometimes be denoted Int $T^{\prime}$, where $T^{\prime}=T \cap S^{+} H M$. The foliation of $S^{+} H M$, whose leaves are the orbits of $g^{t}$, will still be denoted by $\overline{\mathscr{G}}$. If $C$ is a subset of $S^{+} H M$, the notion of $\overline{\mathscr{G}}$, being uniformly $(\alpha, \sigma)$-flat on $C$, is the obvious generalization to a foliation of a manifold with boundary of Definition 3.2.1.

The following modified version of Lemma 3.4 is true for $S H M$.

Addendum 3.5.2. Fixing a positive real number $c$, Lemma 3.4 remains valid when the following changes are made in its statement. Replace $S M$ with 
$S H M$, where $M$ denotes a complete, connected (strictly) negatively curved locally symmetric space. We additionally constrain the vector $v$ to be tangent to $H M=M \times R$ at a point in $M \times[-c, c]$.

This addendum is a consequence of the generalized version of Lemma 3.4 mentioned in our first digression together with the following two additional facts. Let $X$ denote the symmetric space that is the total space of the universal cover of $M$.

Remark 3.5.3. For any positive $c$, Iso $X$ acts cocompactly on $q^{-1}(X \times[-c, c])$.

Let $f: X \times R \rightarrow R$ denote projection onto the second factor. Using Remark 3.5.3, the next result is analogous to Remark 3.6.

Remark 3.5.4. Given positive real numbers $c, r_{0}, r_{1}$, and $\delta$ with $r_{1}>r_{0}$, there exists a positive number $\varepsilon$ such that $\left|d f\left(g^{t} v\right)-d f(v)\right|>\varepsilon$ for all $t \in$ $\left[r_{0}, r_{1}\right]$, provided either $|d f(v)| \geq \delta$ or $v$ is tangent to $H X=X \times R$ at a point $x$ such that $\delta \leq|f(x)| \leq c$.

As Theorem 3.3 was a consequence of Lemma 3.4, so our third (and final) digression is a consequence of Addendum 3.5.2.

Addendum 3.5.5. Fixing a positive number $c$, Theorerm 3.3 remains valid when the following changes are made in its statement. Replace $S M$ with $S^{+} H M$, where $M$ denotes a complete, connected (strictly) negatively curved locally symmetric space. Replace $\overline{S M}_{\mu_{1}}$ with $q^{-1}\left(\bar{M}_{\mu_{1}} \times R\right)$ and redefine $C$ by the new formula

$$
C=(f \circ q)^{-1}([-c, c])-\left(\left(\bigcup_{i} \operatorname{Int} T_{i}\right) \cup q^{-1}\left(\bar{M}_{\mu_{1}} \times R\right)\right) .
$$

Recall that $q: S^{+} H M \rightarrow H M$ is the bundle projection and $f: H M=M \times R \rightarrow$ $R$ is projection onto the second factor.

Theorem 3.6. Given a compact subset $K$ of $M$ and a pair of positive numbers $\beta$ and $\sigma$, there exists a positive number $r_{0}$ such that the following statement is true. For any number $r$, with $r \geq r_{0}$, the foliation $\hat{\mathscr{G}}_{r}$ is uniformly $(\beta, \sigma)$-flat on $q^{-1}(K) \cap \widehat{\mathscr{D}}_{r} M$, where $q: F M \rightarrow M$ denotes the bundle projection. Recall that $\widehat{\mathscr{D}}_{r} M$ is the shell of radius $r$ about its core PM (measured in the primary leaf metric (see Definition 2.7)).

Before we can prove Theorem 3.6, we need some preliminary results. Given a compact subset $K$ of $M$ and a real number $r$, let $K_{r}$ denote the subset of $M$ defined by the equation

$$
K_{r}=\{x \mid x \in M, d(x, K) \leq r\} .
$$

Since $K_{5 \beta}$ is compact, there is only a finite number of free homotopy classes of loops in $M$ represented by piecewise smooth loops $\alpha$ in $K_{5 \beta}$, where $|\alpha|<5 \beta$. Consequently, there exists a positive number $\mu_{1}$, with $\mu_{1}<\mu_{0}$, such that the following statement is true. 
Remark 3.7. Let $\alpha$ and $\hat{\alpha}$ be freely homotopic piecewise smooth loops in $M$ and $\tau$ be a positive number satisfying

(i) image $\alpha$ is contained in $K_{5 \beta}$,

(ii) $|\alpha|<5 \beta$, and

(iii) $|\hat{\alpha}|<\tau<\mu_{1}$.

Then image $\hat{\alpha}$ is contained in a "cusp type" component of $\bar{M}_{\tau}$.

Pick a positive number $\mu_{2}<\mu_{1}$ (using Lemma 3.1.3) such that

$$
d\left(M_{\mu_{2}}, M-M_{\mu_{1}}\right)>5 \beta,
$$

and note that this implies that

$$
d\left(\mathscr{C}_{1}, \mathscr{C}_{2}\right)>5 \beta
$$

for any pair of distinct components $\mathscr{C}_{1}, \mathscr{C}_{2}$ of $\bar{M}_{\mu_{2}}$. We say that a piecewise smooth path $\alpha:[0,1] \rightarrow P M$ is $\left(\beta^{\prime}, \delta^{\prime}\right)$-controlled relative to $\mathscr{G}$ if there exists a piecewise smooth path $\eta:[0,1] \rightarrow P M$ such that

(i) image $\eta$ is contained in a leaf of $\mathscr{G}$;

(ii) $|\eta|<\beta^{\prime}$;

(iii) $d(\eta(t), \alpha(t))<\delta^{\prime}$ for all $t \in[0,1]$.

The following is clearly true.

Remark 3.8. Given a uniform $(\beta, \sigma)$-flatness structure for the foliation $\mathscr{G}$ on a subset $Y$ of $P M$, there exists a positive number $\delta^{\prime}$ such that any piecewise smooth path $\alpha$ in $Y$, which is $\left(\frac{1}{2} \beta, \delta^{\prime}\right)$-controlled relative to $\mathscr{G}$, is contained in the image of some chart of the structure.

We will now construct a family of charts for $\widehat{\mathscr{D}}_{r} H^{n}$, which we will later use in proving Theorem 3.6. One easily finds a smooth embedding $\hat{\phi}: U \times B_{2} \rightarrow \widehat{\mathscr{D}}_{1} H^{n}$ having the following properties:

(i) $B_{2}$ is a closed ball in $R^{2 n-2}$ centered at 0 (let $\rho_{2}$ denote the radius of $\left.B_{2}\right)$ and $U=U_{0} \times U_{1}$, where $U_{0}$ and $U_{1}$ are open subsets of $S^{n-2}$ and $R$, respectively, and $S^{n-2}$ denotes the $(n-2)$-dimensional sphere of radius $\overline{1}$, where $\sqrt{2} \geq \overline{1} \geq 1 / \sqrt{2}$ (cf. Lemma 2.9).

(ii) For each point $y \in B_{2}, \hat{\phi}(U \times y)$ is contained in some leaf $L_{y}$ of $\hat{\mathscr{G}}_{1}$, and $\left.\hat{\phi}\right|_{\bar{U} \times y}$ is an isometry onto an open subset of $L_{y}$. 
Let $\bar{\phi}: B \rightarrow U$ be a smooth codimension-zero embedding, where $B=B_{0} \times B_{1}$, satisfying

(i) $B_{0}$ and $B_{1}$ are closed balls centered at 0 in $R^{n-2}$ and $R$, respectively (let $\rho_{i}$ denote the radius of $B_{i}$, where $i=0$ or 1$)$;

(ii) $\bar{\phi}=\bar{\phi}_{0} \times \bar{\phi}_{1}$, where $\bar{\phi}_{0}: B_{0} \rightarrow U_{0}$ and $\bar{\phi}_{1}: B_{1} \rightarrow U_{1}$;

(iii) $d \bar{\phi}_{0}$ and $d \bar{\phi}_{1}$ are both isometries at 0 .

Let $\phi: B \times B_{2} \rightarrow \widehat{\mathscr{D}}_{1} H^{n}$ be the composite of $\hat{\phi}$ and $\bar{\phi}$; i.e.,

$$
\phi=\hat{\phi} \circ \bar{\phi} .
$$

For each element $g \in$ Iso $H^{n}$, consider the composite chart

$$
g \circ \phi: B \times B_{2} \rightarrow \widehat{\mathscr{D}}_{1} H^{n} \text {. }
$$

The following result is easily proved.

Lemma 3.9. Given a positive number $\sigma$, there exists a positive number $\beta$ such that the charts $\left\{g \circ \phi \mid g \in\right.$ Iso $\left.H^{n}\right\}$ determine a uniform $(\beta, \sigma)$-flatness structure for the foliation $\mathscr{G}_{1}$ on all of $\widehat{\mathscr{D}}_{1} H^{n}$ by restricting the domain $B \times B_{2}$ of the charts to a Cartesian product of two concentric smaller balls. (Recall that $B=B_{0} \times B_{1}$ is not a ball, but each $B_{i}$ is.)

To construct charts on $\widehat{\mathscr{D}}_{r} H^{n}$, we must first consider the natural diffeomorphism $f_{r}: \widehat{\mathscr{D}}_{1} H^{n} \rightarrow \mathscr{D}_{r} H^{n}$ defined as follows. Let $L$ be the leaf of $\mathscr{F}$, equipped with its secondary Riemannian metric, containing a point $x \in \widehat{\mathscr{D}}_{1} H^{n}$ and $l$ the line that marks $L$. Then

$$
\begin{aligned}
& f_{r}(x)=y, \text { provided } y \in L \cap \widehat{\mathscr{D}}_{r} H^{n} \text { and a geodesic line in } L \\
& \text { containing } x \text { and } y \text { meets } l \text { perpendicularly at a point which } \\
& \text { is not on the segment between } x \text { and } y .
\end{aligned}
$$

Since $L \cap \widehat{\mathscr{D}}_{1} H^{n}$ is isometrically identifiable to the Riemannian product $S^{n-2} \times$ $R, f_{r}$ has the following dilation property relative to such an identification, provided $n>3$.

Remark 3.10. The diffeomorphism $f_{r}$ is Iso $H^{n}$-equivariant and satisfies the following equalities and inequalities, where $u$ is any vector tangent to $S^{n-2} \times t$ for some $t \in R$ and $v$ is any vector tangent to $y \times R$ for some $y \in S^{n-2}$ :

$$
\left|d f_{r}(u)\right|=a_{r}|u| \text { and }\left|d f_{r}(v)\right|=b_{n}|v|,
$$

where $a_{r}$ and $b_{r}$ satisfy

$$
\frac{1}{\sqrt{2}} \frac{\cosh \hat{r}}{\cosh \hat{1}} \leq b_{n} \leq \sqrt{2} \frac{\cosh \hat{r}}{\cosh \hat{1}}
$$

and

$$
\frac{1}{\sqrt{2}} \frac{\sinh \hat{r}}{\sinh \hat{\imath}} \leq a_{n} \leq \sqrt{2} \frac{\sinh \hat{r}}{\sinh \hat{1}},
$$


where $r \mapsto \hat{r}$ is the function posited in Lemma 2.8. This remark can be readily verified using the argument given in the proof of Lemma 2.9.

Let $\psi_{r}: \mathscr{B}_{r} \times B_{2} \rightarrow B \times B_{2}$ be defined by

$$
\begin{gathered}
\psi_{r}(x, y, z)=\left(x / a_{r}, y / b_{r}, z\right), \\
\text { where } \mathscr{B}_{r}=\left\{(x, y) \in R^{n-2} \times R|| x\left|\leq a_{r} \rho_{0},\right| y \mid \leq b_{n} \rho_{1}\right\} .
\end{gathered}
$$

The following result is a consequence of Lemma 3.9 and Remark 3.10.

Lemma 3.11. Given positive numbers $\beta$ and $\sigma$, there exists a positive number $r_{0}$ such that for any number $r \geq r_{0}$ the charts $\left\{g \circ f_{r} \circ \phi \circ \psi_{r} \mid g \in\right.$ Iso $\left.H^{n}\right\}$ determine (by restricting the domain $\mathscr{B}_{r} \times B_{2}$ of the charts) a uniform $(\beta, \sigma)$ flatness structure for the foliation $\hat{\mathscr{G}}_{r}$ on all of $\widehat{\mathscr{D}}_{r} H^{n}$.

Let $p: \widehat{\mathscr{D}}_{r} H^{n} \rightarrow \widehat{\mathscr{D}}_{r} M$ denote the covering projection and $B_{2}(\delta), B(\delta)$ denote the closed balls of radius $\delta>0$ centered at 0 in $R^{2 n-2}, R^{n-1}$, respectively. (Note that if $\delta \leq \rho_{2}$, then $B_{2}(\delta)$ is a subset of $B_{2}$ and $B(\beta) \subset \mathscr{B}_{r}$ if $r>r_{0}$.) For each element $g$ in Iso $H^{n}$ and each positive number $\delta \leq \delta_{0}$, let $C_{g}(\delta)$ be the subset of $\widehat{\mathscr{D}}_{r} H^{n}$ defined by

$$
C_{g}(\delta)=g \circ f_{r} \circ \phi \circ \psi_{r}\left(B(\beta) \times B_{2}(\delta)\right) .
$$

For each subset $S$ of $\widehat{\mathscr{D}}_{r} M$ and any positive number $\delta \leq \delta_{0}$, define a subset Iso $\left(H^{n}, S, \delta\right)$ of Iso $H^{n}$ by

$$
\text { Iso }\left(H^{n}, S, \delta\right)=\left\{g \in \text { Iso } H^{n} \mid C_{g}(\delta) \cap p^{-1}(S) \neq \varnothing\right\} .
$$

With this notation, we have the following immediate consequence of Lemma 3.11 .

Addendum 3.12. Let $S$ be a subset of $\widehat{\mathscr{D}}_{r} M$, where $r \geq r_{0}$ and $r_{0}$ is the number posited in Lemma 3.11 and dependent on the pair of positive numbers $(\beta, \sigma)$. If there exists a positive number $\delta \leq \delta_{0}$ such that $C_{g}(\delta) \cap \gamma C_{g}(\delta)=\varnothing$ for all $\gamma \in \Gamma-\{$ id $\}$ and all $g \in \operatorname{Iso}\left(H^{n}, S, \delta\right)$, then we obtain a uniform $(\beta, \sigma)$-flatness structure for the foliation $\hat{\mathscr{G}}_{r}$ on $S$ by restricting the domain $\beta_{r} \times B_{2}(\delta)$ of the maps in the set

$$
\left\{p \circ g \circ f_{r} \circ \phi \circ \psi_{r} \mid g \in \operatorname{Iso}\left(H^{n}, S, \delta\right)\right\} .
$$

Proof of Theorem 3.6. Pick a number $\bar{r}_{0}$ that is bigger than the number $r_{0}$ of Lemma 3.11 and satisfies the inequality

$$
\cosh \left(\bar{r}_{0} / \sqrt{2}\right)>100 \beta / \mu_{2},
$$

where $\mu_{2}$ comes from (3.9.1). Then $\bar{r}_{0}$ is the number " $r_{0}$ " whose existence is posited in Theorem 3.6. Now fix a number $r$ satisfying $r \geq \bar{r}_{0}$, and let the sets $S$ and $S^{\prime}$ be defined by

$$
S=q^{-1}(K) \cap \widehat{\mathscr{D}}_{r} M \quad \text { and } \quad S^{\prime}=q^{-1}\left(K_{5 \beta}\right) \cap \widehat{\mathscr{D}}_{r} M .
$$


Since $S^{\prime}$ is compact, there exists a positive number $\mu_{3}\left(\right.$ with $\left.\mu_{3}<\mu_{2}\right)$ such that

$$
q \circ p_{d}\left(S^{\prime}\right) \cap M_{\mu_{3}} \text { is the empty set. }
$$

Let $\check{M}_{\mu_{2}}$ denote the union of all the "cusp type" components of $\bar{M}_{\mu_{2}}$ and $Y$ denote the subset of $P M$ defined by

$$
Y=q^{-1}\left(\check{M}_{\mu_{2}}-M_{\mu_{3}}\right) \cap P M .
$$

Applying Addendum 3.4.4 (in which $\mu_{1}$ is replaced by $\mu_{3}$ ), we see that the foliation $\mathscr{G}$ of $P M$ is uniformly $(\beta, \sigma)$-flat on $Y$. Let $\delta^{\prime}$ be the positive number determined by this flatness structure via Remark 3.8. Using (3.18.1) and the fact that $S^{\prime}$ is compact, a continuity argument yields the existence of a positive number $\delta$ (where $\delta$ is smaller than the number $\delta_{0}$ in Addendum 3.12 ) so that the following is true.

Remark 3.13. If $C_{g}(\delta) \cap \gamma C_{g}(\delta)$ is not empty for some $g \in \operatorname{Iso}\left(H^{n}, S, \delta\right)$ and some $\gamma \in \Gamma-\{$ id $\}$, than there exists a piecewise smooth loop $\alpha:[0,1] \rightarrow \widehat{\mathscr{D}}_{r} M$ such that

(i) $|\alpha|<5 \beta$;

(ii) $q \circ \alpha$ is an essential loop in $M$;

(iii) $\left|p_{d} \circ \alpha\right|<\frac{1}{2} \mu_{2}$;

(iv) $p_{d} \circ \alpha$ is $\left(\frac{1}{2} \beta, \delta^{\prime}\right)$-controlled relative to $\mathscr{G}$;

(v) image $\alpha$ is contained in $S^{\prime}$.

We now deduce Theorem 3.6 from Addendum 3.12 by showing that its hypotheses are satisfied for the number $\delta$ in Remark 3.13. If not, then there exists a loop $\alpha$ satisfying Remark 3.13(i)-(v). Observe that the loops $q \circ \alpha$ and $q \circ p_{d} \circ \alpha$ are freely homotopic (via the homotopy $q \circ r_{t} \circ \alpha$, where $r_{t}: \mathscr{D} M \rightarrow$ $\mathscr{D} M$ is the radial deformation retraction of $\mathscr{D} M$ onto $P M$ with $r_{1}=p_{d}$ ). Consequently, $q \circ p_{d} \circ \alpha$ is an essential loop in $M$. Also, $\left|q \circ p_{d} \circ \alpha\right|<\frac{1}{2} \mu_{2}$ and $|q \circ \alpha|<5 \beta$ since $d_{q}$ is distance nonincreasing. Remark 3.4.3 together with these two facts and (3.18.3) shows that image $p_{d} \circ \alpha$ is contained in $Y$. Remark 3.8 together with Remark 3.13(iv) now yields that image $p_{d} \circ \alpha$ is contained in the image of some chart. Consequently, $p_{d} \circ \alpha$ and, therefore, $q \circ p_{d} \circ \alpha$ are null homotopic loops. This contradiction completes the proof of Theorem 3.6.

We use a variant of Theorem 3.6 in proving our main result. We now formulate this variant. Note that $H M$ is a real hyperbolic manifold since $M$ is; cf. [23, Lemma 2.1]. Let $\bar{F} H M$ denote the codimension-two submanifold of $F H M$ consisting of all unordered pairs $\left[v_{1}, v_{2}\right]$, where each $v_{i} \in \partial S^{+} H M$. Recall that $\partial S^{+} H M$ is the total space of the asymptotic equator bundle of $S H M$ (cf. [23, p. 545]). Let $\overline{\mathscr{D}}_{r} H M$ denote the intersection of $\bar{F} H M$ and $\widehat{\mathscr{D}}_{r} H M$; i.e.,

$$
\overline{\mathscr{D}}_{r} H M=\bar{F} H M \cap \widehat{\mathscr{D}}_{r} H M .
$$


Consider the fiber bundle projection

$$
p_{d}: \widehat{D}_{r} H M \rightarrow P H M
$$

described by (1.15) and Lemmas 2.6 and 2.8. Note that $p_{d}^{-1}(P M)=\overline{\mathscr{D}}_{r} H M$, where we consider $P M$ to be a subset of $P H M$ by identifying $M$ with $M \times 0$ in $H M=M \times R$. Thus,

$$
p_{d}: \overline{\mathscr{D}}_{r} H M \rightarrow P M
$$

is a fiber bundle that is the restriction of the bundle in (3.20) to the codimensiontwo submanifold $P M$ of $P H M$. Consequently, $\overline{\mathscr{D}}_{r} H M$ is a codimension-two submanifold of $\widehat{\mathscr{D}}_{r} H M$. Each leaf of the foliation $\hat{\mathscr{G}}_{r}$ that meets $\overline{\mathscr{D}}_{r} H M$ is totally contained in $\overline{\mathscr{D}}_{r} H M$; therefore, these leaves determine a foliation of $\overline{\mathscr{D}}_{r} H M$, which we denote by $\overline{\mathscr{G}}_{r}$. Hence, the charts posited in Theorem 3.6 for the foliation $\widehat{\mathscr{G}}_{r}$ of $\widehat{\mathscr{D}}_{r} H M$ can be used to induce uniform flatness structures for the foliations $\overline{\mathscr{G}}_{r}$ on the relevant subsets of $\overline{\mathscr{D}}_{r} H M$. In particular, we have the following variant of Theorem 3.6.

Corollary 3.13.1. Given a compact subset $K$ of $H M$ and a pair of positive numbers $\beta$ and $\sigma$, there exists a positive number $r_{0}$ such that the following statement is true. For any number $r$, with $r \geq r_{0}$, the foliation $\overline{\mathscr{G}}_{r}$ is uniformly $(\beta, \sigma)$-flat on $q^{-1}(K) \cap \overline{\mathscr{D}}_{r} H M$, where $q: \bar{F} H M \rightarrow H M$ denotes the bundle projection.

Let $P^{\prime} M$ be the submanifold of $\bar{F} H M$ consisting of all unordered pairs of vectors $[X, Y]$ tangent to $H M=M \times R$ such that $X_{0}=-Y_{0}$, where we use the notation of [23, p. 542]; i.e., if $X$ is tangent to $M \times R$, then $X_{0}=d p_{1}(X)$, where $p_{1}$ and $p_{2}$ denote the projections onto the first and second factors of $M \times R$, respectively. Note that $P^{\prime} M$ contains $P M$; in fact, $P^{\prime} M \cap F M=P M$, where we identify (as before) $M$ as $M \times 0$ inducing identifications of $P M$ and $F M$ to subspaces of $\bar{F} H M$. Let $\tau$ denote the open subset of $\bar{F} H M$, which is the complement of $P^{\prime} M$; i.e.,

$$
\tau=\bar{F} H M-P^{\prime} M,
$$

and define a smooth retraction

$$
\rho_{\tau}: \tau \rightarrow \partial S^{+} H M
$$

as follows: $\rho_{\tau}[X, Y]=Z$, where

$$
\frac{Z_{0}}{\left|Z_{0}\right|}=\frac{X_{0}+Y_{0}}{\left|X_{0}+Y_{0}\right|} \quad \text { and } \quad d p_{2}(Z)=d p_{2}(X)=d p_{2}(Y) \text {. }
$$

Here we identify $\partial S^{+} H M$ with the set of unordered pairs $[X, X]$, where $X \in$ $\partial S^{+} H M$. (Note that if $X$ and $Y$ are vectors in $\partial S^{+} H M$ that are tangent to $M \times R$ at points $x$ and $y$, respectively, and such that $p_{2}(x)=p_{2}(y)$, then $d p_{2}(X)=d p_{2}(Y)$.) 
Let $\gamma$ denote the complement of $\partial S^{+} H M$ in $\bar{F} M$; i.e.,

$$
\gamma=\bar{F} H M-\partial S^{+} H M \text {. }
$$

Let $\rho_{\gamma}: \gamma \rightarrow P M$ denote the bundle projection that is the restriction of

$$
p_{d}: F H M-S H M \rightarrow P H M
$$

to $\gamma$.

Given a positive number $\varepsilon$, define the subsets ${ }_{\varepsilon} \gamma$ (and ${ }_{\varepsilon} \dot{\gamma}$ ) of $\gamma$ to consist of all points $x$ such that the distance from $x$ to $\rho_{\gamma}(x)$, measured in $\rho_{\gamma}^{-1}\left(\rho_{\gamma}(x)\right)$, is less than or equal to $\varepsilon$ (and equal to $\varepsilon$ ), respectively. Similarly, we define the subsets ${ }_{\varepsilon} \tau$ (and ${ }_{\varepsilon} \dot{\tau}$ ) of $\tau$, using $\rho_{\tau}$ in place of $\rho_{\gamma}$. If $\delta$ and $\varepsilon$ are a pair of positive numbers with $\delta<\varepsilon$, define sets ${ }_{\delta, \varepsilon} \gamma$ and ${ }_{\delta, \varepsilon} \tau$ to be the closures of $\left({ }_{\varepsilon} \gamma\right)-\left({ }_{\delta} \gamma\right)$ and $\left({ }_{\varepsilon} \tau\right)-\left({ }_{\delta} \tau\right)$, respectively.

The following three facts are easily verified.

Lemma 3.14. (i) Given positive numbers $\beta$ and $\delta$, there exists a positive number $\alpha$ such that

$$
{ }_{\delta} \tau \cup{ }_{\alpha} \gamma \supseteq q^{-1}(M \times[-\beta, \beta]),
$$

where $q: \bar{F} H M \rightarrow H M=M \times R$ is the bundle projection.

(ii) Given a positive number $\alpha$, there exists a positive number $\delta$ such that ${ }_{\delta} \tau \cap_{\alpha} \gamma=\varnothing$.

(iii) Given positive numbers $\beta, \varepsilon$, and $\delta$, there exist positive numbers $\varepsilon^{\prime}$ and $\alpha$ such that

$$
{ }_{\delta} \tau \cup\left({ }_{\alpha} \gamma \cap\left(\bar{q} \circ \rho_{\gamma}\right)^{-1}\left(M-M_{\varepsilon^{\prime}}\right)\right) \supseteq q^{-1}\left(\left(M-M_{\varepsilon}\right) \times[-\beta, \beta]\right),
$$

where $\bar{q}: P M \rightarrow M$ is the bundle projection.

We end this rather long section with two final comments. The first is a local flatness statement for the foliation $\mathscr{F}$ of the manifold $M$, where the leaves of $\mathscr{F}$ are the points of $M$. (Note that a closed ball of radius $\beta>0$ in $R^{0}$ is a single point.) In this result, $M$ is any (strictly) negatively curved complete (and connected) locally symmetric space. Let $X$ denote the total space of the universal cover of $M$. Using the fact that Iso $X$ acts transitively on $X$, the following result is obviously true.

Lemma 3.15. Given positive real numbers $\beta, \sigma$, and $\varepsilon$, the foliation $\mathscr{F}$ is uniformly $(\beta, \sigma)$-flat on $M-M_{\varepsilon}$.

We now return to the situation where $M$ is restricted to be a complete (connected) real hyperbolic manifold. To each positive number $r$, we associate the manifold $W_{r}$ defined by

$$
W_{r}=\overline{\mathscr{D}}_{r} H M \times(0,+\infty)
$$

equipped with the product of the Riemannian metric on $\overline{\mathscr{D}}_{r} H M$ and the Euclidean metric on $(0,+\infty) . W_{r}$ has a foliation $\mathscr{F}_{r}$ whose leaves are Cartesian 
products $L \times(0,+\infty)$, where $L$ is a leaf of $\overline{\mathscr{G}}_{r}$. Corollary 3.13 .1 has the following consequence.

Addendum 3.15.1. Given a compact subset $K$ of $H M$ and a pair of positive numbers $\beta$ and $\sigma$, there exists a positive number $r_{0}$ such that the following statement is true. For any number $r$, with $r \geq r_{0}$, the foliation $\mathscr{F}_{r}$ is uniformly $(\beta, \sigma)$-flat on the set $\left(q^{-1}(K) \cap \overline{\mathscr{D}}_{r} H M\right) \times(0,+\infty)$, where $q: \bar{F} H M \rightarrow H M$ denotes the bundle projection.

There is a natural diffeomorphism

$$
\bar{f}_{r}: W_{r} \rightarrow \bar{F} H M-\left(P M \cup \partial S^{+} H M\right)
$$

defined by the equation

$$
\bar{f}_{r}(x, t)=f_{t}\left(f_{r}^{-1}(x)\right),
$$

where $f_{t}$ was defined in (3.15) (cf. (3.19)). Notice that if $L$ is a leaf of $\mathscr{F}_{r}$, then $\bar{f}_{r}(L)$ is contained in a leaf of the foliation $\mathscr{F}$ of $F H M$. We now state the final results of this section. It is a consequence of Corollary 2.2 and Lemma 2.4 .

Lemma 3.16. Given a positive real number $\alpha$, there exists a real number $\beta$, with $\beta \geq 1$, such that

$$
\beta|v| \geq\left|d f_{r}(v)\right| \geq|v| / \sqrt{2}
$$

provided $v$ is tangent to a leaf of the foliation $\mathscr{F}_{r}$ at a point $(x, t)$ in $\overline{\mathscr{D}}_{r} H M \times$ $(0,+\infty)$, where $r+\alpha \geq t \geq r$ (and $r \geq \alpha$ ). When $\alpha \geq 1, \beta$ can be taken to be $e^{3 \alpha}$.

Some of the constructions of $\S \S 1-3$ are denoted differently in $\S \S 4-9$ and the appendix. We end $\S 3$ with a table listing the notation of $\S \S 4-9$ and $A$ in terms

\begin{tabular}{|c|c|c|c|}
\hline & Construction & Notation of $\S \S 4-9$ and $A$ & Notation of $\S \S 1-3$ \\
\hline 1. & $\begin{array}{l}\text { Asymptotic northern } \\
\text { hemisphere bundle of } H M^{\prime}\end{array}$ & $D(T(M))$ & $S^{+} H M^{\prime}$ \\
\hline 2. & $\begin{array}{l}\text { Asymptotic equator } \\
\text { bundle of } H M^{\prime}\end{array}$ & $S(T(M))$ & $\partial S^{+} H M^{\prime}$ \\
\hline 3. & $\begin{array}{l}\text { Twisted square } \\
\text { of } S(T(M))\end{array}$ & $C(T(M))$ & $\bar{F} H M^{\prime}$ \\
\hline 4. & $\begin{array}{l}\text { Core of the top } \\
\text { stratum of } C(T(M))\end{array}$ & $\bar{S}(T(M))$ & $P M$ \\
\hline 5. & $\begin{array}{l}\text { 1-dimensional foliation } \\
\text { of } \bar{S}(M)\end{array}$ & $\overline{\mathscr{G}}$ & $\mathscr{G}$ \\
\hline 6. & $\begin{array}{l}\text { 1-dimensional foliation } \\
\text { of } D(T(M))\end{array}$ & $\mathscr{G}$ & $\overline{\mathscr{G}}$ \\
\hline 7. & $\begin{array}{l}\text { 1-dimensional foliation } \\
\text { of } S(T(M))\end{array}$ & $\mathscr{G}_{S}$ & $\overline{\mathscr{G}}$ \\
\hline
\end{tabular}
of that of $\S \S 1-3$. In this table, $M=H M^{\prime}$, where $M^{\prime}$ is a (connected) locally

TABLE 1. Reconciliation of notation 
symmetric space whose sectional curvatures are all negative. In fact, $M^{\prime}$ is (real) hyperbolic in entries 3,4 , and 5 .

\section{THE TRANSFERRED SURGERY OBSTRUCTION}

In this section, we prove a generalization of the theorem due to C. T. C. Wall [64] that states that surgery obstructions are unchanged by taking products with the complex projective space $C P^{2}$. Let $k$ be an even positive integer, and let $C^{2 k}$ equal the orbit space $S^{k} \times S^{k} / \phi$, where $S^{k}$ is the $k$-dimensional sphere and $\phi: Z_{2} \times\left(S^{k} \times S^{k}\right) \rightarrow S^{k} \times S^{k}$ is the group action defined by $\phi(1,(x, y))=(y, x)$ and $\phi(0,(x, y))=(x, y)$. Note that $C^{4}=C P^{2}$. A special case of the main theorem of this section (see Theorem 4.3) says that surgery obstructions remain unchanged under products with $C^{2 k}$. The fact that $C^{2 k}$ is not a manifold when $k>2$ gives rise to difficulties both in formulating such a product theorem and in proving it.

Notation. $\quad N$ and $M$ denote compact manifolds with $\operatorname{dim}(N)=\operatorname{dim}(M) \geq 5$. The universal covers of $N$ and $M$ come equipped with orientations. Let $h:(N, \partial N) \rightarrow(M, \partial M)$ denote a degree one normal map such that $h: \partial N \rightarrow$ $\partial M$ is a homotopy equivalence. Set $\pi=\pi_{1}(M)$, and let $w_{1}: \pi \rightarrow Z_{2}$ denote the homomorphism induced by the first Steifel-Whitney class for $T(M)$. Let $\sigma(h) \in L_{m}^{h}\left(\pi, w_{1}\right)$ denote the Wall obstruction to completing surgery (up to homotopy equivalence) on $h$ modulo $h \mid \partial N$, where $m=\operatorname{dim}(M)$ (cf. [64]). $\xi^{k+1} \rightarrow M$ denotes a real vector bundle over $M$ that has $R^{k+1}$ for fiber. $D\left(\xi^{k+1}\right)$ is a smooth subbundle of $\xi^{k+1}$ that has the $(k+1)$-dimensional disc as fiber. $S\left(\xi^{k+1}\right)$ is the spherical fiber bundle associated to $D\left(\xi^{k+1}\right)$ (that is, each fiber of $S\left(\xi^{k+1}\right)$ is the boundary of the corresponding disc fiber of $\left.D\left(\xi^{k+1}\right)\right)$. Set $S \times S\left(\xi^{k+1}\right)=\bigcup_{p \in M} S_{p}\left(\xi^{k+1}\right) \times S_{p}\left(\xi^{k+1}\right)$, where $S_{p}\left(\xi^{k+1}\right)$ is the fiber of $S\left(\xi^{k+1}\right) \rightarrow M$ over $p \in M$. Let $\phi: Z_{2} \times S \times S\left(\xi^{k+1}\right) \rightarrow S \times S\left(\xi^{k+1}\right)$ be the group action defined by $\phi(1,(x, y))=(y, x)$ and $\phi(0,(x, y))=(x, y)$. Set $C\left(\xi^{k+1}\right)$ equal to the orbit space $S \times S\left(\xi^{k+1}\right) / \phi$. Let $h^{*} D\left(\xi^{k+1}\right) \rightarrow N$, $h^{*} S\left(\xi^{k+1}\right) \rightarrow N, h^{*} C\left(\xi^{k+1}\right) \rightarrow N$ denote the pullbacks along $h:(N, \partial N) \rightarrow$ $(M, \partial M)$ of the bundles $D\left(\xi^{k+1}\right) \rightarrow M, S\left(\xi^{k+1}\right) \rightarrow M, C\left(\xi^{k+1}\right) \rightarrow M$. Note that the map $h:(N, \partial N) \rightarrow(M, \partial M)$ lifts to canonical maps $D(h): h^{*} D\left(\xi^{k+1}\right)$ $\rightarrow D\left(\xi^{k+1}\right), S(h): h^{*} S\left(\xi^{k+1}\right) \rightarrow S\left(\xi^{k+1}\right), C(h): h^{*} C\left(\xi^{k+1}\right) \rightarrow C\left(\xi^{k+1}\right)$.

4.1. Surgery on $C(h): h^{*} C\left(\xi^{k+1}\right) \rightarrow C\left(\xi^{k+1}\right)$. Note that $C\left(\xi^{k+1}\right)$ is a stratified space having two strata. The lower stratum is just the fixed point set of the action $\phi$. This can be identified with $S\left(\xi^{k+1}\right)$ by equating each $S_{p}\left(\xi^{k+1}\right)$ with the diagonal of each product $S_{p}\left(\xi^{k+1}\right) \times S_{p}\left(\xi^{k+1}\right)$. Thus, the lower stratum of $C\left(\xi^{k+1}\right)$ is just $S\left(\xi^{k+1}\right)$, and the upper stratum equals $C\left(\xi^{k+1}\right)-S\left(\xi^{k+1}\right)$. There is a neighborhood for $S\left(\xi^{k+1}\right)$ in $C\left(\xi^{k+1}\right)$ that is equal to the total space of a fiber bundle $\tau \rightarrow S\left(\xi^{k+1}\right)$ that has cone $\left(R P^{k-1}\right)$ for fiber, where $R P^{k-1}$ 
is $(k-1)$-dimensional real projective space. We also have that $h^{*} C\left(\xi^{k+1}\right)$ is a stratified space having $h^{*} S\left(\xi^{k+1}\right)$ for lower stratum and having $h^{*} C\left(\xi^{k+1}\right)-$ $h^{*} S\left(\xi^{k+1}\right)$ for upper stratum. The bundle $\tau \rightarrow S\left(\xi^{k+1}\right)$ pulls back along the map $S(h): h^{*} S\left(\xi^{k+1}\right) \rightarrow S\left(\xi^{k+1}\right)$ to a bundle denoted $h^{*} \tau \rightarrow h^{*} S\left(\xi^{k+1}\right)$, the total space of which is a neighborhood for $h^{*} S\left(\xi^{k+1}\right)$ in $h^{*} C\left(\xi^{k+1}\right)$. The following properties are satisfied by the maps $C(h): h^{*} C\left(\xi^{k+1}\right) \rightarrow C\left(\xi^{k+1}\right)$ and $S(h): h^{*} S\left(\xi^{k+1}\right) \rightarrow S\left(\xi^{k+1}\right)$.

(a) $S(h):\left(h^{*} S\left(\xi^{k+1}\right), h^{*} S\left(\xi^{k+1}\right)_{\mid \partial N}\right) \rightarrow\left(S\left(\xi^{k+1}\right), S\left(\xi^{k+1}\right)_{\mid \partial M}\right)$ is a degree one normal map of compact manifold pairs such that $S(h): h^{*} S\left(\xi^{k+1}\right)_{\mid \partial N} \rightarrow$ $S\left(\xi^{k+1}\right)_{\mid \partial M}$ is a homotopy equivalence.

(b) $C(h) \mid h^{*} S\left(\xi^{k+1}\right)=S(h)$.

(c) For each $p \in h^{*} S\left(\xi^{k+1}\right)$, we have that the restriction of $C(h)$ to the fiber $h^{*} \tau_{p}$ is a homeomorphism of $h^{*} \tau_{p}$ onto the fiber $\tau_{S(h)(p)}$.

(d) Set $Y=C\left(\xi^{k+1}\right)-\tau^{0}, \partial_{1} Y=\dot{\tau}, \partial_{2} Y=C\left(\xi^{k+1}\right)_{\mid \partial M}-\tau^{0}, \wedge Y=\partial_{1} Y \cap$ $\partial_{2} Y$, where $\tau^{0}$, $\dot{\tau}$ are the topological interior and boundary of $\tau$ in $C\left(\xi^{k+1}\right)$. Set $X=h^{*} C\left(\xi^{k+1}\right)-h^{*} \tau^{0}, \partial_{1} X=h^{*} \dot{\tau}, \partial_{2} X=h^{*} C\left(\xi^{k+1}\right)_{\mid \partial N}-h^{*} \tau^{0}, \wedge X=$ $\partial_{1} X \cap \partial_{2} X$, where $h^{*} \tau^{0}, h^{*} i$ are the topological interior and boundary of $h^{*} \tau$ in $h^{*} C\left(\xi^{k+1}\right)$. Then

$$
C(h):\left(X, \partial_{1} X, \partial_{2} X, \wedge X\right) \rightarrow\left(Y, \partial_{1} Y, \partial_{2} Y, \wedge Y\right)
$$

is a degree one normal map of compact manifold 4-tuples such that

$$
C(h):\left(\partial_{2} X, \wedge X\right) \rightarrow\left(\partial_{2} Y, \wedge Y\right)
$$

is a homotopy equivalence of pairs.

There are two types of surgeries that we allow to be performed on $C(h)$ : $h^{*} C\left(\xi^{k+1}\right) \rightarrow C\left(\xi^{k+1}\right)$. We may do surgery along the lower stratum of $C(h)$ as follows. Choose a framed embedding $S^{j} \times D^{k+m-j} \subset h^{*} S\left(\xi^{k+1}\right)$ that represents an element in the homotopy group kernel of $S(h)$. After varying $C(h)$ through a homotopy, each level of which satisfies $4.1(\mathrm{a})-(\mathrm{d})$, we may assume that the restriction $C(h) \mid S^{j} \times D^{k+m-j}$ is a constant map with value equal to $p \in S\left(\xi^{k+1}\right)$. It follows from 4.1 (c) that a trivialization

$$
h^{*} \tau_{\mid S^{j} \times D^{k+m-j}} \simeq\left(S^{j} \times D^{k+m-j}\right) \times \operatorname{cone}\left(R P^{k-1}\right)
$$

is provided for the bundle $h^{*} \tau$ over $S^{j} \times D^{k+m-j}$ by the restricted map $C(h)$ : $h^{*} \tau_{\mid S^{j} \times D^{k+m-1}} \rightarrow \tau_{p}$. Thus, we have a type of "framed embedding" $S^{j} \times$ $D^{k+m-j} \times \operatorname{cone}\left(R P^{k-1}\right) \subset h^{*} C\left(\xi^{k+1}\right)$ along which we can do surgery by cutting out the interior of $S^{j} \times D^{k+m-j} \times \operatorname{cone}\left(R P^{k-1}\right)$ and gluing

$$
D^{j+1} \times \partial\left(D^{k+m-j} \times \operatorname{cone}\left(R P^{k-1}\right)\right)
$$


to

$$
h^{*} C\left(\xi^{k+1}\right)-\operatorname{Int}\left(S^{j} \times D^{k+m-j} \times \operatorname{cone}\left(R P^{k-1}\right)\right)
$$

along the identification

$$
\partial D^{j+1} \times \partial\left(D^{k+m-j} \times \operatorname{cone}\left(R P^{k-1}\right)\right)=S^{j} \times \partial\left(D^{k+m-j} \times \operatorname{cone}\left(R P^{k-1}\right)\right) .
$$

(Here

$\partial\left(D^{k+m-j} \times \operatorname{cone}\left(R P^{k-1}\right)\right)=\partial D^{k+m-j} \times \operatorname{cone}\left(R P^{k-1}\right) \cup D^{k+m-j} \times \partial \operatorname{cone}\left(R P^{k-1}\right)$, and $\partial \operatorname{cone}\left(R P^{k-1}\right)$ is the base of the cone $\left.\left(R P^{k-1}\right).\right)$ The resulting space is also a stratified space having two strata, the lower stratum being equal to the result of doing surgery to $h^{*} S\left(\xi^{k+1}\right)$ along the framed embedding $S^{j} \times$ $D^{k+m-j} \subset h^{*} S\left(\xi^{k+1}\right)$.

The other type of surgery on $C(h): h^{*} C\left(\xi^{k+1}\right) \rightarrow C\left(\xi^{k+1}\right)$ that we allow is called surgery along the upper stratum of $C(h)$. This consists of performing ordinary framed surgeries on the normal map $C(h):\left(h^{*} C\left(\xi^{k+1}\right)-h^{*} \tau\right) \rightarrow$ $\left(C\left(\xi^{k+1}\right)-\tau\right)($ see $4.1(\mathrm{~d}))$.

Whichever of the two preceding types of surgery we apply to $C(h)$, we shall always perform these surgeries away from $h^{*} C\left(\xi^{k+1}\right)_{\mid \partial N}$. The result of performing a finite sequence of such surgeries to $C(h)$ will be a new map $F: \partial_{+} W \rightarrow$ $C\left(\xi^{k+1}\right)$ together with a stratified normal cobordism $F: W \rightarrow C\left(\xi^{k+1}\right)$ satisfying the following properties.

(e) $W$ is a stratified cobordism (with just two strata) from $\partial_{-} W=h^{*} C\left(\xi^{k+1}\right)$ to $\partial_{+} W$. The lower stratum of $W$, denoted $W_{l}$, has a neighborhood in $W$ that is equal to the total space of fiber bundle $\gamma \rightarrow W_{l}$ such that $\gamma_{\mid \partial_{-} W_{l}}=h^{*} \tau$.

(f) $F\left(W_{l}\right)=S\left(\xi^{k+1}\right)$. Moreover, for any $p \in W_{l}$, the restricted map $F \mid \gamma_{p}$ is a homeomorphism from the fiber $\gamma_{p}$ onto the fiber $\tau_{F(p)}$.

(g) $F:\left(W_{l}, \partial_{2} W_{l}\right) \rightarrow\left(S\left(\xi^{k+1}\right), S\left(\xi^{k+1}\right)_{\mid \partial M}\right)$ is a normal cobordism of $S(h)$ : $\left(h^{*} S\left(\xi^{k+1}\right), h^{*} S\left(\xi^{k+1}\right)_{\mid \partial N}\right) \rightarrow\left(S\left(\xi^{k+1}\right), S\left(\xi^{k+1}\right)_{\mid \partial M}\right)$. Here

$$
\partial_{2} W_{l}=\operatorname{closure}\left(\partial W_{l}-\partial_{-} W_{l}-\partial_{+} W_{l}\right) \text {. }
$$

Moreover, $F:\left(Z, \partial_{1} Z, \partial_{2} Z, \wedge Z\right) \rightarrow\left(Y, \partial_{1} Y, \partial_{2} Y, \wedge Y\right)$ is a normal cobordism of the map $C(h):\left(X, \partial_{1} X, \partial_{2} X, \wedge X\right) \rightarrow\left(Y, \partial_{1} Y, \partial_{2} Y, \wedge Y\right)$ given in 4.1(d). Here $Z=W-\gamma^{0}, \partial_{1} Z=\dot{\gamma}, \partial_{2} Z=\operatorname{closure}\left(\partial Z-\partial_{1} Z-\partial_{-} W-\partial_{+} W\right)$, $\wedge Z=\partial_{1} Z \cap \partial_{2} Z$, where $\gamma^{0}$ is the topological interior of $\gamma$ in $W$ and $\dot{\gamma}$ is the topological boundary of $\gamma$ in $W$.

(h) All of the cobordisms $\partial_{2} W_{l}, \partial_{2} Z, \wedge Z$ are product cobordisms.

4.2. The transferred surgery obstruction. Note that $D(h): h^{*} D\left(\xi^{k+1}\right) \rightarrow$ $D\left(\xi^{k+1}\right)$ is a normal null-cobordism for the normal map $S(h): h^{*} S\left(\xi^{k+1}\right) \rightarrow$ $S\left(\xi^{k+1}\right)$. Thus, by the $\pi-\pi$ theorem of C. T. C. Wall [64], if $k \geq 2$, there exists a completion of surgery for $S(h): h^{*} S\left(\xi^{k+1}\right) \rightarrow S\left(\xi^{k+1}\right)$ that extends to a 
completion of surgery for $D(h): h^{*} D\left(\xi^{k+1}\right) \rightarrow D\left(\xi^{k+1}\right)$, where all of these surgeries are performed away from $h^{*} D\left(\xi^{k+1}\right)_{\mid \partial N}$. That is, there exists a normal cobordism

$$
G:\left(V, \partial_{1} V, \partial_{2} V, \wedge V\right) \rightarrow\left(D\left(\xi^{k+1}\right), S\left(\xi^{k+1}\right), D\left(\xi^{k+1}\right)_{\mid \partial M}, S\left(\xi^{k+1}\right)_{\mid \partial M}\right)
$$

of the normal map $D(h)$ satisfying the following properties.

(a) The normal cobordism $G:\left(\partial_{2} V, \wedge V\right) \rightarrow\left(D\left(\xi^{k+1}\right)_{\mid \partial M}, S\left(\xi^{k+1}\right)_{\mid \partial M}\right)$ is a product cobordism.

(b) $\partial_{-} G=D(h)$, and $\partial_{+} G$ is a homotopy equivalence of 4-tuples.

Choose any stratified normal cobordism $F: W \rightarrow C\left(\xi^{k+1}\right)$ of the stratified normal map $C(h): h^{*} C\left(\xi^{k+1}\right) \rightarrow C\left(\xi^{k+1}\right)$ that satisfies the following properties.

(c) $F$ satisfies $4.1(\mathrm{e})-(\mathrm{h})$.

(d) There exists a normal cobordism $G$ as in $4.2\left(\right.$ a), (b) such that $G \mid \partial_{1} V=$ $F \mid W_{l}$. (Recall that $W_{l}$ denotes the lower stratum of $W$.)

Note that it follows from $4.2(\mathrm{a})-(\mathrm{d})$ that there is a well-defined surgery obstruction $\sigma\left(\partial_{+} F\right) \in L_{m+2 k}^{h}\left(\pi^{\prime}, w_{1}^{\prime}\right)$ to completing surgery on the normal map $\partial_{+} F: \partial_{+} W-\gamma^{0} \rightarrow C\left(\xi^{k+1}\right)-\tau^{0}$ by doing surgeries away from $\partial\left(\partial_{+} W-\gamma^{0}\right)$, where $\gamma$ comes from $4.1(\mathrm{e})$ and $\tau$ comes from $4.1(\mathrm{c}), \pi^{\prime}=\pi_{1}\left(C\left(\xi^{k+1}\right)-\tau^{0}\right)$ and $w_{1}^{\prime}: \pi^{\prime} \rightarrow Z_{2}$ is the homomorphism determined by the first Stiefel-Whitney class of the bundle $T\left(C\left(\xi^{k+1}\right)-\tau^{0}\right)$. The orientation for the universal cover of $C\left(\xi^{k+1}\right)-\tau^{0}$ with respect to which $\sigma\left(\partial_{+} F\right)$ is computed is given by the local formula $O_{1}=O_{2} \times O_{3} \times O_{3}$, where $O_{2}$ is the given orientation on the universal cover of $M$, and $\mathrm{O}_{3}$ is any local orientation for a fiber of the bundle $S\left(\xi^{k+1}\right) \rightarrow M$. Let $T: L_{m+2 k}^{h}\left(\pi^{\prime}, w_{1}^{\prime}\right) \rightarrow L_{m+2 k}^{h}\left(\pi, w_{1}\right)$ denote the map of surgery groups induced by the projection map $p: C\left(\xi^{k+1}\right) \rightarrow M$. That is, $p: C\left(\xi^{k+1}\right)-\tau^{0} \rightarrow M$ induces a homomorphism $p_{*}: \pi^{\prime} \rightarrow \pi$, which in turn induces the homomorphism $T$ (provided $k=$ even). We define the transfer of the surgery obstruction $\sigma(h) \in L_{m}^{h}\left(\pi, w_{1}\right)$, denoted by $\sigma\left(h ; \xi^{k+1}\right)$, by the formula

(e) $\sigma\left(h ; \xi^{k+1}\right)=T\left(\sigma\left(\partial_{+} F\right)\right)$.

We claim that $\sigma\left(h ; \xi^{k+1}\right)$ depends only on $h$ and $\xi^{k+1}$. To see this, we choose another normal cobordism $F^{\prime}: W^{\prime} \rightarrow C\left(\xi^{k+1}\right)$ of the normal map $C(h): h^{*} C\left(\xi^{k+1}\right) \rightarrow C\left(\xi^{k+1}\right)$ also satisfying $4.2(\mathrm{c})$, (d). It will suffice to show that $\sigma\left(\partial_{+} F\right)=\sigma\left(\partial_{+} F^{\prime}\right)$. By identifying $F^{\prime}$ and $F$ along their common domain $h^{*} C\left(\xi^{k+1}\right)$, we obtain a third normal cobordism, denoted by $F^{\prime \prime}: W^{\prime \prime} \rightarrow$ $C\left(\xi^{k+1}\right)$, that satisfies the following:

(f) $\partial_{-} F^{\prime \prime}=\partial_{+} F^{\prime}, \partial_{+} F^{\prime \prime}=\partial_{+} F$.

Note that the lower stratum of $F^{\prime \prime}: W^{\prime \prime} \rightarrow C\left(\xi^{k+1}\right)$ bounds the normal null cobordism $G^{\prime \prime}: V^{\prime \prime} \rightarrow D\left(\xi^{k+1}\right)$, where $G^{\prime \prime}$ is the normal map obtained by identifying the normal maps $G: V \rightarrow D\left(\xi^{k+1}\right), G: V^{\prime} \rightarrow D\left(\xi^{k+1}\right)$, associated to 
$F, F^{\prime}$ prior to $4.2\left(\right.$ a), along their common domain $h^{*} D\left(\xi^{k+1}\right)$. Thus, we may apply Wall's $\pi-\pi$ theorem to the lower stratum of $F^{\prime \prime}$ to obtain (after a finite sequence of surgeries has been performed along the lower stratum of $F^{\prime \prime}$ and away from $\partial_{-} F^{\prime \prime}$ and $\partial_{+} F^{\prime \prime}$ ) another normal cobordism, also denoted by $F^{\prime \prime}: W^{\prime \prime} \rightarrow C\left(\xi^{k+1}\right)$, that satisfies in addition to $4.2(\mathrm{f})$ the following property.

(g) The lower stratum $W_{l}^{\prime \prime}$ of $W^{\prime \prime}$ is an $H$-cobordism of manifold pairs.

Let $\gamma^{\prime \prime} \rightarrow W_{l}^{\prime \prime}$ denote the bundle tubular neighborhood for $W_{l}^{\prime \prime}$ in $W^{\prime \prime}$ obtained by pulling $\tau \rightarrow S\left(\xi^{k+1}\right)$ back along $F^{\prime \prime}: W_{l}^{\prime \prime} \rightarrow S\left(\xi^{k+1}\right)$. Note that it follows from $4.2(\mathrm{f})$, (g) that $F^{\prime \prime}: W^{\prime \prime}-\left(\gamma^{\prime \prime}\right)^{0} \rightarrow C\left(\xi^{k+1}\right)-\tau^{0}$ is a normal cobordism that makes $\sigma\left(\partial_{+} F^{\prime}\right)$ equivalent to $\sigma\left(\partial_{+} F\right)$ in the surgery group $L_{m}\left(\pi^{\prime}, w_{1}^{\prime}\right)$.

This completes the verification that the surgery obstruction transfer element $\sigma\left(h ; \xi^{k+1}\right)$ depends only on $h$ and $\xi^{k+1}$.

We can now state the main result of this section.

Theorem 4.3. Suppose that $k$ is an even positive integer, that $\xi^{k+1}$ is an orientable vector bundle, and that $\operatorname{dim}(M)=0 \bmod 4$ and $\operatorname{dim}(M) \geq 5$. Then $\sigma(h)$ and $\sigma\left(h ; \xi^{k+1}\right)$ are equal when $L_{m}^{h}\left(\pi, w_{1}\right)$ and $L_{m+2 k}^{h}\left(\pi, w_{1}\right)$ are identified under the Wall periodicity isomorphism. Moreover, if $\mathrm{Wh}(\pi \times A)=0$ holds. for all finitely generated free abelian groups $A$, then $\sigma(h)=\sigma\left(h ; \xi^{k+1}\right)$ holds even if $\operatorname{dim}(M) \neq 0 \bmod 4$.

Let $T^{n}$ denote the $n$-torus, $Z^{n}=\pi_{1}\left(T^{n}\right)$, and $g_{n}: L_{m}^{h}\left(\pi, w_{1}\right) \rightarrow$ $L_{m+n}^{h}\left(\pi \times Z^{n}, w_{1}^{\prime}\right)$ denote the homomorphism obtained geometrically by forming products of normal maps (representing elements of $L_{m}^{h}\left(\pi, w_{1}\right)$ ) with the identity map 1: $T^{n} \rightarrow T^{n}$. Let $w_{1}^{\prime}: \pi \times Z^{n} \rightarrow Z_{2}$ denote the composite map $\pi \times Z^{n} \stackrel{\text { proj }}{\rightarrow} \pi \stackrel{w_{1}}{\rightarrow} Z_{2}$. There is the following corollary of Theorem 4.3.

Corollary 4.3. Suppose that $k$ is an even positive integer, that $\xi^{k+1}$ is an orientable vector bundle, and $\operatorname{dim}(M) \geq 5$. (We do not assume that $\operatorname{dim}(M)=$ $0 \bmod 4$, nor do we assume that $\mathrm{Wh}(\pi \times A)=0$ for all relevant $A$.) Let $n$ be a positive integer such that $n+\operatorname{dim}(M)=0 \bmod 4$. Then we have that $g_{n}(\sigma(h))=g_{n}\left(\sigma\left(h ; \xi^{k+1}\right)\right)$ when $L_{m+n}^{h}\left(\pi \times Z^{n}, w_{1}^{\prime}\right)$ and $L_{m+n+2 k}^{h}\left(\pi \times Z^{n}, w_{1}^{\prime}\right)$ are identified by the Wall periodicity isomorphism.

Remark 4.3. There is a more general version of Theorem 4.3 for when $M$ and $N$ are not assumed to be compact manifolds, which we describe in this remark. A continuous map $f: A \rightarrow B$ between topological spaces $A, B$ is said to have compact support if there is a compact subset $X \subset A$ such that the following holds: $f: A-X \rightarrow B-f(X)$ is a homeomorphism. Now if in Theorem 4.3 we drop the hypothesis that $M$ and $N$ are compact, and instead assume that $h:(N, \partial N) \rightarrow(M, \partial M)$ is a degree one normal map having compact support such that $h: \partial N \rightarrow \partial M$ is a homotopy equivalence, then the conclusions of 
Theorem 4.3 still remain true. The proof given for Theorem 4.3 below also works for this more general version of 4.3. There is also a similar generalization of Corollary 4.3. For future reference we make the following definition. A homotopy $f_{t}: A \rightarrow B, t \in[0,1]$, is said to have compact support if there is a compact subset $X \subset A$ such that for each $t \in[0,1]$ the map $f_{t}: A-X \rightarrow$ $B-f_{t}(X)$ is the same homeomorphism.

Proof of Theorem 4.3. We first remark that if $h:(N, \partial N) \rightarrow(M, \partial M)$ is normally cobordant $\bmod h \mid \partial N$ to $h^{\prime}:\left(N^{\prime}, \partial N^{\prime}\right) \rightarrow(M, \partial M)$ then we must have that $\sigma\left(h ; \xi^{k+1}\right)=\sigma\left(h^{\prime} ; \xi^{k+1}\right)$. The verification of this again involves Wall's $\pi-\pi$ theorem and is similar to the argument given directly preceding the statement of Theorem 4.3. The details are left to the reader.

Until further notice we assume that $\operatorname{dim}(M)=0 \bmod 4$.

We claim that $h:(N, \partial N) \rightarrow(M, \partial M)$ is normally cobordant $\bmod h \mid \partial N$ to a normal map $h^{\prime}:\left(N^{\prime}, \partial N^{\prime}\right) \rightarrow(M, \partial M)$ which satisfies the following properties.

4.4. (a) There is a handle body $A \subset M-\partial M$ that has one zero-handle, a finite number of one-handles, and no other handles.

(b) The inclusion induced map $\pi_{1}(A) \rightarrow \pi_{1}(M)$ is surjective; $h^{\prime}: N^{\prime} \rightarrow M$ induces an isomorphism $\pi_{1}\left(N^{\prime}\right) \simeq \pi_{1}(M)$.

(c) $h^{\prime}: N^{\prime} \rightarrow M$ is in transverse positive to $\partial A$. Set $B=\left(h^{\prime}\right)^{-1}(A), \partial B=$ $\left(h^{\prime}\right)^{-1}(\partial A)$.

(d) $h^{\prime}: N^{\prime}-B \rightarrow M-A$ is a homotopy equivalence; $h^{\prime}: \partial B \rightarrow \partial A$ is a $Z(\pi)$-homology equivalence, where $\pi=\pi_{1}(M)$.

(e) Let $K_{*}\left(h^{\prime} \mid B\right)$ denote the $Z(\pi)$-homology kernel groups for the map $h^{\prime}: B \rightarrow A$. Then $K_{i}\left(h^{\prime} \mid B\right)=0$ if $i \neq m / 2$ and $K_{m / 2}\left(h^{\prime} \mid B\right)$ is a finitely generated free $Z(\pi)$-module.

(f) There is a $Z(\pi)$-basis for $K_{m / 2}\left(h^{\prime} \mid B\right)$ that can be represented by framed embedded spheres $\left(S^{m / 2} \times D^{m / 2}\right)_{j} \subset B, j=1,2, \ldots, x$.

To verify 4.4, we follow Wall [64]. First complete surgery on $h:(N, \partial N) \rightarrow$ $(M, \partial M)$, away from $h \mid \partial N$, up to the middle dimension to get $h^{\prime}:\left(N^{\prime}, \partial N^{\prime}\right) \rightarrow$ $(M, \partial M)$. Choose $A \subset M-\partial M$ satisfying 4.4(a), (b), put $h^{\prime}: N^{\prime} \rightarrow M$ in transverse position to the cores of the handles of $A$, and complete surgery in $N^{\prime}$ on the preimages of these cores. At this point, 4.4(a)-(c) are satisfied, $h^{\prime}: B \rightarrow A$ is a homeomorphism, and $h^{\prime}: N-B \rightarrow M-A$ is $m / 2$-connected. After stabilizing $K_{m / 2}\left(h^{\prime} \mid N^{\prime}-B\right)$ by adding a standard plane to it (cf. [64, p. 47]), we may assume that there is a $Z(\pi)$-basis for $K_{m / 2}\left(h^{\prime} \mid N^{\prime}-B\right)$ represented by framed embeddings $\left(S^{m / 2} \times D^{m / 2}\right)_{j} \subset N^{\prime}-B, j=1,2, \ldots, x$. Note that by adding a finite number of $m / 2$-handles to $B$-one $m / 2$-handle for each $\left(S^{m / 2} \times D^{m / 2}\right)_{j}$-we obtain a larger subset of $N^{\prime}$ (also denoted by $B$ ) into which each $\left(S^{m / 2} \times D^{m / 2}\right)_{j}$ can be isotoped. Then after homotopying $h^{\prime}$ somewhat, we will have completed the verification of 4.4. 
We shall verify the equality $\sigma\left(h^{\prime}\right)=\sigma\left(h^{\prime} ; \xi^{k+1}\right)$ by a direct computation, which is carried out in the following four steps. By the remark made at the beginning of the proof, this will complete the proof of Theorem 4.3 when $\operatorname{dim}(M)=0 \bmod 4$.

Step I. Since $\xi^{k+1}$ is orientable, it follows from 4.4(a) that $\xi^{k+1}$ is trivial over $A$. Thus, by $4.4(\mathrm{c})$, we may obtain the restrictions of $S\left(h^{\prime}\right)$ and $C\left(h^{\prime}\right)$ to the subbundles $\left(h^{\prime}\right)^{*} S\left(\xi^{k+1}\right)_{\mid B}$ and $\left(h^{\prime}\right)^{*} C\left(\xi^{k+1}\right)_{\mid B}$ by forming the products $\left(h^{\prime} \mid B\right) \times I_{1}: B \times S^{k} \rightarrow A \times S^{k}$ and $\left(h^{\prime} \mid B\right) \times I_{2}: B \times C^{2 k} \rightarrow A \times C^{2 k}$. Here $I_{1}: S^{k} \rightarrow S^{k}$ is the identity map on the $k$-sphere, and $I_{2}: C^{2 k} \rightarrow C^{2 k}$ is the identity map on the fiber $C^{2 k}$ of the bundle $C\left(\xi^{k+1}\right) \rightarrow M$.

Since the $Z(\pi)$-homological kernels for all the maps $h^{\prime}, S\left(h^{\prime}\right)$, and $C\left(h^{\prime}\right)$ are concentrated over $B$ (see 4.4(d), (e)), we can use these product structures together with the Kunneth formula to compute the $Z(\pi)$-homological kernels $K_{*}\left(S\left(h^{\prime}\right)\right)$ and $K_{*}\left(C\left(h^{\prime}\right)\right)$.

$$
\text { (a) } K_{*}\left(S\left(h^{\prime}\right)\right)=K_{m / 2}\left(h^{\prime} \mid B\right) \otimes H_{*}\left(S^{k}\right) \text {. }
$$

(b) $K_{*}\left(C\left(h^{\prime}\right)\right)=K_{m / 2}\left(h^{\prime} \mid B\right) \otimes H_{*}\left(C^{2 k}\right)$.

In 4.5 , the tensor product is taken over the integers, while the $Z(\pi)$-module structures come from letting $K_{m / 2}\left(h^{\prime} \mid B\right) \times \pi \rightarrow K_{m / 2}\left(h^{\prime} \mid B\right)$ be the given $\pi$ action and letting $H_{*}\left(S^{k}\right) \times \pi \rightarrow H_{*}\left(S^{k}\right)$ and $H_{*}\left(C^{2 k}\right) \times \pi \rightarrow H_{*}\left(C^{2 k}\right)$ be the trivial $\pi$-actions. The isomorphisms of 4.5 are then isomorphism of right $Z(\pi)$-modules.

Use 4.4(e), (f) and 4.5(a) to choose a finite set of disjoint framed embedded $m / 2$-spheres $\left(S^{m / 2} \times D^{m / 2+k}\right)_{i} \subset(B-\partial B) \times S^{k}, i=1,2, \ldots, x$, which represent a $Z(\pi)$-basis for $K_{m / 2}\left(S\left(h^{\prime}\right)\right)$. Perform surgeries on $C\left(h^{\prime}\right):\left(h^{\prime}\right)^{*} C\left(\xi^{k+1}\right)$ $\rightarrow C\left(\xi^{k+1}\right)$ along these framed $m / 2$-spheres in the lower stratum of $C\left(h^{\prime}\right)$. Let $F: W \rightarrow C\left(\xi^{k+1}\right)$ denote the resulting normal cobordism of $C\left(h^{\prime}\right)$. Let $F$ : $W_{A} \rightarrow A \times C^{2 k}$ denote the resulting normal cobordism of $\left(h^{\prime} \mid B\right) \times I_{2}: B \times C^{2 k} \rightarrow$ $A \times C^{2 k}$. (Note that $W_{A} \subset W$.)

It is left to the reader to verify that $F: W \rightarrow C\left(\xi^{k+1}\right)$ is one of the special normal cobordisms of $C\left(h^{\prime}\right)$ that satisfies $4.2(\mathrm{c}),(\mathrm{d})$ (when we replace $h:(N, \partial N) \rightarrow(M, \partial M)$ in 4.2 by $h^{\prime}:\left(N^{\prime}, \partial N^{\prime}\right) \rightarrow(M, \partial M)$ of 4.4). In particular, we have that $\partial_{+} F: \partial_{+} W \rightarrow C\left(\xi^{k+1}\right)$ satisfies the following properties.

4.6. (a) $\partial_{+} F: \partial_{+} W_{l} \rightarrow S\left(\xi^{k+1}\right)$ is a homotopy equivalence, where $W_{l}$ denotes the lower stratum of $W$.

(b) $\sigma\left(h^{\prime} ; \xi^{k+1}\right)$ equals the image in $L_{m+2 k}\left(\pi, w_{1}\right)$ of the obstruction to completing surgery on $\partial_{+} F:\left(\partial_{+} W-\gamma^{0}\right) \rightarrow\left(C\left(\xi^{k+1}\right)-\tau^{0}\right)$ away from $\partial\left(\partial_{+} W-\gamma^{0}\right)$. (See 4.1 for $\tau^{0}$ and $\gamma^{0}$.)

This completes Step I. 
Step II. In this step, we compute the $Z(\pi)$-homology kernel $K_{*}\left(\partial_{+} F\right)$ to be the following.

\section{7. $K_{*}\left(\partial_{+} F\right)=K_{m / 2}\left(h^{\prime} \mid B\right) \otimes H_{k}\left(C^{2 k}\right)$.}

As a first step in the verification of 4.7 , we compute the homology group $H_{*}\left(C^{2 k}\right)$. Recall that $C^{2 k}=S^{k} \times S^{k} / \sim$, where $(x, y) \sim\left(x^{\prime}, y^{\prime}\right)$ if and only if either $x=x^{\prime}, y=y^{\prime}$ or $x=y^{\prime}, y=x^{\prime}$. Let $q: S^{k} \times S^{k} \rightarrow C^{2 k}$ denote the quotient map, and let $e^{0} \in S^{k}$ be a base point of $S^{k}$. Let $X$ denote the space obtained from $C^{2 k}$ by identifying $q\left(S^{k} \times e^{0}\right)$ to a point. Note that $q: S^{k} \times e^{0} \rightarrow C^{2 k}$ is an embedding and that $X$ is homeomorphic to the $(k+1)$ th suspension $\sum^{k+1}\left(R P^{k-1}\right)$ of $(k-1)$-dimensional real projective space. Thus, using the isomorphism $\bar{H}_{*}(X)=H_{*}\left(C^{2 k}, q\left(S^{k} \times e^{0}\right)\right)$ and the long exact sequence for the pair $\left(C^{2 k}, q\left(S^{k} \times e^{0}\right)\right)$, we compute the homology group $H_{*}\left(C^{2 k}\right)$ to be the following.

4.8. (a)

$$
H_{i}\left(C^{2 k}\right)= \begin{cases}Z, & \text { if } i=0, k, 2 k \\ Z_{2}, & \text { if } i=k+2, k+4, k+6, \ldots, 2 k-2, \\ 0, & \text { otherwise. }\end{cases}
$$

(b) The restricted quotient map $q: S^{k} \times e^{0} \rightarrow C^{2 k}$ induces an isomorphism $H_{i}\left(S^{k} \times e^{0}\right) \cong H_{i}\left(C^{2 k}\right)$ for all $i=0,1, \ldots, k$.

It follows from 4.5, 4.8, and the construction of $F: W \rightarrow C\left(\xi^{k+1}\right)$ given in Step I that the $Z(\pi)$-homology kernels $K_{*}\left(\partial_{-} F\right)$ and $K_{*}(F)$ are related as follows.

$$
K_{i}\left(\partial_{-} F\right)= \begin{cases}K_{m / 2}\left(h^{\prime} \mid B\right) \otimes Z, & \text { if } i=m / 2,(m / 2)+k,(m / 2)+2 k, \\ K_{m / 2}\left(h^{\prime} \mid B\right) \otimes Z_{2}, & \text { if } i=(m / 2)+k+2,(m / 2)+k+4, \\ & (m / 2)+k+6, \cdots,(m / 2)+2 k-2, \\ 0, & \text { otherwise }\end{cases}
$$

In 4.9, the tensor products are taken over the integers, while the $Z(\pi)$-module structures come from letting $K_{m / 2}\left(h^{\prime} \mid B\right) \times \pi \rightarrow K_{m / 2}\left(h^{\prime} \mid B\right)$ be the given $\pi$ action and letting both $Z \times \pi \rightarrow Z$ and $Z_{2} \times \pi \rightarrow Z_{2}$ be the trivial $\pi$-actions. The isomorphisms of 4.9 are then isomorphisms of right $Z(\pi)$-modules.

The $Z(\pi)$-homology kernel $K_{*}\left(F, \partial_{+} F\right)$ can be computed as follows. Let $X$ denotes the space obtained from $W$ by collapsing all of $\partial_{+} W$ to a point. Note that $X$ is homotopy equivalent to the wedge of $x$ distinct copies of the $(m / 2+k+1)$-fold suspension $\sum^{m / 2+k+1}\left(R P^{k-1}\right)$ of $(k-1)$-dimensional real projective space. (Here $x$ is the number of surgeries performed along the lower stratum of $C\left(h^{\prime}\right)$ in Step I.) Since $\bar{H}_{*}(X) \otimes Z(\pi)=K_{*}\left(F, \partial_{+} F\right)$, we have the following computation for $K_{*}\left(F, \partial_{+} F\right)$. 
4.10.

$$
K_{i}\left(F, \partial_{+} F\right)= \begin{cases}(Z(\pi))^{x}, & \text { if } i=(m / 2)+2 k \\ \left(Z_{2}(\pi)\right)^{x}, & \text { if } i=(m / 2)+k+2,(m / 2)+k+4, \\ & (m / 2)+k+6, \ldots,(m / 2)+2 k-2, \\ 0, & \text { otherwise }\end{cases}
$$

We consider now the long exact sequence for the $Z(\pi)$-homology kernels of the pair $\left(F, \partial_{+} F\right): \cdots \rightarrow K_{i+1}\left(F, \partial_{+} F\right) \stackrel{\partial}{\rightarrow} K_{i}\left(\partial_{+} F\right) \rightarrow K_{i}(F) \rightarrow \cdots$. If $i<(m / 2)+k$, then (by 4.9 and 4.10$)$ we have that $K_{i+1}^{+}\left(F, \partial_{+} F\right)=K_{i}(F)=0$, so by exactness of the above sequence, we have the following.

4.11. (a) $K_{i}\left(\partial_{+} F\right)=0$ for $i<(m / 2)+k$.

Since $\partial_{+} F: \partial_{+} W_{l} \rightarrow S\left(\xi^{k+1}\right)$ is a homotopy equivalence (see Step I), it follows that $K_{*}\left(\partial_{+} F\right)$ is dually paired to the cohomology cokernel $K^{*}\left(\partial_{+} F\right)$. Thus, by applying a duality argument and the universal coefficient theorem (which connects the homology kernel and the cohomology cokernel), we deduce from 4.11(a) that the following holds.

(b) $K_{i}\left(\partial_{+} F\right)=0$ if $i>(m / 2)+k$.

Consider the exact sequence

$$
K_{m / 2+k+1}\left(F, \partial_{+} F\right) \stackrel{\partial}{\rightarrow} K_{m / 2+k}\left(\partial_{+} F\right) \rightarrow K_{m / 2+k}(F) \rightarrow K_{m / 2+k}\left(F, \partial_{+} F\right) .
$$

By 4.10 , we have $K_{m / 2+k+i}\left(F, \partial_{+} F\right)=0$ for $i=0,1$, so by exactness of the sequence, we have the following satisfied.

(c) $K_{m / 2+k}\left(\partial_{+} F\right)=K_{m / 2+k}(F)$.

Finally, 4.7 follows from $4.11,4.9$, and $4.8(a)$.

This completes Step II.

Step III. Let $\lambda_{N^{\prime}}: K_{m / 2}\left(h^{\prime}\right) \times K_{m / 2}\left(h^{\prime}\right) \rightarrow Z(\pi)$ and $\lambda_{\partial_{+} W}: K_{m / 2+k}\left(\partial_{+} F\right) \times$ $K_{m / 2+k}\left(\partial_{+} F\right) \rightarrow Z(\pi)$ be the $Z(\pi)$-intersection pairings. Let $\eta \in H_{k}\left(C^{2 k}\right)$ be a chosen generator for $H_{k}\left(C^{2 k}\right)=Z$ (see 4.8), and let $r: K_{m / 2}\left(h^{\prime}\right) \rightarrow$ $K_{m / 2+k}\left(\partial_{+} F\right)$ be the isomorphism of $Z(\pi)$-modules defined by $r(\alpha)=\alpha \otimes \eta$ (see 4.7 and 4.4). The purpose of this step is to verify the following claim.

Claim 4.12. For any $\alpha, \beta \in K_{m / 2}\left(h^{\prime}\right)$, we have that

$$
\lambda_{N^{\prime}}(\alpha, \beta)=\lambda_{\partial_{+} W}(r(\alpha), r(\beta)) .
$$

To verify Claim 4.12 , we first need a geometric description of the isomorphism $r: K_{m / 2}\left(h^{\prime}\right) \rightarrow K_{m / 2+k}\left(\partial_{+} F\right)$. Let $\left(S^{m / 2} \times D^{m / 2}\right)_{j} \subset B, j=1,2, \ldots, x$, be the framed embeddings given in 4.4(f). Choose $x$ distinct points $p_{1}, p_{2}$, $\ldots, p_{x}$ in the lower stratum $S^{k}$ of $C^{2 k}$. Choose the framed embeddings $\left(S^{m / 2} \times D^{m / 2+k}\right)_{j} \subset B \times S^{k}, j=1,2, \ldots, x$, along which surgery is done in Step I to just be thickenings of the embeddings $\left(S^{m / 2} \times D^{m / 2}\right) \times p_{j} \subset B \times S^{k}$, $j=1,2, \ldots, x$. Let $q: S^{k} \times S^{k} \rightarrow C^{2 k}$ be the quotient map, and choose 
points $\left(a_{j}, a_{j}\right) \in S^{k} \times S^{k}, j=1,2, \ldots, x$, such that $q\left(a_{j}, a_{j}\right)=p_{j}$. Consider the embedded manifolds $T_{j} \subset B \times C^{2 k}, j=1,2, \ldots, x$, where $T_{j}=$ $\left(S^{m / 2} \times 0\right)_{j} \times q\left(S^{k} \times a_{j}\right)$. Note that the association $\left(S^{m / 2} \times 0\right)_{j} \leftrightarrow T_{j}$ is a geometric realization of the isomorphism $K_{m / 2}\left(h^{\prime}\right) \cong K_{m / 2+k}\left(\partial_{-} F\right)$ given in $4.5(b)$ and $4.8(b)$.

The effect on each embedding $T_{i} \subset \partial_{-} W$ of performing surgeries on $\partial_{-} F$ along the framed embeddings $\left(S^{m / 2} \times D^{m / 2+k}\right)_{j} \subset B \times S^{k}, j=1,2, \ldots, x$, in the lower stratum of $\partial_{-} W$ is to change each $T_{i} \subset \partial_{-} W$ to an embedding $S_{i} \subset \partial_{+} W-\partial_{+} W_{l}$, where $S_{i}$ is an $(m / 2+k)$-dimensional sphere and $\partial_{+} W_{l}$ denotes the lower stratum of $\partial_{+} W$. To see this, we must recall the surgery process for surgery along the lower stratum of $\partial_{-} F$ discussed in 4.1. First the framed embeddings $\left(S^{m / 2} \times D^{m / 2+k}\right)_{j} \subset \partial_{-} W_{l}$ must be extended to larger "framed embeddings" $\left(S^{m / 2} \times D^{m / 2+k} \times \operatorname{cone}\left(R P^{k-1}\right)\right)_{j} \subset \partial_{-} W$. We can choose these larger "framed embeddings" so as to satisfy the following properties.

(a) For each $j$, there is an embedded $k$-dimensional ball $D_{j} \subset D^{m / 2+k} \times$ $\operatorname{cone}\left(R P^{k-1}\right)$ such that $D_{j} \cap \partial\left(D^{m / 2+k} \times \operatorname{cone}\left(R P^{k-1}\right)\right)=\partial D_{j}$. (See 4.1 for $\partial\left(D^{m / 2+k} \times \operatorname{cone}\left(R P^{k-1}\right)\right)$. $)$

(b) For each $j$, we have that $T_{j} \cap\left(S^{m / 2} \times D^{m / 2+k} \times \operatorname{cone}\left(R P^{k-1}\right)\right)_{j}=$ $\left(S^{m / 2} \times D_{j}\right)_{j}$, and if $j \neq i$, then $T_{j} \cap\left(S^{m / 2} \times D^{m / 2+k} \times \operatorname{cone}\left(R P^{k-1}\right)\right)_{j}=\varnothing$.

(c) In terms of the definition of $T_{j}$ (as $T_{j}=\left(S^{m / 2} \times 0\right)_{j} \times q\left(S^{k} \times a_{j}\right)$ ), the ball $D_{j}$ is just a tubular neighborhood for $q\left(a_{j}, a_{j}\right)$ in $q\left(S^{k} \times a_{j}\right)$ and the product $\left(S^{m / 2} \times D_{j}\right)_{j}$ is the same as $\left(S^{m / 2} \times 0\right)_{j} \times D_{j}$.

To perform surgery on $\partial_{-} F$ along the "framed embedding"

$$
\left(S^{m / 2} \times D^{m / 2+k} \times \operatorname{cone}\left(R P^{k-1}\right)\right)_{j} \subset \partial_{-} W,
$$

we cut out its interior and glue in its place

$$
\left(D^{m / 2+1} \times \partial\left(D^{m / 2+k} \times \operatorname{cone}\left(R P^{k-1}\right)\right)\right)_{j} .
$$

This has the effect on $T_{j}$ of cutting out the interior of $\left(S^{m / 2} \times D_{j}\right)_{j}$ and gluing $\left(D^{m / 2+1} \times \partial D_{j}\right)_{j}$ in its place (see (a)-(c) above). That is,

$$
S_{j}=\overline{\left(T_{j}-\left(S^{m / 2} \times D_{j}\right)_{j}\right)} \cup\left(D^{m / 2+1} \times \partial D_{j}\right)_{j} .
$$

It follows from (a) above that $S_{j}$ is embedded in $\partial_{+} W-\partial_{+} W_{l}$. It follows from (b) and (c) above that $S_{j}$ is an $(m / 2+k)$-dimensional sphere.

Note that the association $\left(S^{m / 2} \times 0\right)_{j} \leftrightarrow S_{j}$ is a geometric realization of the isomorphism $r: K_{m / 2}\left(h^{\prime}\right) \rightarrow K_{m / 2+k}\left(\partial_{+} F\right)$. Thus, to verify Claim 4.12, it will suffice to show that the $Z(\pi)$-intersection number for any $\left(S^{m / 2} \times 0\right)_{i}$ and $\left(S^{m / 2} \times 0\right)_{j}$ is equal to the $Z(\pi)$-intersection number of $S_{i}$ and $S_{j}$. (To 
compute the $Z(\pi)$-intersection number of $S_{i}$ and $S_{j}$, we first compute the $Z\left(\pi^{\prime}\right)$-intersection number, where $\pi^{\prime}=\pi_{1}\left(\partial_{+} W-\partial_{+} W_{l}\right)$, and then project this intersection number into $Z(\pi)$.) In verifying this equality of $Z(\pi)$-intersection numbers, we will first show that there is a set isomorphism $f_{i, j}:\left(S^{m / 2} \times 0\right)_{i} \cap$ $\left(S^{m / 2} \times 0\right)_{j} \rightarrow S_{i} \cap S_{j}$. There will be no loss of generality in assuming that the $\left(S^{m / 2} \times 0\right)_{j}, j=1,2, \ldots, x$, are in general positive to one another in $B \times 0$. It follows then that the $S_{j}, j=1,2, \ldots, x$, are also in general position to one another in $\partial_{+} W-\partial_{+} W_{l}$. Note that there are set isomorphisms $g_{i, j}$ : $\left(S^{m / 2} \times 0\right)_{i} \cap\left(S^{m / 2} \times 0\right)_{j} \rightarrow T_{i} \cap T_{j}$ defined by $g_{i, j}(p)=(p) \times q\left(a_{i}, a_{j}\right)$. If when performing surgeries on the "framed embeddings"

$$
\left(S^{m / 2} \times D^{m / 2+k} \times \operatorname{cone}\left(R P^{k-1}\right)\right)_{j} \subset \partial_{-} W, \quad j=1,2, \ldots, x,
$$

we choose the $\left(S^{m / 2} \times D^{m / 2+k} \times \operatorname{cone}\left(R P^{k-1}\right)\right)_{j}$ to be sufficiently small neighborhoods of their cores $\left(S^{m / 2} \times 0\right)_{j}$, then the sets $T_{i} \cap T_{j}$ and $S_{i} \cap S_{j}$ will be equal whenever $i \neq j$. So we may set $f_{i, j}=g_{i, j}$. To compute the $Z(\pi)$-intersection number for any pair $\left(S^{m / 2} \times 0\right)_{i},\left(S^{m / 2} \times 0\right)_{j}$-we denote this intersection number by $I_{i, j}$-we choose a base point $z \in B \times 0$ and a path $h_{k}:[0,1] \rightarrow B \times 0$ for each $k=1,2, \ldots, x$, that starts at $z$ and ends at a point of $\left(S^{m / 2} \times 0\right)_{k}$. Then we have

4.13. $I_{i, j}=\sum_{p} \eta(p) \mu(p)$, where the sum runs over all $p \in\left(S^{m / 2} \times 0\right)_{i} \cap$ $\left(S^{m / 2} \times 0\right)_{j}, \eta(p)$ is the sign of the intersection of $\left(S^{m / 2} \times 0\right)_{i}$ and $\left(S^{m / 2} \times 0\right)_{j}$ at $p$, and $\mu(p)$ is represented by the composite path $h_{i} * g_{i} * g_{j} * h_{j}^{-1}$, where $g_{i}:[0,1] \rightarrow\left(S^{m / 2} \times 0\right)_{i}$ starts at $h_{i}(1)$ and ends at $p$ and $g_{j}:[0,1] \rightarrow\left(S^{m / 2} \times\right.$ $0)_{j}$ starts at $p$ and ends at $h_{j}(1)$. To compute the $Z\left(\pi^{\prime}\right)$-intersection number of a pair $S_{i}, S_{j}$, denoted by $J_{i, j}$, we choose a base point $z^{\prime} \in B \times\left(C^{2 k}-S^{k}\right)$ and choose paths $h_{k}^{\prime}:[0,1] \rightarrow B \times\left(C^{2 k}-S^{k}\right)$ for $k=1,2, \ldots, x$ such that $h_{k}^{\prime}(0)=z^{\prime}$ and $h_{k}^{\prime}(1) \in S_{k}$. We also require that $z^{\prime}, h_{k}^{\prime}$ project to $z, h_{k}$ under the first factor projection $B \times C^{2 k} \rightarrow B$. Thus, we have $z^{\prime} \in \partial_{-} W-\partial_{-} W_{l}$ and Image $\left(h_{k}^{\prime}\right) \subset \partial_{-} W-\partial_{-} W_{l}$. There will be no loss of generality in assuming that the surgeries performed on $\partial_{-} W$ to get $\partial_{+} W$ take place away from $z^{\prime}$ and all the $\operatorname{Image}\left(h_{k}^{\prime}\right)$. Thus, we have $z^{\prime} \in \partial_{+} W-\partial_{+} W_{l}$ and $h_{k}^{\prime}:[0,1] \rightarrow$ $\partial_{+} W-\partial_{+} W_{l}$. Then we have

4.14. $J_{i, j}=\sum_{p^{\prime}} \eta\left(p^{\prime}\right) \mu\left(p^{\prime}\right)$, where the sum runs over all $p^{\prime} \in S_{i} \cap S_{j}, \eta\left(p^{\prime}\right)$ is the sign of the intersection of $S_{i}$ with $S_{j}$ at the point $p^{\prime}$, and $\mu\left(p^{\prime}\right) \in \pi^{\prime}$ is represented by the composite path $h_{i}^{\prime} * g_{i}^{\prime} * g_{j}^{\prime} *\left(h_{j}^{\prime}\right)^{-1}$, where $g_{i}^{\prime}:[0,1] \rightarrow$ $S_{i}$ with $g_{i}^{\prime}(0)=h_{i}^{\prime}(1), g_{i}^{\prime}(1)=p^{\prime}$, and $g_{j}^{\prime}:[0,1] \rightarrow S_{j}$ with $g_{j}^{\prime}(0)=p^{\prime}$, $g_{j}^{\prime}(1)=h_{j}^{\prime}(1)$. We let $\bar{J}_{i, j}$ and $\bar{\mu}\left(p^{\prime}\right)$ denote the image of $J_{i, j}$ and $\mu\left(p^{\prime}\right)$ 
under the projection of $Z\left(\pi^{\prime}\right)$ into $Z(\pi)$ (this projection is induced by the bundle projection $\left.C\left(\xi^{k+1}\right) \rightarrow M\right)$. To complete the verification of Claim 4.12, we must show that $\bar{J}_{i, j}=I_{i, j}$. This last equality is a consequence of 4.13, 4.14 , and 4.15 below.

4.15. For all $p \in\left(S^{m / 2} \times 0\right)_{i} \cap\left(S^{m / 2} \times 0\right)_{j}$, we have that

$$
\eta(p)=\eta\left(f_{i, j}(p)\right) \text { and } \mu(p)=\bar{\mu}\left(f_{i, j}(p)\right) .
$$

The remaining details are left to the reader.

This completes Step III.

Step IV. Let $(G, \lambda, \mu),\left(G, \lambda^{\prime}, \mu^{\prime}\right)$ be two special Hermitian forms (cf. [64, p. 47]) that represent the $L$-group elements $\sigma, \sigma^{\prime} \in L_{m}^{h}\left(\pi, w_{1}\right)$. The purpose of this step is to verify the following claim.

Claim 4.16. Suppose that $(G, \lambda)$ and $\left(G^{\prime}, \lambda^{\prime}\right)$ are isomorphic intersection forms (i.e., there is an isomorphism $r: G \rightarrow G$ of right $Z(\pi)$-modules such that $\forall x, y \in G$ we have $\left.\lambda(x, y)=\lambda^{\prime}(r(x), r(y))\right)$. Then if $m=0 \bmod 4$ we have that $\sigma=\sigma^{\prime}$.

To verify 4.16 , it will suffice to show that $(G, \lambda, \mu) \oplus\left(G^{\prime},-\lambda^{\prime},-\mu^{\prime}\right)$ represents 0 in $L_{m}\left(\pi, w_{1}\right)$. Note that $\lambda \oplus\left(-\lambda^{\prime}\right)((x, r(x)),(y, r(y)))=0$ holds for all $x, y \in G$. So $K=\{(x, r(x)): x \in G\}$ is a subkernel for the intersection form $\lambda \oplus\left(-\lambda^{\prime}\right)$. On the other hand, we have that

$$
\lambda \oplus\left(-\lambda^{\prime}\right)((x, r(x)),(x, r(x)))=0 \Leftrightarrow \mu \oplus\left(-\mu^{\prime}\right)(x, r(x))=0,
$$

provided that $m=0 \bmod 4$. So $K$ is also a subkernel for the self-intersection form $\mu \oplus\left(-\mu^{\prime}\right)$.

This completes Step IV.

The proof of Theorem 4.3, in the special case that $\operatorname{dim}(M)=0 \bmod 4$, is now completed (see Claims 4.12 and 4.16).

Note that Corollary 4.3 follows from this special case $(\operatorname{dim}(M)=0 \bmod 4)$ of Theorem 4.3.

To prove Theorem 4.3 when $\operatorname{dim}(M) \neq 0 \bmod 4$, we apply Corollary 4.3 and use the fact that the homomorphisms $g_{n}: L_{m}^{h}\left(\pi, w_{1}\right) \rightarrow L_{m+n}^{h}\left(\pi \times Z^{n}, w_{1}^{\prime}\right)$ given in Corollary 4.3 are injective whenever $\mathrm{Wh}(\pi \times A)=0$ holds for all finitely generated free abelian groups $A$ (cf. [14]).

This completes the proof of Theorem 4.3.

\section{SPLITTING THE TRANSFERRED MAP}

Let $p: E \rightarrow M$ denote a fiber bundle over the real hyperbolic manifold $M$ that has a compact manifold $F$ for fiber. Let $h:(N, \partial N) \rightarrow(E, \partial E)$ denote a homotopy equivalence of manifold pairs that has compact support (see 4.3). In this section, we formulate a splitting result that states that after transferring $h$ to a suitable bundle map $C(h)$, we may split $C(h)$ over a triangulation for $M$ (see Theorem 5.5). The transfer construction for $C(h)$ is similar to that given in $\S 4$. The proof of this splitting result is given in $\S \S 6-8$. 
We begin by giving notation that will be used in $\S \S 5-8$.

Notation 5.1. $M^{\prime}$ denotes a complete real hyperbolic manifold and $M$ denotes the one-dimensional hyperbolic thickening of $M^{\prime}$. Recall that $M$ is the topological product $M^{\prime} \times R$ equipped with the Riemann tensor given in (3.8.4). Let $D(T(M))$ denote the asymptotic northern hemisphere subbundle of $T(M)$ associated to this thickening (see [23, §2]), and let $S(T(M))$ denote its boundary sphere bundle. There is the symmetric product bundle $C(T(M))$ constructed from $S(T(M))$ as in $\S 3$ and 4. Recall that $C(T(M))$ is a stratified space having the two strata $S(T(M)), C(T(M))-S(T(M))$. Denote by $\xi$, $D(\xi), S(\xi), C(\xi)$ and $\xi_{\partial}, D\left(\xi_{\partial}\right), S\left(\xi_{\partial}\right), C\left(\xi_{\partial}\right)$ the pullbacks of the bundles $T(M), D(T(M)), S(T(M)), C(T(M))$ along the maps $p: E \rightarrow M$ and $p: \partial E \rightarrow M$. Note that $C\left(\xi_{\partial}\right), C(\xi)$ are just the symmetric products of $S\left(\xi_{\partial}\right)$, $S(\xi)$, respectively, and are thus stratified spaces having $S\left(\xi_{\partial}\right), S(\xi)$ for lower stratum. In $\S 4$, we discussed liftings of $h:(N, \partial N) \rightarrow(E, \partial E)$ to bundle maps $D(h):\left(h^{*} D(\xi), h^{*} D\left(\xi_{\partial}\right)\right) \rightarrow\left(D(\xi), D\left(\xi_{\partial}\right)\right), C(h):\left(h^{*} C(\xi), h^{*} C\left(\xi_{\partial}\right)\right) \rightarrow$ $\left(C(\xi), C\left(\xi_{\partial}\right)\right)$, where $h^{*} D(\xi), h^{*} D\left(\xi_{\partial}\right), h^{*} C(\xi), h^{*} C\left(\xi_{\partial}\right)$ denote the pullbacks of the bundles $D(\xi), D\left(\xi_{\partial}\right), C(\xi), C\left(\xi_{\partial}\right)$ along the map $h:(N, \partial N) \rightarrow$ $(E, \partial E)$.

$\bar{S}(T(M))$ is the core of the upper stratum $C(T(M))-S(T(M))$. In (3.23), we discussed a bundle projection $\rho_{\tau}: \tau \rightarrow S(T(M))$, and for any numbers $\varepsilon>\delta>0$, we discussed (in the paragraph preceding Lemma 3.14) subsets ${ }_{\varepsilon} \dot{\tau}$, ${ }_{\delta, \varepsilon} \tau$ of $\tau$. We let $\tau^{\prime}, \tau^{\prime \prime}$ denote the pullbacks of the bundle $\tau \rightarrow S(T(M))$ along the composite maps

$$
S(\xi) \stackrel{\text { proj }}{\rightarrow} S(T(M))
$$

and

$$
h^{*} S(\xi) \stackrel{S(h)}{\rightarrow} S(\xi) \stackrel{\text { proj }}{\rightarrow} S(T(M)),
$$

where $h^{*} S(\xi)$ is the pullback of the bundle $S(\xi) \rightarrow E$ along the map $h: N \rightarrow$ $E$. Likewise, the subsets ${ }_{\varepsilon} \dot{\tau},{ }_{\delta, \varepsilon} \tau$ of $\tau$ pull back to subsets ${ }_{\varepsilon} \dot{\tau}^{\prime},{ }_{\delta, \varepsilon} \tau^{\prime}$ and ${ }_{\varepsilon} \dot{\tau}^{\prime \prime}$, $\delta, \tau^{\prime \prime}$ of $\tau^{\prime}$ and $\tau^{\prime \prime}$

Definition 5.2. Mappings $f: h^{*} D(\xi) \rightarrow D(\xi)$ and $g: h^{*} C(\xi) \rightarrow C(\xi)$ are said to be an admissible pair if the following properties hold.

(a) $g\left(h^{*} S(\xi)\right) \subset S(\xi)$. Moreover, there is $\varepsilon$ such that for all $x \in h^{*} S(\xi)$, $\delta \leq \varepsilon$, we have that $g\left({ }_{\delta} \tau_{x}^{\prime \prime}\right) \subset{ }_{\delta} \tau_{f(x)}^{\prime}$. In fact, the restricted map $g:{ }_{\delta} \tau_{x}^{\prime \prime} \rightarrow{ }_{\delta} \tau_{f(x)}^{\prime}$ is a homeomorphism.

(b) $g\left(h^{*} C(\xi)-{ }_{\varepsilon} \tau^{\prime \prime}\right) \subset C(\xi)-{ }_{\varepsilon} \tau^{\prime} ; g\left(h^{*} C\left(\xi_{\partial j}\right)\right) \subset C\left(\xi_{\partial}\right) ; f\left(h^{*} D\left(\xi_{\partial j}\right)\right) \subset$ $D\left(\xi_{\partial}\right) ; f\left(h^{*} S(\xi)\right) \subset S(\xi)$.

Definition 5.3. A pair of homotopies $f_{t}: h^{*} D(\xi) \rightarrow D(\xi), t \in[0,1]$, and $g_{t}: h^{*} C(\xi) \rightarrow C(\xi), t \in[0,1]$, are said to be an admissible homotopy pair if for each $t \in[0,1]$, the maps $f_{t}, g_{t}$ are an admissible pair with $\varepsilon$ in Definition 5.2 independent of $t$. 
Definition 5.4. Let $K$ denote a triangulation for $M$, and let $f: h^{*} D(\xi) \rightarrow$ $D(\xi), g: h^{*} C(\xi) \rightarrow C(\xi)$ denote a pair of admissible maps. We shall say that $f, g$ are split over the subcomplex $L$ of $K$ if the following properties hold.

(a) For each simplex $\Delta \in L$, the mapping of 4-tuples

$$
f:\left(h^{*} D(\xi), h^{*} D\left(\xi_{\partial}\right), h^{*} S(\xi), h^{*} S\left(\xi_{\partial}\right)\right) \rightarrow\left(D(\xi), D\left(\xi_{\partial}\right), S(\xi), S\left(\xi_{\partial}\right)\right)
$$

is in transverse position to each 4-tuple $p^{-1}(\Delta) \cap\left(D(\xi), D\left(\xi_{\partial}\right), S(\xi), S\left(\xi_{\partial}\right)\right)$, where $p: D(\xi) \rightarrow M$ is the bundle projection. Moreover, the map of 4-tuples

$$
\begin{aligned}
f:( & (p \circ f)^{-1}(\Delta) \cap\left(h^{*} D(\xi), h^{*} D\left(\xi_{\partial}\right), h^{*} S(\xi), h^{*} S\left(\xi_{\partial}\right)\right) \\
& \rightarrow p^{-1}(\Delta) \cap\left(D(\xi), D\left(\xi_{\partial}\right), S(\xi), S\left(\xi_{\partial}\right)\right)
\end{aligned}
$$

is a homotopy equivalence of 4-tuples.

(b) For each simplex $\Delta \in L$, the map

$$
g:\left(h^{*} C(\xi)-h^{*} S(\xi), h^{*} C\left(\xi_{\partial}\right)-h^{*} S\left(\xi_{\partial}\right)\right) \rightarrow\left(C(\xi)-S(\xi), C\left(\xi_{\partial}\right)-S\left(\xi_{\partial}\right)\right)
$$

is in transverse position to the submanifold pair

$$
q^{-1}(\Delta) \cap\left(C(\xi)-S(\xi), C\left(\xi_{\partial}\right)-S\left(\xi_{\partial}\right)\right),
$$

where $q: C(\xi) \rightarrow M$ is the bundle projection. Moreover, the restricted map

$$
\begin{aligned}
& g:(q \circ g)^{-1}(\Delta) \cap\left(h^{*} C(\xi)-h^{*} S(\xi), h^{*} C\left(\xi_{\partial}\right)-h^{*} S\left(\xi_{\partial}\right)\right) \\
& \quad \rightarrow q^{-1}(\Delta) \cap\left(C(\xi)-S(\xi), C\left(\xi_{\partial}\right)-S\left(\xi_{\partial}\right)\right)
\end{aligned}
$$

is a homotopy equivalence of pairs.

Theorem 5.5. Let $K$ denote a triangulation for $M$. Suppose that $\operatorname{dim} F \geq 6$ and

$$
\mathrm{Wh}\left(\pi_{1}(Y) \oplus A\right)=0 \quad \text { and } \quad \mathrm{Wh}\left(\pi_{1}(Y) \oplus Z_{2} \oplus A\right)=0 \text {, where } Y=F \text { or } \partial F,
$$

hold for all finitely generated free abelian groups $A$. Then there are homotopies $f_{t}: h^{*} D(\xi) \rightarrow D(\xi), t \in[0,1]$, and $g_{t}: h^{*} C(\xi) \rightarrow C(\xi), t \in[0,1]$, of $D(h)$ and $C(h)$ that satisfy the following properties.

(a) $f_{t}, g_{t}, t \in[0,1]$, are an admissable pair of homotopies (see Definition 5.3).

(b) Both of the homotopies $f_{t}, g_{t}, t \in[0,1]$, have compact support (see Remark 4.3).

(c) The pair of maps $f_{1}, g_{1}$ are split over all of the triangulation $K$.

(d) Suppose that the restricted map $h: \partial N \rightarrow \partial E$ is known to be split over all of $K$. Then the homotopies $f_{t}, g_{t}, t \in[0,1]$, may be choosen so that the restricted homotopies $f_{t}\left|h^{*} D\left(\xi_{j}\right), g_{t}\right| h^{*} C\left(\xi_{\partial}\right), t \in[0,1]$, are both the constant homotopy. 
Remark 5.6. When the hypotheses

$$
\mathrm{Wh}\left(\pi_{1}(Y) \oplus A\right)=0 \quad \text { and } \quad \mathrm{Wh}\left(\pi_{1}(Y) \oplus Z_{2} \oplus A\right)=0
$$

of Theorem 5.5 do not hold, then there is still the following stable version of Theorem 5.5. There is a positive integer $k$ that depends only on $\operatorname{dim}(C(\xi))$. Let $\bar{p}: \bar{E} \rightarrow M$ denote the composition of maps $E \times T^{k} \stackrel{\text { proj }}{\rightarrow} \stackrel{p}{\rightarrow} M$, where $T^{k}$ is the $k$-dimensional torus. Let $\bar{\xi}$ denote the pullback along the map $\bar{E} \stackrel{\text { proj }}{\longrightarrow} E$ of the bundle $\xi$. Let $\bar{h}: \bar{N} \rightarrow \bar{E}$ denote the product map $h \times I_{k}: N \times T^{k} \rightarrow$ $E \times T^{k}$, where $I_{k}: T^{k} \rightarrow T^{k}$ is the identity map. Then there are homotopies $\bar{f}_{t}: \bar{h}^{*} D(\bar{\xi}) \rightarrow D(\bar{\xi}), t \in[0,1]$, and $\bar{g}_{t}: \bar{h}^{*} C(\bar{\xi}) \rightarrow C(\bar{\xi}), t \in[0,1]$, of $D(\bar{h})$ and $C(\bar{h})$ that satisfy the properties of Theorem $5.5(\mathrm{a})$-(d) if we replace by $\bar{f}_{t}$, $\bar{g}_{t}, \bar{h}: \bar{N} \rightarrow \bar{E}$, and $\bar{\xi}$ the $f_{t}, g_{t}, h: N \rightarrow E$, and $\xi$ of Theorem 5.5(a)-(d).

Outline of the proof of Theorem 5.5. The proof of Theorem 5.5 is given in $\S \S 6-8$.

In $\S 6$, we formulate two splitting theorems needed in the proof of Theorem 5.5. The first of these states that homotopy equivalences (having a fiber bundle over a flat Riemannian manifold for range) can always be split over a triangulation for the flat manifold. The second of these splitting results states that if a homotopy equivalence (having a fiber bundle over a foliated smooth manifold for range space) has sufficiently good foliated control, then it can be split over a triangulation of the base space of its range. The proofs of these two splitting theorems are contained in other papers.

In $\S 7$, we compute the foliated control data for the maps $D(h)$ and $C(h)$ with respect to the asymptotic foliations $\mathscr{F}_{C}$ and $\mathscr{F}_{D}$ for $C(T(M))-S(T(M))$ and $D(T(M))$.

In $\S 8$, we complete the proof of Theorem 5.5. We first obtain the desired splitting for $C(h): h^{*} C(\xi) \rightarrow C(\xi)$ over the thick part of $M$ by using the control data of $\S 7$ and the foliated control splitting theorem of $\S 6$. We then extend this splitting to the desired splitting of $C(h)$ over all of $M$ by applying the first splitting theorem of $\S 6$ over neighborhoods of cusps of $M$ and over neighborhoods of short closed geodesics in $M$.

\section{TWO SPLITTING THEOREMS}

Let $p: E \rightarrow M$ denote a fiber bundle over the manifold $M$ having a compact manifold $F$ for fiber. Set $\partial_{1} E=p^{-1}(\partial M), \partial_{2} E=\overline{\partial E-\partial_{1} E}$, and $\wedge E=$ $\partial_{1} E \cap \partial_{2} E$. Note that $\left(E, \partial_{1} E, \partial_{2} E, \wedge E\right)$ is a manifold 4-tuple; that is, $\partial E=$ $\partial_{1} E \cup \partial_{2} E$ and $\wedge E=\partial\left(\partial_{i} E\right)$ for $i=1,2$. Let $h:\left(N, \partial_{1} N, \partial_{2} N, \wedge N\right) \rightarrow$ $\left(E, \partial_{1} E, \partial_{2} E, \wedge E\right)$ denote a continuous map between manifold 4-tuples. In this section, we formulate two results that state (under two different hypotheses) that the map $h$ may be split over a subcomplex of a triangulation for $M$ (see Theorems 6.2 and 6.6). The proofs of these results can be found in $[29,30]$.

Recall that a regular cell structure $C$ is a $C W$ complex such that for every cell $e \in C$ the spherical boundary of $e$ is embedded as a subcomplex of $C$. 
Definition 6.1. Let $K$ denote a regular cell structure for $M$, and let $L \subset$ $K$ be a subcomplex of $K$. We say that the map $h:\left(N, \partial_{1} N, \partial_{2} N, \wedge N\right) \rightarrow$ $\left(E, \partial_{1} E, \partial_{2} E, \wedge E\right)$ is split over the subcomplex $L$ if the following properties hold.

(a) $h:\left(N, \partial_{1} N, \partial_{2} N, \wedge N\right) \rightarrow\left(E, \partial_{1} E, \partial_{2} E, \wedge E\right)$ is in transverse position to each 4-tuple $\left(p^{-1}(\Delta), p^{-1}(\Delta) \cap \partial_{1} E, p^{-1}(\Delta) \cap \partial_{2} E, p^{-1}(\Delta) \cap \wedge E\right)$ with $\Delta \in L$.

(b) For each $\Delta \in L$, the restricted map

$$
\begin{aligned}
& h:\left((p \circ h)^{-1}(\Delta),(p \circ h)^{-1}(\Delta) \cap \partial_{1} N,(p \circ h)^{-1}(\Delta) \cap \partial_{2} N,(p \circ h)^{-1}(\Delta) \cap \wedge E\right) \\
& \quad \rightarrow\left(p^{-1}(\Delta), p^{-1}(\Delta) \cap \partial_{1} E, p^{-1}(\Delta) \cap \partial_{2} E, p^{-1}(\Delta) \cap \wedge E\right)
\end{aligned}
$$

is a homotopy equivalence of 4-tuples.

We can now state our first theorem, which is a generalization of a result due to F. T. Farrell and W.-c. Hsiang [14].

Theorem 6.2. Suppose that $\operatorname{dim}(F) \geq 6$, that $\operatorname{Wh}(\pi \oplus A)=0$ holds for all finitely generated free abelian groups $A$, where $\pi=\pi_{1}(F)$ or $\pi=\pi_{1}(\partial F)$, and that $M$ is the total space of a disc bundle over a compact closed flat Riemannian manifold. Suppose also that $h:\left(N, \partial_{1} N, \partial_{2} N, \wedge N\right) \rightarrow\left(E, \partial_{1} E, \partial_{2} E, \wedge E\right)$ is a homotopy equivalence of 4-tuples such that the restricted map $h:\left(\partial_{1} N, \wedge N\right) \rightarrow\left(\partial_{1} E, \wedge E\right)$ is already split over the regular cell structure $K$ for $M$. Then there is a homotopy $h_{t}:\left(N, \partial_{1} N, \partial_{2} N, \wedge N\right) \rightarrow\left(E, \partial_{1} E, \partial_{2} E, \wedge E\right), t \in[0,1]$, of $h$ that satisfies the following properties.

(a) $h_{t} \mid \partial_{1} N, t \in[0,1]$, is the constant homotopy.

(b) $h_{1}:\left(N, \partial_{1} N, \partial_{2} N, \wedge N\right) \rightarrow\left(E, \partial_{1} E, \partial_{2} E, \wedge E\right)$ is split over all of $K$.

(c) If the restricted map $h:\left(\partial_{2} N, \wedge N\right) \rightarrow\left(\partial_{2} E, \wedge E\right)$ is already split over all of $K$, then we may choose $h_{t}, t \in[0,1]$, so that the restricted homotopy $h_{t} \mid \partial N, t \in[0,1]$, is the constant homotopy.

Remark 6.3. There is the following stable version of Theorem 6.2 when the hypothesis $\operatorname{Wh}(\pi \oplus A)=0$ of Theorem 6.2 does not hold. Let $T^{k}$ denote the $k$-dimensional torus, and let

$$
h^{k}:\left(N^{k}, \partial_{1} N^{k}, \partial_{2} N^{k}, \wedge N^{k}\right) \rightarrow\left(E^{k}, \partial_{1} E^{k}, \partial_{2} E^{k}, \wedge E^{k}\right)
$$

denote the Cartesian product of the map $h$ with the identity map $T^{k} \rightarrow T^{k}$. Note that composite map

$$
E^{k}=E \times T^{k} \stackrel{\text { proj }}{\rightarrow} E \stackrel{p}{\rightarrow} M
$$

defines a bundle projection $p^{k}: E^{k} \rightarrow M$ that has $F^{k}=F \times T^{k}$ for fiber. The stable version of Theorem 6.2 states that there is a positive integer $x$, which depends only on $\operatorname{dim}(M)$, such that if $k \geq x$, then the conclusions of Theorem 6.2 are true for $h^{k}, p^{k}$ (in place of $h, p$ ).

In our second theorem of this section, we assume that $M$ is a Riemannian manifold equipped with a smooth foliation $\mathscr{F}$. If $\partial M \neq \varnothing$, then we assume that $\mathscr{F}$ foliates $\partial M$. That is, if a leaf $L$ of $\mathscr{F}$ intersects $\partial M$, then $L \subset \partial M$, and the collection of all such leaves is a smooth foliation for $\partial M$. 
Our second splitting theorem states roughly that if $h:\left(N, \partial_{1} N, \partial_{2} N, \wedge N\right) \rightarrow$ $\left(E, \partial_{1} E, \partial_{2} E, \wedge E\right)$ is a homotopy equivalence over the subcomplex $L$ of $K$ that has good "control" over $L$ with respect to $\mathscr{F}$, then $h$ can be split over $L$. We need some more notation and a definition before giving a precise formulation of this result.

For any subset $Y \subset M$ and any numbers $\alpha, \delta>0$, we denote by $Y^{\alpha, \delta}$ the subset of all $x \in M$ such that there are paths $f:[0,1] \rightarrow M$ and $g:[0,1] \rightarrow$ $M$ satisfying the following properties.

(a) $f(0) \in Y, f(1)=g(0)$, and $g(1)=x$.

(b) Image $(f)$ lies in a leaf $L$ of $\mathscr{F}$. The diameter of $\operatorname{Image}(f)$ in $L$, computed with respect to the Riemannian structure inherited by $L$ from $M$, is less than $\alpha$.

(c) The diameter of Image $(g)$ in $M$ is less than $\delta$.

Definition 6.4. A map $h:\left(N, \partial_{1} N, \partial_{2} N, \wedge N\right) \rightarrow\left(E, \partial_{1} E, \partial_{2} E, \wedge E\right)$ is said to be $(\alpha, \delta)$-controlled over $(Y, \mathscr{F})$, for some subset $Y \subset M$, if there are maps

$$
\begin{aligned}
g:\left(p^{-1}(Y), p^{-1}(Y) \cap \partial_{1} E, p^{-1}(Y) \cap \partial_{2} E, p^{-1}(Y) \cap \wedge E\right) \\
\quad \rightarrow\left(N, \partial_{1} N, \partial_{2} N, \wedge N\right),
\end{aligned}
$$

$$
\begin{gathered}
H:\left((p \circ h)^{-1}(Y),(p \circ h)^{-1}(Y) \cap \partial_{1} N,(p \circ h)^{-1}(Y) \cap \partial_{2} N,(p \circ h)^{-1}(Y) \cap \wedge N\right) \\
\quad \times[0,1] \rightarrow\left(N, \partial_{1} N, \partial_{2} N, \wedge N\right),
\end{gathered}
$$

and

$$
\begin{aligned}
G: & \left(p^{-1}(Y), p^{-1}(Y) \cap \partial_{1} E, p^{-1}(Y) \cap \partial_{2} E, p^{-1}(E) \cap \wedge E\right) \times[0,1] \\
& \rightarrow\left(E, \partial_{1} E, \partial_{2} E, \wedge E\right)
\end{aligned}
$$

that satisfy the following properties.

(a) $H$ is a homotopy from $g \circ\left(h \mid(p \circ h)^{-1}(Y)\right)$ to the inclusion map $(p \circ h)^{-1}(Y) \subset N$, and $G$ is a homotopy from $h \circ g$ to the inclusion map $p^{-1}(Y) \subset E$.

(b) Let $f:[0,1] \rightarrow M$ denote any of the following composite maps:

$$
\begin{array}{cl}
{[0,1]=(y) \times[0,1] \stackrel{G}{\rightarrow} E \stackrel{p}{\rightarrow} M,} & \text { where } y \in p^{-1}(Y), \\
{[0,1]=(y) \times[0,1] \stackrel{H}{\rightarrow} N \stackrel{h}{\rightarrow} E \stackrel{p}{\rightarrow} M,} & \text { where } y \in(p \circ h)^{-1}(Y) .
\end{array}
$$

Then there is another map $f^{\prime}:[0,1] \rightarrow M$ that satisfies (c) and (d).

(c) $d\left(f(t), f^{\prime}(t)\right) \leq \delta$ holds for all $t \in[0,1]$, where $d($,$) denotes the$ metric on $M$ coming from the Riemannian structure.

(d) Image $\left(f^{\prime}\right)$ is contained in a leaf $L$ of $\mathscr{F}$. Moreover, the diameter of Image $\left(f^{\prime}\right)$ in $L$, computed with respect to the Riemannian structure inherited by $L$ from $M$, is less than or equal to $\alpha$.

Definition 6.5. A homotopy $h_{t}:\left(N, \partial_{1} N, \partial_{2} N, \wedge N\right) \rightarrow\left(E, \partial_{1} E, \partial_{2} E, \wedge E\right)$, $t \in[0,1]$, is said to be $(\alpha, \delta)$-controlled over $(Y, \mathscr{F})$ for some subset $Y \subset M$ if the following properties hold. 
(a) For each $t \in[0,1]$, the map $h_{t}$ is $(\alpha, \delta)$-controlled over $(Y, \mathscr{F})$.

(b) For each $x \in N$, there is a path $p_{x}:[0,1] \rightarrow M$ in $M$ that satisfies (c) and (d).

(c) Image $\left(p_{x}\right)$ is contained in a leaf $L$ of $\mathscr{F}$. Moreover, the diameter of Image $\left(p_{x}\right)$ in $L$, with respect to the Riemann metric inherited by $L$ from $M$, is less than or equal to $\alpha$.

(d) $d\left(p_{x}(t), p \circ h_{t}(x)\right) \leq \delta$ holds for all $t \in[0,1]$.

We shall say that the support of a map

$$
h:\left(N, \partial_{1} N, \partial_{2} N, \wedge N\right) \rightarrow\left(E, \partial_{1} E, \partial_{2} E, \wedge E\right)
$$

lies over a subset $Y \subset M$ if the restricted map $h: N-(p \circ h)^{-1}(Y) \rightarrow E-p^{-1}(Y)$ is a homeomorphism.

We can now state the second splitting theorem of this section. Recall that the definition of "uniform $(\beta, \sigma)$-flatness" for the foliation $\mathscr{F}$ of $M$ was given in Definition 3.2.1. For any subcomplex $L$ of $K$ (where $K$ is a regular cell structure for $M$ ), we let $|L|$ denote the underlying subset of $L$.

Theorem 6.6. Suppose that $\operatorname{dim}(F) \geq 6$ and that $\operatorname{Wh}(\pi \oplus A)=0$ holds for all finitely generated free abelian groups $A$, where $\pi=\pi_{1}(F)$ or $\pi=\pi_{1}(\partial F)$. Then there are numbers $\eta, \sigma \in(0,1)$ that depend only on $\operatorname{dim}(M)$. Given any numbers $\beta, \varepsilon>0$ and given a subcomplex $L$ of $K$, there is a number $\delta>0$. If $\mathscr{F}$ is uniformly $(\beta, \sigma)$-flat on $|L|$, and if $h:\left(N, \partial_{1} N, \partial_{2} N, \wedge N\right) \rightarrow$ $\left(E, \partial_{1} E, \partial_{2} E, \wedge E\right)$ is $(\eta \beta, \delta)$-controlled over $|L|^{\beta, \varepsilon}$, then there is a homotopy $h_{t}:\left(N, \partial_{1} N, \partial_{2} N, \wedge N\right) \rightarrow\left(E, \partial_{1} E, \partial_{2} E, \wedge E\right), t \in[0,1]$, of $h$ that satisfies the following properties.

(a) $h_{1}:\left(N, \partial_{1} N, \partial_{2} N, \wedge N\right) \rightarrow\left(E, \partial_{1} E, \partial_{2} E, \wedge E\right)$ is split over $L$.

(b) The homotopy $h_{t}, t \in[0,1]$ is $(\beta, \varepsilon)$-controlled over $\left(|L|^{\beta, \varepsilon}, \mathscr{F}\right)$.

(c) Suppose that the support of $h$ lies over the subset $Y \subset M$. Then $h_{t} \mid(p \circ h)^{-1}\left(M-(Y \cap|L|)^{\beta, \varepsilon}\right), t \in[0,1]$, is the constant homotopy.

(d) Suppose that the restricted map $h:\left(\partial_{2} N, \wedge N\right) \rightarrow\left(\partial_{2} E, \wedge E\right)$ is already split over all of $L^{\beta, \varepsilon}$, where $L^{\beta, \varepsilon}$ denotes the minimal subcomplex of $K$ that contains $|L|^{\beta, \varepsilon}$, and that each cell of $K$ has diameter less than $\delta$. Then we may assume (in addition to properties (a)-(c)) that $h_{t} \mid \partial_{2} N, t \in[0,1]$, is the constant homotopy.

Remark 6.7. There is the following stable version of Theorem 6.6 when the hypothesis $\mathrm{Wh}(\pi \oplus A)=0$ of Theorem 6.6 is not satisfied. Suppose that $h$ and $\mathscr{F}$ satisfy all the hypotheses of Theorem 6.6 except the vanishing Whitehead group hypothesis. Let

$$
h^{k}:\left(N^{k}, \partial_{1} N^{k}, \partial_{2} N^{k}, \wedge N^{k}\right) \rightarrow\left(E^{k}, \partial_{1} E^{k}, \partial_{2} E^{k}, \wedge E^{k}\right)
$$

be the Cartesian product of the map $h$ with the identity map $T^{k} \rightarrow T^{k}$ on the $k$-dimensional torus. Note that the composite map $E^{k}=E \times T^{k} \stackrel{\text { proj }}{\rightarrow} E \stackrel{p}{\rightarrow} M$ defines a bundle projection $p^{k}: E^{k} \rightarrow M$ that has $F \times T^{k}$ for fiber. There is a 
positive integer $x$, which depends only on $\operatorname{dim}(M)$, such that if $k \geq x$, then the conclusions of Theorem 6.6 are true for $h^{k}, p^{k}$ (in place of $h, p$ ).

\section{THE ASYMPTOTIC TRANSFER}

In this section, we measure the $(\alpha, \delta)$-control of the transferred maps $D(h)$, $C(h)$ with respect to the asymptotic foliations $\mathscr{F}_{D}, \mathscr{F}_{C}$ for $D(T(M))$, $C(T(M))-S(T(M))$. This is accomplished by using an asymptotic transfer construction, similar to those used in [23, 25].

We first state the main result of this section. Then we describe the asymptotic transfer construction. Finally, we use the asymptotic transfer to complete the proof of the main result.

Let $h, D(h), S(h), C(h)$ be the maps from $\S 5$. In the following lemma the foliated control properties for the maps $D(h), S(h)$, and $C(h): h^{*} C(\xi)-$ $h^{*} S(\xi) \rightarrow C(\xi)-S(\xi)$ are computed with respect to the projections $D(\xi) \rightarrow$ $D(T(M)), S(\xi) \rightarrow S(T(M))$, and $C(\xi)-S(\xi) \rightarrow C(T(M))-S(T(M))$, and with respect to the asymptotic foliations of $D(T(M)), S(T(M))$, and $C(T(M))-S(T(M))$. Recall that the Riemannian metrics for $D(T(M))$, $S(T(M))$, and $C(T(M))-S(T(M))$ were described in Lemma 2.1. The notion of $(\alpha, \delta)$-controlled homotopy equivalence was given in Definition 6.4 with respect to foliations of Riemannian manifolds.

Lemma 7.1. There is a number $\Theta>0$ such that $D(h), S(h)$, and $C(h): h^{*} C(\xi)$ $-h^{*} S(\xi) \rightarrow C(\xi)-S(\xi)$ are all $(\boldsymbol{\Theta}, 0)$-controlled over $\left(X_{1}, \mathscr{F}_{D}\right),\left(X_{2}, \mathscr{F}_{S}\right)$, and $\left(X_{3}, \mathscr{F}_{C}\right)$. Here $X_{1}, X_{2}, X_{3}$ are any compact subsets of $D(T(M)), S(T(M))$, $C(T(M))-S(T(M))$, and $\mathscr{F}_{D}, \mathscr{F}_{S}, \mathscr{F}_{C}$ denote the asymptotic foliations of $D(T(M)), S(T(M)), C(T(M))-S(T(M))$.

7.2. The asymptotic transfer. Let $Y(h), Y(h \mid \partial N)$ denote the mapping cylinders for the maps $h: N \rightarrow E, h: \partial N \rightarrow \partial E$; i.e., $Y(h)=N \times[0,1] \cup E / \sim$, $Y(h \mid \partial N)=\partial N \times[0,1] \cup \partial E / \sim$, where $z \sim z^{\prime}$ if and only if either $z=z^{\prime}$ or $z=(x, 1)$ and $z^{\prime} \in E$ with $h(x)=z^{\prime}$. Note that there is a deformation retract $r:(Y(h), Y(h \mid \partial N)) \times[0,1] \rightarrow(Y(h), Y(h \mid \partial N))$ of the pair $(Y(h), Y(h \mid \partial N))$ onto the pair $(N \times 0, \partial N \times 0)$ that satisfies the following property.

(a) There is a compact subset $X \subset N$ such that $r(q(x, s), t)=q(x,(1-t) s)$ holds for all $(x, s) \in(N-X) \times[0,1], t \in[0,1]$. Here $q: N \times[0,1] \cup E \rightarrow Y(h)$ is the quotient map.

Now a homotopy inverse $g:(E, \partial E) \rightarrow(N, \partial N)$ to $h$, and homotopies $H:(N, \partial N) \times[0,1] \rightarrow(N, \partial N), G:(E, \partial E) \times[0,1] \rightarrow(E, \partial E)$ of $g \circ h$, $h \circ g$ to the identity maps $1_{N}, 1_{E}$ can be defined as follows.

(b) $(g(x), 0)=r(x, 1)$ for all $x \in E: H(x, t)=r(q(x, 1-t), 1)$ for all $x \in N, t \in[0,1] ; G(x, t)=\bar{r} \circ r(q(x), 1-t)$, where $\bar{r}: Y(h) \rightarrow E$ is the retract defined by

$$
\bar{r}(z)= \begin{cases}z, & \text { if } z \in E \\ h(x), & \text { if } z=q(x, s) \text { with } s<1\end{cases}
$$


In $\S 4$, we discussed liftings of $h:(N, \partial N) \rightarrow(E, \partial E)$ to bundle maps $D(h)$ : $h^{*} D(\xi) \rightarrow D(\xi), S(h): h^{*} S(\xi) \rightarrow S(\xi), C(h): h^{*} C(\xi) \rightarrow C(\xi)$. In this section, we discuss liftings of the maps $g, H, G$ (of 7.2) to maps $D(g): D(\xi) \rightarrow$ $h^{*} D(\xi), S(g): S(\xi) \rightarrow h^{*} S(\xi) . \quad C(g): C(\xi) \rightarrow h^{*} C(\xi), D(H): h^{*} D(\xi) \times$ $[0,1] \rightarrow h^{*} D(\xi), S(H): h^{*} S(\xi) \times[0,1] \rightarrow h^{*} S(\xi), C(H): h^{*} C(\xi) \times[0,1] \rightarrow$ $h^{*} C(\xi), D(G): D(\xi) \times[0,1] \rightarrow D(\xi), S(G): S(\xi) \times[0,1] \rightarrow S(\xi), C(G): C(\xi)$ $\times[0,1] \rightarrow C(\xi)$. We call the collection of 4-tuples $(D(h), D(g), D(H), D(G))$, $(S(h), S(g), S(H), S(G)),(C(h), C(g), C(H), C(G))$ the asymptotic transfer of $(h, g, H, G)$ because the asymptotic foliations for $D(T(M)), S(T(M))$, $C(T(M))$ are used in their construction.

To construct the asymptotic transfers of $(h, g, H, G)$, we first construct liftings for the deformation retract $r$ of 7.2(a) to deformation retracts

$$
\begin{gathered}
D(r): Y(D(h)) \times[0,1] \rightarrow Y(D(h)), \\
S(r): Y(S(h)) \times[0,1] \rightarrow Y(S(h)), \\
C(r): Y(C(h)) \times[0,1] \rightarrow Y(C(h))
\end{gathered}
$$

of the mapping cylinders $Y(D(h)), Y(S(h)), Y(C(h))$ onto the subsets $h^{*} D(\xi)$, $h^{*} S(\xi), h^{*} C(\xi)$. Then the maps $(D(g), D(H), D(G)),(S(g), S(H), S(G))$, and $(C(g), C(H), C(G))$ can be obtained by applying the recipe of 7.2(b), which yielded $g, H, G$ from $r$, to the deformation retracts $D(r), S(r)$, and $C(r)$. We first construct $D(r)$ as follows. Note that for each leaf $L \in \mathscr{F}_{D}$ of the asymptotic foliation $\mathscr{F}_{D}$ of $D(T(M))$ the composite map $L \subset D(T(M)) \stackrel{\text { proj }}{\longrightarrow}$ $M$, denoted by $\rho_{L}: L \rightarrow M$, is a covering space projection. Let $\rho_{L^{\prime}}: L^{\prime} \rightarrow E$, $\rho_{L^{\prime \prime}}: L^{\prime \prime} \rightarrow N, \rho_{L^{*}}: L^{*} \rightarrow Y(h)$ denote the pullback bundles of $\rho_{L}: L \rightarrow M$ along the maps $p: E \rightarrow M, p \circ h: N \rightarrow M, p \circ \bar{r}: Y(h) \rightarrow M$, where $\bar{r}: Y(h) \rightarrow E$ is the canonical projection of the mapping cylinder $Y(h)$ onto the subset $E$. Note that $L^{*}$ equals the mapping cylinder $Y\left(D(h) \mid L^{\prime \prime}\right)$ for the map $D(h): L^{\prime \prime} \rightarrow L^{\prime}$, and that $\rho_{L^{\prime \prime}}, \rho_{L^{\prime}}$ are equal to the restriction of $\rho_{L}^{*}$ to the two ends of $Y\left(D(h) \mid L^{\prime \prime}\right)$. Note that the collections $\mathscr{F}_{D}^{\prime}=\left\{L^{\prime}: L \in \mathscr{F}_{D}\right\}$, $\mathscr{F}_{D}^{\prime \prime}=\left\{L^{\prime \prime}: L \in \mathscr{F}_{D}\right\}$ constitute topological foliations for the spaces $D(\xi)$ and $h^{*} D(\xi)$. Thus, $Y(D(h))$ is the disjoint union of all the $\left\{Y\left(D(h) \mid L^{\prime \prime}\right): L \in \mathscr{F}_{D}\right\}$. Since $\rho_{L^{*}}: Y\left(D(h) \mid L^{\prime \prime}\right) \rightarrow Y(h)$ is a covering space projection, the homotopy retraction $r: Y(h) \times[0,1] \rightarrow Y(h)$ lifts uniquely to a homotopy retraction $r_{L}: Y\left(D(h) \mid L^{\prime \prime}\right) \times[0,1] \rightarrow Y\left(D(h) \mid L^{\prime \prime}\right)$. Define $D(r)$ to be the disjoint union

(c) $D(r)=\bigcup_{L \in \mathscr{F}_{D}} r_{L}$.

The construction of the homotopy retractions $S(r)$ and $C(r)$ is similar to that given for $D(r)$. The asymptotic foliations $\mathscr{F}_{S}, \mathscr{F}_{c}$ for $S(T(M))$ and $C(T(M))$ pull back along $p: E \rightarrow M, p \circ h: N \rightarrow M$ to give foliations $\mathscr{F}_{S}^{\prime}$, $\mathscr{F}_{C}^{\prime}$ and $\mathscr{F}_{S}^{\prime \prime}, \mathscr{F}_{C}^{\prime \prime}$ for $S(\xi), C(\xi)$ and $h^{*} S(\xi), h^{*} C(\xi)$. For any leaves $L^{\prime \prime} \in \mathscr{F}_{S}^{\prime \prime}, L^{\prime} \in \mathscr{F}_{S}^{\prime}$ (or $L^{\prime \prime} \in \mathscr{F}_{C}^{\prime \prime}, L^{\prime} \in \mathscr{F}_{C}^{\prime}$ ) that satisfy $S(h)\left(L^{\prime \prime}\right)=L^{\prime}$ (or that satisfy $\left.C(h)\left(L^{\prime \prime}\right)=L^{\prime}\right)$, we may use the homotopy lifting property for covering space projections to uniquely lift the homotopy retraction $r: Y(h) \times$ $[0,1] \rightarrow Y(h)$ to a homotopy retraction $r_{L}: Y\left(S(h) \mid L^{\prime \prime}\right) \times[0,1] \rightarrow Y\left(S(h) \mid L^{\prime \prime}\right)$ 
(or to a homotopy retraction $r_{L}: Y\left(C(h) \mid L^{\prime \prime}\right) \times[0,1] \rightarrow Y\left(C(h) \mid L^{\prime \prime}\right)$ ). Now we define $S(r)$ and $C(r)$ to be the disjoint unions

(d) $S(r)=\bigcup_{L \in \mathscr{F}_{S}} r_{L}, C(r)=\bigcup_{L \in \mathscr{F}_{C}} r_{L}$.

This completes the discussion of the asymptotic transfer construction. We can now complete the proof of Lemma 7.1.

Proof of Lemma 7.1. The proof is the same in all three cases, so we will prove Lemma 7.1 only for $D(h)$. We have the foliations $\mathscr{F}_{D}^{\prime \prime}, \mathscr{F}_{D}^{\prime}$ of the spaces $h^{*} D(\xi), D(\xi)$ obtained by pulling back the asymptotic foliation $\mathscr{F}_{D}$ for $D(T(M))$. Note that for any leaf $L \in \mathscr{F}_{D}$, there are corresponding leaves $L^{\prime} \in \mathscr{F}_{D}^{\prime}, L^{\prime \prime} \in \mathscr{F}_{D}^{\prime \prime}$ satisfying the following properties.

7.3. (a)

$$
\begin{gathered}
D(h)\left(L^{\prime \prime}\right)=L^{\prime} ; \quad D(g)\left(L^{\prime}\right)=L^{\prime \prime} \\
D(H)\left(L^{\prime \prime} \times[0,1]\right)=L^{\prime \prime} ; \quad D(G)\left(L^{\prime} \times[0,1]\right)=L^{\prime} ; f\left(L^{\prime}\right)=L,
\end{gathered}
$$

where $f: D(\xi) \rightarrow D(T(M))$ is the bundle projection.

(b) The spaces $N, E$ are covered by the spaces $L^{\prime \prime}, L^{\prime}$, and the maps $h: N \rightarrow E, H: N \times[0,1] \rightarrow N, G: E \times[0,1] \rightarrow E$ are covered by the maps $D(h): L^{\prime \prime} \rightarrow L^{\prime}, D(H): L^{\prime \prime} \times[0,1] \rightarrow L^{\prime \prime}, D(G): L^{\prime} \times[0,1] \rightarrow L^{\prime}$.

Note that it follows from $7.3($ a) that $D(h)$ is 0 -controlled in the directions perpendicular to the leaves of $\mathscr{F}_{D}$.

On the other hand, $h, g, H, G$ all have compact support (see 7.2(a) and (b)). So it follows from 7.3(b), and from the relation between the metrics on $L$ and $M$ given in Lemma 2.1, that there is a positive number $\Theta$ such that $D(h)$ is $\Theta$-controlled in the directions tangent to $\mathscr{F}_{D}$.

This completes the proof of Lemma 7.1.

\section{COMPLETION OF THE PROOF FOR THEOREM 5.5}

Before completing the proof of Theorem 5.5, it is convenient to supplement the notation given in Theorem 5.1 by the following.

8.1. More notation. Recall that $M=M^{\prime} \times R$, where $M^{\prime}$ is a real complete hyperbolic manifold and $M^{\prime} \times R$ is equipped with the metric of (3.8.4). For positive numbers $\varepsilon, \beta>0$, we let $M^{{ }^{\epsilon}}$ denote the set of all points of $M^{\prime}$ at which the radius of injectivity of $M^{\prime}$ is greater than $\varepsilon$, and we let $M_{\varepsilon, \beta}$ denote the set of all points of the subset $M^{\prime^{\varepsilon}} \times R$ that are a distance less than $\beta$ from $M^{\prime^{\varepsilon}} \times 0$ in $M$. Let $D_{\varepsilon, \beta}(T(M)), S_{\varepsilon, \beta}(T(M)), C_{\varepsilon, \beta}(T(M)), \bar{S}_{\varepsilon, \beta}(T(M))$ denote the parts of $D(T(M)), S(T(M)), C(T(M)), \bar{S}(T(M))$ that lie over $M_{\varepsilon, \beta}$.

Set $\gamma=C(T(M))-S(T(M))$. In $\S 3$, we discussed the bundle projection $\rho_{\gamma}: \gamma \rightarrow \bar{S}(T(M))$, and for any $\varepsilon>\delta>0$, we discussed the subsets ${ }_{\varepsilon} \dot{\gamma},{ }_{\varepsilon} \gamma,{ }_{\delta, \varepsilon} \gamma$ of $\gamma$ (see (3.26) and the paragraph preceding Lemma 3.14).

Choose positive numbers $\eta, \sigma$ which satisfy the following property. 
(a) The numbers $\eta, \sigma$ work in Theorem 6.6 for any manifold $M$ of Theorem 6.6 that has dimension less than or equal to our present manifold $\gamma$.

We let $K$ denote the triangulation for $M$ given in Theorem 5.5. We shall need three more triangulations $K_{1}, K_{2}, K_{3}$ of $D(T(M)), \gamma, \bar{S}(T(M))$, respectively. We choose the $K_{i}, i=1,2,3$, so that the following property holds.

(b) The $d\left(K_{i}\right), i=1,2,3$, are arbitrarily small. (Here $d\left(K_{i}\right)$ denotes the least upper bound for the diameters of all simplices $\Delta \in K_{i}$.)

Note that there will be no loss of generality in Theorem 5.5 if we assume that $K$ satisfies the following property.

(c) $d(K)$ is arbitrarily small.

As the proof of Theorem 5.5 proceeds, we shall need to make further assumptions about the interrelations of the $K, K_{i}, i=1,2,3$.

We are now equipped with enough notation to begin the proof of Theorem 5.5.

Proof of Theorem 5.5. We shall make the simplifying assumption in the following proof that the fiber $F$ of $p: E \rightarrow M$ has no boundary. The extra details needed to prove Theorem 5.5 when the fiber has a boundary are left to the reader.

We shall carry out the proof of Theorem 5.5 in the following four steps.

Step I. Let $g^{s}: D(T(M)) \rightarrow D(T(M)), s \in(-\infty,+\infty)$, denote the geodesic flow, and let $\mathscr{G}$ denote the foliation of $D(T(M))$ by the flow lines of the geodesic flow. Let $K_{1, s}$ denote the image of the triangulation $K_{1}$ under $g^{s}$, and let $\rho_{D}^{s}: D(\xi) \rightarrow D(T(M))$ denote the composite map

$$
D(\xi) \stackrel{\rho_{D}}{\rightarrow} D(T(M)) \stackrel{g^{s}}{\rightarrow} D(T(M)),
$$

where $\rho_{D}: D(\xi) \rightarrow D(T(M))$ is the bundle projection. Note that $\rho_{D}^{s}: D(\xi) \rightarrow$ $D(T(M))$ is a bundle projection.

In this step, we construct a homotopy $f_{t}:\left(h^{*} D(\xi), h^{*} S(\xi)\right) \rightarrow(D(\xi), S(\xi))$, $t \in[0,1]$, of the map $D(h)$ that satisfies the following properties.

8.2. Let $\varepsilon_{1}, \beta_{1}$ denote given positive numbers. Then for a given sufficiently large positive number $s$, we may assume that the following hold.

(a) Let $K_{1, s}^{\prime}$ denote the minimal subcomplex of $K_{1, s}$ that contains $D_{\varepsilon_{1}, \beta_{1}}(T(M))$. Then the map $f_{1}:\left(h^{*} D(\xi), h^{*} S(\xi)\right) \rightarrow(D(\xi), S(\xi))$ is split over $K_{1, s}^{\prime}$ with respect to the projection $\rho_{D}^{s}: D(\xi) \rightarrow D(T(M))$.

(b) The homotopy $f_{t}, t \in[0,1]$, has compact support.

(c) The homotopy $f_{t}, t \in[0,1]$, is $\left(\alpha_{1}, \delta_{1}\right)$-controlled over $\left(D(T(M)), \mathscr{F}_{D}\right)$. Here $\alpha_{1}=\eta^{-1} \Theta$, where $\eta$ comes from 8.1(a) and $\Theta$ comes from Lemma 7.1, and $\delta_{1}$ is a positive number satisfying $\lim _{s \rightarrow \infty} \delta_{1}=0$. (The control here is measured with respect to the bundle projection $\rho_{D}^{s}: D(\xi) \rightarrow D(T(M))$.) 
For a fixed but arbitrarily large positive number $s$, we let $L_{1}, L_{2}, L_{3}, \ldots$ denote the collection of all closed orbits of the geodesic flow $g^{t}: D(T(M)) \rightarrow$ $D(T(M)), t \in(-\infty, \infty)$, that satisfy the following properties.

8.3. (a) The length of each $L_{i}$ is less than $2 \eta^{-1} \Theta$.

(b) Each $L_{i}$, when projected onto $M$ under the bundle projection $D(T(M))$ $\rightarrow M$, intersects with $D_{\varepsilon_{1}, \beta_{1}}(T(M))^{\eta^{-1} \Theta, \delta}$ for $\delta$ fixed but very small.

Choose very small pairwise disjoint tubular neighborhoods $\left\{T_{i}\right\}$ for the $\left\{L_{i}\right\}$ in $D(T(M))$. Let $\dot{T}_{i}$ denote the topological boundary of $T_{i}$ in $D(T(M))$. Note that it follows from Addendum 3.5.5 and from 8.1(c) that there will be no loss of generality in assuming that the $T_{i}$ satisfy the following properties.

8.4. (a) Each $T_{i}$ is the underlying set of a subcomplex of $K_{1, s}$.

(b) The foliation $\mathscr{G}$ is uniformly $\left(\eta^{-1} \boldsymbol{\Theta}, 0\right)$-flat on the subset $\left(\left|K_{1, s}^{\prime}\right|-\right.$ $\left.\bigcup_{i} T_{i}\right) \cup\left(\bigcup_{i} \dot{T}_{i}\right)$, for $\delta>0$ sufficiently small.

It follows from Lemma 7.1 that $D(h)$ has the following control property.

8.5. $D(h): h^{*} D(\xi) \rightarrow D(\xi)$ is $\left(\Theta, \delta^{\prime}\right)$-controlled over $(D(T(M)), \mathscr{G})$, where $\delta^{\prime}$ is a positive number satisfying $\lim _{s \rightarrow \infty} \delta^{\prime}=0$. (The control data are measured here with respect to the bundle projection $\rho_{D}^{s}: D(\xi) \rightarrow D(T(M))$.)

Now it follows from 8.4 and 8.5 that we may apply Theorem 6.6 to get a homotopy $f_{t}:\left(h^{*} D(\xi), h^{*} S(\xi)\right) \rightarrow(D(\xi), S(\xi)), t \in\left[0, \frac{1}{2}\right]$, of $D(h)$ that satisfies the following properties.

8.6. (a) The homotopy $f_{t}, t \in\left[0, \frac{1}{2}\right]$, has compact support.

(b) The homotopy $f_{t}, t \in\left[0, \frac{1}{2}\right]$, is $\left(\eta^{-1} \Theta, \delta_{1}\right)$-controlled over $(D(T(M))$, $\mathscr{G}$ ), where $\delta_{1}$ is a positive number satisfying $\lim _{\delta^{\prime} \rightarrow 0} \delta_{1}=0$. (Here the control is measured with respect to the bundle projection $\rho_{D}^{s}: D(\xi) \rightarrow D(T(M))$.)

(c) Let $K_{1, s}^{\prime \prime}$ denote the minimal subcomplex of $K_{1, s}$ that contains the subset $\left(\left|K_{1, s}^{\prime}\right|-\bigcup_{i} T_{i}\right) \cup\left(\bigcup_{i} \dot{T}_{i}\right)$. Then $f_{1 / 2}:\left(h^{*} D(\xi), h^{*} S(\xi)\right) \rightarrow(D(\xi), S(\xi))$ is split over $K_{1, s}^{\prime \prime}$ with respect to the bundle projection $\rho_{D}^{s}: D(\xi) \rightarrow D(T(M))$.

For each $T_{i}$, set $\partial_{-} T_{i}=\partial T_{i} \cap S(T(M)), \partial_{+} T_{i}=\overline{\partial T_{i}-\partial_{-} T_{i}}, \wedge T_{i}=\partial_{+} T_{i} \cap$ $\partial_{-} T_{i}$, and let $X_{i}, \partial_{+} X_{i}, \partial_{-} X_{i}, \wedge X_{i}$ denote the subcomplexes of $K_{1, s}$ having the underlying sets $T_{i}, \partial_{+} T_{i}, \partial_{-} T_{i}, \wedge T_{i}$. It follows from 8.6(a) that there are only finitely many of the $X_{i}$ such that the map $f_{1 / 2}: h^{*} D(\xi) \rightarrow D(\xi)$ is split over $\partial_{+} X_{i}$ but not split over all of $X_{i}$. We denote this finite collection by $\left\{X_{i}: i \in I\right\}$. Since each $\partial_{-} T_{i}, T_{i}$ is the total space of a fiber bundle over $S^{1}$ having for fiber $B^{2 m-3}, B^{2 m-2}$, respectively (where $m=\operatorname{dim}(M)$ ), we may apply the splitting Theorem 6.2 to the map $f_{1 / 2}: h^{*} D(\xi) \rightarrow D(\xi)$, first over each of the $\left\{\partial_{-} X_{i}: i \in I\right\}$ and then over each of the $\left\{X_{i}: i \in I\right\}$, to get a homotopy $f_{t}: h^{*} D(\xi) \rightarrow D(\xi), t \in\left[\frac{1}{2}, 1\right]$, of $f_{1 / 2}$ that satisfies the following properties.

8.7. (a) $f_{t}, t \in\left[\frac{1}{2}, 1\right]$, is the constant homotopy over $D(T(M))-\bigcup_{i \in I} T_{i}$. 
(b) $f_{1}: h^{*} D(\xi) \rightarrow D(\xi)$ is split over all of $X_{i}$ for each $i \in I$.

Note that it follows from 8.6 that the homotopy $f_{t}: h^{*} D(\xi) \rightarrow D(\xi), t \in$ $[0,1]$, satisfies $8.2(a)-(c)$.

This completes Step I.

Step II. In this step, we construct a homotopy $g_{t}: h^{*} C(\xi) \rightarrow C(\xi), t \in[0,1]$, of $C(h)$ that satisfies the following properties.

8.8. Let $\varepsilon_{2}, \beta_{2}, \delta_{2}, \delta$ denote given positive numbers such that $\varepsilon_{1}^{-1} \ll \varepsilon_{2}^{-1}$ and $\beta_{1} \ll \beta_{2}$. Then if $\delta^{\prime} \in(0, \delta / 2)$ is choosen small enough, the homotopy $g_{t}, t \in[0,1]$, can be choosen to satisfy the following.

(a) The pair $\left(f_{t}, g_{t}\right), t \in[0,1]$, is an admissible homotopy pair. In fact, for each $x \in h^{*} S(\xi)$ and each $t \in[0,1]$, we have that $g_{t}$ maps ${ }_{\delta^{\prime}} \tau_{x}^{\prime \prime}$ homeomorphically onto ${ }_{\delta^{\prime}} \tau_{f_{t}(x)}^{\prime}$.

(b) For each $t \in[0,1], u \in\left[0,2 \delta^{\prime}\right]$, we have that $g_{t}\left({ }_{u} \dot{\tau}^{\prime \prime}\right) \subset{ }_{u} \dot{\tau}^{\prime}$. Moreover, the homotopy $g_{t}:\left({ }_{2 \delta^{\prime}} \tau^{\prime \prime},{ }_{2 \delta^{\prime}} \dot{\tau}^{\prime \prime}\right) \rightarrow\left({ }_{2 \delta^{\prime}} \tau^{\prime},{ }_{2 \delta^{\prime}} \dot{\tau}^{\prime}\right), t \in[0,1]$, is $\left(5 \alpha_{1}, 5 \delta_{1}\right)$ controlled over $\left(S(T(M)), \mathscr{G}_{S}\right)$, where $\mathscr{G}_{S}$ denotes the restriction of $\mathscr{G}$ to $S(T(M))$. (Here the control is measured with respect to the bundle projection that is the composite map

$$
\left.{ }_{2 \delta^{\prime}} \tau^{\prime} \stackrel{\rho_{\tau^{\prime}}}{\rightarrow} S(\xi) \stackrel{\rho_{D}^{s}}{\longrightarrow} S(T(M)) .\right)
$$

(c) Let $K_{2}^{\prime}$ denote the minimal subcomplex of $K_{2}$ that contains ${ }_{\delta, 2 \delta} \tau \cup$ $\left[C_{\varepsilon_{2}, \beta_{2}}(T(M)){ }_{\delta} \tau\right]$. Then the map $g_{1}: h^{*} C(\xi)-h^{*} S(\xi) \rightarrow C(\xi)-S(\xi)$ is split over the subcomplex $K_{2}^{\prime}$ with respect to the projection $\rho_{C}: C(\xi)-S(\xi) \rightarrow$ $C(T(M))-S(T(M))$.

(d) The homotopy $g_{t}: h^{*} C(\xi)-h^{*} S(\xi) \rightarrow C(\xi)-S(\xi), t \in[0,1]$, is $\left(\alpha_{2}, \delta_{2}\right)-$ controlled over $\left(C(T(M))-{ }_{2 \delta^{\prime}} \tau, \mathscr{F}_{C}\right)$. Here $\alpha_{2}$ is a positive number that is independent of $\varepsilon_{2}, \beta_{2}, \delta_{2}$. (Here control is measured with respect to the bundle projection $\rho_{C}: C(\xi)-S(\xi) \rightarrow C(T M)-S(T M)$.)

We shall construct separately the two pieces $\left.g_{t}\right|_{2 \delta^{\prime}} \tau^{\prime \prime}, t \in[0,1]$, and $g_{t} \mid h^{*} C(\xi)-{ }_{2 \delta^{\prime}} \tau^{\prime \prime}, t \in[0,1]$, of the homotopy $g_{t}, t \in[0,1]$.

We shall first construct the piece $\left.g_{t}\right|_{2 \delta^{\prime}} \tau^{\prime \prime}, t \in[0,1]$. Define maps $s^{\prime}:{ }_{2 \delta^{\prime}} \tau^{\prime}-$ $S(\xi) \rightarrow\left(0,2 \delta^{\prime}\right]$ and $s^{\prime \prime}:{ }_{2 \delta^{\prime}} \tau^{\prime \prime}-h^{*} S(\xi) \rightarrow\left(0,2 \delta^{\prime}\right]$ as follows: $s^{\prime}\left(x^{\prime}\right)=t$, $s^{\prime \prime}\left(x^{\prime \prime}\right)=t$ for all $x^{\prime} \in{ }_{t} \dot{\tau}, x^{\prime \prime} \in i^{\prime \prime}{ }^{\prime \prime}$ with $t \in\left(0,2 \delta^{\prime}\right]$. Set

$$
\rho^{\prime}=\left[\left.\rho_{\tau^{\prime}}\right|_{2 \delta^{\prime}} \tau^{\prime}-S(\xi)\right] \times s^{\prime} \text { and } \rho^{\prime \prime}=\left[\left.\rho_{\tau^{\prime \prime}}\right|_{2 \delta^{\prime}} \tau^{\prime \prime}-h^{*} S(\xi)\right] \times s^{\prime \prime},
$$

where $\rho_{\tau^{\prime}}: \tau^{\prime} \rightarrow S(\xi)$ and $\rho_{\tau^{\prime \prime}}: \tau^{\prime \prime} \rightarrow h^{*} S(\xi)$ are the bundle projections. Note that both of

$$
\rho^{\prime}:{ }_{2 \delta^{\prime}} \tau^{\prime}-S(\xi) \rightarrow S(\xi) \times\left(0,2 \delta^{\prime}\right]
$$

and

$$
\rho^{\prime \prime}:{ }_{2 \delta^{\prime}} \tau^{\prime \prime}-h^{*} S(\xi) \rightarrow h^{*} S(\xi) \times\left(0,2 \delta^{\prime}\right]
$$

are fiber bundle projections and the map $C(h):{ }_{2 \delta^{\prime}} \tau^{\prime \prime}-h^{*} S(\xi) \rightarrow{ }_{2 \delta^{\prime}} \tau^{\prime}-S(\xi)$ maps each fiber of $\rho^{\prime \prime}$ homeomorphically onto a fiber of $\rho^{\prime}$. Moreover, the map 
$C(h):{ }_{2 \delta^{\prime}} \tau^{\prime \prime}-h^{*} S(\xi) \rightarrow{ }_{2 \delta^{\prime}} \tau^{\prime}-S(\xi)$ covers the map $S(h) \times 1: h^{*} S(\xi) \times\left(0,2 \delta^{\prime}\right] \rightarrow$ $S(\xi) \times\left(0,2 \delta^{\prime}\right]$. It follows that the homotopy $f_{t}, t \in[0,1]$, of 8.2 can be lifted to a covering homotopy $\hat{f}_{t}:{ }_{2 \delta} \tau^{\prime \prime}-h^{*} S(\xi) \rightarrow{ }_{2 \delta^{\prime}} \tau^{\prime}-S(\xi), t \in[0,1]$, that satisfies the following properties.

(a) $\hat{f}_{0}=\left.C(h)\right|_{2 \delta^{\prime}} \tau^{\prime \prime}-h^{*} S(\xi)$.

(b) For each $t \in[0,1]$ and each $x \in h^{*} S(\xi), u \in\left(0,2 \delta^{\prime}\right]$, we have that $\hat{f}_{t}$ maps the fiber $\left(\rho^{\prime \prime}\right)^{-1}(x, u)$ homeomorphically onto the fiber $\left(\rho^{\prime}\right)^{-1}\left(f_{t}(x), u\right)$.

We can now define the piece $\left.g_{t}\right|_{2 \delta^{\prime}} \tau^{\prime \prime}, t \in[0,1]$, by the formulae of 8.10.

8.10. (a) $g_{t} \mid h^{*} S(\xi)=f_{t}$ for all $t \in[0,1]$.

(b) $\left.g_{t}\right|_{\delta^{\prime}} \tau^{\prime \prime}-h^{*} S(\xi)=\left.\hat{f}_{t}\right|_{\delta^{\prime}} \tau^{\prime \prime}-h^{*} S(\xi)$ for all $t \in[0,1]$.

(c) For each $v \in\left[\delta^{\prime}, 2 \delta^{\prime}\right]$, each $x \in{ }_{v} \dot{\tau}^{\prime \prime}$, and each $t \in[0,1]$, set $g_{t}(x)=$ $\hat{f}_{t^{\prime}}(x)$, where $t^{\prime}=t\left(2 \delta^{\prime}-v\right) / \delta^{\prime}$.

Note that it follows from $8.9,8.10,8.2$, that $\left.g_{t}\right|_{2 \delta^{\prime}} \tau^{\prime \prime}, t \in[0,1]$, satisfies the following properties if $\delta^{\prime}$ is choosen sufficiently small.

8.11. (a) $\left.g_{t}\right|_{2 \delta^{\prime}} \tau^{\prime \prime}, t \in[0,1]$, satisfies $8.8(\mathrm{a})$ and (b).

(b) $\left.g_{t}\right|_{2 \delta^{\prime}} \dot{t}^{\prime \prime}, t \in[0,1]$, is the constant homotopy.

In order to construct the piece $g_{t} \mid h^{*} C(\xi)-{ }_{2 \delta^{\prime}} \tau^{\prime \prime}, t \in[0,1]$, of the homotopy $g_{t}, t \in[0,1]$, we must apply the splitting theorems of $\S 6$ to three different projections and foliations $p_{i}: E_{i} \rightarrow M_{i}, \mathscr{F}_{i}, i=1,2,3$. We begin by describing these projections and foliations.

8.12. Description of $p_{1}: E_{1} \rightarrow M_{1}$ and $\mathscr{F}_{1}$. Let $\mu$ denote a positive number and let $y<z$ denote positive integers. Below we will place restrictions on $\mu, y, z$, but for the present, we let $\mu, y, z$ be arbitrary. Define an open subset $U_{1} \subset \gamma$ as follows.

(a) $U_{1}=\bigcup_{i=y}^{z}\left(\mu_{i}, \mu_{i}^{\prime} \gamma-\partial_{\mu_{i}, \mu_{i}^{\prime}} \gamma\right)$, where $\mu_{i}=2 i \mu, \mu_{i}^{\prime}=\mu_{i}+\mu$.

For each $t>0$, we define a diffeomorphism $w_{t}:{ }_{t} \dot{\gamma} \times(0, \infty) \rightarrow \gamma-\bar{S}(T(M))$ as follows. For each $(x, t) \in_{t} \dot{\gamma} \times(0, \infty)$, set $w_{t}(x, t)=x$. For each $x \in \in_{t} \dot{\gamma}$, the curve $f(s)=w_{t}(x, s)$ is a unit speed geodesic in the fiber $\gamma_{\rho_{\gamma}(x)}$ of $\gamma$ that is perpendicular to ${ }_{t} \dot{\gamma}_{\rho_{\gamma}(x)}$ (where $\rho_{\gamma}: \gamma \rightarrow \bar{S}(T(M)$ ) is the bundle projection). Let each ${ }_{\mu_{i}} \dot{\gamma} x\left(\mu_{i}, \mu_{i}^{\prime}\right)$ be equipped with the product metric, where ${ }_{\mu_{i}} \dot{\gamma}$ inherits its metric from $\gamma$. Then push these product metrics forward into $U_{1}$ by the maps $\left.w_{\mu_{i}}\right|_{\mu_{i}} \dot{\gamma} \times\left(\mu_{i}, \mu_{i}^{\prime}\right)$ to get a metric on $U_{1}$, and extend this metric (in any way) to the Riemann metric $\langle,\rangle_{1}$ on all of $\gamma$. Define $M_{1}$ and $p_{1}: E_{1} \rightarrow M_{1}$ as follows.

(b) $M_{1}$ is the manifold $\gamma$ equipped with the metric $\langle,\rangle_{1}$.

(c) $p_{1}: E_{1} \rightarrow M_{1}$ is equal to the bundle projection $\rho: C(\xi)-S(\xi) \rightarrow \gamma$. Define a foliation $\mathscr{F}_{1}$ of the open set $U_{1}$ as follows.

(d) The leaves of $\mathscr{F}_{1}$ have the form $L \cap\left({ }_{\mu_{i}, \mu_{i}^{\prime}} \gamma-\partial_{\mu_{i}, \mu_{i}^{\prime}} \gamma\right)$ for some $i=$ $y, y+1, \ldots, z$, where $L$ is a leaf of the asymptotic foliation $\mathscr{F}_{C}$. 
8.13. Description of $p_{2}: E_{2} \rightarrow M_{2}$ and $\mathscr{F}_{2}$. Define a closed subset $U_{2} \subset \gamma$ as follows.

(a) $U_{2}=\bigcup_{i=y \quad v_{i}, v_{i}^{\prime}}^{z-1} \gamma$, where $v_{i}=\mu_{i}^{\prime}-\frac{1}{4} \mu, v_{i}^{\prime}=\mu_{i+1}+\frac{1}{4} \mu$.

Define $p_{2}: E_{2} \rightarrow M_{2}$ as follows.

(b) $M_{2}=\bigcup_{i=y}^{z-1} \dot{\gamma}$, where the Riemann metric on $M_{2}$ is inherited from $\gamma$.

(c) Set $E_{2}=\rho^{-1}\left(U_{2}\right)$. Set $p_{2}$ equal to the composite map

$$
E_{2} \stackrel{\rho}{\rightarrow} U_{2} \stackrel{g}{\rightarrow} \bigcup_{i=y}^{z-1} v_{i} \dot{\gamma} x\left[v_{i}, v_{i}^{\prime}\right] \stackrel{\text { proj }}{\rightarrow} \bigcup_{i=y}^{z-1} v_{i} \dot{\gamma},
$$

where for each $i=y, y+1, \ldots, z-1$, we have that $g \mid w_{v_{i}}\left({ }_{v_{i}} \dot{\gamma} \times\left[v_{i}, v_{i}^{\prime}\right]\right)=$ $w_{v_{i}}^{-1} \mid w_{v_{i}}\left(v_{i} \dot{\gamma} \times\left[v_{i}, v_{i}^{\prime}\right]\right)$. (Recall that $\rho: C(\xi)-S(\xi) \rightarrow \gamma$ is the bundle projection.)

Define a foliation $\mathscr{F}_{2}$ of the set $M_{2}$ as follows.

(d) The leaves of $\mathscr{F}_{2}$ have the form $L \cap{ }_{v_{i}} \dot{\gamma}$, where $L$ is a leaf of the asymptotic foliation $\mathscr{F}_{C}$ and $i=y, y+1, \ldots, z-1$.

8.14. Description of $p_{3}: E_{3} \rightarrow M_{3}, \mathscr{F}_{3}$. (a) $M_{3}=\bar{S}(T(M))$.

(b) $\mathscr{F}_{3}=\overline{\mathscr{G}}$. Here $\overline{\mathscr{G}}$ is the 1-dimensional foliation of $\bar{S}(T(M))$ by the marking geodesics (see (3.4.4)).

(c) $E_{3}=\rho^{-1}\left({ }_{x} \gamma\right)$, where $x=2 y \mu+10^{-1} \mu$ and $y, \mu$ come from 8.12. (Here $\rho: C(\xi)-S(\xi) \rightarrow \gamma$ is the bundle projection.)

(d) $p_{3}$ is equal to the composite map

$$
E_{3} \stackrel{\rho}{\rightarrow}{ }_{x} \gamma \stackrel{\rho_{y}}{\rightarrow} S(T(M)) .
$$

In the remainder of this step, we will assume that the triangulations $K_{2}, K_{3}$ satisfy the following properties in addition to $8.1(\mathrm{~b})$ and (c).

8.15. (a) For each simplex $\Delta \in K_{3}$, we have that $\rho_{\gamma}^{-1}(\Delta) \cap_{x} \dot{\gamma}$ is the underlying set of a subcomplex of $K_{2}$. Here $x$ is the number given in 8.14(c).

(b) $U_{2}$ is the underlying set of a subcomplex of $K_{2}$. Here $U_{2}$ comes from 8.13.

(c) There is a triangulation $T_{2}$ for $M_{2}$ such that, for each simplex $\Delta \in T_{2}$, we have that $q_{2}^{-1}(\Delta)$ is the underlying subset of a subcomplex of $K_{2}$. (Here $q_{2}: U_{2} \rightarrow M_{2}$ is defined to be the composite map

$$
v_{i}, v_{i}^{\prime} \stackrel{w_{v_{i}}^{-1}}{\rightarrow} v_{i} \dot{\gamma} \times\left[v_{i}, v_{1}^{\prime}\right] \stackrel{\text { proj }}{\longrightarrow} v_{i} \dot{\gamma}
$$

on the subset ${ }_{v_{i}, v_{i}^{\prime}} \gamma \subset U_{2}$.)

(d) $d\left(T_{2}\right)$ may be assumed to be arbitrarily small.

We have now enough new notation to begin the construction of the piece $g_{t} \mid h^{*} C(\xi)-{ }_{2 \delta^{\prime}} \tau^{\prime \prime}, t \in[0,1]$, of the homotopy $g_{t}, t \in[0,1]$. The first part of this construction consists of two applications of Theorem 6.6, one such application for each of the projections $p_{i}: E_{i} \rightarrow M_{i}, i=1,2$. We will need the 
following uniformity properties (which follow from Addendum 3.15.1) for the foliations $\mathscr{F}_{i}, i=1,2$, in order to carry out these applications of Theorem 6.6 .

8.16. Uniform flatness for $\mathscr{F}_{i}, i=1,2$. Given any number $\alpha>0$ and any compact set $X \subset M$, there is a number $\Gamma>0$ such that the following properties hold.

(a) $\Gamma$ depends only on $\alpha, X, \mu$, where $\mu$ comes from 8.12.

(b) Suppose in 8.12 we have that $y \geq \Gamma$. Then we have that $\mathscr{F}_{1}$ is uniformly $(\alpha, \sigma)$-flat on the subset $U_{1}^{-\left(10^{-4} \mu\right)} \cap X^{\prime}$ of $M_{1}$, and $\mathscr{F}_{2}$ is uniformly $(\alpha, \sigma)$ flat on the subset $M_{2} \cap X^{\prime}$ of $M_{2}$. Here $\sigma$ is the number in 8.1(a), $X^{\prime} \subset$ $C(T(M))$ is the preimage of $X$ under the bundle projection $C(T(M)) \rightarrow M$, and $U_{1}^{-\left(10^{-4} \mu\right)}$ is the set of all $x \in U_{1}$ a distance greater than $10^{-4} \mu$ from $\gamma-U_{1}$.

We shall first apply Theorem 6.6 to the map $C(h): h^{*} C(\xi)-h^{*} S(\xi) \rightarrow C(\xi)-$ $S(\xi)$ when the foliated control data for this map is computed with respect to $p_{1}: E_{1} \rightarrow M_{1}$ and $\mathscr{F}_{1}$. Note that it follows from Lemma 7.1, 8.12, and Lemma 3.16 that if $\mu$ of 8.12 is chosen to satisfy $8.17(\mathrm{a})$, then 8.17 (b) will also hold.

8.17. (a) $\mu>10^{4} \Theta$.

(b) The map $C(h): h^{*} C(\xi)-h^{*} S(\xi) \rightarrow E_{1}$ is $(\Theta, 0)$-controlled over $\left(U_{1}^{-\left(10^{-4} \mu\right)}, \mathscr{F}_{1}\right)$. (Here the control is the control data and is computed with respect to the bundle projection $p_{1}$.)

Note that if in 8.16 we choose $\alpha>\eta^{-1} \Theta$, if in 8.12 we choose $\mu>10^{4} \Theta$ and choose $y \geq \Gamma$, and if in 8.16 we choose the compact subset $X \subset M$ so large that its preimage $X^{\prime}$ is much larger than the support for the map $C(h)$, then 8.16 and 8.17 together show that the hypotheses for applying Theorem 6.6 to $C(h)$ over $\left(U_{1}^{-\left(10^{-4} \mu\right)}, \mathscr{F}\right)$ are satisfied. Thus by Theorem 6.6 there is a homotopy $r_{t}: h^{*} C(\xi)-h^{*} S(\xi) \rightarrow E_{1}, t \in[0,1]$, of $C(h) \mid\left(h^{*} C(\xi)-h^{*} S(\xi)\right)$ that satisfies the following properties.

8.18. (a) Let $K_{2,1}$ denote the minimal subcomplex of $K_{2}$ that contains the set $U_{1}^{-\left(10^{-3} \mu\right)}$. Then $r_{1}: h^{*} C(\xi)-h^{*} S(\xi) \rightarrow E_{1}$ is split over $K_{2,1}$ with respect to the bundle projection $p_{1}$.

(b) The homotopy $r_{t}, t \in[0,1]$, has compact support. The support of $r_{t}$, $t \in[0,1]$, in fact lies over a subset of $X^{\prime} \cap U_{1}^{-\left(10^{-4} \mu\right)}$ that is much smaller than $X^{\prime}$.

(c) Let $u_{1}$ be a fixed but arbitrarily small positive number. We may assume that the homotopy $r_{t}, t \in[0,1]$, is $\left(\eta^{-1} \Theta, u_{1}\right)$-controlled over $\left(U_{1}^{-\left(10^{-4} \mu\right)}, \mathscr{F}_{1}\right)$, with respect to the projection $p_{1}: E_{1} \rightarrow M_{1}$.

This completes the application of Theorem 6.6 for the case when foliated control data is measured with respect to $p_{1}: E_{1} \rightarrow M_{1}$ and $\mathscr{F}_{1}$. Now we wish to apply Theorem 6.6 to the map $r_{1}: h^{*} C(\xi)-h^{*} S(\xi) \rightarrow C(\xi)-S(\xi)$ when 
the foliated control data is measured with respect to $p_{2}: E_{2} \rightarrow M_{2}$ and $\mathscr{F}_{2}$. Note that if we define a map $F: N_{2} \rightarrow E_{2}$ by 8.19 (a), then it will follow from Lemma 7.1, 8.17(b), 8.18(b) and (c), 8.15, and Lemma 3.16 that $f$ satisfies 8.19 (b)-(d).

(a) $N_{2}=\left(r_{1}\right)^{-1}\left(E_{2}\right) ; F=r_{1} \mid N_{2}$.

(b) $F:\left(N_{2}, \partial N_{2}\right) \rightarrow\left(E_{2}, \partial E_{2}\right)$ is a homotopy equivalence of manifold pairs that has compact support. The support of $F$ in fact lies over a subset of $X^{\prime} \cap M_{2}$ that is much smaller than $X^{\prime}$.

(c) $F: \partial N_{2} \rightarrow \partial E_{2}$ is split over the entire triangulation $T_{2}$ with respect to the bundle projection $E_{2} \stackrel{p_{2}}{\rightarrow} M_{2}$.

(d) $F: N_{2} \rightarrow E_{2}$ is $\left(e^{4 \mu} \eta^{-1} \Theta, u_{1}^{\prime}\right)$-controlled over $\left(M_{2}, \mathscr{F}_{2}\right)$, where $u_{1}^{\prime}$ is a positive number satisfying $\lim _{u_{1} \rightarrow 0} u_{1}^{\prime}=0$. (Here the data are measured with respect to the bundle projection $p_{2}: E_{2} \rightarrow M_{2}$.)

Note that if in 8.16 we choose $\alpha>e^{4 \mu} \eta^{-2} \Theta$, and if in 8.12 and 8.13 we choose $y \geq \Gamma$, where $\Gamma$ comes from 8.16, then 8.16 and 8.19 together show that the hypotheses for applying Theorem 6.6 to $F: N_{2} \rightarrow E_{2}$ over $\left(M_{2}, \mathscr{F}_{2}\right)$ are satisfied. Thus, by Theorem 6.6 there is a homotopy $F_{t}:\left(N_{2}, \partial N_{2}\right) \rightarrow$ $\left(E_{2}, \partial E_{2}\right), t \in[0,1]$, of $F$ that satisfies the following properties.

8.20. (a) $F_{t}: \partial N_{2} \rightarrow \partial E_{2}, t \in[0,1]$, is the constant homotopy.

(b) The homotopy $F_{t}, t \in[0,1]$, has compact support. The support of the homotopy $F_{t}, t \in[0,1]$, does in fact lie over a subset of $X^{\prime} \cap M$, that is much smaller than $X^{\prime}$.

(c) The homotopy $F_{t}, t \in[0,1]$, is $\left(e^{4 \mu} \eta^{-2} \Theta, u_{1}^{\prime \prime}\right)$-controlled over $\left(M_{2}, \mathscr{F}_{2}\right)$, where $u_{1}^{\prime \prime}$ is a positive number such that limit $u_{1}^{\prime \prime}=0$ as $u_{1} \rightarrow 0$ and as $d\left(T_{2}\right) \rightarrow 0($ see $8.15(\mathrm{~d}))$.

(d) $F_{1}:\left(N_{2}, \partial N_{2}\right) \rightarrow\left(E_{2}, \partial E_{2}\right)$ is split over the entire triangulation $T_{2}$, with respect to the projection $p_{2}: E_{2} \rightarrow M_{2}$.

Consider the cell structure $A$ on $U_{2}$ consisting of all

$$
\left\{\left(\left.q_{2}\right|_{v_{i}, v_{i}^{\prime}} \gamma\right)^{-1}(\Delta), \partial_{+}\left(\left.q_{2}\right|_{v_{i}, v_{i}^{\prime}} \gamma\right)^{-1}(\Delta), \partial_{-}\left(\left.q_{2}\right|_{v_{i}, v_{i}^{\prime}} \gamma\right)^{-1}(\Delta): \Delta \in T_{2}\right\}
$$

where $\partial_{ \pm}\left(\left.q_{2}\right|_{v_{i}, v_{i}^{\prime}} \gamma\right)^{-1}(\Delta)$ denote the two connected components of $\left(\left.q_{2}\right|_{v_{i}, v_{i}} \gamma\right)^{-1}(\Delta) \cap \partial U_{2}$ and where $q_{2}$ comes from 8.15. By 8.20(d), we have that $F_{1}$ is split over $A$. By 8.15 , we have that $A$ is subdivided by a subcomplex $A^{\prime}$ of $K_{2}$. By $8.20(a), 8.18$ (a) $8.12,8.13$, we have that $F_{1}$ is already split over $\partial A^{\prime}$ (with respect to $\rho \mid E_{2}: E_{2} \rightarrow U_{2}$ ), where $\partial A^{\prime}$ denotes the subcomplex of $A^{\prime}$ that is contained in $\partial U_{2}$. Thus, by using Wall's $\pi-\pi$ splitting theorem, we may choose a further homotopy $F_{t}: N_{2} \rightarrow E_{2}, t \in[1,2]$, of $F_{1}$ that is constant on $\partial N_{2}$ such that each $F_{t}, t \in[1,2]$, is split over $T_{2}$ (with respect to $p_{2}: E_{2} \rightarrow M_{2}$ ) and such that $F_{2}$ is split over $A^{\prime}$ (with respect to $\left.\rho \mid E_{2}: E_{2} \rightarrow U_{2}\right)$. Set $\bar{F}_{t}=F_{2 t}$ for $t \in[0,1]$. Then we can summarize the results of this paragraph by 8.21 . 
8.21. (a) $\bar{F}_{t}: N_{2} \rightarrow E_{2}, t \in[0,1]$, satisfies $8.20\left(\right.$ a) and (b), when $\bar{F}_{t}$ replaces $F_{t}$ in 8.20 .

(b) Let $K_{2,2}$ denote the subcomplex of $K_{2}$ with underlying set equal to $U_{2}$. Then $\bar{F}_{1}$ is split over $K_{2,2}$ (with respect to $\rho \mid E_{2}: E_{2} \rightarrow U_{2}$ ).

(c) The homotopy $\bar{F}_{t}, t \in[0,1]$, is $\left(e^{4 \mu} \eta^{-2} \Theta+d\left(T_{2}\right), u_{1}^{\prime \prime}+d\left(T_{2}\right)\right)$-controlled over $\left(M_{2}, \mathscr{F}_{2}\right)$. (Here control is measured with respect to the bundle projection $p_{2}$.)

This completes our second application of Theorem 6.6, for the special case when the control data is measured with respect to the projection $p_{2}$ and the foliation $\mathscr{F}_{2}$. We come now to the third (and last of this step) application of Theorem 6.6, for the special case when the control data are measured with respect to the projection $p_{3}: E_{3} \rightarrow M_{3}$ and foliation $\mathscr{F}_{3}$ of 8.14.

Note that it follows from 8.12-8.15, 8.18, and the fact that $\rho_{\gamma}: \gamma \rightarrow \bar{S}(T(M))$ is nonexpanding in the directions of the leaves of $\mathscr{F}_{C}$, that if $G: N_{3} \rightarrow E_{3}$ is defined by $8.22(\mathrm{a})$, then $G$ must satisfy $8.22(\mathrm{~b})-(\mathrm{d})$.

8.22. (a) $N_{3}=\left(r_{1}\right)^{-1}\left(E_{3}\right) ; G=r_{1} \mid N_{3}$.

(b) $G:\left(N_{3}, \partial N_{3}\right) \rightarrow\left(E_{3}, \partial E_{3}\right)$ is a homotopy equivalence of manifold pairs that has compact support.

(c) $G: N_{3} \rightarrow E_{3}$ is $\left(e^{4 \mu} \eta^{-1} \Theta, u_{1}^{\prime}\right)$-controlled over $\left(M_{3}, \mathscr{F}_{3}\right)$, where $u_{1}^{\prime}$ is a positive number satisfying $\lim _{u_{1} \rightarrow 0} u^{\prime}=0$ and $u_{1}$ comes from $8.18(\mathrm{c})$. (Here the control data are measured with respect to the projection $p_{3}$.)

(d) $G: \partial N_{3} \rightarrow \partial E_{3}$ is split over the entire triangulation $K_{3}$ with respect to the bundle projection $p_{3}: \partial E_{3} \rightarrow M_{3}$.

Let $\alpha, \varepsilon_{2}^{\prime}, \beta_{2}^{\prime}$ denote given positive numbers. Let $\left\{L_{i}: i=1,2, \ldots\right\}$ denote all the closed leaves of $\mathscr{F}_{3}$ that have length less than $2 \alpha$ and that intersect with $\bar{S}_{\varepsilon_{2}^{\prime}, \beta_{2}^{\prime}}(T(M))^{\alpha, \delta}$ for $\delta>0$ fixed but very small, and let $\left\{T_{i}: i=\right.$ $1,2, \ldots\}$ denote arbitrarily small pairwise disjoint tubular neighborhoods for the $\left\{L_{i}: i=1,2, \ldots\right\}$ in $M_{3}$. Note that it follows from (3.4.4) and 8.1(b) that there will be no loss of generality in assuming that the following properties hold.

8.23. (a) Each $T_{i}$ is the underlying set of a subcomplex of $K_{3}$.

(b) The foliation $\mathscr{F}_{3}$ is uniformly $(\alpha, 0)$-flat on the subset $X$, where

$$
X=\left[\bar{S}_{\varepsilon_{2}^{\prime}, \beta_{2}^{\prime}}(T(M))-\bigcup_{i=1}^{\infty} T_{i}\right] \cup\left[\bigcup_{i=1}^{\infty} \partial T_{i}\right] .
$$

Note that if $\alpha$ of 8.23 is choosen to satisfy $\alpha>e^{4 \mu} \eta^{-2} \Theta$ and if $u_{1}$ of 8.18 is choosen sufficiently small (see $8.22(\mathrm{c})$ ), then 8.22 and 8.23 together show that we may apply Theorem 6.6 to get a homotopy $G_{t}:\left(N_{3}, \partial N_{3}\right) \rightarrow\left(E_{3}, \partial E_{3}\right)$, $t \in[0,1]$, of $G$ that satisfies the following properties.

8.24. (a) The homotopy $G_{t}, t \in[0,1]$, has compact support. Moreover, the restricted homotopy $G_{t}: \partial N_{3} \rightarrow \partial E_{3}, t \in[0,1]$, is the constant homotopy. 
(b) Let $K_{3}^{\prime}$ denote the minimal subcomplex of $K_{3}$ that contains the subset $X$ of $8.23(\mathrm{~b})$. Then $G_{1}:\left(N_{3}, \partial N_{3}\right) \rightarrow\left(E_{3}, \partial E_{3}\right)$ is split over $K_{3}^{\prime}$ with respect to the projection $p_{3}$.

(c) The homotopy $G_{t}, t \in[0,1]$, is $\left(e^{4 \mu} \eta^{-2} \Theta, u_{1}^{\prime \prime}\right)$-controlled of $\left(M_{3}, \mathscr{F}_{3}\right)$, where $u_{1}^{\prime \prime}$ is a positive number satisfying $\lim _{u_{1}^{\prime} \rightarrow 0} u_{1}^{\prime \prime}=0$ and $u_{1}^{\prime}$ comes from 8.22. (Here the control data is measured with respect to the projection $p_{3}$.)

Let $\left\{X_{i}: i=1,2, \ldots\right\}$ denote the subcomplexes of $K_{3}$ that have the tubular neighborhoods $\left\{T_{i}: i=1,2, \ldots\right\}$ for underlying sets (see 8.23(a)). We now apply Theorem 6.2 to $G_{1}$ over each subcomplex $X_{i}$ of $K_{3}$ to get a homotopy $G_{t}:\left(N_{3}, \partial N_{3}\right) \rightarrow\left(E_{3}, \partial E_{3}\right), t \in[1,2]$, of $G_{1}$ that satisfies the following properties.

8.25. (a) Let $Y$ denote the preimage of $M_{3}-\bigcup_{i=1}^{\infty} T_{i}$ under the map $p_{3} \circ$ $G_{1}: N_{3} \rightarrow M_{3}$. Then the restricted homotopy $G_{t} \mid Y \cup \partial N_{3}, t \in[1,2]$, is the constant homotopy. Moreover, $G_{t}, t \in[1,2]$, has compact support.

(b) $G_{2}$ is split over each of the subcomplexes $\left\{X_{i}: i=1,2, \ldots\right\}$ with respect to the projection $p_{3}$.

Now $8.24,8.25,8.15(\mathrm{a})$, and $8.18(\mathrm{a})$ make it possible for us to apply Wall's $\pi-\pi$ splitting theorem (see [64]) inductively over the simplices of $K_{2}$ that are contained in ${ }_{x} \gamma$ of $8.15(\mathrm{a})$ to get a homotopy $G_{t}:\left(N_{3}, \partial N_{3}\right) \rightarrow\left(E_{3}, \partial E_{3}\right)$, $t \in[2,3]$, of $G_{2}$ satisfying: $G_{t} \mid \partial N_{3}, t \in[2,3]$, is the constant homotopy; $G_{t}, t \in[2,3]$, has compact support; for each $t \in[2,3]$, the map $G_{t}$ is split over the subcomplex $K_{3}^{\prime} \cup\left(\bigcup_{i=1}^{\infty} X_{i}\right)$ with respect to the projection $p_{3}$; the map $G_{3}$ is split over the minimal subcomplex of $K_{2}$ that contains the subset $\rho_{\gamma}^{-1}\left(\left|K_{3}^{\prime}\right| \cup\left(\bigcup_{i=1} T_{i}\right)\right)$ (this splitting is with respect to the projection $\left(p_{2} \mid E_{3}\right): E_{3}$ $\left.\rightarrow{ }_{x} \gamma\right)$. Set $\bar{G}_{t}=G_{3 t}$ for $t \in[0,1]$. Then we can summarize the results of this paragraph and of 8.24 and 8.25 as follows.

8.26. (a) $\bar{G}_{0}=G$. The homotopy $\bar{G}_{t}, t \in[0,1]$, has compact support. The restricted homotopy $\bar{G}_{t} \mid \partial N_{3}, t \in[0,1]$, is the constant homotopy.

(b) $\bar{G}_{1}: N_{3} \rightarrow E_{3}$ is split over the minimal subcomplex of $K_{2}$ that contains the set $\rho_{\gamma}^{-1}\left(\bar{S}_{\varepsilon_{2}^{\prime}, \beta_{2}^{\prime}}(T(M))\right) \cap_{x} \gamma$. (The splitting here is with respect to the projection $\left(p_{2} \mid E_{3}\right): E_{3} \rightarrow{ }_{x} \gamma$.)

(c) The homotopy $\bar{G}_{t}, t \in[0,1]$, is $\left(e^{4 \mu} \eta^{-2} \Theta+d\left(K_{3}\right), u_{1}^{\prime \prime}+d\left(K_{3}\right)\right)$-controlled over $\left(M_{3}, \mathscr{F}_{3}\right)$. (Here the control data are measured with respect to the projection $p_{3}$.)

We can now complete Step II by defining the piece $g_{t} \mid\left(h^{*} C(\xi)-{ }_{2 \delta^{\prime}} \tau^{\prime \prime}\right)$, $t \in[0,1]$ - of the homotopy $g_{t}, t \in[0,1]$, desired in 8.8-as follows. (See $8.19,8.22$ for $N_{2}, N_{3}$.) 
8.27 .

$$
g_{t}(x)= \begin{cases}r_{2 t}(x), & \text { if } t \in\left[0, \frac{1}{2}\right], \\ r_{1}(x), & \text { if } t \in\left[\frac{1}{2}, 1\right] \text { and } x \notin N_{2} \cup N_{3}, \\ \bar{F}_{t^{\prime}}(x), & \text { with } t^{\prime}=2\left(t-\frac{1}{2}\right), \text { if } t \in\left[\frac{1}{2}, 1\right] \text { and } x \in N_{2}, \\ \bar{G}_{t^{\prime}}(x), & \text { with } t^{\prime}=2\left(t-\frac{1}{2}\right), \text { if } t \in\left[\frac{1}{2}, 1\right] \text { and } x \in N_{3} .\end{cases}
$$

We assume that the following relations exist between the $\delta^{\prime}, \delta, \varepsilon_{2}, \beta_{2}$ of 8.8 , the $\mu, z, y, x$ of $8.12,8.13,8.14$, and the $\varepsilon_{2}^{\prime}, \beta_{2}^{\prime}$ of 8.23 . (Note that the following relations are made possible by Lemma 3.14.)

8.28. (a) Given $y, \mu$, we choose $\delta$ sufficiently small so that ${ }_{3 y \mu} \gamma \cap_{2 \delta} \tau=\varnothing$.

(b) Given $\varepsilon_{2}, \beta_{2}$, we choose $\varepsilon_{2}^{\prime}$ sufficiently small and choose $\beta_{2}^{\prime}$ sufficiently large so that

$$
\rho_{\gamma}^{-1}\left(\bar{S}_{\varepsilon_{2}^{\prime}, \beta_{2}^{\prime}}(T(M))\right) \cap_{x} \gamma \supset C_{\varepsilon_{2}, \beta_{2}}(T(M)) \cap_{x} \gamma .
$$

(c) Given $\delta$, $\mu$, we choose $z$ sufficiently large so that $C(T(M))-{ }_{\delta / 2} \tau \subset{ }_{z \mu} \gamma$.

(d) Given $\delta, \mu, z, y$, choose $\delta^{\prime}$ sufficiently small so that ${ }_{3 z \mu} \gamma \cap_{2 \delta^{\prime}} \tau=\varnothing$.

Note that it follows from $8.18,8.19,8.21,8.22,8.26,8.28$ that the pieces $\left.g_{t}\right|_{2 \delta^{\prime}} \tau^{\prime \prime}, t \in[0,1]$, and $g_{t} \mid\left(h^{*} C(\xi)-{ }_{2 \delta^{\prime}} \tau^{\prime \prime}\right), t \in[0,1]$, defined by 8.11 and 8.27 , respectively, when unioned together give a well-defined homotopy $g_{t}: h^{*} C(\xi) \rightarrow C(\xi), t \in[0,1]$, that satisfies 8.8 as desired, when $\alpha_{2}$ of $8.8(\mathrm{~d})$ is given by the formula of 8.29 .

8.29. $\alpha_{2}=e^{10 y \mu} \eta^{-3} \Theta+10 y u$.

This completes Step II in the proof of Theorem 5.5.

Step III. In this step, we shall show how to modify the homotopy $g_{t}, t \in[0,1]$, constructed in Step II to get a new homotopy, also denoted by $g_{t}, t \in[0,1]$, that satisfies the following properties.

8.30. Let $\varepsilon, \beta$ denote given positive numbers, and let $f_{t}, t \in[0,1]$, denote the homotopy of 8.2 .

(a) The pair $\left(f_{t}, g_{t}\right), t \in[0,1]$, is an admissible homotopy pair (see Definition 5.3).

(b) Set $(X, \partial X)=\left(g_{1}^{-1}\left({ }_{\delta^{\prime}, \delta} \tau^{\prime}\right), g_{1}^{-1}\left(\partial_{\delta^{\prime}, \delta} \tau^{\prime}\right)\right)$. Then the map $g_{1}:(X, \partial X) \rightarrow$ $\left({ }_{\delta^{\prime}, \delta} \tau^{\prime}, \partial_{\delta^{\prime}, \delta} \tau^{\prime}\right)$ is split over the subcomplex $\partial K_{1, s}^{\prime \prime}$ with respect to the composite projection map

$$
\delta^{\prime}, \delta \stackrel{\tau^{\prime}}{\text { proj }} \rightarrow(\xi) \stackrel{\rho_{D}^{s}}{\rightarrow} S(T(M))
$$

where $\rho_{D}^{s}$ comes from 8.2. Here $K_{1, s}, K_{1, s}^{\prime}$ are the simplical complexes of 8.2 (for $\varepsilon_{1}=\varepsilon, \beta_{1}=\beta$ in 8.2), $\partial K_{1, s}^{\prime}$ is the subcomplex of $K_{1, s}^{\prime}$ that is contained in $S(T(M))$, and $\partial K_{1, s}^{\prime \prime}$ is a subcomplex of $\partial K_{1, s}^{\prime}$ that satisfies

$$
\left|\partial K_{1, s}^{\prime \prime}\right|^{\eta^{-1} \alpha_{3}, \varepsilon} \subset\left|\partial K_{1, s}^{\prime}\right| \subset\left|\partial K_{1, s}^{\prime \prime}\right|^{2 \eta^{-1} \alpha_{3}, 2 \varepsilon} .
$$

(See 8.32(b) for $\alpha_{3}$.) 
(c) The map $g_{1}$ satisfies 8.8(a) and (c), for $\varepsilon_{2}=\varepsilon, \beta_{2}=\beta$ in 8.8(c).

(d) The map $g_{1}: g_{1}^{-1}\left(C(\xi)-{ }_{\delta} \tau^{\prime}\right) \rightarrow C(\xi)-{ }_{\delta} \tau^{\prime}$ is $\alpha$-controlled over all of $M$, where $\alpha$ is a positive number that is independent of $\varepsilon$ and $\beta$. (Here the control data are just ordinary control, not foliated control, and are measured with respect to the projection $C(\xi)-{ }_{\delta} \tau^{\prime} \rightarrow M$.)

(e) The map $g_{1}: g_{1}^{-1}\left({ }_{\delta} \tau^{\prime}\right) \rightarrow{ }_{\delta} \tau^{\prime}$ is $\alpha$-controlled over all of $M$. (Here the control is measured with respect to the projection that is equal to the composite map

$$
{ }_{\delta} \tau^{\prime} \stackrel{\rho_{\tau^{\prime}}}{\rightarrow} S(\xi) \stackrel{\rho_{D}^{s}}{\rightarrow} S(T(M)) \stackrel{\text { proj }}{\rightarrow} M,
$$

where $\rho_{D}^{s}$ comes from 8.2.)

Warning. The homotopy $g_{t}, t \in[0,1]$, of 8.30 will no longer satisfy all of the properties listed in 8.8. However, the homotopy $f_{t}, t \in[0,1]$, of 8.30 will still satisfy all the properties listed in 8.2.

In the remainder of this step, we shall assume that the triangulations $K_{1}$ and $K_{2}$ are related as follows. Let $\partial K_{1}$ denote the subcomplex of $K_{1}$ that triangulates $S(T(M))$.

8.31. For every simplex $\Delta \in \partial K_{1}$, the subset $\rho_{\tau}^{-1}(\Delta) \cap \partial_{\delta^{\prime}, \delta} \tau$ is the underlying set of a subcomplex of $K_{2}$.

Note that it follows from 8.2, 8.8, and 8.31 that the map $g_{1}: h^{*} C(\xi)-$ $h^{*} S(\xi) \rightarrow C(\xi)-S(\xi)$ constructed in Step II is in transverse position to the codimension one submanifold $\partial_{\delta^{\prime}, \delta} \tau^{\prime}$ of $C(\xi)-S(\xi)$. Thus, the pair $X=$ $g_{1}^{-1}\left(\delta^{\prime}, \delta \tau^{\prime}\right), \partial X=g_{1}^{-1}\left(\partial_{\delta^{\prime}, \delta} \tau^{\prime}\right)$ is a manifold pair. It also follows from 8.8, 7.1, and 8.31 that the restricted map $g_{1}:(X, \partial X) \rightarrow\left({ }_{\delta^{\prime}, \delta} \tau^{\prime}, \partial_{\delta^{\prime}, \delta} \tau^{\prime}\right)$ is a homotopy equivalence of manifold pairs that satisfies the following properties.

8.32. (a) The restricted map $g_{1}: \partial X \rightarrow \partial_{\delta^{\prime}, \delta} \tau^{\prime}$ is already split over all of the triangulation $\partial K_{1, s}^{\prime}$ with respect to the bundle projection $\rho_{\tau^{\prime}}^{s}$. Here $K_{1, s}^{\prime}$ comes from 8.2, and $\partial K_{1, s}^{\prime}$ is the subcomplex of $K_{1, s}^{\prime}$ that is contained in $S(T(M)) ; \rho_{\tau^{\prime}}^{s}:{ }_{\delta^{\prime}, \delta} \tau^{\prime} \rightarrow S(T(M))$ denotes the bundle projection that is equal to the composite map

$$
\delta^{\prime}, \delta \tau^{\prime} \stackrel{\rho_{\tau^{\prime}}}{\rightarrow} S(\xi) \stackrel{\rho_{D}^{s}}{\longrightarrow} S(T(M))
$$

where $\rho_{D}^{s}$ comes from 8.2.

(b) The map $g_{1}:(X, \partial X) \rightarrow\left({ }_{\delta^{\prime}, \delta} \tau^{\prime}, \partial_{\delta^{\prime} \delta} \tau^{\prime}\right)$ is $\left(\alpha_{3}, \delta_{3}\right)$-controlled over all of $\left(S(T(M)), \mathscr{G}_{S}\right)$, where $\alpha_{3}$ is a positive number that is independent of $\delta, \delta_{2}$, $s$, and where $\delta_{3}$ is a positive number that satisfies $\lim _{\bar{\delta} \rightarrow 0} \delta_{3}=0$, where $\bar{\delta}=$ $\delta+\delta_{2}+s^{-1}$.

From this point on, the argument of Step III is quite similar to the argument of Step I. So we shall be very brief.

Let $\left\{L_{i}\right\}$ denote all of the closed leaves of $\mathscr{G}_{S}$ that have length less than or equal to $\eta^{-1} \alpha_{3}$, where $\eta$ and $\alpha_{3}$ come from 8.1(a) and 8.32(b). Choose very 
small pairwise disjoint tubular neighborhoods $\left\{T_{i}\right\}$ for the $\left\{L_{i}\right\}$ in $S(T(M))$. There is no loss of generality in assuming that each $T_{i}$ is the underlying set of a finite subcomplex $X_{i}$ of $\partial K_{1, s}$. Choose a maximal subcomplex $\partial K_{1, s}^{\prime \prime}$ of $\partial K_{1, s}^{\prime}$ that satisfies the following: let $\left\{T_{i}: i \in I\right\}$ denote all of the $T_{i}$ that intersect with $\left|\partial K_{1, s}^{\prime \prime}\right|$. Then we must have that

(c) $\left(\left|\partial K_{1, s}^{\prime \prime}\right| \cup\left(\bigcup_{i \in I} T_{i}\right)\right)^{\eta^{-1} \alpha_{3}, \varepsilon} \subset\left|\partial K_{1, s}^{\prime}\right|$.

The data of 8.32 allow us to apply Theorem 6.6 to the map $g_{1} \mid X$ to get a homotopy $g_{t}: X \rightarrow_{\delta^{\prime}, \delta} \tau^{\prime}, t \in[1,2]$, of $g_{1} \mid X$ such that $g_{t} \mid \partial X, t \in[1,2]$, is the constant homotopy and such that $g_{2}:(X, \partial X) \rightarrow\left({ }_{\delta^{\prime}, \delta^{\prime}} \tau^{\prime}, \partial_{\delta^{\prime}, \delta^{\prime}} \tau^{\prime}\right)$ is split over the subcomplex $\partial K_{1, s}^{\prime \prime \prime}$ of $\partial K_{1, s}^{\prime \prime}$, where $\partial K_{1, s}^{\prime \prime \prime}$ is the maximal subcomplex of $\partial K_{1, s}^{\prime \prime}$ that does not intersect $\bigcup_{i \in I}\left(T_{i}-\partial T_{i}\right)$. Next we can apply Theorem 6.2 to $g_{2}$ over each of the subcomplexes $X_{i}, i \in I$, to get a homotopy $g_{t}: X \rightarrow$ $\delta^{\prime}, \delta^{\prime}, t \in[2,3]$, of $g_{2}$ such that $g_{t} \mid \partial X, t \in[2,3]$, is the constant homotopy; $g_{t}, t \in[2,3]$, is the constant homotopy on the preimage of the set $S(T(M))-$ $\bigcup_{i \in I} T_{i}$ (the preimage under the projection $\rho_{\tau^{\prime}}^{s}$ ); $g_{3}$ is split over each of the subcomplexes $X_{i}, i \in I$.

We summarize the results of the preceding paragraph.

8.33. (a) The homotopy $g_{t}: X \rightarrow{ }_{\delta^{\prime}, \delta} \tau^{\prime}, t \in[1,3]$, has compact support. The restricted homotopy $g_{t} \mid \partial X, t \in[1,3]$, is the constant homotopy.

(b) The map $g_{3}:(X, \partial X) \rightarrow\left({ }_{\delta^{\prime}, \delta} \tau^{\prime}, \partial_{\delta^{\prime}, \delta^{\prime}} \tau^{\prime}\right)$ is split over the subcomplex $\partial K_{1, s}^{\prime \prime}$ with respect to the projection $\rho_{\tau^{\prime}}^{s}:_{\delta^{\prime}, \delta} \tau^{\prime} \rightarrow S(T(M))$.

(c) The homotopy $g_{t}, t \in[1,3]$, is $\left(\eta^{-1} \alpha_{3}, \delta_{3}^{\prime}\right)$-controlled over $(S(T(M))$, $\mathscr{G}_{S}$ ), where $\delta_{3}^{\prime}$ is a positive number satisfying $\lim _{\delta_{3} \rightarrow 0} \delta_{3}^{\prime}=0$. (Here the foliated control data is measured with respect to the projection $\rho_{\tau^{\prime}}^{s}: \delta^{\prime}, \delta^{\prime} \rightarrow S(T(M))$.)

Now define homotopies $\bar{f}_{t}: h^{*} D(\xi) \rightarrow D(\xi), t \in[0,1]$, and $\bar{g}_{t}: h^{*} C(\xi) \rightarrow$ $C(\xi), t \in[0,1]$, as follows.

8.34. (a)

$$
\bar{f}_{t}(x)= \begin{cases}f_{2 t}(x), & \text { if } t \in\left[0, \frac{1}{2}\right] \\ f_{1}(x), & \text { if } t \in\left[\frac{1}{2}, 1\right]\end{cases}
$$

(b)

$$
\bar{g}_{t}(x)= \begin{cases}g_{2 t}(x), & \text { if } t \in\left[0, \frac{1}{2}\right], \\ g_{1}(x), & \text { if } t \in\left[\frac{1}{2}, 1\right] \text { and } x \notin X, \\ g_{t^{\prime}}(x), & \text { if } t \in\left[\frac{1}{2}, 1\right] \text { and } x \in X, \text { where } t^{\prime}=4\left(t-\frac{1}{2}\right)+1 .\end{cases}
$$

Note that the homotopy $\bar{f}_{t}, t \in[0,1]$, defined by 8.34 satisfies 8.2 (when $\bar{f}_{t}$ replaces $f_{t}$ in 8.2$)$. Note that it follows from 8.1(b), 8.31, 8.8, 8.33, 8.34 that the pair $\left(\bar{f}_{t}, \bar{g}_{t}\right), t \in[0,1]$, satisfy 8.30 -when in 8.30 we replace $\left(f_{t}, g_{t}\right)$ by $\left(\bar{f}_{t}, \bar{g}_{t}\right)$-provided $\varepsilon_{1}, \varepsilon_{2}$ of 8.2 and 8.8 are choosen sufficiently small and provided the $\beta_{1}, \beta_{2}$ of 8.2 and 8.8 are choosen sufficiently large.

This completes Step III. 
Step IV. In this step, we complete the proof of Theorem 5.5 in the special case that the fiber of the fiber bundle $p: E \rightarrow M$ has no boundary.

Recall that $M$ is equal to the topological product $M^{\prime} \times R$ equipped with the metric of (3.8.4), where $M^{\prime}$ is another complete real hyperbolic manifold. Let $\bar{M}$ denote the space $M^{\prime} \times R$ equipped with the product metric, and let $\bar{r}: M \rightarrow \bar{M}$ denote the topological identification $M=M^{\prime} \times R$. Note that it follows from Remark 3.5.1 that $\bar{r}: M \rightarrow \bar{M}$ satisfies the following property.

8.35. Let $\varepsilon, \beta$ be the numbers given in 8.30. Then for any $v \in$ $\operatorname{Tan}\left(M-M_{0, \beta}\right)$, we have that $\langle d \bar{r}(v), d \bar{r}(v)\rangle_{\bar{M}} \leq \beta^{-1}\langle v, v\rangle_{M}$ (for sufficiently large $\beta)$.

Set $\bar{M}_{\varepsilon, \beta}=\bar{r}\left(M_{\varepsilon, \beta}\right)$.

In this step, we shall need projection maps $P_{C}^{s}: C(\xi) \rightarrow \bar{M}, P_{D}^{s}: D(\xi) \rightarrow \bar{M}$, $q_{C}^{s}: C(T(M)) \rightarrow \bar{M}$, and $q_{D}^{s}: D(T(M)) \rightarrow \bar{M}$ defined as follows.

8.36. (a) $q_{D}^{s}$ is equal to the composite map

$$
D(T(M)) \stackrel{g^{s}}{\rightarrow} D(T(M)) \stackrel{\text { proj }}{\rightarrow} M \stackrel{\bar{r}}{\rightarrow} \bar{M},
$$

where $g^{t}: D(T(M)) \rightarrow D(T(M)), t \in(-\infty,+\infty)$, is the geodesic flow on $D(T(M))$ and where $s>0$ comes from 8.2.

(b) $P_{D}^{S}$ is equal to the composite map

$$
D(\xi) \stackrel{\text { proj }}{\longrightarrow} D(T(M)) \stackrel{q_{D}^{s}}{\longrightarrow} \bar{M}
$$

(c) $\left.q_{C}^{s}\right|_{\delta} \tau$ is equal to the composite map

$$
{ }_{\delta} \tau \stackrel{\text { proj }}{\rightarrow} S(T(M)) \stackrel{q_{D}^{s}}{\longrightarrow} \bar{M} .
$$

Set $q_{C}^{s} \mid\left(C(T(M))-{ }_{2 \delta} \tau\right)=\bar{r} \circ$ proj. Finally, for each $t \in[0,1]$, set $\left.q_{C}^{s}\right|_{(1+t) \delta} \dot{\tau}$ equal to the composite map

$$
(1+t) \delta \dot{\tau} \stackrel{\text { proj }}{\rightarrow} S(T(M)) \stackrel{q_{D}^{(1-t) s}}{\rightarrow} \bar{M},
$$

where $q_{D}^{(1-t) s}$ is defined as in (a) when $s$ is replaced by $(1-t) s$.

(d) $P_{C}^{s}$ is equal to the composite map

$$
C(\xi) \stackrel{\text { proj }}{\longrightarrow} C(T(M)) \stackrel{q_{C}^{s}}{\rightarrow} \bar{M} .
$$

Let $\bar{K}$ denote the triangulation for $\bar{M}$ that is the image of the triangulation $K$ under $\bar{r}: M \rightarrow \bar{M}$. In this step, we shall assume that the following relations hold between the triangulations $\bar{K}, K_{1}, K_{2}$ of $\bar{M}, D(T(M))$, $C(T(M))-S(T(M))$.

(e) For each simplex $\Delta \in \bar{K}$, the sets $\left(q_{D}^{s}\right)^{-1}(\Delta)$ and $\left(q_{C}^{s}\right)^{-1}(\Delta) \cap X$ are the underlying sets of subcomplexes of $K_{1}$ and of $K_{2}$, where $X={ }_{\delta .2 \delta} \tau,{ }_{\delta} \dot{\tau},{ }_{2 \delta} \dot{\tau}$, or $C(T(M))-S(T(M))$.

Note that by 8.2 and 8.36 we have that $f_{1}: h^{*} D(\xi) \rightarrow D(\xi)$ is split over the subcomplex $\bar{K}^{\prime}$ of $\bar{K}$, where $\bar{K}^{\prime}$ is the image under $\bar{r}: M \rightarrow \bar{M}$ of the 
subcomplex $K^{\prime}$ of $K$, where $K^{\prime}$ is the minimal subcomplex of $K$ containing $M_{\varepsilon, \beta}$. (Here the splitting is measured with respect to the projection $P_{D}^{s}$.) It follows from 8.2 and 8.35 that $f_{1}$ is $2 \beta^{-1}\left(\eta^{-1} \Theta+\delta_{1}\right)$-controlled over the subset $\bar{M}-M^{\prime} \times(-\beta / 2, \beta / 2)$ of $\bar{M}$. (Here the control is measured with respect to the projection $P_{D}^{s}$.) Finally, we note that there is an $\varepsilon^{\prime}>0$, depending only on $\varepsilon$ and $\operatorname{dim}(M)$, such that the foliation of $\bar{M}$ by its points is uniformly $\left(\varepsilon^{\prime}, \sigma\right)$-flat on $M^{\prime^{\varepsilon}} \times R$, where $\sigma$ is the number given by Theorem 6.6 for $\bar{M}$ and its pointwise foliation (see Lemma 3.15). Thus, if we choose $\beta>$ $2\left(\varepsilon^{\prime}\right)^{-1}\left(\eta^{-2} \boldsymbol{\Theta}+\eta^{-1} \delta_{1}\right)$, we may apply Theorem 6.6 to $f_{1}$ to get a splitting for $f_{1}$ over the minimal subcomplex of $\bar{K}$ that contains the subset $M^{2^{2 \varepsilon}} \times R$. Now if $\varepsilon$ is sufficiently small, we have that $f_{1}$ is split over all of $\bar{K}$ except in tubular neighborhoods of the cusps of $M^{\prime} \times 0$ and of the short geodesic of $M^{\prime} \times 0$. Since these tubular neighborhoods are disc bundles over compact closed flat manifolds, we now may use Theorem 6.2 to extend the splitting of $f_{1}$ over all of $\bar{K}$. (All of the above splittings are measured with respect to the projection $P_{D}^{S}$.) We can summarize the results obtained in this paragraph as follows. There is a homotopy $f_{t}:\left(h^{*} D(\xi), h^{*} S(\xi)\right) \rightarrow(D(\xi), S(\xi)), t \in[1,2]$, of $f_{1}$ that satisfies the following properties.

8.37. (a) The homotopy $f_{t}, t \in[1,2]$, has compact support.

(b) The homotopy $f_{t}, t \in[1,2]$, is $2 \beta^{-1}\left(\eta^{-2} \Theta+\eta^{-1} \delta_{1}\right)$-controlled over the subset $\bar{M}_{2 \varepsilon, \infty}-\bar{M}_{2 \varepsilon, \beta / 2}$ with respect to the projection $P_{D}^{s}$.

(c) The map $f_{2}:\left(h^{*} D(\xi), h^{*} S(\xi)\right) \rightarrow(D(\xi), S(\xi))$ is split over the entire triangulation $\bar{K}$ with respect to the projection $P_{D}^{s}$.

(d) The homotopy $f_{t} \mid Y, t \in[1,2]$, is the constant homotopy, where $Y$ is the preimage of the set $\bar{M}_{2 \varepsilon, \beta / 2}$ under the projection $P_{D}^{s}$.

Note that $8.30(\mathrm{c})$ allows us to use the argument given in the first part of Step II to "lift" the homotopy $f_{t} \mid h^{*} S(\xi), t \in[1,2]$, to a homotopy $g_{t}:{ }_{\delta^{\prime}} \tau^{\prime \prime} \rightarrow{ }_{\delta^{\prime}} \tau^{\prime}$, $t \in[1,2]$, of $g_{1}$ that satisfies the following properties.

8.38. (a) For each $x \in h^{*} S(\xi)$ and each $t \in[1,2]$, we have that $g_{t}$ maps $\delta_{\delta^{\prime} / 2} \tau_{x}^{\prime \prime}$ homeomorphically onto ${ }_{\delta^{\prime} / 2} \tau_{f_{t}(x)}^{\prime}$.

(b) $\left.g_{t}\right|_{\delta^{\prime}} \dot{\tau}^{\prime \prime}, t \in[1,2]$, is the constant homotopy.

(c) The homotopy $\left.g_{t}\right|_{\delta^{\prime}} \tau^{\prime \prime}, t \in[1,2]$, has compact support.

(d) The homotopy $g_{t} \mid Y^{\prime} \cap{ }_{\delta^{\prime}} \tau^{\prime \prime}, t \in[1,2]$, is the constant homotopy, where $Y^{\prime}$ is the preimage of the set $\bar{M}_{2 \varepsilon, \beta / 2}$ under the projection $P_{C}^{s}$.

(e) The homotopy $\left.g_{t}\right|_{\delta^{\prime}} \tau^{\prime \prime}, t \in[1,2]$, is $2^{3} \beta^{-1}\left(\eta^{-2} \Theta+\eta^{-1} \delta_{1}\right)$-controlled over the subset $\bar{M}_{2 \varepsilon, \infty}-\bar{M}_{2 \varepsilon, \beta / 2}$. (Here control is measured with respect to the projection $P_{C}^{s}$.)

We extend the homotopy $g_{t}:{ }_{\delta^{\prime}} \tau^{\prime \prime} \rightarrow{ }_{\delta^{\prime}} \tau^{\prime}, t \in[1,2]$, to a homotopy $g_{t}$ : $g_{1}^{-1}\left({ }_{\delta} \tau^{\prime}\right) \rightarrow{ }_{\delta} \tau^{\prime}, t \in[1,2]$, of $g_{1} \mid g_{1}^{-1}\left({ }_{\delta} \tau^{\prime}\right)$ by letting $g_{t} \mid g_{1}^{-1}\left({ }_{\delta^{\prime}, \delta} \tau^{\prime}\right), t \in[1,2]$, be the constant homotopy. Note that it follows from $8.37(\mathrm{c}), 8.38(\mathrm{a}), 8.30(\mathrm{c})$ 
that

$$
g_{2}:\left(g_{1}^{-1}\left(_{\delta^{\prime} / 2, \delta} \tau^{\prime}\right), g_{1}^{-1}\left(\partial_{\delta^{\prime} / 2, \delta} \tau^{\prime}\right)\right) \rightarrow\left({ }_{\delta^{\prime} / 2, \delta} \tau^{\prime}, \partial_{\delta^{\prime} / 2, \delta} \tau^{\prime}\right)
$$

is a homotopy equivalence of manifold pairs that has compact support and for which $g_{2}: g_{1}^{-1}\left(\partial_{\delta^{\prime} / 2, \delta} \tau^{\prime}\right) \rightarrow \partial_{\delta^{\prime} / 2, \delta} \tau^{\prime}$ is split over the entire triangulation $\bar{K}$ with respect to the projection $P_{C}^{s} \mid \partial_{\delta^{\prime} / 2, \delta} \tau^{\prime}$. Moreover, it follows from 8.38(d), (e), 8.30(b), (e), 8.35, and 8.36 that this homotopy equivalence is $\alpha^{\prime}$-controlled over the subset $\bar{M}_{2 \varepsilon, \infty}-\bar{M}_{2 \varepsilon, \beta / 2}$ of $\bar{M}$, where $\alpha^{\prime}=2^{3} \beta^{-1}\left(\eta^{-2} \Theta+\eta^{-1} \delta_{1}\right)$ $+\beta^{-1} \alpha$, and is split over the minimal subcomplex of $\bar{K}$ that contains the subset $\bar{M}_{2 \varepsilon, \beta / 2}$ of $\bar{M}$. (Here the splitting and the control are measured with respect to the projection $\left.P_{C}^{s}\right|_{\delta^{\prime} / 2, \delta} \tau^{\prime}$.) Finally, we recall that the foliation of $\bar{M}$ by its points is uniformly $\left(\varepsilon^{\prime}, \sigma\right)$-flat on the subset $M^{\prime^{\varepsilon}} \times R$ of $\bar{M}$. Thus, if we choose $\beta>\left[2^{3}\left(\eta^{-3} \boldsymbol{\theta}+\eta^{-2} \delta_{1}\right)+\eta^{-1} \alpha\right]\left(\varepsilon^{\prime}\right)^{-1}$, then we may apply Theorem 6.6 to $g_{2}: g_{1}^{-1}\left({ }_{\delta^{\prime} / 2, \delta} \tau^{\prime}\right) \rightarrow{ }_{\delta^{\prime} / 2, \delta} \tau^{\prime}$ to get a splitting for $g_{2}$ over the minimal subcomplex of $\bar{K}$ that contains the subset $M^{\prime^{3 \varepsilon}} \times R$ of $\bar{M}$, which extends the splitting of $g_{2} \mid g_{1}^{-1}\left(\partial_{\delta^{\prime} / 2, \delta} \tau^{\prime}\right)$ over all of $\bar{K}$. Now if $\varepsilon$ is chosen small enough, we will have that the map $g_{2} \mid g_{1}^{-1}\left({ }_{\delta^{\prime} / 2, \delta} \tau^{\prime}\right)$ is split over all of $\bar{K}$ except in tubular neighborhoods of the cusps of $M^{\prime} \times 0$ and of short geodesic of $M^{\prime} \times 0$. So we can use Theorem 6.2 to extend the splitting of $g_{2} \mid g_{1}^{-1}\left(\delta_{\delta^{\prime} / 2, \delta} \tau^{\prime}\right)$ over all of $\bar{K}$. (All the above splittings are measured with respect to the projection $\left.P_{C}^{s}\right|_{\delta^{\prime} / 2, \delta} \tau^{\prime}$.) We can summarize the results obtained in this paragraph as follows. There is a homotopy $g_{t}:\left(g_{1}^{-1}\left({ }_{\delta^{\prime} / 2, \delta} \tau^{\prime}\right), g_{1}^{-1}\left(\partial_{\delta^{\prime} / 2, \delta} \tau^{\prime}\right)\right) \rightarrow\left({ }_{\delta^{\prime} / 2, \delta} \tau^{\prime}, \partial_{\delta^{\prime} / 2, \delta} \tau^{\prime}\right), t \in[1,3]$, of the map $g_{1} \mid g_{1}^{-1}\left({ }_{\delta^{\prime} / 2, \delta} \tau^{\prime}\right)$ that satisfies the following properties.

8.39. (a) The homotopy $g_{t} \mid g_{1}^{-1}\left({ }_{\delta^{\prime} / 2, \delta} \tau^{\prime}\right), t \in[1,3]$, has compact support.

(b) The restricted homotopies $g_{t} \mid g_{1}^{-1}\left({ }_{\delta} \dot{\tau}^{\prime}\right), t \in[1,3]$, and $g_{t} \mid g_{1}^{-1}\left({ }_{\delta^{\prime} / 2} \dot{\tau}^{\prime}\right)$, $t \in[2,3]$, are the constant homotopies; the homotopy $g_{t} \mid g_{1}^{-1}\left({ }_{\delta^{\prime} / 2} \dot{\tau}^{\prime}\right), t \in$ $[1,2]$, is as in $8.38(\mathrm{a})$.

(c) The map $g_{3}:\left(g_{1}^{-1}\left({ }_{\delta^{\prime} / 2, \delta} \tau^{\prime}\right), g_{1}^{-1}\left(\partial_{\delta^{\prime} / 2, \delta} \tau^{\prime}\right)\right) \rightarrow\left({ }_{\delta^{\prime} / 2, \delta} \tau^{\prime}, \partial_{\delta^{\prime} / 2, \delta} \tau^{\prime}\right)$ is split over the entire triangulation $\bar{K}$ with respect to the projection $\left.P_{C}^{s}\right|_{\delta^{\prime} / 2, \delta} \tau^{\prime}$.

We finally consider the homotopy equivalence

$$
g_{1}:\left(g_{1}^{-1}\left(\overline{C(\xi)-{ }_{\delta} \tau^{\prime}}\right), g_{1}^{-1}\left({ }_{\delta} \dot{\tau}^{\prime}\right)\right) \rightarrow\left(\overline{C(\xi)-{ }_{\delta} \tau^{\prime}},{ }_{\delta} \dot{\tau}^{\prime}\right)
$$

between manifold pairs. It follows from $8.36,8.30(\mathrm{c})$, and $8.8(\mathrm{c})$ that $g_{1}$ : $g_{1}^{-1}\left(\delta_{\delta, 2 \delta} \tau^{\prime}\right) \rightarrow_{\delta, 2 \delta} \tau^{\prime}$ is already split over the entire triangulation $\bar{K}$ with respect to the projection $\left(\left.P_{C}^{S}\right|_{\delta, 2 \delta} \tau^{\prime}\right):_{\delta, 2 \delta} \tau^{\prime} \rightarrow \bar{M}$. It follows from $8.30(\mathrm{c}), 8.8(\mathrm{c})$, and 8.36 that the map $g_{1}: g_{1}^{-1}\left(\overline{C(\xi)-{ }_{2 \delta} \tau^{\prime}}\right) \rightarrow \overline{C(\xi)-{ }_{2} \tau^{\prime}}$ is split over the subcomplex $\bar{K}^{\prime}$ of $\bar{K}$ with respect to the projection $P_{C}^{s}$. (Here $\bar{K}^{\prime}$ is the minimal subcomplex of $\bar{K}$ that contains $\bar{M}_{\varepsilon, \beta}$.) By 8.36 and $8.30(\mathrm{~d})$, we have that the map $g_{1}: g_{1}^{-1}\left(\overline{C(\xi)-{ }_{2 \delta} \tau^{\prime}}\right) \rightarrow \overline{C(\xi)-{ }_{2 \delta} \tau^{\prime}}$ is $\beta^{-1} \alpha$-controlled over the subset $\bar{M}_{\varepsilon, \infty}-\bar{M}_{\varepsilon, \beta}$ with respect to the projection $P_{C}^{s}$. Since the foliation 
of $\bar{M}$ by its points is uniformly $\left(\varepsilon^{\prime}, \sigma\right)$-flat on the subset $M_{\varepsilon}^{\prime} \times R$ of $\bar{M}$, we may, provided $\beta$ is choosen to satisfy $\beta>\eta^{-1}\left(\varepsilon^{\prime}\right)^{-1} \alpha$, apply Theorem 6.6 to $g_{1} \mid g_{1}^{-1}\left(\overline{C(\xi)-{ }_{2 \delta} \tau^{\prime}}\right)$ over $M^{t^{\varepsilon}} \times R$ to get a splitting for this map over the minimal subcomplex of $\bar{K}$ that contains the subset $M^{{ }^{2 e}} \times R$ of $\bar{M}$ (this splitting is also with respect to the projection $\left.P_{C}^{s}\right)$. Now the map $g_{1} \mid g_{1}^{-1}\left(\overline{C(\xi)-{ }_{2 \delta} \tau^{\prime}}\right)$ is split over the entire triangulation $\bar{K}$ except in tubular neighborhoods of the cusps of $M^{\prime} \times 0$ and of short geodesics of $M^{\prime} \times 0$. So we may again apply Theorem 6.2 to extend the splitting for this map over these tubular neighborhoods. We can summarize the results of this paragraph as follows. There is a homotopy $g_{t}: g_{1}^{-1}\left(\overline{C(\xi)-{ }_{\delta} \tau^{\prime}}\right) \rightarrow \overline{C(\xi)-{ }_{\delta} \tau^{\prime}}, t \in[1,3]$, of the map $g_{1} \mid g_{1}^{-1}\left(\overline{C(\xi)-{ }_{\delta} \tau^{\prime}}\right)$ that satisfies the following properties.

8.40. (a) The homotopy $g_{t} \mid g_{1}^{-1}\left(\overline{C(\xi)-{ }_{\delta} \tau^{\prime}}\right), t \in[1,3]$, has compact support.

(b) The restricted homotopy $g_{t} \mid g_{1}^{-1}\left({ }_{\delta} \dot{\tau}^{\prime}\right), t \in[1,3]$, is the constant homotopy.

(c) The map $g_{3}:\left(g_{1}^{-1}\left(\overline{C(\xi)-{ }_{\delta} \tau^{\prime}}\right), g_{1}^{-1}\left({ }_{\delta} \dot{\tau}^{\prime}\right)\right) \rightarrow\left(\overline{C(\xi)-{ }_{\delta} \tau^{\prime}},{ }_{2 \delta} \dot{\tau}^{\prime}\right)$ is split over the entire triangulation $\bar{K}$ with respect to the projection $P_{C}^{s}$.

We extend the homotopy $f_{t}, t \in[0,2]$, of 8.2 and 8.37 to a homotopy $f_{t}: h^{*} D(\xi) \rightarrow D(\xi), t \in[0,3]$, by letting $f_{t}, t \in[2,3]$, be the constant homotopy. We extend the homotopy $g_{t}::_{\delta^{\prime} / 2} \tau^{\prime \prime} \rightarrow{ }_{\delta^{\prime} / 2} \tau^{\prime}, t \rightarrow[0,2]$, given by 8.30 and 8.38 to a homotopy $g_{t}:{ }_{\delta^{\prime} / 2} \tau^{\prime \prime} \rightarrow{ }_{\delta^{\prime} / 2} \tau^{\prime}, t \in[0,3]$, by letting $\left.g_{t}\right|_{\delta^{\prime} / 2} \tau^{\prime \prime}$, $t \in[2,3]$, be the constant homotopy. Then these trivial extensions, together with the homotopy $g_{t}: \overline{h^{*} C(\xi)-{ }_{\delta^{\prime} / 2} \tau^{\prime \prime}} \rightarrow \overline{C(\xi)-{ }_{\delta^{\prime} / 2} \tau^{\prime}}, t \in[0,3]$, given by 8.30, 8.39, and 8.40, give an admissible homotopy pair $\left(f_{t}, g_{t}\right), t \in[0,3]$. Define $f_{t}, g_{t}, t \in[3,4]$, by $f_{t}=h_{t-3}^{s} \circ f_{3}, g_{t}=H_{t-3}^{s} \circ g_{3}$, where $h_{u}^{s}: D(\xi) \rightarrow$ $D(\xi), u \in[0,1]$, and $H_{u}^{s}: C(\xi) \rightarrow C(\xi), u \in[0,1]$, are isotopies that satisfy the following properties. (It is left to the reader to construct such isotopies.)

8.41. (a) The pair $\left(h_{u}^{s}, H_{u}^{s}\right), u \in[0,1]$, is an admissable homotopy pair (see Definition 5.3).

(b) $h_{0}^{s}, H_{0}^{s}$ are both identity maps.

(c) We have that $P_{D}^{s}=Q_{D} \circ h_{1}^{s}$ and $P_{C}^{s}=Q_{C} \circ H_{1}^{s}$, where $Q_{D}$ is the composite $D(\xi) \stackrel{\text { proj }}{\rightarrow} M \stackrel{\bar{r}}{\rightarrow} \bar{M}$ and $Q_{C}$ is the composite $C(\xi) \stackrel{\text { proj }}{\rightarrow} M \stackrel{\bar{r}}{\rightarrow} \bar{M}$.

Finally, set $\bar{f}_{t}=f_{4 t}, \bar{g}_{t}=g_{4 t}$ for all $t \in[0,1]$. Then the pair $\left(\bar{f}_{t}, \bar{g}_{t}\right)$, $t \in[0,1]$, satisfies the conclusions of Theorem 5.5.

This completes the proof of Theorem 5.5.

\section{COMPUTATION OF SURGERY GROUPS}

Let $p: E \rightarrow M$ denote a fiber bundle over the complete real hyperbolic manifold $M$ having the compact manifold $F$ for fiber. Let $w: \pi_{1}(E) \rightarrow Z_{2}$ be the homomorphism obtained by capping with the first Stiefel-Whitney class of $E$, and let $w^{\prime}: \pi_{1}(F) \rightarrow Z_{2}$ denote the homomorphism obtained by capping with the first Stiefel-Whitney class of $F$. In this section, we show that the 
surgery groups $L_{k}^{-\infty}\left(\pi_{1}(E), w\right)$ are isomorphic to cohomology groups of $M$ (with compact support) having for twisted coefficients the surgery classifying spaces $\mathbf{L}_{l}^{-\infty}\left(\pi_{1}(F), w^{\prime}\right)$ (with the twisting induced by the transition functions of the bundle $p: E \rightarrow M)$. Recall that the surgery groups $L_{l}^{-\infty}($,$) are defined$ by Ranicki [60]. We denote by $\mathbf{L}_{l}^{-\infty}\left(\pi_{1}(F), w^{\prime}\right)$ the surgery classifying space that has the surgery groups $L_{l+*}^{-\infty}\left(\pi_{1}(F), w^{\prime}\right)$ for homotopy groups. For details about surgery classifying spaces, see Quinn [57, 58], Jones [39, 40], and Nicas [52].

By killing the higher homotopy groups of the fibers of $p: E \rightarrow M$, we obtain a homotopy fibration $p^{\prime}: E^{\prime} \rightarrow M$ and a map $r: E \rightarrow E^{\prime}$ satisfying the following properties.

9.1. (a) $p^{\prime} \circ r=p$.

(b) Each fiber of $p^{\prime}$ is a $K(\pi, 1)$-space.

(c) For each $x \in M$ the restricted map $r: p^{-1}(x) \rightarrow\left(p^{\prime}\right)^{-1}(x)$ is 2-connected.

9.2. The cohomology groups $H_{c}^{q}\left(M ; L^{h}(p)\right)$. Let $T$ be a piecewise smooth triangulation for $M$, and let $K_{1}, K_{2}$ denote subcomplexes of $T$ with $K_{2} \subset$ $K_{1}$. For each integer $q \geq 5$, we define a group $H_{c}^{q}\left(K_{1}, K_{2} ; L^{h}(p)\right)$ as follows. Any element in $H_{c}^{q}\left(K_{1}, K_{2} ; L^{h}(p)\right)$ is represented by a pair of maps $f: X \rightarrow$ $Y, g: Y \rightarrow E^{\prime}$ satisfying (a)-(d) below. Two different pairs of maps $f: X \rightarrow$ $Y, g: Y \rightarrow E^{\prime}$ and $f^{\prime}: X^{\prime} \rightarrow Y^{\prime}, g^{\prime}: Y^{\prime} \rightarrow E^{\prime}$ represent the same element in $H_{c}^{q}\left(K_{1}, K_{2} ; L^{h}(p)\right)$ if there is a third pair of maps $\bar{f}: \bar{X} \rightarrow \bar{Y}, \bar{g}: \bar{Y} \rightarrow E^{\prime}$ that satisfies $(\mathrm{e})-(\mathrm{g})$ below.

(a) The map $f: X \rightarrow Y$ has compact support (see Remark 4.3).

(b) For each simplex $\Delta \in K_{1}$ define 4-tuples

$$
\left(X(\Delta), \partial_{1} X(\Delta), \partial_{2} X(\Delta), \wedge X(\Delta)\right) \text { and }\left(Y(\Delta), \partial_{1} Y(\Delta), \partial_{2} Y(\Delta), \wedge Y(\Delta)\right)
$$

as follows:

$$
\begin{array}{cc}
Y(\Delta)=\left(p^{\prime} \circ g\right)^{-1}(\Delta) ; & \partial_{1} Y(\Delta)=\bigcup_{\Delta^{\prime} \in \partial \Delta} Y\left(\Delta^{\prime}\right) ; \\
\partial_{2} Y(\Delta)=\overline{\partial Y(\Delta)-\partial_{1} Y(\Delta)} ; & \wedge Y(\Delta)=\partial_{1} Y(\Delta) \cap \partial_{2} Y(\Delta) ; \\
X(\Delta)=\left(p^{\prime} \circ g \circ f\right)^{-1}(\Delta) ; & \partial_{1} X(\Delta)=\bigcup_{\Delta^{\prime} \in \partial \Delta} X\left(\Delta^{\prime}\right) ; \\
\partial_{2} X(\Delta)=\overline{\partial X(\Delta)-\partial_{1} X(\Delta) ;} & \wedge X(\Delta)=\partial_{1} X(\Delta) \cap \partial_{2} X(\Delta) .
\end{array}
$$

We require that each $X(\Delta), Y(\Delta)$ be a compact manifold of dimension equal to $q+\operatorname{dim}(\Delta)$ - the manifold boundaries of $X(\Delta), Y(\Delta)$ are denoted by $\partial X(\Delta)$, $\partial Y(\Delta)$-and we also require that each of $\partial_{1} X(\Delta), \partial_{1} Y(\Delta)$ be a compact codimension zero submanifold of $\partial X(\Delta), \partial Y(\Delta)$ having $\wedge X(\Delta), \wedge Y(\Delta)$ for manifold boundary, respectively. 
(c) For each $\Delta \in K_{1}$, the map $f:\left(X(\Delta), \partial_{1} X(\Delta), \partial_{2} X(\Delta), \wedge X(\Delta)\right) \rightarrow$ $\left(Y(\Delta), \partial_{1} Y(\Delta), \partial_{2} Y(\Delta), \wedge Y(\Delta)\right)$ is a degree one normal map between manifold 4-tuples each of whose orientable covers are equipped with an orientation. Moreover, the orientation data and the normal data (i.e., the framing information) are compatible with inclusions $\Delta^{\prime} \subset \Delta$ for all $\Delta^{\prime}, \Delta \in K_{1}$.

(d) For each $\Delta \in K_{1}$, the map $f: \partial_{2} X(\Delta) \rightarrow \partial_{2} Y(\Delta)$ is a homotopy equivalence. For each $\Delta \in K_{2}$, the map $f: X(\Delta) \rightarrow Y(\Delta)$ is a homotopy equivalence.

(e) For each $\Delta \in K_{1}$, set $\bar{Y}(\Delta)=\left(p^{\prime} \circ \bar{g}\right)^{-1}(\Delta)$ and set $\bar{X}(\Delta)=$ $\left(p^{\prime} \circ \bar{g} \circ \bar{f}\right)^{-1}(\Delta)$. We require that $\bar{X}(\Delta), \bar{Y}(\Delta)$ be compact cobordisms from $X(\Delta), Y(\Delta)$ to $X^{\prime}(\Delta), Y^{\prime}(\Delta)$.

(f) For each $\Delta \in K_{1}$, define 4-tuples $\left(\bar{Y}(\Delta), \partial_{1} \bar{Y}(\Delta), \partial_{2} \bar{Y}(\Delta), \wedge \bar{Y}(\Delta)\right)$ and $\left(\bar{X}(\Delta), \partial_{1} \bar{X}(\Delta), \partial_{2} \bar{X}(\Delta), \wedge \bar{X}(\Delta)\right)$ as follows:

$$
\begin{aligned}
& \partial_{1} \bar{Y}(\Delta)=\bigcup_{\Delta^{\prime} \in \partial \Delta} \bar{Y}\left(\Delta^{\prime}\right) \\
& \partial_{2} \bar{Y}(\Delta)=\overline{\partial \bar{Y}(\Delta)-\partial_{1} \bar{Y}(\Delta)-Y(\Delta)-Y^{\prime}(\Delta)} ; \quad \wedge \bar{Y}(\Delta)=\partial_{1} \bar{Y}(\Delta) \cap \partial_{2} \bar{Y}(\Delta) ; \\
& \partial_{1} \bar{X}(\Delta)=\bigcup_{\Delta^{\prime} \in \partial \Delta} \bar{X}\left(\Delta^{\prime}\right) ; \quad \partial_{2} \bar{X}(\Delta)=\overline{\bar{X}(\Delta)-\partial_{1} \bar{X}(\Delta)-X(\Delta)-X^{\prime}(\Delta)} ; \\
& \wedge \bar{X}(\Delta)=\partial_{1} \bar{X}(\Delta) \cap \partial_{2} \bar{X}(\Delta) .
\end{aligned}
$$

We require that each map

$$
\bar{f}:\left(\bar{X}(\Delta), \partial_{1} \bar{X}(\Delta), \partial_{2} \bar{X}(\Delta), \wedge \bar{X}(\Delta)\right) \rightarrow\left(\bar{Y}(\Delta), \partial_{1} \bar{Y}(\Delta), \partial_{2} \bar{Y}(\Delta), \wedge \bar{Y}(\Delta)\right)
$$

be a degree one normal cobordism from

$$
f:\left(X(\Delta), \partial_{1} X(\Delta), \partial_{2} X(\Delta), \wedge X(\Delta)\right) \rightarrow\left(Y(\Delta), \partial_{1} Y(\Delta), \partial_{2} Y(\Delta), \wedge Y(\Delta)\right)
$$

to

$$
f^{\prime}:\left(X^{\prime}(\Delta), \partial_{1} X^{\prime}(\Delta), \partial_{2} X^{\prime}(\Delta), \wedge X^{\prime}(\Delta)\right) \rightarrow\left(Y^{\prime}(\Delta), \partial_{1} Y^{\prime}(\Delta), \partial_{2} Y^{\prime}(\Delta), \wedge Y^{\prime}(\Delta)\right) \text {. }
$$

Moreover, the orientation data and the normal data of these normal cobordisms must be compatible when the inclusions $\Delta^{\prime} \subset \Delta$ for all $\Delta^{\prime}, \Delta \in K_{1}$.

(g) For each $\Delta \in K_{1}$, the map $\bar{f}: \partial_{2} \bar{X}(\Delta) \rightarrow \partial_{2} \bar{Y}(\Delta)$ is a homotopy equivalence. For each $\Delta \in K_{2}$, the map $\bar{f}: \bar{X}(\Delta) \rightarrow \bar{Y}(\Delta)$ is a homotopy equivalence.

For any elements $\alpha, \alpha^{\prime} \in H_{c}^{q}\left(K_{1}, K_{2}: L^{h}(p)\right)$ represented by pairs of maps $f: X \rightarrow Y, g: Y \rightarrow E^{\prime}$ and $f^{\prime}: X^{\prime} \rightarrow Y^{\prime}, g: Y^{\prime} \rightarrow E^{\prime}$ the sum $\alpha+\alpha^{\prime}$ is represented by the pair of maps $f^{\prime \prime}: X^{\prime \prime} \rightarrow Y^{\prime \prime}, g^{\prime \prime}: Y^{\prime \prime} \rightarrow E^{\prime}$, where $X^{\prime \prime}$ is the disjoint union of $X, X^{\prime}, f^{\prime \prime}\left|X=f, f^{\prime \prime}\right| X^{\prime}=f^{\prime}$, and $Y^{\prime \prime}$ is the disjoint union of $Y, Y^{\prime}, g^{\prime \prime}\left|Y=g, g^{\prime \prime}\right| Y^{\prime}=g^{\prime}$.

Note that for any other piecewise smooth triangulation $T^{\prime}$ for $M$, we can choose a third piecewise smooth triangulation $T^{\prime \prime}$ for $M$ that subdivides both $T$ and $T^{\prime}$. There are mappings $r: H_{c}^{q}\left(T^{\prime \prime}, L^{h}(p)\right) \rightarrow H_{c}^{q}\left(T ; L^{h}(p)\right)$ and $r^{\prime}: H_{c}^{q}\left(T^{\prime \prime} ; L^{h}(p)\right) \rightarrow H_{c}^{q}\left(T^{\prime} ; L^{h}(p)\right)$ obtained by amalgamating all the surgery blocks lying over the simplices of $T^{\prime \prime}$ that are contained in any given simplex 
of $T$ (or are contained in any given simplex of $T^{\prime}$ ). It can be deduced from the $\pi-\pi$ splitting theorem of C. T. C. Wall [64] that the maps $r, r^{\prime}$ are both isomorphism of abelian groups. Thus, we may define

$$
H_{c}^{q}\left(M ; L^{h}(p)\right) \equiv H_{c}^{q}\left(T ; L^{h}(p)\right)
$$

for any piecewise smooth triangulation $T$ for $M$. The previous remarks show that $H_{c}^{q}(M ; L(p))$ is independent of the particular $T$.

9.3. The cohomology groups $H_{c}^{q}(M ; \mathscr{L}(p))$. Let $T^{n}$ denote the $n$-dimensional torus, and let $p_{n}: E_{n} \rightarrow M$ denote the composite map

$$
E \times T^{n} \stackrel{\text { proj }}{\rightarrow} E \stackrel{p}{\rightarrow} M
$$

Let $T$ be a piecewise smooth triangulation for $M$ containing subcomplexes $K_{1}, K_{2}$ with $K_{2} \subset K_{1}$. Note that there is a map $H_{c}^{q}\left(K_{1}, K_{2} ; L^{h}\left(p_{n}\right)\right) \rightarrow$ $H_{c}^{q+1}\left(K_{1}, K_{2} ; L^{h}\left(p_{n+1}\right)\right)$ obtained by forming the Cartesian product of a normal map with the identity map id: $S^{1} \rightarrow S^{1}$. We define $H_{c}^{q}\left(K_{1}, K_{2} ; \mathscr{L}(p)\right)$ to be the direct limit of the sequence of group homomorphisms

$$
\begin{aligned}
H_{c}^{q+k}\left(K_{1}, K_{2} ; L^{h}\left(p_{k}\right)\right) & \rightarrow H_{c}^{q+k+1}\left(K_{1}, K_{2} ; L^{h}\left(p_{k+1}\right)\right) \\
& \rightarrow H_{c}^{q+k+2}\left(K_{1}, K_{2} ; L^{h}\left(p_{k+2}\right)\right) \rightarrow \cdots,
\end{aligned}
$$

where $k$ is a positive integer satisfying $q+k \geq 5$. Note that $H_{c}^{q}\left(K_{1}, K_{2} ; \mathscr{L}(p)\right)$ is defined for any integer $q$.

We define $H_{c}^{q}(M ; \mathscr{L}(p))$ to be equal to $H_{c}^{q}(T ; \mathscr{L}(p))$. Note that $H_{c}^{q}(M ; \mathscr{L}(p))$ is independent of the particular triangulation $T$.

9.4. The cohomology groups $H_{c}^{q}\left(M ; L^{-\infty}(p)\right)$. Note that in 9.3 we may choose $p_{n}^{\prime}: E_{n}^{\prime} \rightarrow M$ to be equal to the composite map

$$
E^{\prime} \times T^{n} \stackrel{\text { proj }}{\rightarrow} E^{\prime} \stackrel{p^{\prime}}{\rightarrow} M
$$

where $p^{\prime}: E^{\prime} \rightarrow M$ comes from 9.1. Let $T$ be a triangulation for $M$, and let $K_{1}, K_{2}$ be subcomplexes of $T$ with $K_{2} \subset K_{1}$. We note that there is a natural map $s_{n}: H_{c}^{q}\left(K_{1}, K_{2} ; L^{h}\left(p_{n}\right)\right) \rightarrow H_{c}^{q}\left(K_{1}, K_{2} ; \mathscr{L}(p)\right)$, called the splitting map, defined as follows. Let $f: X \rightarrow Y$ and $g: Y \rightarrow E_{n}^{\prime}$ represent $\alpha \in$ $H_{c}^{q}\left(K_{1}, K_{2} ; L^{h}\left(p_{n}\right)\right)$. For each $\Delta \in K_{1}$, put $g \mid Y(\Delta)$ in general position to the sequence $E^{\prime} \subset E_{1}^{\prime} \subset E_{2}^{\prime} \subset \cdots \subset E_{n}^{\prime}$. After doing 0- and 1-dimensional surgeries on each $g \mid Y(\Delta)$ and $f \mid X(\Delta)$, we may suppose that each of the maps $g: g^{-1}\left(E_{i}^{\prime}\right) \cap \partial_{2} Y(\Delta) \rightarrow E_{i}^{\prime}$ is two connected. We now consider the problem of splitting each of the homotopy equivalences $f: \partial_{2} X(\Delta) \rightarrow \partial_{2} Y(\Delta)$ along the sequence of submanifolds $\partial_{2} Y(\Delta) \cap g^{-1}\left(E^{\prime}\right) \subset \partial_{2} Y(\Delta) \cap g^{-1}\left(E_{1}^{\prime}\right) \subset \partial_{2} Y(\Delta) \cap$ $g^{-1}\left(E_{2}^{\prime}\right) \subset \cdots \subset \partial_{2} Y(\Delta) \cap g^{-1}\left(E_{n}^{\prime}\right)$. Note that if $\mathrm{Wh}(\pi \times A)=0$ holds for $\pi=\pi_{1}(F)$ and $A$ equals any finitely generated free abelian group, and if $\operatorname{dim}(F) \geq 5$, then the splitting theorem of [14] may be applied (inductively 
over the triangles of $K_{1}$ having increasing dimension and over the descending sequences $\left.\partial_{2} Y(\Delta) \cap g^{-1}\left(E_{n-1}^{\prime}\right) \supset \partial_{2} Y(\Delta) \cap g^{-1}\left(E_{n-2}\right) \supset \cdots \supset \partial_{2} Y(\Delta) \cap g^{-1}\left(E^{\prime}\right)\right)$ to get the desired splitting. If these conditions on $F$ are not met, we can still apply a stable version of the splitting theorem of [14] to conclude that for sufficiently large $l$ the homotopy equivalences $f_{l}: \partial_{2} X(\Delta) \times T^{l} \rightarrow \partial_{2} Y(\Delta) \times T^{l}$ can be split along the sequence of submanifolds $\left(\partial_{2} Y(\Delta) \cap g^{-1}\left(E^{\prime}\right)\right) \times T^{l} \subset$ $\left(\partial_{2} Y(\Delta) \cap g^{-1}\left(E_{1}^{\prime}\right)\right) \times T^{l} \subset\left(\partial_{2} Y(\Delta) \cap g^{-1}\left(E_{2}^{\prime}\right)\right) \times T^{l} \subset \cdots \subset\left(\partial_{2} Y(\Delta) \cap g^{-1}\left(E_{n}^{\prime}\right)\right) \times$ $T^{l}$, where $f_{l}=f \times$ id and id: $T^{l} \rightarrow T^{l}$ is the identity map on $T^{l}$. A relative version of the previous argument will assure that for each $\Delta \in K_{2}$ the homotopy equivalence $f: X(\Delta) \times T^{l} \rightarrow Y(\Delta) \times T^{l}$ is split along the sequence of manifolds $\left(Y(\Delta) \times g^{-1}\left(E^{\prime}\right)\right) \times T^{l} \subset\left(Y(\Delta) \times g^{-1}\left(E_{1}^{\prime}\right)\right) \times T^{l} \subset \cdots \subset(Y(\Delta) \times$ $\left.g^{-1}\left(E_{n}^{\prime}\right)\right) \times T^{l}$. Now put each of $f_{l}: X(\Delta) \times T^{l} \rightarrow Y(\Delta) \times T^{l}$ in general position to the sequence of manifolds $\left(Y(\Delta) \cap g^{-1}\left(E^{\prime}\right)\right) \times T^{l} \subset\left(Y(\Delta) \cap g^{-1}\left(E_{1}^{\prime}\right)\right) \times$ $T^{l} \subset\left(Y(\Delta) \cap g^{-1}\left(E_{2}^{\prime}\right)\right) \times T^{l} \subset \cdots \subset\left(Y(\Delta) \cap g^{-1}\left(E_{n}^{\prime}\right)\right) \times T^{l}$, and let the pair $f_{l}: f_{l}^{-1}\left(g^{-1}\left(E^{\prime}\right) \times T^{l}\right) \rightarrow g^{-1}\left(E^{\prime}\right) \times T^{l}, g_{l}: g^{-1}\left(E^{\prime}\right) \times T^{l} \rightarrow E^{\prime} \times T^{l}$ represent the image of $\alpha \in H_{c}^{q}\left(K_{1}, K_{2} ; L^{h}\left(p_{n}\right)\right)$ under the map $s_{n}: H_{c}^{q}\left(K_{1}, K_{2} ; L^{h}\left(p_{n}\right)\right) \rightarrow$ $H_{c}^{q}\left(K_{1}, K_{2} ; \mathscr{L}(p)\right)$, where $g_{l}=g \times$ id.

We define a splitting map

$$
S: H_{c}^{q}\left(K_{1}, K_{2}: \mathscr{L}(p)\right) \rightarrow H_{c}^{q}\left(K_{1}, K_{2} ; \mathscr{L}(p)\right)
$$

to be the direct limit of the splitting maps

$$
s_{n}: H_{c}^{q}\left(K_{1}, K_{2}: L^{h}\left(p_{n}\right)\right) \rightarrow H_{c}^{q}\left(K_{1}, K_{2} ; \mathscr{L}(p)\right) .
$$

Finally, we define $H_{c}^{q}\left(K_{1}, K_{2} ; L^{-\infty}(p)\right)$ to be the direct limit of the sequence

$$
H_{c}^{q}\left(K_{1}, K_{2} ; \mathscr{L}(p)\right) \stackrel{s}{\rightarrow} H_{c}^{q}\left(K_{1}, K_{2} ; \mathscr{L}(p)\right) \stackrel{s}{\rightarrow} H_{c}^{q}\left(K_{1}, K_{2} ; \mathscr{L}(p)\right) \stackrel{s}{\rightarrow} \cdots
$$

We define $H_{c}^{q}\left(M ; L^{-\infty}(p)\right)$ to be equal to $H_{c}^{q}\left(T ; L^{-\infty}(p)\right)$. Note that $H_{c}^{q}\left(M ; L^{-\infty}(p)\right)$ is independent of the particular triangulation $T$.

Remark 9.4(a). Note that if $p: E \rightarrow M$ is the trivial bundle, then the group $H_{c}^{q}\left(K_{1}, K_{2} ; L^{-\infty}(p)\right)$ is isomorphic to the group

$$
\left[\left(\left|K_{1}\right|,\left|K_{2}\right|, \mathbf{L}_{q}^{-\infty}\left(\pi_{1}(F), w^{\prime}\right)\right)\right]_{c}
$$

of homotopy classes of compactly supported mappings from the pair of spaces $\left(\left|K_{1}\right|,\left|K_{2}\right|\right)$, which underlie the subcomplexes $\left(K_{1}, K_{2}\right)$, into the surgery classifying space. This statement holds when $L^{-\infty}(p)$ is replaced by $\mathscr{L}(p)$ (or by $\left.L^{h}(p)\right)$ and $\mathbf{L}_{q}^{-\infty}\left(\pi_{1}(F), w^{\prime}\right)$ is replaced by the stable surgery classifying space $\mathscr{L}_{q}\left(\pi_{1}(F), w^{\prime}\right)$ (or by $\mathbf{L}_{q}^{h}\left(\pi_{1}(F), w^{\prime}\right)$ ). For further details regarding constructions as of 9.2-9.4, the reader is referred to $[58,57,39,40,6]$.

Remark 9.4(b). In the definitions of $H_{c}^{q}\left(K_{1}, K_{2} ; L^{h}(p)\right), H_{c}^{q}\left(K_{1}, K_{2} ; \mathscr{L}(p)\right)$, and $H_{c}^{q}\left(K_{1}, K_{2} ; L^{-\infty}(p)\right)$, we may assume that the 4-tuples $\left(Y(\Delta), \partial_{1} Y(\Delta)\right.$, $\left.\partial_{2} Y(\Delta), \wedge Y(\Delta)\right)$ of $9.2(\mathrm{~b})$ are finite Poincaré duality 4-tuples rather than 4- 
tuples of compact manifolds. There is then a natural inclusion of the cohomology groups defined in 9.2-9.4 (with $\left(Y(\Delta), \partial_{1} Y(\Delta), \partial_{2} Y(\Delta), Y(\Delta)\right.$ ) a 4-tuple of compact manifolds) into the corresponding cohomology groups of this remark. All three of these natural inclusions are isomorphisms. (Note that in the definition of $H_{c}^{q}\left(K_{1}, K_{2} ; L^{-\infty}(p)\right)$ given in 9.4, we must now use the transversality theorems from [38, 45] to put $g: Y(\Delta) \rightarrow E_{n}^{\prime}$ into general position to the sequence $E^{\prime} \subset E_{1}^{\prime} \subset \cdots \subset E_{n}^{\prime}$. Without loss of generality, we may assume that $\partial_{2} Y(\Delta) \neq \varnothing$ for all $\Delta \in K_{1}$, so that [38] may be applied.)

Remark 9.4(c). In the definitions of $H_{c}^{q}\left(M ; L^{h}(p)\right), H_{c}^{q}(M ; \mathscr{L}(p))$, and $H_{c}^{q}\left(M ; L^{-\infty}(p)\right)$ given in 9.2-9.4, and Remark 9.4(b), we may assume that $p: E \rightarrow M$ is only a homotopy fibration instead of a fiber bundle having a compact fiber.

We can now state the main result of this section. Define the amalgamation map $A^{q}: H_{c}^{q}\left(M ; L^{-\infty}(p)\right) \rightarrow L_{q+m}^{-\infty}\left(\pi_{1}(E), w\right), m=\operatorname{dim}(M)$, as follows. Let $\alpha \in H_{c}^{q}\left(M ; L^{-\infty}(p)\right)$ be represented by the pair of maps $f: X \rightarrow Y$, $g: Y \rightarrow E_{l}^{\prime}$ for $l$ large. Note that $f:(X, \partial X) \rightarrow(Y, \partial Y)$ is a normal map with compact support between manifold pairs (just amalgamate the orientation data and framing data given in $9.2(\mathrm{a})-(\mathrm{d}))$. Let $A^{q}(\alpha)$ be the obstruction to completing surgery on this normal map with respect to the fundamental group data given by $g: Y \rightarrow E_{l}^{\prime}$.

Theorem 9.5. For each integer $q$ and $m=\operatorname{dim}(M)$, the amalgamation map $A^{q}: H_{c}^{q}\left(M ; L^{-\infty}(p)\right) \rightarrow L_{q+m}^{-\infty}\left(\pi_{1}(E), w\right)$ is an isomorphism.

Let $K_{F / \text { Top }}^{*}()$ denote the cohomology defined in [39], and let $K_{F / \text { Top }, c}^{*}()$ denote the associated cohomology theory with compact supports. For any abelian group $A$, we let $H_{c}^{*}(, A)$ denote the ordinary cohomology theory with compact support and coefficients in $A$, and we let $K 0_{c}^{*}(, A)$ denote the real $K$-theory cohomology groups with compact support and coefficients in $A$. Let $Z_{(2)}$ denote the integers localized at 2 , and let $Z\left(\frac{1}{2}\right)$ denote the integers with 2 made invertible.

Corollary 9.5(a). Suppose that the bundle $p: E \rightarrow M$ is the trivial bundle. Then for each integer $q$, there is an isomorphism

$$
\bigoplus_{i=0}^{3} K_{F / \mathrm{TOP}, c}^{i}\left(M ; L_{q+i}^{-\infty}\left(\pi_{1}(F), w^{\prime}\right)\right) \cong L_{q+m}^{-\infty}\left(\pi_{1}(E), w\right)
$$

Corollary 9.5(b). Suppose that the bundle $p: E \rightarrow M$ is the trivial bundle. Then for each integer $q$, there are isomorphisms

$$
\bigoplus_{i=0}^{\infty} H_{c}^{i}\left(M ; L_{i+q}^{-\infty}\left(\pi_{1}(F), w^{\prime}\right) \otimes Z_{(2)}\right) \cong L_{q+m}^{-\infty}\left(\pi_{1}(E), w\right) \otimes Z_{(2)},
$$


and

$$
\bigoplus_{i=0}^{3} K 0_{c}^{i}\left(M ; L_{i+q}^{-\infty}\left(\pi_{1}(F), w^{\prime}\right) \otimes Z\left(\frac{1}{2}\right)\right) \cong L_{q+m}^{-\infty}\left(\pi_{1}(E), w\right) \otimes Z\left(\frac{1}{2}\right) .
$$

Proof of Corollaries 9.5(a) and (b). In [39], it was shown that there is a natural equivalence between the functors

$$
\left[, \mathbf{L}_{q}^{h}\left(\pi_{1}(F), w^{\prime}\right)\right]_{c} \text { and } \bigoplus_{i=0}^{3} K_{\overline{F / \mathrm{TOP}}, c}^{i}\left(, L_{q+i}^{h}\left(\pi_{1}(F), w^{\prime}\right)\right),
$$

where $\mathbf{L}_{q}^{h}\left(\pi_{1}(F), w^{\prime}\right)$ is the surgery classifying space having the surgery groups $L_{q+*}^{h}\left(\pi_{1}(F), w^{\prime}\right)$ for its homotopy groups. The arguments given in [39] may also be applied to conclude that there is a natural equivalence between the functors

$$
\left[, \mathbf{L}_{q}^{-\infty}\left(\pi_{1}(F), w^{\prime}\right)\right]_{c} \text { and } \bigoplus_{i=0}^{3} K \frac{i}{F / \mathrm{TOP}, c}\left(, L_{q+i}^{-\infty}\left(\pi_{1}(F), w^{\prime}\right)\right) \text {, }
$$

where $\mathbf{L}_{q}^{-\infty}\left(\pi_{1}(F), w^{\prime}\right)$ is the surgery classifying space having the surgery groups $L_{q+*}^{-\infty}\left(\pi_{1}(F), w^{\prime}\right)$ for its homotopy groups.

Now we can prove Corollary 9.5 (a) by noting that when $p: E \rightarrow M$ is the trivial bundle, then $H_{c}^{q}\left(M ; L^{-\infty}(p)\right)$ is isomorphic to $\left[M, \mathbf{L}_{q}^{-\infty}\left(\pi_{1}(F), w^{\prime}\right)\right]_{c}$, and then applying the results of the last paragraph and the conclusion of Theorem 9.5 .

We can derive Corollary 9.5 (b) from Corollary 9.5 (a) by recalling that the $\Omega$ spectrum $\overline{F / \text { TOP }}$ is four-fold periodic and that its zeroth level space $\overline{F / \text { TOP }_{0}}$ has the same $Z_{(2)}$-homotopy type as does the product $\prod_{j \geq 0} K(Z ; 4 j)$ of Eilenberg-MacLane spaces and has the same $Z\left(\frac{1}{2}\right)$-homotopy type as does B0.

This completes the proof of Corollaries 9.5(a) and (b).

Proof of Theorem 9.5. The functor $H_{c}^{*}\left(, ; L^{-\infty}(p)\right)$ satisfies many of the axioms of a cohomology theory. For example, if $K_{1}, K_{2}, K_{3}$ are subcomplexes of the triangulation $T$ for $M$ such that $K_{3} \subset K_{2} \subset K_{1}$, then there is a long exact sequence relating the groups $H_{c}^{*}\left(K_{1}, K_{2} ; L^{-\infty}(p)\right), H_{c}^{*}\left(K_{1}, K_{3} ; L^{-\infty}(p)\right)$, and $H_{c}^{*}\left(K_{2}, K_{3} ; L^{-\infty}(p)\right)$. There is also an excision isomorphism

$$
H_{c}^{q}\left(K_{1}, K_{2} ; L^{-\infty}(p)\right) \cong H_{c}^{q}\left(K_{1}-K_{3}^{0}, K_{2}-K_{3}^{0} ; L^{-\infty}(p)\right),
$$

where the $K_{3}^{0}$ denote the simplices of $K_{3}$ that intersect with $\operatorname{Int}\left(\left|K_{3}\right|\right)$. These two cohomology axioms are enough to guarantee the existence of a spectral sequence $E_{*, *}^{i}, i=1,2, \ldots$, satisfying the following properties (cf. [58]).

9.6. (a)

$$
E_{*, *}^{2}=H_{c}^{*}\left(\left|K_{1}\right|,\left|K_{2}\right| ; L_{q+*}^{-\infty}\left(\pi_{1}(F), w^{\prime}\right)_{t}\right) \text {. }
$$

(Here $H_{c}^{*}\left(\left|K_{1}\right|,\left|K_{2}\right| ; L_{q+j}^{-\infty}\left(\pi_{1}(F), w^{\prime}\right)_{t}\right)$ denotes the ordinary cohomology groups with compact support and having twisted coefficients in the surgery group 
$L_{q+j}^{-\infty}\left(\pi_{1}(F), w^{\prime}\right)$. The twisting of the coefficients is induced by the transition functions for the bundle $p: E \rightarrow M$.)

(b) $E_{*, *}^{i}$ converges to a filtration for $H_{c}^{q}\left(K_{1}, K_{2} ; L^{-\infty}(p)\right)$.

The remainder of the proof is carried out in the following three steps.

From this point on, we make the simplifying assumption that the boundary of the fiber manifold $F$ is empty.

Step I. For any piecewise smooth triangulation $T$ for $M$, we define a set $\eta\left(T ; L^{h}(p)\right)$ as follows. Any element $\alpha \in \eta\left(T ; L^{h}(p)\right)$ is represented by a map $h: N \rightarrow E \times I$ satisfying 9.7(a)-(c). Two such maps $h: N \rightarrow E \times I$, $h^{\prime}: N^{\prime} \rightarrow E \times I$ represent the same element in $\eta\left(T ; L^{h}(p)\right)$ if there is a third map $\bar{h}: \bar{N} \rightarrow(E \times I) \times I$ that satisfies $9.7(\mathrm{~d})-(\mathrm{f})$.

9.7. (a) $h:(N, \partial N) \rightarrow(E \times I, E \times \partial I)$ is a degree one normal map between manifold pairs that has compact support. $\partial N$ has two components $\partial_{+} N$, $\partial_{-} N$.

(b) $h: \partial_{-} N \rightarrow E \times \partial_{-} I$ is a homeomorphism.

(c) For each simplex $\Delta \in T$, the map $h:(N, \partial N) \rightarrow(E \times I, E \times \partial I)$ is in transverse position to the submanifold pair $p^{-1}(\Delta) \times(I, \partial I)$. Moreover, the restricted map $h: h^{-1}\left(p^{-1}(\Delta) \times I\right) \cap \partial_{+} N \rightarrow p^{-1}(\Delta) \times \partial_{+} I$ is a homotopy equivalence.

(d) $\bar{N}$ is a cobordism from $N$ to $N^{\prime}$. Set $\bar{N}_{\partial}=\operatorname{closure}\left(\partial \bar{N}-N-N^{\prime}\right)$. Then $\bar{h}:\left(\bar{N}, \bar{N}_{\partial}\right) \rightarrow(E \times I, E \times \partial I) \times I$ is a normal cobordism from $h$ to $h^{\prime}$ that has compact support.

(e) Let $\bar{N}_{\partial+}, \bar{N}_{\partial-}$ denote the two components of $\bar{N}_{\partial}$ that map to $\left(E \times \partial_{+} I\right) \times I,\left(E \times \partial_{-} I\right) \times I$. Then the restricted map $\bar{h}: \bar{N}_{\partial-} \rightarrow\left(E \times \partial_{-} I\right) \times I$ is a homeomorphism.

(f) For each simplex $\Delta \in T$, we have that the map $\bar{h}:\left(\bar{N}, \bar{N}_{\partial}, N \cup N^{\prime}\right) \rightarrow$ $(E \times I \times I, E \times \partial I \times I, E \times I \times \partial I)$ is in transverse position to

$$
\left(p^{-1}(\Delta) \times I \times I, p^{-1}(\Delta) \times \partial I \times I, p^{-1}(\Delta) \times I \times \partial I\right) .
$$

Moreover, the restricted map

$$
\bar{h}: \bar{h}^{-1}\left(p^{-1}(\Delta) \times I \times I\right) \cap N_{\partial_{+}} \rightarrow p^{-1}(\Delta) \times \partial_{+} I \times I
$$

is a homotopy equivalence.

By using the topological direct limit constructions of 9.3 and 9.4 , we can construct from $\eta\left(T ; L^{h}(p)\right)$ the sets $\eta(T ; \mathscr{L}(p))$ and $\eta\left(T ; L^{-\infty}(p)\right)$. Note that there is a natural (inclusion induced) map

$$
I: \eta\left(T ; L^{-\infty}(p)\right) \rightarrow H_{c}^{l+1}\left(T ; L^{-\infty}(p)\right),
$$

where $l=\operatorname{dim}(F)$. An Eilenberg-Whitney (surgery) obstruction argument shows that $I$ satisfies the following (see [40] for more details).

9.8. $I: \eta\left(T ; L^{-\infty}(p)\right) \rightarrow H_{c}^{l+1}\left(T ; L^{-\infty}(p)\right)$ is a set isomorphism.

The purpose of this step is to verify the following claim. 
9.9. To complete the proof of Theorem 9.5, it will suffice to show that the composite map

$$
\eta\left(T ; L^{-\infty}(p)\right) \stackrel{I}{\rightarrow} H_{c}^{l+1}\left(T ; L^{-\infty}(p)\right) \stackrel{A^{1}}{\rightarrow} L_{l+1+m}^{-\infty}\left(\pi_{1}(E) ; w\right)
$$

is a set isomorphism for every fiber bundle $p: E \rightarrow M$ having a compact closed $l$-dimensional manifold for fiber and for any $l$.

Note that it follows from 9.8 that 9.9 is true for $q=l+1$ in the statement of Theorem 9.5.

It remains to be seen how to reduce the general version of Theorem 9.5 to the special case $q=l+1$. Consider the following commutative diagram

$$
\begin{array}{ccc}
H_{c}^{q+4}\left(T ; L^{-\infty}(p)\right) & \stackrel{A^{q+4}}{\rightarrow} & L_{q+4+m}^{-\infty}\left(\pi_{1}(E), w\right) \\
P_{1} \uparrow & & \uparrow P_{2} \\
H_{c}^{q}\left(T ; L^{-\infty}(p)\right) & \stackrel{A^{q}}{\rightarrow} & L_{q+m}^{-\infty}\left(\pi_{1}(E), w\right)
\end{array}
$$

where the maps $P_{1}, P_{2}$ are obtained by forming the Cartesian product with the identity map id: $C P^{2} \rightarrow C P^{2}$ on (real) 4-dimensional complex projective space. Wall's surgery periodicity theorem states that $P_{2}$ is an isomorphism (see [64]). On the other hand, forming the product with id: $C P^{2} \rightarrow C P^{2}$ induces a map between spectral sequences, given in 9.6 for $H_{c}^{q+4}\left(T ; L^{-\infty}(p)\right)$ and $H_{c}^{q}\left(T ; L^{-\infty}(p)\right)$, which (by Wall's surgery periodicity) is an isomorphism on the $E_{* * *}^{2}$-terms. Thus, $P_{1}$ is also an isomorphism.

Note that it follows from the results of the preceding paragraph that Theorem 9.5 is true for $q$ if and only if it is true for $q+4$. Choose integers $r, k$ so that $r \geq 2, r+l+1=4 k+q$. Consider the fiber bundle projection $\bar{p}: \bar{E} \rightarrow M$ that is equal to the composite map

$$
E \times S \stackrel{\text { proj }}{\rightarrow} E \stackrel{p}{\rightarrow} M
$$

where $S^{r}$ is the $r$-dimensional sphere. Note that there is the commutative diagram

$$
\begin{array}{ccc}
H_{c}^{4 k+q}\left(T ; L^{-\infty}(p)\right) & \stackrel{A^{4 k+4}}{\rightarrow} & L_{4 k+q+m}^{-\infty}\left(\pi_{1}(E), w\right) \\
J_{1} \uparrow & & \uparrow J_{2} \\
H_{c}^{4 k+q}\left(T ; L^{-\infty}(\bar{p})\right) & \stackrel{\bar{A}^{4 k+4}}{\rightarrow} & L_{4 k+q+m}^{-\infty}\left(\pi_{1}(\bar{E}), \bar{w}\right) \\
& & \\
\eta\left(T ; L^{-\infty}(\bar{p})\right) & &
\end{array}
$$

where $J_{1}, J_{2}$ are induced by the projection map $\bar{E}=E \times S^{r} \rightarrow E$. The maps $\bar{I}, J_{1}, J_{2}$ are all set isomorphisms. Thus Theorem 9.5 is true for $q+4 k$ if 9.9 holds for $\bar{p}$ and $\bar{l}=r+l$.

This completes Step I.

Step II. In this step, we complete the proof of Theorem 9.5 for $M$ that satisfy the following hypothesis. 
Hypothesis 9.10. $M$ is the topological product $M^{\prime} \times R$ equipped with the metric of (3.8.4), where $M^{\prime}$ is another complete real hyperbolic manifold. Moreover, $M$ has even dimension.

By 9.9, it will suffice to show that

$$
A^{l+1} \circ I: \eta\left(T ; L^{-\infty}(p)\right) \rightarrow L_{l+1+m}^{-\infty}\left(\pi_{1}(E), w\right)
$$

is a set isomorphism. We shall accomplish this by applying Corollary 4.3, Remark 4.3, Theorem 5.5, and Remark 5.6. Before carrying out the details, we need some more notation.

We let $\xi, C(\xi),{ }_{\varepsilon} \tau^{\prime}$ be as in 5.1. Let $\bar{p}: \bar{E} \rightarrow M$ denote the fiber bundle projection that is the composite map

$$
\operatorname{closure}\left(C(\xi)-{ }_{\varepsilon} \tau^{\prime}\right) \stackrel{\text { proj }}{\rightarrow} E \stackrel{p}{\rightarrow} M .
$$

Note that the transfer construction given in 4.2 can be suitably modified to give a commutative diagram

$$
\begin{array}{ccc}
H_{c}^{q+4 k}\left(T ; L^{-\infty}(\bar{p})\right) & \stackrel{\bar{A}^{q+4 k}}{\rightarrow} & L_{q+4 k+m}^{-\infty}\left(\pi_{1}(\bar{E}), \bar{w}\right) \\
\sigma_{1} \uparrow & & \uparrow \sigma_{2} \\
H_{c}^{q}\left(T ; L^{-\infty}(p)\right) & \stackrel{A^{q}}{\rightarrow} & L_{q+m}^{-\infty}\left(\pi_{1}(E), w\right)
\end{array}
$$

where $\sigma_{1}, \sigma_{2}$ are transfer maps and where $2+2 k=\operatorname{dim}(M)$. It follows from Corollary 4.3 and Remark 4.3 that $\sigma_{2}$ is an isomorphism. On the other hand, the transfer construction discussed in 4.2 gives rise to a mapping between spectral sequences - which are associated to $H_{c}^{q}\left(T ; L^{-\infty}(p)\right)$ and $H_{c}^{q+4 k}\left(T ; L^{-\infty}(\bar{p})\right)$ as in 9.6 -which (by 4.3) is an isomorphism on the $E_{*, *}^{2}$-terms. Thus, $\sigma_{1}$ is also an isomorphism. Combining the results of this paragraph with 9.8 , we get the following.

9.11. (a) There is a commutative diagram

$$
\begin{array}{ccc}
\eta\left(T ; L^{-\infty}(\bar{p})\right) & \bar{A}^{I+1+4 k} \circ \bar{I} & L_{l+1+4 k+m}^{-\infty}\left(\pi_{1}(\bar{E}), \bar{w}\right) \\
\bar{I}^{-\circ} \circ \sigma_{1} \circ I \uparrow & & \uparrow \sigma_{2} \\
\eta\left(T ; L^{-\infty}(p)\right) & \stackrel{A^{l+1} \circ I}{\rightarrow} & L_{l+1+m}^{-\infty}\left(\pi_{1}(E), w\right)
\end{array}
$$

(b) The transfers $\bar{I}^{-1} \circ \sigma_{1} \circ I$ and $\sigma_{2}$ are isomorphisms.

Now we show that $A^{l+1} \circ I: \eta\left(T ; L^{-\infty}(p)\right) \rightarrow L_{l+1+m}^{-\infty}\left(\pi_{1}(E), w\right)$ is surjective. Choose any $\alpha \in L_{l+1+m}^{-\infty}\left(\pi_{1}(E), w\right)$, and represent $\alpha$ by a degree one normal map $h:\left(N, \partial_{ \pm} N\right) \rightarrow\left(E \times I, E \times \partial_{ \pm} I\right)$ that has compact support and is such that $h: \partial_{-} N \rightarrow E \times \partial_{-} I$ is a homeomorphism and $h: \partial_{+} N \rightarrow E \times \partial_{+} I$ is a homotopy equivalence. By applying Theorem 5.5 and Remark 5.6 to the transfer of $h: \partial_{+} N \rightarrow E \times \partial_{+} I$, we may conclude

9.12. For any $\alpha \in L_{l+1+m}^{-\infty}\left(\pi_{1}(E), w\right)$, we have $\sigma_{2}(\alpha) \in \operatorname{Image}\left(\bar{A}^{l+1+4 k} \circ \bar{I}\right)$. Now 9.11 and 9.12 together show that $A^{l+1} \circ I$ is surjective. 
Finally, we show that $A^{l+1} \circ I: \eta\left(T ; L^{-\infty}(p)\right) \rightarrow L_{l+1+m}^{-\infty}\left(\pi_{1}(E), w\right)$ is injective. Choose any $\alpha, \alpha^{\prime} \in \eta\left(T ; L^{-\infty}(p)\right)$ such that $A^{l+1} \circ I(\alpha)=A^{l+1} \circ I\left(\alpha^{\prime}\right)$. We may choose mappings $h: N \rightarrow E \times I, h^{\prime}: N^{\prime} \rightarrow E \times I$ that represent $\alpha$, $\alpha^{\prime}$ as in 9.7(a)-(c). Since $A^{l+1} \circ I(\alpha)=A^{l+1} \circ I\left(\alpha^{\prime}\right)$, we may suppose that the $h, h^{\prime}$ are related to a third map $\bar{h}: \bar{N} \rightarrow E \times I \times I$ as follows.

9.13 .

(a) $9.7(\mathrm{~d})$ and (e) are satisfied by $\bar{h}, h, h^{\prime}$.

(b) The restricted map $\bar{h}: \bar{N}_{\partial_{+}} \rightarrow\left(E \times \partial_{+} I\right) \times I$ is a homotopy equivalence.

By applying Theorem 5.5 and Remark 5.6 to the transfer of the map $\bar{h}: N_{\partial_{+}}$ $\rightarrow\left(E \times \partial_{+} I\right) \times I\left(\bmod \bar{h} \mid\left(N_{\partial_{+}} \cap\left(N \cup N^{\prime}\right)\right)\right.$, we may conclude

9.14. For any $\alpha, \alpha^{\prime} \in \eta\left(T ; L^{-\infty}(p)\right)$ that satisfy $A^{l+1} \circ I(\alpha)=A^{l+1} \circ I\left(\alpha^{\prime}\right)$, we have that $\bar{I}^{-1} \circ \sigma_{1} \circ I(\alpha)=\bar{I}^{-1} \circ \sigma_{1} \circ I\left(\alpha^{\prime}\right)$.

Now 9.11 and 9.14 together show that $A^{l+1} \circ I$ is injective.

This completes Step II.

Step III. In this step, we complete the proof of Theorem 9.5 without Hypothesis 9.10 .

Choose an integer $i>0$ such that $\operatorname{dim}(M)+i$ is an even integer. Let $M \times R^{i}$ be equipped with the hyperbolic enlargement Riemannian structure of $[25,1.6]$. Let $\bar{p}: \bar{E} \rightarrow M \times R^{i}$ denote the bundle projection that is the product map

$$
E \times R^{i} \stackrel{p \times 1}{\rightarrow} M \times R^{i}
$$

Note that $M \times R^{i}$ satisfies Hypothesis 9.10. Thus, for each integer $q$ the amalgamation map $\bar{A}^{q}: H_{c}^{q}\left(M \times R^{i} ; L^{-\infty}(\bar{p})\right) \rightarrow L_{q+m+i}^{-\infty}\left(\pi_{1}(\bar{E}), \bar{w}\right)$ is an isomorphism. On the other hand, there is a commutative diagram

$$
\begin{array}{ccc}
H_{c}^{q+i}\left(M ; L^{-\infty}(p)\right) & \stackrel{A^{q+i}}{\rightarrow} & L_{q+m+i}^{-\infty}\left(\pi_{1}(E), w\right) \\
S_{1} \uparrow & & \uparrow S_{2} \\
H_{c}^{q}\left(M \times R^{i} ; L^{-\infty}(\bar{p})\right) & \stackrel{\bar{A}^{q}}{\rightarrow} & L_{q+m+i}^{-\infty}\left(\pi_{1}(\bar{E}), \bar{w}\right)
\end{array}
$$

such that both $S_{1}, S_{2}$ are isomorphisms. The map $S_{2}$ is induced by the projection $\bar{E} \rightarrow E$. To get $S_{1}$, we choose triangulations $T, \bar{T}$ for $M, M \times R^{i}$ such that for each simplex $\Delta \in T$, the preimage of $\Delta$ in $M \times R^{i}$ (under the projection map $M \times R^{i} \rightarrow M$ ) is a subcomplex of $\bar{T}$, which we denote by $\bar{\Delta}$. Now we use the equivalences

$$
\begin{gathered}
H_{c}^{q+i}\left(M ; L^{-\infty}(p)\right) \simeq H_{c}^{q+i}\left(T, L^{-\infty}(p)\right), \\
H_{c}^{q}\left(M \times R^{i} ; L^{-\infty}(\bar{p})\right) \cong H_{c}^{q}\left(\bar{T} ; L^{-\infty}(p)\right),
\end{gathered}
$$

and define

$$
S_{1}: H_{c}^{q}\left(\bar{T} ; L^{-\infty}(\bar{p})\right) \rightarrow H_{c}^{q+i}\left(T ; L^{-\infty}(p)\right)
$$

by amalgamation over each subcomplex $\bar{\Delta} \subset \bar{T}$ with $\Delta \in T$. (Note that any $\alpha \in H_{c}^{q}\left(\bar{T} ; L^{-\infty}(\bar{p})\right)$ can be represented by a collection $h_{e}: X_{e} \rightarrow Y_{e}$, $e \in \bar{T}$, of degree one normal maps on compact manifolds such that $\left(X_{e}, X_{e}\right)$ are 
nonempty for only finitely many simplices $e \in \bar{T}$. Thus, when we amalgamate the $h_{e}, e \in \bar{T}$, over each $\bar{\Delta}$, we get normal maps $h_{\Delta}: X_{\Delta} \rightarrow Y_{\Delta}$ on compact manifolds for each $\Delta \in T$, as is required in 9.2(b).)

This completes the proof of Theorem 9.5.

\section{Applications}

In this section, we discuss various consequences of Theorem 9.5, the main result of this paper. Our applications shed light on the following three general problems.

Problem 1. Decide when two homotopically equivalent manifolds are homeomorphic.

Problem 2. Give useful criteria allowing the replacement of a continuous map by a homotopic block bundle projection.

Problem 3. "Calculate" the homotopy type of the homeomorphism or diffeomorphism group of a manifold.

Our results on these problems make important use of the earlier works of many people. What we do shows how older, general theories can be effectively used in light of the main result of this paper and its two predecessors, [23, 25], to "solve" these problems in certain interesting instances.

Throughout this section, $M$ will denote a complete (connected) real hyperbolic manifold, and $m$ will denote the dimension of $M$. We will usually place restrictions on $m$. Generally, $m$ differs from 3 and 4 and, when discussing Problem 3, $m>10$.

Recall that a homotopy-topological structure on $M \times R^{n}$ (where $n \geq 0$ ) is a pair $(N, f)$, where $N$ is a (topological) manifold and $f: N \rightarrow M \times R^{n}$ is a proper homotopy equivalence that is a homeomorphism over the complement of some compact set in $M \times R^{n}$. (Note that $M \times R^{0}=M$.) Two structures $\left(N_{1}, f_{1}\right)$ and $\left(N_{2}, f_{2}\right)$ are equivalent if there exists a homeomorphism $g: N_{1} \rightarrow$ $N_{2}$ such that $f_{2} \circ g$ and $f_{1}$ are properly homotopic via a homotopy that is constant over the complement of some compact set in $N_{1}$. Let $\mathscr{S}\left(M \times R^{n}\right)$ denote the set of equivalence classes of homotopy-topological structures on $M \times$ $R^{n}$. (See [64, 43, 62, and 46] for more details.) Our main result, Theorem 9.5, together with Corollary A.18.3 (see the appendix) combined with surgery theory $[64,43]$ has the following immediate consequence.

Theorem 10.1. If $n$ is any nonnegative integer larger than $4-m$, then the structure set $\mathscr{S}\left(M \times R^{n}\right)$ contains only one element, and this element is represented by the identity map of $M \times R^{n}$.

Recall that in the case of a compact manifold $W$ with possibly nonempty boundary $\partial W$ (where $W h \pi_{1} W=0$ ), a homotopy-topological structure rel $\partial$ on $W$ is a homotopy equivalence $f_{1}: W_{1} \rightarrow W$, where $W_{1}$ is another manifold with boundary such that $f_{1}$ maps $\partial W_{1}$ homeomorphically to $\partial W$. Two 
structures $\left(W_{1}, f_{1}\right)$ and $\left(W_{2}, f_{2}\right)$ are equivalent if there exists a homeomorphism $g: W_{2} \rightarrow W_{1}$ with $f_{1} \circ g$ homotopic to $f_{2}$ rel $\partial$. Let $\mathscr{S}(W$ rel $\partial)$ denote the set of equivalence classes of these structures. When $M$ has finite volume, it has a manifold compactification $\bar{M}$ due to Gromov and Margulis [32]. Let $W=\bar{M} \times D^{n}$, where $D^{n}$ denotes the closed ball in $R^{n}$ of unit radius and center 0 . (Recall that $\mathrm{Wh} \pi_{1} W=0$ by [23, Theorem 1].) One easily sees that there is a one-to-one correspondence between $\mathscr{S}(W$ rel $\partial)$ and $\mathscr{S}($ Int $W)=\mathscr{S}\left(M \times R^{n}\right)$ under which the elements represented by the identity maps correspond. Hence, Theorem 10.1 has the following consequence.

Corollary 10.2. Suppose $M$ has finite volume, and let $\bar{M}$ denote the GromovMargulis manifold compactification of $M$. Then the set $\mathscr{S}\left(\bar{M} \times D^{n}\right.$ rel $\left.\partial\right)$ has cardinality 1 provided $m+n>4$.

Specializing Corollary 10.2 by assuming $M$ is compact and $n=0$ yields the following result.

Corollary 10.3. Let $N$ be a compact (connected) topological manifold such that $N$ and $M$ have isomorphic fundamental groups and $N$ is aspherical (i.e., the universal covering space of $N$ is contractible). Then $N$ and $M$ are homeomorphic ${ }^{3}$ provided the dimension of $M$ differs from 3 and 4 .

Remarks 10.1. It is an immediate consequence of results of Knesser [44] and Milnor [48] that the truth of Corollary 10.3 when $m=3$ would imply the truth of the Poincaré Conjecture. Although our calculations of the surgery obstruction groups ( $L$-groups) and the Whitehead group are true even when $m=3$ or 4 , classical surgery theory does not work when $m=3$ and is unknown to work when $m=4$ for these fundamental groups. (Freedman and Quinn have shown that (topological) surgery theory does work for 4-dimensional manifolds with virtually poly- $Z$ fundamental groups [31].) Hence, we cannot prove Corollary 10.3 unless we assume that $m \neq 3$ and 4 .

Recall that an $n$-dimensional manifold $N$ has a (real) hyperbolic structure if and only if it can be covered by local coordinate charts with values in hyperbolic space $H^{n}$ such that change of coordinates is via isometries of open subsets of $H^{n}$. Corollary 10.3 together with Mostow's rigidity theorem [50] yields a characterization of hyperbolic structures on compact manifolds whose dimension is greater then 4 ; i.e., Corollary 10.3 is an existence theorem for hyperbolic structures while Mostow's work is the uniqueness theorem.

Corollary 10.4. A compact (connected) topological manifold $N$ of dimension $n \neq 3$ and 4 has a (real) hyperbolic structure if and only if

(i) $N$ is aspherical and

(ii) the fundamental group of $N$ is isomorphic to a discrete cocompact subgroup of the Lie group $O(n, 1)$.

\footnotetext{
${ }^{3}$ We construct examples, in [67], of nondiffeomorphic compact smooth manifolds $M$ and $N$ with isomorphic fundamental groups (and $\operatorname{dim} M>4$ ) where $M$ is a real hyperbolic manifold and $N$ supports a Riemannian metric whose sectional curvatures are all (strictly) negative.
} 
Furthermore, by Mostow's rigidity theorem, the structure is unique (up to isometry) provided $n>2$.

Remark 10.2. The Lie group $O(n, 1)$ consists of all $(n+1) \times(n+1)$ matricies $A$ with real number entries satisfying the equation

$$
A D(\text { transpose } A)=D \text {, }
$$

where $D$ is the diagonal $(n+1) \times(n+1)$ matrix such that $D_{i i}=1$ (if $1 \leq i \leq n$ ) and $D_{n+1, n+1}=-1$.

More generally, if $M$ has finite volume but perhaps is not compact, we have the following generalization of Corollary 10.3.

Corollary 10.5. Suppose that $M$ has finite volume and $m \neq 3,4$, and 5. Let $N$ be any topological manifold that is properly homotopically equivalent to $M$, then $N$ and $M$ are homeomorphic. In fact, any proper homotopy equivalence is properly homotopic to a homeomorphism.

Proof. Let $f: N \rightarrow M$ be a proper homotopy equivalence. Since each connected component $B$ of $\partial \bar{M}$ is a compact flat Riemannian manifold, both $K_{0}\left(\mathbf{Z}_{1} B\right)$ and $\mathrm{Wh}\left(\pi_{1} B\right)$ vanish by the main result of [16]. Hence, Siebenmann's thesis [61] can be used to construct a manifold compactification $\bar{N}$ for $N$ and a homotopy equivalence $\bar{f}:(\bar{N}, \partial \bar{N}) \rightarrow(\bar{M}, \partial \bar{M})$ such that

$$
\bar{f}: N=\bar{N}-\partial \bar{N} \rightarrow M=\bar{M}-\partial \bar{M}
$$

is properly homotopically equivalent to $f$. By the main result of [21], $\bar{f}: \partial \bar{N}$ $\rightarrow \partial \bar{M}$ is homotopic to a homeomorphism, so we may assume that $\bar{f}$ maps $\partial \bar{N}$ homeomorphically to $\partial \bar{M}$. An application of Corollary 10.2, with $n=0$, completes the proof.

Our penultimate application of Theorem 9.5 to Problem 1 is the following result, which was announced in [27, Theorem 1], where $N$ denotes a compact topological manifold with boundary $\partial N$ that is decomposed as the union of compact codimension-zero submanifolds $\partial_{1} N$ and $\partial_{2} N$ with

$$
\partial_{1} N \cap \partial_{2} N=\partial\left(\partial_{1} N\right)=\partial\left(\partial_{2} N\right) .
$$

Corollary 10.6. Let $f:\left(N, \partial_{1} N, \partial_{2} N\right) \rightarrow\left(\bar{M} \times D^{n}, \bar{M} \times \partial D^{n}, \partial \bar{M} \times D^{n}\right)$ be a homotopy equivalence of triads such that $f$ restricted to $\partial_{1} N$ is a homeomorphism and $n+m>5$. Then $f$ is homotopic to a homeomorphism via a homotopy of triad maps that is constant on $\partial_{1} N$.

Proof. Since $\partial \bar{M}$ is a compact flat Riemannian manifold, the main result of [21] allows us to homotope $f: \partial_{2} N \rightarrow \partial \bar{M} \times D^{n}$ to a homeomorphism via a homotopy that is constant on $\partial\left(\partial_{2} N\right)$. An application of Corollary 10.2 (after using the homotopy extension theorem) completes the proof.

Frank Quinn, in his Ph.D. thesis [57] and in [58], developed a general theory for studying Problem 2. The following result is an immediate consequence of Theorem 9.5 and his theory. (Recall that a group $\Gamma$ is $K$-flat if $\mathrm{Wh}(\Gamma \times C)$ 
vanishes, where $C$ is any finitely generated free abelian group; cf. [23, Theorem 1].)

Theorem 10.7. Let $p: E \rightarrow M$ be a continuous surjection, where $E$ is a compact manifold whose dimension is greater than $m+4$. Assume that the homotopy fiber $F$ of $p$ is a connected finite polyhedron and that both $\pi_{1} F$ and $\pi_{1} E$ are $K$-flat. Then $p$ is homotopic to a block bundle projection.

We now state our final result about Problem 1.

Corollary 10.8. Let $M_{1}, \ldots, M_{s}$ (where $s \geq 1$ ) be a finite sequence of compact (connected) real hyperbolic manifolds and $N$ a compact (connected) aspherical manifold such that $\pi_{1} N$ is a virtually poly- $\mathrm{Z}$ group. (We allow the cases where either $N$ or all of the $M_{i}$ are only a single point.) Then the set

$$
\mathscr{S}\left(M_{1} \times \cdots \times M_{s} \times N \times D^{n} \operatorname{rel} \partial\right)
$$

has cardinality 1 provided $n+\operatorname{dim} N+\sum_{i=1}^{s} \operatorname{dim} M_{i} \neq 3$ or 4 .

Remark 10.3. We showed [23, Corollary 2] that $\Gamma$ is $K$-flat, where $\Gamma$ is the fundamental group of $M_{1} \times \cdots \times M_{s} \times N$.

Proof of Corollary 10.8. Because of [13] and [43], $\mathscr{S}\left(M_{1} \times \cdots \times M_{s} \times N \times D^{n}\right.$ rel $\left.\partial\right)$ is in one-to-one correspondence with a subset of $\mathscr{S}\left(M_{1} \times \cdots \times M_{s} \times N \times T^{n+8}\right)$. Consequently, it suffices to show that this second set has cardinality 1 . We show this by induction on $s$. The inductive step is a consequence of two facts, which we proceed to demonstrate. Let

$$
\hat{p}: M_{1} \times \cdots \times M_{s} \times N \times T^{n+8} \rightarrow M_{s}
$$

denote the canonical projection onto the third to the last factor, and let $p=$ $\hat{p} \circ f$, where

$$
f: W \rightarrow M_{1} \times \cdots \times M_{s} \times N \times T^{n+8}
$$

represents an element of $\mathscr{S}\left(M_{1} \times \cdots \times M_{s} \times N \times T^{n+8}\right)$. Because of Theorem 10.7 and Remark 10.3 (recall that $N \times T^{n+8}$ is an aspherical manifold whose fundamental group is virtually poly- $Z$ and the homotopy fiber $F$ of $p$ is $M_{1} \times$ $\left.\cdots \times M_{s-1} \times N \times T^{n+8}\right), p$ is homotopic to a block bundle projection. This is the first fact.

For the same reason given in the first sentence of this proof, $\mathscr{S}\left(F \times D^{i}\right.$ rel $\left.\partial\right)$ is in one-to-one correspondence with a subset of $\mathscr{S}\left(F \times T^{i}\right)$, where $i$ is any nonnegative integer. Consequently, our inductive assumption implies that $\mathscr{S}\left(F \times D^{i}\right.$ rel $\left.\partial\right)$ has cardinality 1 provided $s>1$. When $s=1$, this is still true because of the main result of [26]. This is the second fact.

One can use these two facts to show easily that $f$ is homotopic to a homeomorphism. This completes the inductive step of our proof.

Now we use Theorem 9.5 to shed light on Problem 3. For any topological manifold $V$, Top $V$ denotes the space of all compactly supported selfhomeomorphisms of $V$, i.e., if $f \in \operatorname{Top} V$, then there exists a compact subset 
$K$ of $V$ such that the restriction of $f$ to $V-K$ is the inclusion map. If $V$ is an open subset of a manifold $N$, then the inclusion map $\sigma: V \rightarrow N$ induces a canonical continuous map

$$
\hat{\sigma}: \operatorname{Top} V \rightarrow \operatorname{Top} M
$$

that is determined by the formula

$$
\hat{\sigma}(f)(x)= \begin{cases}x, & \text { if } x \in N-V, \\ f(x), & \text { if } x \in V\end{cases}
$$

where $f \in \operatorname{Top} V$.

We next introduce some notation for important geometric objects related to $M$; e.g., $\mathscr{C}_{1}, \mathscr{C}_{2}, \ldots$ denote the "cusps" of $M$, given by Margulis' Lemma applied to $M_{\mu_{0}}$ (these are the components of $M_{\mu_{0}}$ whose closures are not compact, cf. Lemma 3.2), and

$$
\phi_{i}: \mathscr{C}_{i} \rightarrow M
$$

denote the inclusion maps. Let $g_{i}: S^{1} \rightarrow M$ be a sequence of smooth immersions such that their images $g_{1}\left(S^{1}\right), g_{2}\left(S^{1}\right), \ldots$ are an enumeration of the set of all $t$-simple closed geodesics in $M$, and let $g_{i}^{\prime}: S^{1} \rightarrow M$ be smooth embeddings such that their images are pairwise disjoint (we assume that $\operatorname{dim} M>2$ ) and each $g_{i}^{\prime}$ is regularly homotopic to $g_{i}$ via a short regular homotopy (say of length less than 1). Let $T_{i}$ be open tubular neighborhoods of the $g_{i}^{\prime}\left(S^{1}\right)$ such that the sets $T_{i}$ are pairwise disjoint, and let

$$
\psi_{i}: T_{i} \rightarrow M
$$

denote the inclusion maps.

If $N$ is a topological manifold, $G(N)$ denotes the $H$-space of self-homotopy equivalences of $N$ with compact support. Note that Top $N$ is a subspace of $G(N)$ and, if $\sigma: V \rightarrow N$ is the inclusion map of an open subset, then $\hat{\sigma}: \operatorname{Top} V \rightarrow \operatorname{Top} N$ extends canonically to a continuous map

$$
\hat{\sigma}: G(V) \rightarrow G(N) .
$$

Recall that the space $G(N) / \operatorname{Top} N$ is the homotopy fiber of the map $B \operatorname{Top} N$ $\rightarrow B G(N)$. Hence, $\hat{\sigma}$ induces a continuous map, also denoted by $\hat{\sigma}$,

$$
\hat{\sigma}: G(V) / \operatorname{Top} V \rightarrow G(N) / \operatorname{Top} N \text {. }
$$

If $f: X \rightarrow Y$ is a continuous map, then

$$
(f)_{s}: \pi_{s} X \rightarrow \pi_{s} Y
$$

denotes the induced homomorphism on the homotopy groups $\pi_{s}$. Also, if $G_{i}$ is a sequence of groups and $\eta_{i}: G_{i} \rightarrow G$ is a sequence of group homomorphisms satisfying

$$
\eta_{i}(x) \eta_{j}(y)=\eta_{j}(y) \eta_{i}(x)
$$


for all induces $i$ and $j$ and any pair of elements $x \in G_{i}$ and $y \in G_{j}$, then $\sum_{i} \eta_{i}: \bigoplus_{i} G_{i} \rightarrow G$ denotes the homomorphism defined by

$$
\left(\sum_{i} \eta_{i}\right)\left(x_{1}, x_{2}, \ldots\right)=\sum_{i}\left(\eta_{i}\left(x_{i}\right)\right)
$$

where $x_{i} \in G_{i}$ and $x_{i}=1$ for all but a finite number of indices $i$. If this sequence has length 2 , we use the notation $\eta_{1}+\eta_{2}$ for $\sum_{i} \eta_{i}$. Our primary result on Problems 3 is expressed in this notation as follows.

Theorem 10.9. The homomorphism $\sum_{i}\left(\hat{\phi}_{i}\right)_{s}+\sum_{i}\left(\hat{\psi}_{i}\right)_{s}$ is an isomorphism provided $0<s \leq(m-4) / 3$ and $m>10$. Note that the domain of $\sum_{i}\left(\hat{\phi}_{i}\right)_{s}+$ $\sum_{i}\left(\hat{\psi}_{i}\right)_{s}$ is

$$
\bigoplus_{i} \pi_{s}\left(G\left(\mathscr{C}_{i}\right) / \operatorname{Top} \mathscr{C}_{i}\right) \oplus \bigoplus_{i} \pi_{s}\left(G\left(T_{i}\right) / \operatorname{Top} T_{i}\right),
$$

and its range is $\pi_{s}(G(M) /$ Top $M)$.

Proof. Recall that, for any manifold $N$, we can interpolate between Top $N$ and $G(N)$ the simplicial group Top $N$ of block homeomorphisms of $N$ having compact support, cf. [33]. Because of Quinn's function space interpretation of the surgery exact sequence $[57,58]$, Theorem 10.1 implies that $G(M) / \widetilde{\operatorname{Top}} M$ is contractible. Hence, the natural map

$$
\widetilde{\operatorname{Top}} M / \operatorname{Top} M \rightarrow G(M) / \operatorname{Top} M
$$

is a homotopy equivalence. Recall that each cusp $\mathscr{C}_{i}$ and each tube $T_{j}$ is homeomorphic to the total space of a real vector bundle whose base space is a compact flat Riemannian manifold, a circle in the case of $T_{j}$. Thus, the same reasoning, but using the main result of [21] in place of Theorem 10.1, yields that the natural maps

$$
\begin{aligned}
& \widetilde{\operatorname{Top}} \mathscr{C}_{i} / \operatorname{Top} \mathscr{C}_{i} \rightarrow G\left(C_{i}\right) / \operatorname{Top} \mathscr{C}_{i}, \\
& \widetilde{\operatorname{Top}} T_{j} / \operatorname{Top} T_{j} \rightarrow G\left(T_{j}\right) / \operatorname{Top} T_{j}
\end{aligned}
$$

are also homotopy equivalences (for all indices $i$ and $j$ ). We will use the same notation $\hat{\phi}_{i}$ and $\hat{\psi}_{i}$ to denote the maps

$$
\begin{aligned}
& \hat{\phi}_{i}: \widetilde{\operatorname{Top}} \mathscr{C}_{i} / \operatorname{Top} \mathscr{C}_{i} \rightarrow \widetilde{\operatorname{Top}} M / \operatorname{Top} M, \\
& \hat{\psi}_{i}: \widetilde{\operatorname{Top}} T_{i} / \operatorname{Top} T_{i} \rightarrow \widetilde{\operatorname{Top}} M / \operatorname{Top} M .
\end{aligned}
$$

Consequently, it suffices in proving Theorem 10.9 to show that the last of these maps $\sum_{i}\left(\hat{\phi}_{i}\right)_{s}+\sum_{i}\left(\hat{\psi}_{i}\right)_{s}$ induces an isomorphism for $0<s \leq(m-10) / 7$ and $m>10$, where $\hat{\phi}_{i}$ and $\hat{\psi}_{i}$ are the maps of (10.16). But this follows from Corollary A.1 (a) of our appendix (which extends the main result of [25]), a stability result due to Igusa [37], and Hatcher's spectral sequence [33, Proposition 2.1]. We proceed to discuss this implication in more detail. 
Let $\sigma: V \rightarrow M$ be the inclusion of an open subset. Then it determines continuous maps

$$
\sigma_{p}: C\left(V \times I^{p}\right) \rightarrow C\left(M \times I^{p}\right), \quad \sigma^{\prime}: \mathscr{P}(V) \rightarrow \mathscr{P}(M),
$$

where $I^{p}$ denotes the $p$-factor Cartesian product $[0,1] \times \cdots \times[0,1], C\left(N \times I^{p}\right)$ denotes the space of all topological pseudo-isotopies on $N \times I^{p}$ with compact support, and $\mathscr{P}(N)$ is the direct limit (as $p \rightarrow+\infty)$ of $C\left(N \times I^{p}\right)$. Igusa shows in [37] that for $q \leq(m-7) / 3$ (recall $m>10)$, one can identify $\pi_{q} C\left(V \times I^{p}\right)$ and $\pi_{q} C\left(M \times I^{p}\right)$ with $\pi_{q} \mathscr{P}(V)$ and $\pi_{q} \mathscr{P}(M)$, respectively, and the map on $\pi_{q}$ determined by $\sigma_{p}$ can be identified with that determined by $\sigma^{\prime}$.

Let $E_{p q}^{r}(V)$ and $E_{p q}^{r}(M)$ be the first quadrant spectral sequences constructed by Hatcher in [33, Proposition 2.1]. Then $\sigma$ induces a mapping between these two spectral sequences. Define a spectral sequence $\mathscr{E}_{p q}^{r}$ by

$$
\mathscr{E}_{p q}^{r}=\left(\bigoplus_{i} E_{p q}^{r}\left(\mathscr{C}_{i}\right)\right) \oplus\left(\bigoplus_{i} E_{p q}^{r}\left(T_{i}\right)\right) .
$$

Then the inclusions $\phi_{i}$ and $\psi_{i}$ induce a map $\Phi_{p q}^{r}$ of this spectral sequence to $E_{p q}^{r}(M)$, which is an isomorphism from $\mathscr{E}_{p q}^{1}$ to $E_{p q}^{1}(M)$, provided $(m-7) / 3 \geq$ $q$. This last assertion is a consequence of Corollary A.1(a), [33, Proposition 2.1], and Igusa's stability result [37] cited above. Theorem 10.9 now follows from this assertion, the following algebraic lemma (whose proof is left to the reader), and the fact that Hatcher's spectral sequence $E_{p q}^{r}(V)$ converges to $\pi_{p+q+1}(\widetilde{\operatorname{Top}} V / \operatorname{Top} V)$ (cf. [33, Proposition 2.1]), which implies that $\Phi_{p q}^{r}$ converges to $\sum_{i}\left(\hat{\phi}_{i}\right)_{p+q+1}+\sum_{i}\left(\hat{\psi}_{i}\right)_{p+q+1}$.

Lemma 10.10. Let $\Phi_{p q}^{s}: \mathscr{E}_{p q}^{s} \rightarrow E_{p q}^{s}$ be a map between first quadrant spectral sequences and $m$ an integer such that $\Phi_{p q}^{1}$ is an isomorphism for all pairs of integers $(p, q)$, where $q \leq m$. Then $\Phi_{p q}^{s}$ is an isomorphism for all $s \geq 1$ (including $s=\infty$ ) provided $p+q \leq m$.

The following qualitative facts about the spaces $G(M), G\left(\mathscr{C}_{i}\right)$, and $G\left(T_{j}\right)$ are easy consequences of Eilenberg obstruction theory (and the Mostow-Prasad rigidity theorem in the case of $\pi_{0}$ of $\left.G(M)\right)$.

Remark 10.4. Provided $n \geq 2, \pi_{n} G(N)=0$, where $N$ is either $M, \mathscr{C}_{i}$, or $T_{j}$. Also, $G\left(T_{j}\right)$ is arc-connected, while $\pi_{1} G\left(T_{j}\right)$ and $\pi_{1} G\left(\mathscr{C}_{i}\right)$ both vanish. If $M$ has finite volume, then $G(M)$ has only a finite number of arc-components and $\pi_{1} G(M)$ vanishes.

To formulate our next result, we introduce some notation. If $N_{i}$ is a sequence of topological manifolds, let $\bigoplus_{i}$ Top $N_{i}$ denote the direct limit, as $j \rightarrow+\infty$, of the finite Cartesian products

$$
\text { Top } N_{1} \times \operatorname{Top} N_{2} \times \cdots \times \operatorname{Top} N_{j} .
$$


If each $N_{i}$ is an open subset of $M$ with inclusion map denoted by $\sigma_{i}$, define a continuous map

$$
\sum \hat{\sigma}_{i}: \bigoplus_{i} \operatorname{Top} N_{i} \rightarrow \operatorname{Top} M
$$

to be the map induced in the limit, as $j \rightarrow+\infty$, of the continuous maps

$$
\left(x_{1}, x_{2}, \ldots, x_{j}\right) \mapsto \hat{\sigma}_{1}\left(x_{1}\right) \circ \hat{\sigma}_{2}\left(x_{2}\right) \circ \cdots \circ \sigma_{j}\left(x_{j}\right),
$$

where $\circ$ denotes composition of functions and $x_{i} \in \operatorname{Top} N_{i}$. If $f: A \rightarrow \operatorname{Top} M$ and $g: B \rightarrow$ Top $M$ are a pair of continuous maps, then

$$
f+g: A \times B \rightarrow \text { Top } M
$$

denotes the composite of $f \times g: A \times B \rightarrow$ Top $M$ with the group multiplication (composition of functions) in Top $M$.

In light of Remark 10.4, Theorem 10.9 has the following consequence concerning the homotopy type of Top $M$.

Corollary 10.11. The continuous map $\left(\sum \hat{\phi}_{i}\right)+\left(\sum \hat{\psi}_{i}\right)$, where

$$
\left(\sum \hat{\phi}_{i}\right)+\left(\sum \hat{\psi}_{i}\right):\left(\bigoplus_{i} \operatorname{Top} \mathscr{C}_{i}\right) \times\left(\bigoplus_{i} \operatorname{Top} T_{i}\right) \rightarrow \operatorname{Top} M,
$$

induces an isomorphism on homotopy groups $\pi_{s}$ provided $1<s \leq(m-4) / 3$ and $m>10$. When $M$ has finite volume and $m>10$ (recall that $m=\operatorname{dim} M$ ), this map also induces an isomorphism on $\pi_{1}$.

The next result extends the above analysis to $\pi_{0}(\operatorname{Top} M)$, when $M$ has finite volume. Let Out $\pi_{1} M$ denote the outer automorphism group of $\pi_{1} M$. Recall that Out $\pi_{1} M$ is in one-to-one correspondence with the homotopy classes of self-homotopy equivalences of $M$. Let Out $\pi_{1} M$ be the subgroup representable by elements in Top $M$; i.e., Out $\pi_{1} M$ is the image of the canonical homomorphism

$$
\text { Top } M \rightarrow \text { Out } \pi_{1} M \text {. }
$$

Let $\operatorname{Top}_{0} M$ denote the kernel of this homomorphism. Note that the values of the map $\left(\sum \hat{\phi}_{i}\right)+\left(\sum \hat{\psi}_{i}\right)$ are in $\operatorname{Top}_{0} M$. The above discussion together with elementary homotopy reasoning and Remark 10.4 shows that Theorem 10.9 and Corollary 10.11 have the following refinement.

Corollary 10.12. Suppose $M$ has finite volume and $m>10$ (recall that $m=$ $\operatorname{dim} M)$. Then Top $M$ is an extension of the finite discrete group Out' $\pi_{1} M$ by the topological group $\operatorname{Top}_{0} M$. Furthermore,

$$
\left(\sum \hat{\phi}_{i}\right)+\left(\sum \hat{\psi}_{i}\right):\left(\bigoplus_{i} \operatorname{Top} \mathscr{C}_{i}\right) \times\left(\bigoplus_{i} \operatorname{Top} T_{i}\right) \rightarrow \operatorname{Top}_{0} M
$$


is a "weak homotopy equivalence through a stable range of dimensions." To be specific, this map induces an isomorphism on the homotopy groups $\pi_{s}$ for $0 \leq s \leq(m-4) / 3$.

Definition 10.0. If $X$ is a compact manifold (with possibly nonempty boundary) and $A$ is a subspace of $X$, then $\operatorname{Homeo}(X, A)$ denotes the space of all self-homeomorphisms of $X$ that restrict to the identity map on $A$. Furthermore, we abbreviate $\operatorname{Homeo}(X, \varnothing)$ by $\operatorname{Homeo} X$. Note that if $\partial X=\varnothing$, then Homeo $X=$ Top $X$.

We next make three remarks that will be used to obtain a more explicit result from Corollary 10.12 .

Remark 10.5. When $M$ has finite volume, it has only a finite number of cusps $\mathscr{C}_{1}, \mathscr{C}_{2}, \ldots, \mathscr{C}_{n}$ (if $M$ is compact, it has no cusps, i.e., $n=0$ ). There exists a sequence $B_{1}, B_{2}, \ldots, B_{n}$ of compact flat Riemannian manifolds such that $\mathscr{C}_{i}=B_{i} \times R$ for $i=1,2, \ldots, n$. Consequently,

$$
\bigoplus_{i} \operatorname{Top} \mathscr{C}_{i}=\underset{i=1}{\times} \operatorname{Top}\left(B_{i} \times R\right)
$$

which contains $\times_{i=1}^{n} \operatorname{Homeo}\left(B_{i} \times I, B_{i} \times \partial I\right)$ as a deformation retract, where $I=[0,1]$ and $\partial I=\{0,1\}$.

Remark 10.6. When $M$ has finite volume, the number of $t$-simple closed geodesics in $M$ is countable infinite. Furthermore, each tube $T_{i}$ is either homeomorphic to $S^{1} \times R^{n-1}$ or to $\mathscr{M}^{2} \times R^{m-2}$, where $\mathscr{M}^{2}$ denotes the (open) Möbius band. In the first case, Top $T_{i}$ contains

$$
\operatorname{Homeo}\left(S^{1} \times D^{m-1}, S^{1} \times \partial D^{m-1}\right)
$$

as a deformation retract and, in the second, it contains

$$
\operatorname{Homeo}\left(\overline{\mathscr{K}} \times D^{m-2}, \partial\left(\overline{\mathscr{M}}^{2} \times D^{m-2}\right)\right)
$$

as a deformation retract, where $\overline{\mathscr{M}}^{2}$ denotes the closed Mobius band, i.e., $\overline{\mathscr{M}}$ is the total space of the nonorientable $I$-bundle over $S^{1}$.

Remark 10.7. When $M$ is compact and $m>2$, Mostow's rigidity theorem implies that the homeomorphism (10.23) is a split epimorphism. In particular, Out' $\pi_{1} M=$ Out $\pi_{1} M$.

Putting these three remarks together with Corollary 10.12 yields the following more explicit result.

Corollary 10.13. Suppose $M$ is compact orientable and $\operatorname{dim} M>10$. Then the spaces $\operatorname{Top} M$ (recall that $\operatorname{Top} M=\operatorname{Homeo} M$ when $M$ is compact) and $\left(\bigoplus \operatorname{Homeo}\left(S^{1} \times D^{m-1}, S^{1} \times \partial D^{m-1}\right)\right) \times \operatorname{Out}\left(\pi_{1} M\right)$ are "weakly homotopically equivalent through a stable range of dimensions." More precisely stated, there is a continuous map of the second space into Top $M$ that induces a bijection on path 
components and an isomorphism on $\pi_{s}$ for each path component provided $1 \leq$ $s \leq(m-4) / 3$ (recall that $m=\operatorname{dim} M)$. Here, the direct limit, as $j \rightarrow+\infty$, of the Cartesian product of $j$-copies of the space $\operatorname{Homeo}\left(S^{1} \times D^{m-1}, S^{1} \times \partial D^{m-1}\right)$ is denoted by $\bigoplus \operatorname{Homeo}\left(S^{1} \times D^{m-1}, S^{1} \times \partial D^{m-1}\right)$, and the finite group $\operatorname{Out}\left(\pi_{1} M\right)$ is equipped with the discrete topology.

Let $\hat{\pi}_{1} M$ denote the set of conjugacy classes of pairs $\left\{\gamma, \gamma^{-1}\right\}$ of indivisible elements in $\pi_{1} M$. (Recall that $\gamma \in \pi_{1} M$ is divisible provided there exists an element $\alpha \in \pi_{1} M$ and an integer $n>1$ such that $\alpha^{n}=\gamma$. In particular, the identity element is divisible.)

Remark 10.8. Since the tubes $T_{i}$ are pairwise disjoint, the topological group $\bigoplus$ Homeo $\left(S^{1} \times D^{m-1}, S^{1} \times \partial D^{m-1}\right)$ defined in Corollary 10.13 is a subgroup of $\operatorname{Top}_{0} M$. Furthermore, it is isomorphic to the topological group of all functions from $\hat{\pi}_{1} M$ to $\operatorname{Homeo}\left(S^{1} \times D^{m-1}, S^{1} \times \partial D^{m-1}\right)$ with finite support and pointwise multiplication.

We continue with our assumptions that $M$ has finite volume and $m>10$. Let $\bar{M}$ denote the Gromov-Margulis manifold compactification of $M$; cf. [32]. Let $\operatorname{Homeo}(\bar{M})_{0}$ denote the kernel of the natural homomorphism

$$
\operatorname{Homeo}(\bar{M}) \rightarrow \text { Out } \pi_{1} M \text {. }
$$

Mostow's rigidity theorem as extended by Gopal Prasad from the compact to the finite volume situation; cf. [51, Theorem 24.2], yields that this homomorphism is a split epimorphism; i.e.,

$$
\text { Homeo } \bar{M}=\operatorname{Homeo}(\bar{M})_{0} \rtimes \operatorname{Out}\left(\pi_{1} M\right),
$$

and $\operatorname{Out}\left(\pi_{1} M\right)$ is a finite group. Our next result gives an analysis, in a stable range of dimensions, of the homotopy type of $\operatorname{Homeo}(\bar{M})_{0}$.

Corollary 10.14. When $M$ has finite volume (and $\operatorname{dim} M>10$ ), the spaces Homeo $(\bar{M})_{0}$ and $\left(\bigoplus_{i} \operatorname{Top} T_{i}\right) \times\left(\times_{i=1}^{n} \mathscr{P}\left(B_{i}\right)\right)$ have the same "weak homotopy type through a stable range of dimensions." More precisely, there exist continuous maps $f$ and $g$ with range the first and second spaces above, respectively, and the same domain. These maps induce isomorphisms on $\pi_{s}$ for $s \leq(m-7) / 3$ (recall that $m=\operatorname{dim} M$ ). Furthermore, the common domain of both $f$ and $g$ is

$$
\left(\bigoplus_{i} \operatorname{Top} T_{i}\right) \times\left(\stackrel{\times}{x}_{i=1}^{n} \operatorname{Homeo}\left(B_{i} \times[0,1], B_{i} \times 1\right)\right) ;
$$

cf. Remark 10.6 regarding the homotopy type of $\bigoplus_{i}$ Top $T_{i}$.

Proof. Consider the following two fibrations

$$
\begin{gathered}
\text { Homeo }(\bar{M}, \partial \bar{M})_{0} \rightarrow \operatorname{Homeo}(\bar{M})_{0} \rightarrow \underset{i=1}{\times} \operatorname{Top}_{0}\left(B_{i}\right), \\
F \times E_{1} \rightarrow E_{0} \times E_{1} \rightarrow \underset{i=1}{\times} \operatorname{Top}_{0}\left(B_{i}\right),
\end{gathered}
$$


where

$$
\begin{aligned}
E_{0} & =\stackrel{\times}{i=1}_{i}^{n} \operatorname{Homeo}\left(B_{i} \times[0,1], B_{i} \times 1\right), \quad E_{1}=\bigoplus_{i} \operatorname{Top} T_{i}, \quad \text { and } \\
F & ={ }_{i=1}^{n} \operatorname{Homeo}\left(B_{i} \times[0,1], B_{i} \times\{0,1\}\right) .
\end{aligned}
$$

The projection maps in these fibrations are epimorphisms because of the main result in [21]. Consider the subspace of $\operatorname{Homeo}(\bar{M}, \partial \bar{M})_{0}$ consisting of all homeomorphisms that are the identity in the complement of a compact subset of $\bar{M}-\partial \bar{M}$. This space can be identified with Top $_{0}(M)$. Similarly, there is a subspace $F_{0}$ of $F$ that we identify with $\bigoplus_{i}$ Top $\mathscr{C}_{i}$. There is a natural map $f$ of the second fibration of (10.26) into the first that covers the identity map on the base spaces. On fibers, $f$ restricts to the map $\left(\sum \hat{\phi}_{i}\right)+\left(\sum \hat{\psi}_{i}\right)$ of Corollary 10.12 in the subspace $F_{0} \times E_{1}$ of $F \times E_{1}$ to the subspace $\operatorname{Top}_{0}(M)$ of $\operatorname{Homeo}(\bar{M}, \partial M)_{0}$. But both of these subspaces are deformation retracts. Hence, Corollary 10.12 implies that $f$ induces an isomorphism on $\pi_{s}$ for all $s \leq(m-4) / 3$. Let $g=\mathrm{id} \times\left(\times_{i=1}^{n} \tau_{i}\right)$, where

$$
\tau_{i}: \operatorname{Homeo}\left(B_{i} \times[0,1], B_{i} \times 1\right) \rightarrow \mathscr{P}\left(B_{i}\right)
$$

is the map in the direct limit. Because of Igusa's stability result [37], $g$ induces an isomorphism on $\pi_{s}$ for all $s \leq(m-7) / 3$. This completes the proof of Corollary 10.14 .

In light of Corollaries $10.11-10.14$, it is clearly important to have homotopy information about both $\operatorname{Top} \mathscr{C}_{i}$ and $\operatorname{Top} T_{j}$. The following is a recollection of previously known results.

Lemma 10.15. Let $E$ be the total space of a vector bundle whose base space is a compact connected flat Riemannian manifold $B$, and let $\operatorname{dim} E=m-1$, where $m>10$. Then

$$
\begin{aligned}
& \pi_{s} \operatorname{Top}(E \times R) \otimes Q=0, \text { for } 1 \leq s \leq(m-4) / 3 ; \\
& \pi_{s} \mathscr{P}(B) \otimes Q=0, \text { for all nonnegative integers } s ; \text { and } \\
& \pi_{0} \operatorname{Top}(E \times R) \text { is a countably infinite abelian group of exponent } \\
& 2, \text { when } B \text { is the circle } S^{1} .
\end{aligned}
$$

(Here $Q$ denotes the additive group of rational numbers.)

Proof. Calculations one and two are implicit in [17], and calculation three is implicit in [33, Theorem 4.1-Remark 2]. More explicitly, the second calculation is a consequence of a basic result of Waldhausen's (cf. [33, Corollary 7.3]) and [17, Theorem 3.1]. The first calculation uses, in addition to those facts, Hatcher's spectral sequence [33, Proposition 2.2] and the Main Theorem of [15]. The third calculation also requires the use of a basic result of Hatcher and Wagoner (cf. [33, Theorem 3.1]).

Lemma 10.15 now allows us to deduce the following specific information about the homotopy groups of Top $M$ and Homeo $\bar{M}$ from Corollaries 10.1110.14. (Again, $\bar{M}$ denotes the Gromov-Margulis manifold compactification of M.) 
Corollary 10.16. Assume that $\operatorname{dim} M>10$ (recall that $m=\operatorname{dim} M$ ). Then $\pi_{s}$ Top $M \otimes Q=0$ provided $2 \leq s \leq(m-4) / 3$. If, in addition, we assume that $M$ has finite volume, then we also have

(i) $\pi_{1} \operatorname{Top} M \otimes Q=0$;

(ii) neither $\pi_{0} \operatorname{Top} M$ nor $\pi_{0}$ Homeo $\bar{M}$ is finitely generated;

(iii) $\pi_{s}$ Homeo $\bar{M} \otimes Q=0$ provided $1 \leq s \leq(m-7) / 3$.

When $M$ is compact, we have the additional information that $\pi_{0}$ Top $M$ is isomorphic to a semidirect product $\mathbf{Z}_{2}^{\infty} \rtimes$ Out $\pi_{1} M$ where $\mathbf{Z}_{2}^{\infty}$ denotes the countably infinite abelian group of exponent 2 (recall that Out $\pi_{1} M$ is a finite group).

Let Diff $M$ denote the group of all diffeomorphisms of $M$ with compact support. Our next application of Theorem 9.5 uses the general theory in [17, Theorem 4.5] (which depends on Waldhausen's foundational work in [63]) together with [25, Corollary 7.2] and Igusa's improved stable range [37].

Corollary 10.17. If $M$ is compact, $\operatorname{dim} M>10$, and $s$ is an integer satisfying $1 \leq s \leq(m-4) / 3$ (recall that $m=\operatorname{dim} M)$, then

$$
\pi_{s} \operatorname{Diff} M \otimes Q=\left\{\begin{array}{l}
\bigoplus_{j=1}^{\infty} H_{(s+1)-4 j}(M, Q), \quad \text { if } m \text { is odd }, \\
0, \quad \text { if } m \text { is even. }
\end{array}\right.
$$

We end this rather long section with a brief discussion of two methods for obtaining more precise calculations of $\pi_{s} \operatorname{Diff} M$, in a stable range of dimensions $s$. First we note that the method used in Theorem 10.9 to analyze Top $M$ can also be used on Diff $M$. For instance, Hatcher's spectral sequence $E_{p q}^{r}$ in [33, Proposition 2.2] converges to $\pi_{p+q+1}(\widetilde{\operatorname{Diff}} M / \operatorname{Diff} M)$. Since we can analyze $E_{p q}^{2}$ using Theorem A.2 of our appendix together with the fact that $E_{p q}^{2}=H_{p}\left(\mathbf{Z}_{2} ; \mathscr{P}_{\text {Diff }} M\right)$ provided $(m-7) / 3 \geq q$, this spectral sequence is useful. Also, the homotopy groups of $G(M) / \widetilde{\mathrm{Diff}} M$ can be examined via surgery theory using Theorem 9.5. Putting this information together with the facts about $G(M)$ already mentioned in Remark 10.4 is the first method for analyzing Diff $M$. (We note that this method can be used to prove Corollary 10.17.)

The second method relates $\pi_{s}$ Diff $M$ more directly to $\pi_{s}$ Top $M$. In fact, it can be shown, through a stable range of dimensions $s$, that

$$
\pi_{s} \operatorname{Diff} M \simeq \pi_{s} \text { Top } M \oplus \pi_{s+1}(\operatorname{Top} M / \operatorname{Diff} M) .
$$

(10.28) is obtained by showing that the inclusion map

$$
\tau: \text { Diff } M \rightarrow \text { Top } M
$$

is (stably) a "homotopically split surjection"; i.e., there exists a space $K$ and a pair of continuous maps $f: K \rightarrow \operatorname{Diff} M$ and $g: K \rightarrow \operatorname{Top} M$ such that

(i) the composite $\tau \circ f$ is homotopic to $g$, and

(ii) $g$ induces an isomorphism on $\pi_{s}$ for a stable range of integers $s$. 
Because of Theorem 10.9, it suffices to do this splitting when $M$ is replaced by $\mathscr{C}_{i}$ or $T_{j}$, i.e., by a (high dimensional) manifold $N$ that is the total space of a vector bundle whose base is a compact (connected) flat Riemannian manifold. In this situation, the splitting is accomplished by using Theorem A.2 of our appendix (which is the psuedo-isotopy version of $(10.28)$ ) together with Hatcher's spectral sequence [33, Proposition 2.1]. The ideas in the proof of Theorem A.2 are also used. In particular, we consider the subgroup of Diff $M$ consisting of all "controlled" diffeomorphisms (and a Hatcher-type spectral sequence for these). By covering space arguments, peculiar to flat Riemannian manifold, we define a map from $\operatorname{Diff} N$ to this subgroup. Also, we use the fact that $G(N) / \widetilde{\operatorname{Top}} N$ is a contractible space, which is a consequence of [21]. Finally, work of Burghelea and Lashof (cf. [10, Theorem 1.1]) shows that $\pi_{*+1}(\operatorname{Top} M / \operatorname{Diff} M)$ is a "sort of homology theory" whose coefficients are $\pi_{*} \mathscr{P}_{\text {Diff }}$ (point). Important information concerning $\pi_{i} \mathscr{P}_{\text {Diff }}(*)$ can be deduced from Waldhausen's work [63]; e.g., using it and [5], it was shown in [17] that

$$
\pi_{i} \mathscr{P}_{\text {Diff }}(*) \otimes Q= \begin{cases}Q, & \text { if } i \equiv 3 \bmod 4 \\ 0, & \text { otherwise }\end{cases}
$$

\section{APPENDIX}

In this appendix, we let $M$ denote (unless otherwise specified) any real, complex, quaternionic or Cayley complete hyperbolic manifold. For any smooth manifold $X$, we let $\mathscr{P}(X)$ and $\mathscr{P}_{\text {Diff }}(X)$ denote the semisimplical space of stable topological pseudo-isotopies on $X$ with compact support and the semisimplical space of stable smooth pseudo-isotopies on $X$ with compact support.

In Corollary A.1(a), we determine the homotopy type of space $\mathscr{P}(M)$. The authors announced Corollaries A.1(a)-(c) in $[23,25]$ and proved them in [23, 25] for any $M$ of finite volume. Theorem A.1 was announced by the authors in [22] for any compact $M$.

In Theorem A.2, we determine the homotopy type of the space $\mathscr{P}_{\text {Diff }}(M)$ for any real complete hyperbolic manifold $M$. The authors announced Theorem A.2 in [22] for any compact Riemannian manifold of strictly negative curvature.

Some $\Omega$-spectra. For each $n \geq 0$ and any smooth manifold $X$, we denote by $\mathscr{P}_{\text {Diff }, b}\left(X \times R^{n}\right)$ the space of all stable smooth pseudo-isotopies on $X \times R^{n}$ that have compact support in the $X$-factor and that are bounded in the $R^{n}$ factor, and we denote by $\mathscr{P}_{b}\left(X \times R^{n}\right)$ the space of all stable topological pseudoisotopies on $X \times R^{n}$ that have compact support in the $X$-factor and that are bounded in the $R^{n}$-factor. The loop spaces $\Omega \mathscr{P}_{\text {Diff, } b}\left(X \times R^{n}\right)$ and $\Omega \mathscr{P}_{b}\left(X \times R^{n}\right)$ are homotopy equivalent to $\mathscr{P}_{\text {Diff }, b}\left(X \times R^{n-1}\right)$ and $\mathscr{P}_{b}\left(X \times R^{n-1}\right)$ for all $n \geq 1$ (cf. [33]). Thus, we may define $\Omega$-spectra $\mathscr{D}(X)$ and $\mathscr{T}(X)$ as follows. Set $\mathscr{D}_{n}(X)=\mathscr{P}_{\text {Diff }, b}\left(X \times R^{n}\right)$ for each $n \geq 0$, and set $\mathscr{D}_{-n}(X)=\Omega^{n} \mathscr{P}_{\text {Diff }}(X)$ for each $n \geq 1$. Set $\mathscr{T}_{n}(X)=\mathscr{P}_{b}\left(X \times R^{n}\right)$ for each $n \geq 0$, and set $\mathscr{T}_{-n}(X)=$ $\Omega^{n} \mathscr{P}_{b}(X)$ for each $n \geq 1$. 
We may define the "homology $\Omega$-spectrum" for $\mathscr{D}()$ over $X$, denoted $\mathbf{H}(X ; \mathscr{D}(*))$, as follows. Let $d_{i} \in \mathscr{D}_{i}(*)$ be the points corresponding to the identity pseudo-isotopies. Then set

$$
\mathbf{H}_{k}(X ; \mathscr{D}(*))=\lim _{i \rightarrow \infty} \Omega^{i}\left(\left(X \times \mathscr{D}_{i+k}(*)\right) /\left(X \times d_{i+k}\right)\right) .
$$

Here $*$ indicates the space with one point, and " $\lim _{i \rightarrow \infty}$ " indicates the direct limit of spaces.

There is a natural map of $\Omega$-spectra $A: \mathbf{H}(X ; \mathscr{D}(*)) \rightarrow \mathscr{D}(X)$ called the amalgamation map. At the $k$ th level, $A$ is defined to be the composite

$$
\lim _{i \rightarrow \infty} \Omega^{i}\left(\left(X \times \mathscr{D}_{i+k}(*)\right) /\left(X \times d_{i+k}\right)\right) \stackrel{\psi}{\rightarrow} \lim _{i \rightarrow \infty} \Omega^{i} \mathscr{D}_{i+k}(X) \cong \mathscr{D}_{k}(X),
$$

where $\psi$ is induced by the composite map $X \times \mathscr{D}(*) \stackrel{\text { proj }}{\longrightarrow} \mathscr{D}(*) \stackrel{\text { inclusion }}{\rightarrow} \mathscr{D}(X)$.

We denote by $N \mathscr{D}(X)$ the cokernel $\Omega$-spectrum for the amalgamation map $A: \mathbf{H}(X, \mathscr{D}(*)) \rightarrow \mathscr{D}(X)$.

Cusps and closed geodesics. Let $\mathscr{C}_{1}, \mathscr{C}_{2}, \mathscr{C}_{3}, \ldots$ denote the cusps of $M$. Each cusp $\mathscr{C}_{i}$ has a tubular neighborhold in $M$ that is diffeomorphic to a real vector bundle over a compact closed aspherical manifold $C_{i}$ that has a virtually poly- $Z$ fundamental group (see [3]). Let $f_{i}: C_{i} \rightarrow M$ denote the zero section map for this vector bundle. Let $g_{i}: S^{1} \rightarrow M$ be a sequence of smooth immersions from the circle into $M$ such that the images $g_{1}\left(S^{1}\right), g_{2}\left(S^{1}\right)$, $g_{3}\left(S^{1}\right), \ldots$ are an enumeration of the set of all $t$-simple closed geodesics in $M$. Let $\left(\bigcup_{i} C_{i}\right) \cup\left(\bigcup_{i} S^{1}\right)$ denote the disjoint union of all the $\left\{C_{i}\right\}$ with all the \{domain $\left(g_{i}\right)$ \}. Let $I:\left(\bigcup_{i} C_{i}\right) \cup\left(\bigcup_{i} S^{1}\right) \rightarrow M$ denote the map that, when restricted to any $C_{i}$, is equal to $f_{i}$ and, when restricted to the $i$ th copy of $S^{1}$, is equal to $g_{i}$.

Theorem A.1. Let $M$ be any real, complex, quaternionic, or Cayley complete hyperbolic manifold. The map $I:\left(\bigcup_{i} C_{i}\right) \cup\left(\bigcup_{i} S^{1}\right) \rightarrow M$ induces an equivalence of $\Omega$-spectra

$$
\mathscr{T}\left(\left(\bigcup_{i} C_{i}\right) \cup\left(\bigcup_{i} S^{1}\right)\right) \cong \mathscr{T}(M) .
$$

We have the following corollaries of Theorem A.1.

The equivalence of Theorem A.1 at the zeroth levels of the $\Omega$-spectra becomes the following.

Corollary A.1(a). The map I: $\left(\bigcup_{i} C_{i}\right) \cup\left(\bigcup_{i} S^{1}\right) \rightarrow M$ induces a homotopy equivalence of spaces $\mathscr{P}\left(\left(\bigcup_{i} C_{i}\right) \cup\left(\bigcup_{i} S^{1}\right)\right) \cong \mathscr{P}(M)$.

The first $k$ homotopy groups of $\mathscr{T}_{k}(X)$ are $K_{-k+2}\left(Z \pi_{1} X\right), \ldots, K_{-1}\left(Z \pi_{1} X\right)$, $\widetilde{K}_{0}\left(Z \pi_{1} X\right), \mathrm{Wh}\left(\pi_{1}(X)\right)$ for any manifold $X$ (cf. [1]). If $X$ is a compact closed aspherical manifold having a virtually poly- $Z$ fundamental group, then all these homotopy groups are zero (cf. [19]). So by the equivalence of Theorem A.1 we have the following. 
Corollary A.1(b). $\mathrm{Wh}\left(\pi_{1} M\right)=0, \widetilde{K}_{0}\left(Z \pi_{1} M\right)=0, K_{n}\left(Z \pi_{1} M\right)=0$ for all $n<0$.

Using only the equivalence of Corollary A.1(a), we can argue as in [25] to show the following.

Corollary A.1(c). (i) For any integer $n \geq 2$, we have that $\mathrm{Wh}_{n}\left(\pi_{1}(M)\right) \otimes Z(1 / N)$ $=0$, where $N=[(n+1) / 2]$ !.

(ii) For any integer $n$, we have that $K_{n}\left(Z \pi_{1}(M)\right) \otimes Q=H_{n}(M, Q) \oplus$ $\left(\bigoplus_{i=1}^{\infty} H_{n-1-4 i}(M, Q)\right)$.

Theorem A.2. Let $M$ be any real complete hyperbolic manifold. There are the following homotopy equivalences of spaces.

(a) $\mathscr{P}_{\text {Diff }}(M) \cong \mathbf{H}_{0}(M ; \mathscr{D}(*)) \times N \mathscr{D}_{0}(M)$.

(b) $N \mathscr{D}_{0}(M) \cong N \mathscr{D}_{0}\left(\left(\bigcup_{i} C_{i}\right) \cup\left(\bigcup_{i} S^{1}\right)\right)$.

(c) $N \mathscr{D}_{0}\left(\left(\bigcup_{i} C_{i}\right) \cup\left(\bigcup_{i} S^{1}\right)\right)=\mathscr{P}\left(\left(\bigcup_{i} C_{i}\right) \cup\left(\bigcup_{i} S^{1}\right)\right)$.

Remark A.2. The equivalence of Corollary A.2(a) is the Cartesian product of the projection map $\mathscr{P}_{\text {Diff }}(M) \rightarrow N \mathscr{D}_{0}(M)$ with a homotopy left inverse of the amalgamation map. The equivalence of Theorem A.2(b) is induced by the map I: $\left(\bigcup_{i} C_{i}\right) \cup\left(\bigcup_{i} S^{1}\right) \rightarrow M$. The equivalence of Theorem A.2(c) is induced by the forgetful map $\mathscr{D}\left(\left(\bigcup_{i} C_{i}\right) \cup\left(\bigcup_{i} S^{1}\right)\right) \rightarrow \mathscr{T}\left(\left(\bigcup_{i} C_{i}\right) \cup\left(\bigcup_{i} S^{1}\right)\right)$.

Proof of Theorem A.1. The proof is similar to the argument given in [25], so we will be brief. Let $J: \mathscr{T}\left(\left(\bigcup_{i} C_{i}\right) \cup\left(\bigcup_{i} S^{1}\right)\right) \rightarrow \mathscr{T}(M)$ denote the map of $\Omega$-spectra induced by the map $I:\left(\bigcup_{i} C_{i}\right) \cup\left(\bigcup_{i} S^{1}\right) \rightarrow M$, and for each positive integer $n$, let $J^{n}: \mathscr{T}\left(\left(\bigcup_{i=1}^{n} C_{i}\right) \cup\left(\bigcup_{i=1}^{n} S^{1}\right)\right) \rightarrow \mathscr{T}(M)$ denote the map of $\Omega$ spectra induced by the map $I \mid\left(\left(\bigcup_{i=1}^{n} C_{i}\right) \cup\left(\bigcup_{i=1}^{n} S^{1}\right)\right)$. It is convenient to divide the proof into the following two steps.

Step I. In this step, we show that for each positive integer $k$ the map $J$ : $\mathscr{T}_{k}\left(\left(\bigcup_{i} C_{i}\right) \cup\left(\bigcup_{i} S^{1}\right)\right) \rightarrow \mathscr{T}_{k}(M)$ induces an injection on all the homotopy groups.

To show this, it will suffice to construct, for each positive integer $n$, a left homotopy inverse for the map

$$
J^{n}: \mathscr{T}_{k}\left(\left(\bigcup_{i=1}^{n} C_{i}\right) \cup\left(\bigcup_{i=1}^{n} S^{1}\right)\right) \rightarrow \mathscr{T}_{k}(M),
$$

which we will denote by

$$
J_{n}: \mathscr{T}_{k}(M) \rightarrow \mathscr{T}_{k}\left(\left(\bigcup_{i=1}^{n} C_{i}\right) \cup\left(\bigcup_{i=1}^{n} S^{2}\right)\right)
$$

The construction of $J_{n}$ is similar to the constructions used in [25,§2].

Recall that for each cusp $\mathscr{C}_{i}$ of $M$ there is a covering space projection $p_{i}: N_{i} \times R \rightarrow M$ that satisfies the following (see [3]). 
A.3. (a) $N_{i}$ is a smooth manifold that is the total space of a real vector bundle over the compact closed aspherical manifold $C_{i}$ having a virtually poly$Z$ fundamental group.

(b) The restricted map $p_{i}: N_{i} \times(-\infty, 0] \rightarrow M$ is an embedding whose image is equal to a tubular neighborhood of the cusp $\mathscr{C}_{i}$ in $M$.

(c) For each $y \in N_{i}$, the path $f: R \rightarrow M$ defined by $f(t)=p_{i}(y, r(t))$ is a unit speed geodesic in $M$, where $r: R \rightarrow R$ is a diffeomorphism independent of $y$.

(d) Given any $\varepsilon>0$, there is $t>0$ such that for each vector $v \in$ $\operatorname{Tan}\left(N_{i} \times(t, \infty)\right)$ we must have that

$$
\langle v, v\rangle_{N_{i} \times R} \leq \varepsilon\left\langle d p_{i}(v), d p_{i}(v)\right\rangle_{M},
$$

where $\langle,\rangle_{N_{i} \times R}$ denotes the product Riemann metric on $N_{i} \times R$ and $\langle,\rangle_{M}$ is the given metric on $M$.

We can define a map $r_{i}: \mathscr{T}_{k}(M) \rightarrow \mathscr{T}_{k}\left(C_{i}\right)$ as follows. Let

$$
f:\left(M \times R^{k} \times I^{u}\right) \times I \rightarrow\left(M \times R^{k} \times I^{u}\right) \times I
$$

represent a point of $\mathscr{T}_{k}(M)$. Recall that $f$ is compactly supported in the $M$ factor and is bounded in the $R^{k}$-factor. Pull the pseudo-isotopy $f$ back along the covering projection $p_{i}: N_{i} \times R \rightarrow M$ to a pseudo-isotopy

$$
\hat{f}:\left(N_{i} \times R \times R^{k} \times I^{u}\right) \times I \rightarrow\left(N_{i} \times R \times R^{k} \times I^{u}\right) \times I .
$$

Note that A.3(a) allows us to extend the bundle $N_{i} \rightarrow C_{i}$ to a bundle $\bar{N}_{i} \rightarrow C_{i}$ that has the $x_{i}$-dimensional disc for fiber and such that each fiber of $N_{i} \rightarrow C_{i}$ is the interior of the corresponding disc fiber of $\bar{N}_{i} \rightarrow C_{i}$. We denote the two point compactification of $R$ by $\bar{R}$. Note that $\bar{R}$ is a compact interval with boundary points $\partial_{ \pm} \bar{R}$ corresponding to $\pm \infty$. Thus, we have the compactification $\bar{N}_{i} \times \bar{R}$ of $N_{i} \times R$. Since $f$ has compact support in the $M$-factor, it follows from A.3(b) and (c) that for any $t>0$ the support of $\hat{f}$ intersected with $\left(N_{i} \times(-\infty, t] \times R^{k} \times I^{u}\right) \times I$ is a compact set in the factor $N_{i} \times(-\infty, t]$. So we may extend $\hat{f}$ to a homeomorphism

$$
\begin{aligned}
& \hat{f}:\left(\left(\bar{N}_{i}-N_{i}\right) \times \bar{R} \cup \bar{N}_{i} \times\left(\bar{R}-\partial_{+} \bar{R}\right)\right) \times R^{k} \times I^{u} \times I \\
& \quad \rightarrow\left(\left(\bar{N}_{i}-N\right) \times \bar{R} \cup \bar{N}_{i} \times\left(\bar{R}-\partial_{+} \bar{R}\right)\right) \times R^{k} \times I^{u} \times I
\end{aligned}
$$

by letting $\hat{f}$ be the identity on $\left(\left(\bar{N}_{i}-N_{i}\right) \times \bar{R} \cup \bar{N}_{i} \times \partial_{-} \bar{R}\right) \times R^{k} \times I^{u} \times I$. For the moment, let us assume that $\hat{f}$ satisfies the following property.

A.4. $\lim _{t \rightarrow \infty} d(y, \hat{f}(y))=0$ uniformly in $y \in\left(N_{i} \times(t, \infty) \times R^{k} \times I^{u}\right) \times I$, where $d($,$) is the product metric on \left(N_{i} \times R \times R^{k} \times I^{u}\right) \times I$.

Then we can extend $\hat{f}$ to a map $\bar{f}$ on $\left(\bar{N}_{i} \times \bar{R} \times R^{k} \times I^{u}\right) \times I$ by letting $\bar{f}$ equal the identity on $\left(\bar{N}_{i} \times \partial_{+} \bar{R} \times R^{k} \times I^{u}\right) \times I$ as well. Note that the bundle projection $\bar{N}_{i} \rightarrow C_{i}$ induces a homotopy equivalence $\mathscr{T}_{k}\left(\bar{N}_{i}\right) \cong \mathscr{T}_{k}\left(C_{i}\right)$, and 
note that the extension $\bar{f}:\left(\bar{N}_{i} \times \bar{R} \times R^{k} \times I^{u}\right) \times I \rightarrow\left(\bar{N}_{i} \times \bar{R} \times R^{k} \times I^{u}\right) \times I$ represents a point in $\mathscr{T}_{k}\left(\bar{N}_{i}\right)$. Thus, we may define $r_{i}: \mathscr{T}_{k}(M) \rightarrow \mathscr{T}_{k}\left(C_{i}\right)$ by $r_{i}(f)=\bar{f}$.

If, in the preceding paragraph, A. 4 does not hold, then we must modify $\hat{f}$ somewhat before we can extend it to

$$
\bar{f}:\left(\bar{N}_{i} \times \bar{R} \times R^{k} \times I^{u}\right) \times I \rightarrow\left(\bar{N}_{i} \times \bar{R} \times R^{k} \times I^{u}\right) \times I .
$$

Note that it follows from A.3(d) that A.4 is always true in the $\left(N_{i} \times R\right)$-factor of $\left(N_{i} \times R \times R^{k} \times I^{u}\right) \times I$. However, A.4 may fail in the factors $R^{k}, I^{u}$, and/or $I$. There will be no loss of generality if we assume that $f$ is the identity map near $\left(M \times R^{k} \times \partial I^{u}\right) \times I \cup\left(M \times R^{k} \times I^{u}\right) \times 0$. So $\hat{f}$ is also the identity map near $\left(N_{i} \times R \times R^{k} \times \partial I^{u}\right) \times I \cup\left(N_{i} \times R \times R^{k} \times I^{u}\right) \times 0$. Choose flows $u_{t}: I \rightarrow I$, $v_{t}: I^{u} \rightarrow I^{u}, w_{t}: R^{k} \rightarrow R^{k}, t \in[0, \infty)$, that satisfy the following properties.

A.5. (a) Each of $u_{t}\left|\partial I, v_{t}\right| \partial I^{u}$ is the identity map. Each of $u_{0}, v_{0}$ is the identity map.

(b) $u_{t}: I \rightarrow I, t \in[0, \infty)$, flows all points except 0 towards the point 1 . $v_{t}: I^{u} \rightarrow I^{u}, t \in[0, \infty)$, flows all the points of $I^{u}-\partial I^{u}$ towards the center point of $I^{u}$.

(c) For each $y \in R^{k}$ and each $t \in[0, \infty)$, we have that $w_{t}(y)=(1 /(1+t)) y$. Define a homeomorphism $h:\left(N_{i} \times R \times R^{k} \times I^{u}\right) \times I \rightarrow\left(N_{i} \times R \times R^{k} \times I^{u}\right) \times I$ as follows.

(d) For each $y \in N_{i}, t \in R, s_{1} \in R^{k}, s_{2} \in I^{u}, s_{3} \in I$ set

$$
h\left(y, t, s_{1}, s_{2}, s_{3}\right)= \begin{cases}\left(y, t, s_{1}, s_{2}, s_{3}\right), & \text { if } t \leq 0, \\ \left(y, t, w_{t}\left(s_{1}\right), v_{t}\left(s_{2}\right), u_{t}\left(s_{3}\right)\right), & \text { if } t \geq 0 .\end{cases}
$$

Note that if we modify $\hat{f}$ by conjugating it with $h$, then the new $\hat{f}$ (i.e., the map $h \hat{f} \circ h^{-1}$ ) will satisfy A.4. Now we may proceed as in the previous paragraph to get the extension $\bar{f}$ of the new $\hat{f}$ and then to define $r_{i}(f)=\bar{f}$.

Recall that for each of the closed geodesics $g_{i}: S^{1} \rightarrow M$ of $M$ there is a covering projection $q_{i}: S^{1} \times R^{m-1} \rightarrow M$ satisfying the following properties.

A.6. (a) $q_{i}(y, 0)=g_{i}(y)$ holds for all $y \in S^{1}$.

(b) Given $\varepsilon>0$, there is $t>0$ such that if $v \in \operatorname{Tan}\left(S^{1} \times R_{t}^{m-1}\right)$, where $R_{t}^{m-1}$ denotes all points $p \in R^{m-1}$ satisfying $|p| \geq t$, then we must have that $\langle v, v\rangle \leq \varepsilon\left\langle d q_{i}(v), d q_{i}(v)\right\rangle_{M}$, where $\langle$,$\rangle is the product Riemannian structure$ on $S^{1} \times R^{m-1}$.

We can now define a map $s_{i}: \mathscr{T}_{k}(M) \rightarrow \mathscr{T}_{k}\left(S^{1}\right)$ as follows. Let

$$
f:\left(M \times R^{k} \times I^{u}\right) \times I \rightarrow\left(M \times R^{k} \times I^{u}\right) \times I
$$

represent a point of $\mathscr{T}_{k}(M)$. Pull the pseudo-isotopy $f$ back along the covering projection $q_{i}: S^{1} \times R^{m-1} \rightarrow M$ to get a pseudo-isotopy

$$
\check{f}:\left(S^{1} \times R^{m-1} \times R^{k} \times I^{u}\right) \times I \rightarrow\left(S^{1} \times R^{m-1} \times R^{k} \times I^{u}\right) \times I .
$$


Define $h^{\prime}:\left(S^{1} \times R^{m-1} \times R^{k} \times I^{u}\right) \times I \rightarrow\left(S^{1} \times R^{m-1} \times R^{k} \times I^{u}\right) \times I$ as follows.

A.7. For each $y \in S^{1}, p \in R^{m-1}, s_{1} \in R^{k}, s_{2} \in I^{u}, s_{3} \in I$, set

$$
h^{\prime}\left(y, p, s_{1}, s_{2}, s_{3}\right)=\left(y, p, w_{t}\left(s_{1}\right), v_{t}\left(s_{2}\right), u_{t}\left(s_{3}\right)\right) \text {, }
$$

where $t=|p|$ and $u_{t}, v_{t}, w_{t}$ comes from A.5. Note that if we modify $\check{f}$ by conjugating it with $h^{\prime}$, then it will follow from A.6(b), A.7, and A.5 that the new $\check{f}$ (i.e., the map $h^{\prime} \circ \check{f} \circ\left(h^{\prime}\right)^{-1}$ ) will satisfy the following property.

A.8. $\lim _{t \rightarrow \infty} d(y, \check{f}(y))=0$ uniformly in $y \in\left(S^{1} \times R_{t}^{m-1} \times R^{k} \times I^{u}\right) \times I$, where $d($,$) denotes the product metric on S^{1} \times R^{m-1} \times R^{k} \times I^{u} \times I$. Thus, we may now extend $\check{f}$ to a pseudo-isotopy $f:\left(S^{1} \times \bar{R}^{m-1} \times R^{k} \times I^{u}\right) \times I \rightarrow$ $\left(S^{1} \times \bar{R}^{m-1} \times R^{k} \times I^{u}\right) \times I$ by setting $\underline{f}$ equal to the identity on $\left(S^{1} \times \partial \bar{R}^{m-1} \times R^{k} \times\right.$ $\left.I^{u}\right) \times I$. Here $\bar{R}^{m-1}$ denotes a disc compactification of $R^{m-1}$, and $\partial \bar{R}^{m-1}$ is the manifold boundary. Finally, we can define $s_{i}: \mathscr{T}_{k}(M) \rightarrow \mathscr{T}_{k}\left(S^{1}\right)$ by setting $s_{i}(f)=\underline{f}$.

Note that $\mathscr{T}_{k}\left(\left(\bigcup_{i=1}^{n} C_{i}\right) \cup\left(\bigcup_{i=1}^{n} S^{1}\right)\right)$ is equal to the Cartesian product space $\left(\times_{i=1}^{n} \mathscr{T}_{k}\left(C_{i}\right)\right) \times\left(\times_{i=1}^{n} \mathscr{T}_{k}\left(S^{1}\right)\right)$. Thus, we may define a map

$$
J_{n}: \mathscr{T}_{k}(M) \rightarrow \mathscr{T}_{k}\left(\left(\bigcup_{i=1}^{n} C_{i}\right) \cup\left(\bigcup_{i=1}^{n} S^{1}\right)\right)
$$

by taking the products of all the maps $r_{i}: \mathscr{T}_{k}(M) \rightarrow \mathscr{T}_{k}\left(C_{i}\right), i=1, \ldots, n$, with all the maps $s_{i}: \mathscr{T}_{k}(M) \rightarrow \mathscr{T}_{k}\left(S^{1}\right), i=1, \ldots, n$. The map $J_{n}$ is a left homotopy inverse to the map $J^{n}$ because the composite $J_{n} \circ J^{n}$ can be seen to induce an isomorphism of all homotopy groups. The reader is referred to [25, $\S 2]$ for more details.

This completes Step I in the proof of Theorem A.1.

Step II. In this step, we complete the proof of Theorem A.1 by showing that the map $J: \mathscr{T}_{k}\left(\left(\bigcup_{i} C_{i}\right) \cup\left(\bigcup_{i} S^{1}\right)\right) \rightarrow \mathscr{T}_{k}(M)$ induces a surjection on all the homotopy groups. To do this, it will suffice to show that for any continuous map $f: K \rightarrow \mathscr{T}_{k}(M)$ from a finite $C W$ complex $K$ there is a homotopy $f_{t}: K \rightarrow \mathscr{T}_{k}(M), t \in[0,3]$, and a map $g: K \rightarrow \mathscr{T}_{k}\left(\left(\cup_{i} C_{i}\right) \cup\left(\bigcup_{i} S^{1}\right)\right)$ that satisfy the following.

A.9. $f_{3}=J \circ g$.

The construction of the homotopy $f_{t}, t \in[0,3]$, and the map $g$ of A.9 proceeds as in $[25, \S \S 5,6]$, so we will be brief. We may assume without loss of generality that $M$ equals the topological product $M^{\prime} \times R$ equipped with the Riemann metric of (3.8.4), where $M^{\prime}$ is a complete hyperbolic manifold. Thus, we have the asymptotic northern hemisphere subbundle $D(T(M))$ of the tangent bundle for $M$, and we have the asymptotic foliation $\mathscr{F}_{D}$ for $D(T(M))$ and the foliation $\mathscr{G}$ of $D(T(M))$ by the trajectories of the geodesic flow 
$G^{t}: D(T(M)) \rightarrow D(T(M)), t \in R$. First we use the asymptotic transfer construction of $[25, \S 5]$ to lift $f$ to a map $\hat{f}: K \rightarrow \mathscr{T}_{k}(D(T(M)))$. The map $\hat{f}$ is $(\alpha, 0)$-controlled over $\left(D(T(M)), \mathscr{F}_{c}\right)$ (for some positive number $\left.\alpha\right)$. Next we vary $\hat{f}$ through a homotopy $\hat{f}_{t}: K \rightarrow \mathscr{T}_{k}(D(T(M))), t \in[0,1]$, as follows.

A.10. Let $\gamma$ denote a fixed but very large positive number. For each stable pseudo-isotopy $h:\left(D(T(M)) \times R^{k} \times I^{u}\right) \times I \rightarrow\left(D(T(M)) \times R^{k} \times I^{u}\right) \times I$ in $\mathscr{T}_{k}(D(T(M)))$, which represents $f(x)$ for some $x \in K$, set

$$
\hat{f}_{t}(x)=\left(G^{t \gamma} \times \mathrm{id}\right) \circ h \circ\left(G^{-t \gamma} \times \mathrm{id}\right),
$$

where id: $R^{k} \times I^{u} \times I \rightarrow R^{k} \times I^{u} \times I$ is the identity map.

Note that the map $\hat{f}_{1}: K \rightarrow \mathscr{T}_{k}(D(T(M)))$ is $(\alpha, \delta)$-controlled over $(D(T(M)), \mathscr{G})$, where $\delta$ is a positive number satisfying $\lim _{\gamma \rightarrow \infty} \delta=0$. For any positive number $\alpha^{\prime}$, let $\left\{L_{i}\right\}$ denote the collection of all closed flow lines for the geodesic flow $G^{t}: D(T(M)) \rightarrow D(T(M)), t \in R$, which have length less than or equal to $\alpha^{\prime}$. Choose very small pairwise disjoint tubular neighborhoods $\left\{T_{i}\right\}$ for the $\left\{L_{i}\right\}$ in $D(T(M))$. For any positive numbers $\varepsilon, \beta$, the subset $D_{\varepsilon, \beta}(T(M)) \subset D(T(M))$ has been defined in 8.1. Note that $\mathscr{G}$ is uniformly $\left(\alpha^{\prime} / 2,0\right)$-flat on the subset $D_{\varepsilon, \beta}(T(M))-\left(\bigcup_{i} T_{i}\right)$ (see Addendum 3.5.5). So if $\alpha^{\prime}$ is choosen sufficiently larger than $\alpha$, then we may use the foliated control theorem [28, A.3] to get a homotopy $\hat{f}_{t}: K \rightarrow \mathscr{T}_{k}(D(T(M))), t \in[1,2]$, of $\hat{f}_{1}$ that satisfies the following property.

A.11. (a) For each $x \in K$, the support of the pseudo-isotopy $\hat{f}_{2}(x)$ lies over the subset $\left(D(T(M))-D_{\varepsilon, \beta}(T(M))\right) \cup\left(\bigcup_{i=1}^{n} T_{i}\right)$, for some sufficiently large positive integer $n$ ( $n$ is independent of $x$ ).

(b) Under the projection $D(T(M)) \rightarrow M$, we have that $\hat{f}_{2}$ is $\alpha^{\prime}$-controlled over all of $M$.

Recall that $M$ is the topological product $M^{\prime} \times R$ equipped with the metric of (3.8.4), where $M^{\prime}$ is a complete hyperbolic manifold. It follows from A.11 that $\hat{f}_{2}$ is $\delta^{\prime}$-controlled over $M^{\prime^{\varepsilon}} \times R-\bigcup_{i=1}^{n} T_{i}^{\prime}$ with respect to the product metric on $M^{\prime} \times R$, where $\delta^{\prime}$ is a positive number satisfying $\lim _{\beta \rightarrow \infty} \delta^{\prime}=0$ and where $\left\{T_{i}^{\prime}\right\}$ are tubular neighborhoods of embedded circles in $M^{\prime} \times R$. Note that the composite map $D(T(M)) \stackrel{\text { proj }}{\rightarrow} M \stackrel{\cong}{\rightrightarrows} M^{\prime} \times R$ induces an equivalence $\mathscr{T}(D(T(M))) \cong \mathscr{T}\left(M^{\prime} \times R\right)$. Under this equivalence, the homotopy $\hat{f}_{t}, t \in[0,2]$, of A.10 and A.11 becomes identified with a homotopy $f_{t}: K \rightarrow$ $\mathscr{T}_{k}\left(M^{\prime} \times R\right), t \in[0,2]$, of the map $f: K \rightarrow \mathscr{T}_{k}(M)$, such that $f_{2}$ is $\delta^{\prime}$ controlled over $M^{\prime^{\varepsilon}} \times R-\bigcup_{i=1}^{n} T_{i}^{\prime}$. Since the foliation of $M^{\prime} \times R$ by its points is uniformly $\left(\varepsilon^{\prime}, \sigma\right)$-flat on the subset $M^{\prime^{\varepsilon}} \times R$, for $\varepsilon^{\prime}$ sufficiently small and for $\sigma$ as in [28, A.3], we may apply [28, A.3] to $f_{2}$ to get a homotopy $f_{t}: \rightarrow \mathscr{T}_{k}\left(M^{\prime} \times R\right), t \in[2,3]$, of $f_{2}$ such that the following is true.

(c) For each $x \in K$, the support of the pseudo-isotopy $f_{3}(x)$ lies over the subset $\left(\left(M^{\prime}-M^{\prime^{i}}\right) \times R\right) \cup\left(\bigcup_{i=1}^{n} T_{i}^{\prime}\right)$. 
Note that for sufficiently small $\varepsilon$ in A.11(c), it will follow from A.11(c) that there is a map $g: K \rightarrow \mathscr{T}_{k}\left(\left(\cup_{i} C_{i}\right) \cup\left(\bigcup_{i} S^{1}\right)\right)$ such that $f_{3}$ and $g$ satisfy A.9.

This completes the proof of Theorem A.1.

Proof of Theorem A.2. We will need the following notation. Let $X$ denote a connected smooth manifold, $Y$ an open subset of $X, D$ a compact subset of $X$, and $\varepsilon$ a positive number. We denote by $\mathscr{D}(X ; Y, \varepsilon, D)$ the set of all stable smooth pseudo-isotopies in $\mathscr{D}(X)$ that are $\varepsilon$-controlled over $Y$ and whose support lies over the subset $D$. Denote by $C \mathscr{D}(X ; Y, D)$ the space of all paths $p:[0, \infty) \rightarrow \mathscr{D}(X)$ such that $p(t) \in \mathscr{D}\left(X ; Y,(t+1)^{-1}, D\right)$ holds for all $t \in$ $[0, \infty)$. Define $C \mathscr{D}(X ; Y)$ to be the direct limit space $\lim _{D \rightarrow X} C \mathscr{D}(X ; Y, D)$.

By applying the arguments given in [65] (see, in particular, $\S 6$ and the appendix) to the function $\mathscr{D}($ ) (instead of to $\mathscr{T}()$ ), we can derive the following.

A.12. (a) $C \mathscr{D}(X)$ is an $\Omega$-spectrum. (Here $C \mathscr{D}(X)=C \mathscr{D}(X ; X)$.)

(b) There is an equivalence of $\Omega$-spectra $C \mathscr{D}(X) \cong \mathbf{H}(X, \mathscr{D}(*))$.

Now A.12(b) allows us to define the amalgamation map $A: \mathbf{H}(X ; \mathscr{D}(*)) \rightarrow$ $\mathscr{D}(X)$ as the map that sends $p \in C \mathscr{D}(X)$ to the stable pseudo-isotopy $p(0)$.

The remainder of the proof of Theorem A.2 is divided into the following four steps.

Step I. In this step, we verify the following.

A.13. Let $N$ denote any compact closed flat Riemannian manifold. Then there is an equivalence of $\Omega$-spectra $\mathscr{D}(N) \cong C \mathscr{D}(N) \times N \mathscr{D}(N)$.

In verifying A.13, the only geometric property of $N$ that we use is that there are positive integers $p, q$ and maps $i_{p}: N \rightarrow N, i_{q}: N \rightarrow N$ that satisfy the following properties (see [12]).

A.14. (a) $i_{p}$ and $i_{q}$ are expanding self-immersions of $N$.

(b) $p$ and $q$ are relatively prime integers, and $i_{p}, i_{q}$ have homological degrees of $p, q$, respectively.

(c) $i_{p} \circ i_{q}=i_{q} \circ i_{p}$. Set $i_{p q}=i_{q} \circ i_{p}$.

For $x=p, q$, or $p q$, we may pull pseudo-isotopies in $\mathscr{D}(N)$ back along the immersion $i_{x}: N \rightarrow N$. This pullback construction is denoted by $T_{x}: \mathscr{D}(N) \rightarrow$ $\mathscr{D}(N)$. Note that $T_{x}: \mathscr{D}(N) \rightarrow \mathscr{D}(N)$ is a mapping of $\Omega$-spectra. The same pullback construction may be applied to the $C \mathscr{D}(N)$ to get a mapping $C T_{X}: C \mathscr{D}(N) \rightarrow C \mathscr{D}(N)$ of $\Omega$-spectra. Let $C \mathscr{D}^{x}(N)$ and $\mathscr{D}^{x}(N)$ denote the direct limits of the sequence

$$
C \mathscr{D}(N) \stackrel{C T_{x}}{\rightarrow} C \mathscr{D}(N) \stackrel{C T_{x}}{\rightarrow} C \mathscr{D}(N) \stackrel{C T_{x}}{\rightarrow} \cdots
$$

and

$$
\mathscr{D}(N) \stackrel{T_{x}}{\rightarrow} \mathscr{D}(N) \stackrel{T_{x}}{\rightarrow} \mathscr{D}(N) \stackrel{C T_{x}}{\rightarrow} \ldots .
$$

Note that the map $A: C \mathscr{D}(N) \rightarrow \mathscr{D}(N)$ induces a map $A^{x}: C \mathscr{D}^{x}(N) \rightarrow$ $\mathscr{D}^{x}(N)$ of $\Omega$-spectra. Note that the maps $T_{p}, C T_{p}$ induce maps $J_{p}: \mathscr{D}^{q}(N)$ $\rightarrow \mathscr{D}^{p q}(N)$ and $C J_{p}: C \mathscr{D}^{q}(N) \rightarrow C \mathscr{D}^{p q}(N)$, and the maps $T_{q}, C T_{q}$ induce 
maps $J_{q}: \mathscr{D}^{p}(N) \rightarrow \mathscr{D}^{p q}(N)$ and $C J_{q}: C \mathscr{D}^{p}(N) \rightarrow C \mathscr{D}^{p q}(N)$. (For example, A.14(c) guarantees that each square in the sequence

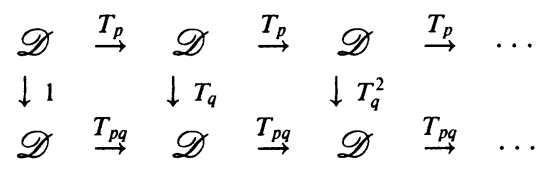

is commutative. Thus, the collection of all vertical maps gives $J_{q}$.)

For $x=p, q, p q$, let $C \mathscr{D}^{x, x}(N)$ and $\mathscr{D}^{x, x}(N)$ denote the localizations of $C \mathscr{D}^{x}(N)$ and $\mathscr{D}^{x}(N)$ at the set of primes $\bar{x}$, where $\bar{x}$ denotes all the primes except those prime factors of $x$. Note that $A^{x}$ induces a map $A^{x, x}: C \mathscr{D}^{x, x}(N)$ $\rightarrow \mathscr{D}^{x, x}(N)$ of $\Omega$-spectra, and the maps $C J_{x}, J_{x}$ (for $\left.x=p, q\right)$ induce maps of $\Omega$-spectra

$$
\begin{array}{ll}
C J_{q, q}: C \mathscr{D}^{p, p}(N) \rightarrow C \mathscr{D}^{p q, p q}(N), & J_{q, q}: \mathscr{D}^{p, p}(N) \rightarrow \mathscr{D}^{p q, p q}(N), \\
C J_{p, p}: C \mathscr{D}^{q, q}(N) \rightarrow C \mathscr{D}^{p q, p q}(N), & J_{p, p}: \mathscr{D}^{q, q}(N) \rightarrow \mathscr{D}^{p q, p q}(N) .
\end{array}
$$

We claim that all of the maps $A^{x, x}, J_{x, x}$, and $C J_{x, x}$ satisfy the following properties.

A.15. (a) For $x=p, q$, or $p q$, the map $A^{x, x}: C \mathscr{D}^{x, x}(N) \rightarrow \mathscr{D}^{x, x}(N)$ is an equivalence of $\Omega$-spectra.

(b) The $\Omega$-spectrum $C \mathscr{D}(N)$ is the fiber product of the diagram

$$
\begin{array}{cc} 
& C \mathscr{D}^{q, q}(N) \\
& \downarrow J_{p, p} \\
C \mathscr{D}^{p, p}(N) \stackrel{J_{q, q}}{\rightarrow} & C \mathscr{D}^{p q, p q}(N)
\end{array}
$$

In verifying A.15(b), it will suffice to show that $C \mathscr{D}(N) \rightarrow C \mathscr{D}^{p, p}(N)$ and $C \mathscr{D}(N) \rightarrow C \mathscr{D}^{q, q}(N)$ are the localizations of $C \mathscr{D}(N)$ at the sets of primes $\bar{p}$ and $\bar{q}$, and that both

$$
C \mathscr{D}(N) \rightarrow C \mathscr{D}^{p, p}(N) \stackrel{J_{q, q}}{\rightarrow} C \mathscr{D}^{p q, p q}(N)
$$

and

$$
C \mathscr{D}(N) \rightarrow C \mathscr{D}^{q, q}(N) \stackrel{J_{p} p}{\rightarrow} C \mathscr{D}^{p q, p q}(N)
$$

are the localization of $C \mathscr{D}(N)$ at the set of primes $\overline{p q}$. To see this, it is enough to check that $C T_{x}: C \mathscr{D}(N) \rightarrow C \mathscr{D}(N)$ is an equivalence modulo $x$-torsion. Let $C I_{x}: C \mathscr{D}(N) \rightarrow C \mathscr{D}(N)$ denote the map induced by $i_{x}: N \rightarrow N$. Note that in terms of the equivalence of A.12(b) the map $C I_{x} \circ C T_{x}: \mathbf{H}(N ; \mathscr{D}(*)) \rightarrow$ $\mathbf{H}(N ; \mathscr{D}(*))$ is induced by the identity map id: $N \rightarrow N$ and by multiplication by $x$ in the $\Omega$-spectra $\mathscr{D}(*)$. Thus, $C I_{x} \circ C T_{x}: C \mathscr{D}(N) \rightarrow C \mathscr{D}(N)$ is an equivalence modulo $x$-torsion of $\Omega$-spectra. Note also that in terms of the equivalence of A.12(b) the map $C I_{x}: \mathbf{H}(N ; \mathscr{D}(*)) \rightarrow \mathbf{H}(N ; \mathscr{D}(*))$ is induced by the identity map id: $\mathscr{D}(*) \rightarrow \mathscr{D}(*)$ in the $\Omega$-spectrum of coefficients $\mathscr{D}(*)$ and by the equivalence modulo $x$-torsion $i_{x}: N \rightarrow N$. Thus, $C I_{x}: C \mathscr{D}(N) \rightarrow$ $C \mathscr{D}(N)$ is also an equivalence modulo $x$-torsion of $\Omega$-spectra. It follows now 
that $C T_{x}: C \mathscr{D}(N) \rightarrow C \mathscr{D}(N)$ must be an equivalence modulo $x$-torsion of $\Omega$-spectra.

To verify A.15(a), we use the fact that $C T_{x}: C \mathscr{D}(N) \rightarrow C \mathscr{D}(N)$ is an equivalence modulo $x$-torsion of $\Omega$-spectra. We shall also use the fact that, for each pseudo-isotopy $h \in \mathscr{D}(N)$, we have that, for large enough $n$, the pseudoisotopy $T_{x}^{n}(h)$ is sufficiently well controlled over $N$ that the arguments of $\S 6$ in [65] apply (in the smooth category) to show that $T_{x}^{n}(h) \in \operatorname{image}(A)$. The remaining details in the verification of A.15(a) are left to the reader.

Let $r_{x, x}: \mathscr{D}(N) \rightarrow C \mathscr{D}^{x, x}(N)$ denote the composite map $\mathscr{D}(N) \rightarrow \mathscr{D}^{x, x}(N)$ $\cong C \mathscr{D}^{x, x}(N)$ (see A.15(a)). Note that in the following diagram all the solid arrows commute up to homotopy.

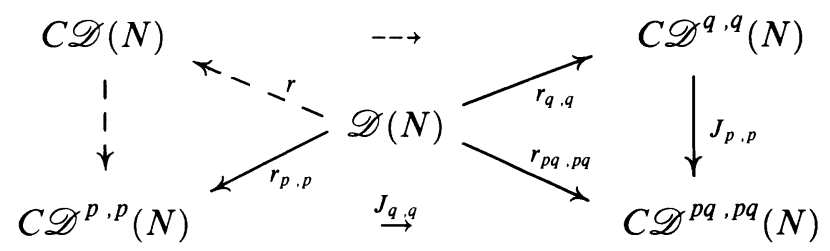

Thus, the map $r: \mathscr{D}(N) \rightarrow C \mathscr{D}(N)$ may be defined to be the fiber product of $r_{p, p}$ and $r_{q, q}$. We leave it to the reader to check that $r: \mathscr{D}(N) \rightarrow C \mathscr{D}(N)$ is a left homotopy inverse to the map $A: C \mathscr{D}(N) \rightarrow \mathscr{D}(N)$ in the category of $\Omega$-spectra. It follows that $\mathscr{D}(N)$ splits as a product of $C \mathscr{D}(N)$ and the kernel of $r$, which verifies A.13.

This completes Step I.

Step II. Approximate each of the immersed closed geodesics $g_{i}: S^{1} \rightarrow M$ by pairwise disjoint smooth embeddings $\bar{g}_{i}: S^{1} \rightarrow M$. Choose closed tubular neighborhoods $\left\{T_{i}\right\}$ for the $\left\{\bar{g}_{i}\left(S^{1}\right)\right\}$ in $M$, and choose closed tubular neighborhoods $\left\{U_{i}\right\}$ for the cusps $\left\{\mathscr{C}_{i}\right\}$ of $M$ in $M$, all of which satisfy the following: $T_{i} \cap T_{j}=\varnothing(i \neq j) ; U_{i} \cap U_{j}=\varnothing(i \neq j) ; T_{i} \cap U_{j}=\varnothing$. In the remainder of this step, $n$ will denote a fixed but arbitrary positive integer and

$$
Y=M-\left[\left(\bigcup_{i=1}^{n} U_{i}\right) \cup\left(\bigcup_{i=1}^{n} T_{i}\right)\right] .
$$

The purpose of this step is to verify the following property.

A.16. There is an equivalence of $\Omega$-spectra

$$
C \mathscr{D}(M ; Y) \cong C \mathscr{D}(M) \times N \mathscr{D}\left(\left(\bigcup_{i=1}^{n} C_{i}\right) \cup\left(\bigcup_{i=1}^{n} S^{1}\right)\right) .
$$

We first describe our candidate for an equivalence

$$
f: C \mathscr{D}(M) \times N \mathscr{D}\left(\left(\bigcup_{i=1}^{n} C_{i}\right) \cup\left(\bigcup_{i=1}^{n} S^{1}\right)\right) \rightarrow C \mathscr{D}(M ; Y)
$$


of $\Omega$-spectra. Let

$$
f_{1}: C \mathscr{D}(M) \times N \mathscr{D}\left(\left(\bigcup_{i=1}^{n} C_{i}\right) \cup\left(\bigcup_{i=1}^{n} S^{1}\right)\right) \rightarrow C \mathscr{D}(M ; Y)
$$

denote the composite map

$$
C \mathscr{D}(M) \times N \mathscr{D}\left(\left(\bigcup_{i=1}^{n} C_{i}\right) \cup\left(\bigcup_{i=1}^{n} S^{1}\right)\right) \stackrel{\text { proj }}{\rightarrow} C \mathscr{D}(M) \stackrel{\text { inclusion }}{\rightarrow} C \mathscr{D}(M ; Y) .
$$

Let

$$
f_{2}: C \mathscr{D}(M) \times N \mathscr{D}\left(\left(\bigcup_{i=1}^{n} C_{i}\right) \cup\left(\bigcup_{i=1}^{n} S^{1}\right)\right) \rightarrow C \mathscr{D}(M ; Y)
$$

denote the composite map

$$
\begin{array}{r}
C \mathscr{D}(M) \times N \mathscr{D}\left(\left(\bigcup_{i=1}^{n} C_{i}\right) \cup\left(\bigcup_{i=1}^{n} S^{1}\right)\right) \stackrel{\text { proj }}{\rightarrow} N \mathscr{D}\left(\left(\bigcup_{i=1}^{n} C_{i}\right) \cup\left(\bigcup_{i=1}^{n} S^{1}\right)\right) \\
\stackrel{\text { inclusion }}{\longrightarrow} \mathscr{D}\left(\left(\bigcup_{i=1}^{n} C_{i}\right) \cup\left(\bigcup_{i=1}^{n} S^{1}\right)\right) \stackrel{I}{*}^{*} C \mathscr{D}(M ; Y),
\end{array}
$$

where the inclusion map exists by virtue of A.13 and where $I^{*}$ is the map induced by $I \mid\left(\left(\bigcup_{i=1}^{n} C_{i}\right) \cup\left(\bigcup_{i=1}^{n} S^{1}\right)\right.$ ) (see Theorem A.1 for $\left.I\right)$. Now set $f=$ $f_{1}+f_{2}$, where addition takes place in the $\Omega$-spectrum $C \mathscr{D}(M ; Y)$.

Note that both the restriction maps $r_{1}: C \mathscr{D}_{k}(M) \rightarrow C \mathscr{D}_{k}(Y)$ and $r_{2}$ : $C \mathscr{D}_{k}(M ; Y) \rightarrow C \mathscr{D}_{k}(Y)$ are well-defined homotopy fibrations. (To see this, use the arguments of the appendix in [65] for the smooth category.) Thus, the composite map

$$
C \mathscr{D}_{k}(M) \times N \mathscr{D}_{k}\left(\left(\bigcup_{i=1}^{n} C_{i}\right) \cup\left(\bigcup_{i=1}^{n} S^{1}\right)\right) \stackrel{\text { proj }}{\rightarrow} C \mathscr{D}_{k}(M) \stackrel{r_{1}}{\rightarrow} C \mathscr{D}_{k}(Y)
$$

is also a homotopy fibration. Note that $f$ is a map of homotopy fibrations that covers the identity map $C \mathscr{D}_{k}(Y) \rightarrow C \mathscr{D}_{k}(Y)$ (i.e., $\left.r_{2} \circ f=\bar{r}_{1}\right)$. Note also that by A.13 $f$ maps the fiber $\bar{r}_{1}^{-1}(p)$ homotopy equivalently to the fiber $r_{2}^{-1}(p)$, where $p$ is the point of $C \mathscr{D}_{k}(Y)$ represented by the identity pseudoisotopy. Thus, $f$ induces isomorphisms between the homotopy groups of the base spaces of $\bar{r}_{1}$ and $r_{2}$ and between the homotopy groups of the fibers of $\bar{r}_{1}$ and $r_{2}$. It follows that $f$ is a homotopy equivalence of the total space of $\bar{r}_{1}$ to the total space of $r_{2}$. Thus, $f$ is the desired equivalence of A.16.

This completes Step II in the proof of Theorem A.2.

Step III. In this step, we verify Theorem A.2(c).

Let $X$ denote any smooth manifold, let $\mathscr{T} / \mathscr{D}(X)$ denote the $\Omega$-spectrum that is the cofiber of the forgetful map $\mathscr{D}(X) \rightarrow \mathscr{T}(X)$, and let $\mathscr{D}^{1}(X)$ de- 
note the $\Omega$-spectrum that is the one-fold delooping of $\mathscr{D}(X)$ (i.e., $\mathscr{D}_{k}^{1}(X)=$ $\left.\mathscr{D}_{k+1}(X)\right)$. Note that there is a fibration $\mathscr{T}(X) \rightarrow \mathscr{T} / \mathscr{D}(X) \rightarrow \mathscr{D}^{1}(X)$ in the category of $\Omega$-spectra.

Let $\mathscr{G}($ ) denote any functor from smooth manifolds to $\Omega$-spectra. Then we can define the homology spectrum $\mathbf{H}(X ; \mathscr{G}(*))$ and the amalgamation map $A: \mathbf{H}(X ; \mathscr{G}(*)) \rightarrow \mathscr{G}(X)$ by simply substituting $\mathscr{G}(*), \mathscr{G}(X)$ for $\mathscr{D}(*), \mathscr{D}(X)$ in the definition of $A: \mathbf{H}(X ; \mathscr{D}(*)) \rightarrow \mathscr{D}(X)$ given above. Let $N \mathscr{G}(X)$ denote the $\Omega$-spectrum that is the cofiber of the amalgamation map $A: \mathbf{H}(X ; \mathscr{G}(*)) \rightarrow$ $\mathscr{G}(X)$, and let $\mathscr{G}^{\prime}(X)$ denote the $\Omega$-spectrum that is the one-fold delooping of $\mathscr{G}(X)$. Note that there is a fibration in the category of $\Omega$-spectra $N \mathscr{G}(X) \rightarrow$ $\mathbf{H}\left(X ; \mathscr{G}^{1}(*)\right) \rightarrow \mathscr{G}^{1}(X)$.

We consider the following homotopy commutative diagram of maps:

$$
\begin{aligned}
& \mathbf{H}_{0}(X ; \mathscr{T} / \mathscr{D}(*)) \stackrel{r_{1}}{\rightarrow} \quad \mathbf{H}_{0}\left(X ; \mathscr{D}^{1}(*)\right) \\
& \begin{array}{ccc}
\downarrow r_{2} & & \downarrow r_{4} \\
\mathscr{T} / \mathscr{D}_{0}(X) & \overrightarrow{r_{3}} & \mathscr{D}_{0}^{1}(X)
\end{array}
\end{aligned}
$$

Note that $\mathscr{T} / \mathscr{D}(*) \cong \mathscr{D}^{1}(*)$, so $r_{1}$ is a homotopy equivalence. The functor $\mathscr{T} / \mathscr{D}_{0}()$ is a homology functor (cf. $[7,8,10]$ ), so $r_{2}$ is a homotopy equivalence. On the other hand, the fiber of $r_{3}$ is equal to $\mathscr{P}(X)$ and the fiber of $r_{4}$ is equal to $N \mathscr{D}_{0}(X)$. Thus, the homotopy equivalence $r_{2} \circ r_{1}^{-1}: \mathbf{H}_{0}\left(X ; \mathscr{D}^{1}(*)\right) \rightarrow$ $\mathscr{T} / \mathscr{D}_{0}(X)$ gives rise to a homotopy equivalence $N \mathscr{D}_{0}(X) \cong \mathscr{P}(X)$. Now letting $X=\left(\bigcup_{i} C_{i}\right) \cup\left(\bigcup_{i} S^{1}\right)$ completes the proof of Theorem A.2(c).

This completes Step III.

Step IV. In this step, we complete the verification of both Theorem A.2(a) and (b). We do this by showing that a certain map

$$
F: C \mathscr{D}_{0}(M) \times N \mathscr{D}_{0}\left(\left(\bigcup_{i} C_{i}\right) \cup\left(\bigcup_{i} S^{1}\right)\right) \rightarrow \mathscr{D}_{0}(M)
$$

is a homotopy equivalence.

We shall first describe the map $F$. Let

$$
F_{1}: C \mathscr{D}_{0}(M) \times N \mathscr{D}_{0}\left(\left(\bigcup_{i} C_{i}\right) \cup\left(\bigcup_{i} S^{1}\right)\right) \rightarrow \mathscr{D}_{0}(M)
$$

denote the composite map

$$
C \mathscr{D}_{0}(M) \times N \mathscr{D}_{0}\left(\left(\bigcup_{i} C_{i}\right) \cup\left(\bigcup_{i} S^{1}\right)\right) \stackrel{\text { proj }}{\rightarrow} C \mathscr{D}_{0}(M) \stackrel{\text { inclusion }}{\rightarrow} \mathscr{D}_{0}(M) .
$$

Let

$$
F_{2}: C \mathscr{D}_{0}(M) \times N \mathscr{D}_{0}\left(\left(\bigcup_{i} C_{i}\right) \cup\left(\bigcup_{i} S^{1}\right)\right) \rightarrow \mathscr{D}_{0}(M)
$$


denote the composite map

$$
\begin{array}{r}
C \mathscr{D}_{0}(M) \times N \mathscr{D}_{0}\left(\left(\bigcup_{i} C_{i}\right) \cup\left(\bigcup_{i} S^{1}\right)\right) \stackrel{\text { proj }}{\rightarrow} N \mathscr{D}_{0}\left(\left(\bigcup_{i} C_{i}\right) \cup\left(\bigcup_{i} S^{1}\right)\right) \\
\stackrel{\text { inclusion }}{\rightarrow} \mathscr{D}_{0}\left(\left(\bigcup_{i} C_{i}\right) \cup\left(\bigcup_{i} S^{1}\right)\right) \stackrel{I^{*}}{\rightarrow} \mathscr{D}_{0}(M),
\end{array}
$$

where the inclusion map exists by virtue of A.13 and where $I^{*}$ is the map induced by $I$. Now set $F=F_{1}+F_{2}$, where the addition takes place in the $\Omega$-spectrum $\mathscr{D}(M)$.

We first show that $F$ induces a surjection on the homotopy groups. Let $\alpha \in \pi_{l}\left(\mathscr{D}_{0}(M)\right)$ be an arbitrary homotopy group element (with $l$ also arbitrary), and let $K \rightarrow \mathscr{D}_{0}(M)$ be a map from the $l$-dimensional sphere $K$ that represents $\alpha$. If we apply the smooth version of the argument of Step II in the proof of Theorem A.1 to the map $K \rightarrow \mathscr{D}_{0}(M)$, we conclude that $\alpha$ is in the image of the inclusion induced map

$$
\pi_{l}\left(C \mathscr{D}_{0}(M ; Y)\right) \rightarrow \pi_{l}\left(\mathscr{D}_{0}(M)\right),
$$

where $Y \subset M$ is one of the subsets in A.16. But the equivalence of A.16 (i.e., the map $f$ constructed in Step II of this proof) when composed with the inclusion $C \mathscr{D}_{0}(M ; Y) \rightarrow \mathscr{D}_{0}(M)$ is equal to the restricted map $F \mid C \mathscr{D}_{0}(M) \times$ $N \mathscr{D}_{0}\left(\left(\bigcup_{i=1}^{n} C_{i}\right) \cup\left(\bigcup_{i=1}^{n} S^{1}\right)\right)$. It follows now that $\alpha$ is in the image of the $F$ induced map

$$
\pi_{l}\left(C \mathscr{D}_{0}(M) \times N \mathscr{D}_{0}\left(\left(\bigcup_{i} C_{i}\right) \cup\left(\bigcup_{i} S^{1}\right)\right) \stackrel{F_{l}}{\rightarrow} \pi_{l}\left(\mathscr{D}_{0}(M)\right) .\right.
$$

(Note that to apply the smooth version of the argument in Step II of the proof of Theorem A.1, we must appeal to the smooth foliated control theorem in [28] instead of appealing to the topological foliated control theorem of [28].)

Now we will show that $F$ induces an injection of the homotopy groups. Choose an $\alpha \in \operatorname{kernel}\left(F_{l}\right)$. Because the composite map

$$
\begin{gathered}
N \mathscr{D}_{0}\left(\left(\bigcup_{i} C_{i}\right) \cup\left(\bigcup_{i} S^{1}\right)\right) \stackrel{\text { inclusion }}{\rightarrow} C \mathscr{D}_{0}(M) \times N \mathscr{D}_{0}\left(\left(\bigcup_{i} C_{i}\right) \cup\left(\bigcup_{i} S^{1}\right)\right) \\
\stackrel{F}{\rightarrow} \mathscr{D}_{0}(M) \stackrel{\text { forgetful map }}{\rightarrow} \mathscr{T}_{0}(M)
\end{gathered}
$$

is a homotopy equivalence (by Theorem A.1 and Step III of this proof), it follows that $\alpha \in \operatorname{kernel}\left(A_{l}\right)$, where $A_{l}: \pi_{l}\left(C \mathscr{D}_{0}(M)\right) \rightarrow \pi_{l}\left(\mathscr{D}_{0}(M)\right)$ is the map induced by $A: C \mathscr{D}_{0}(M) \rightarrow \mathscr{D}_{0}(M)$. Represent $\alpha$ by a map $g: S^{l} \rightarrow C \mathscr{D}_{0}(M)$, and let $G: D^{l+1} \rightarrow \mathscr{D}_{0}(M)$ be a null homotopy for $G \mid \partial D^{l+1}=F \circ g$. We apply the smooth version of the argument of Step II in the proof of Theorem A.1 to the map $G: D^{l+1} \rightarrow \mathscr{D}_{0}(M)$ to get homotopies $g_{t}: S^{l} \rightarrow C \mathscr{D}_{0}(M), t \in[0,1]$, and $G_{t}: D^{l+1} \rightarrow \mathscr{D}_{0}(M), t \in[0,1]$, that satisfy the following properties. 
A.17. (a) $F \circ g_{t}=G_{t} \mid \partial D^{l+1}$ for all $t \in[0,1]$.

(b) Image $\left(G_{1}\right) \subset C \mathscr{D}_{0}(M ; Y)$ for some $Y$ as in A.16.

Since the restricted map $F \mid\left[C \mathscr{D}_{0}(M) \times N \mathscr{D}_{0}\left(\left(\bigcup_{i=1}^{n} C_{i}\right) \cup\left(\bigcup_{i=1}^{n} S^{1}\right)\right)\right]$ is the equivalence of A.16, it follows from A.17 that the map $g_{1}: S^{l} \rightarrow C \mathscr{D}_{0}(M)$ is null homotopic in $C \mathscr{D}_{0}(M)$. Thus, $\alpha=0$, showing that $F_{l}$ is an injective map.

This completes the proof of Theorem A.2.

Remark A.18. In this remark, we formulate fiber bundle versions of Theorems A.1 and A.2. We note that the proofs of these more general theorems follow the same pattern as the proof of Theorem A.2.

Let $p: E \rightarrow M$ denote a homotopy fibration over the smooth manifold $M$ (with $\partial M=\varnothing$ ). Following the ideas of [65, Appendix], we define the "homology $\Omega$-spectrum" for $\mathscr{T}($ ) over $M$, denoted by $\mathbf{H}(M ; \mathscr{T}(p))$, as follows. Choose a triangulation $K$ for $M$. Define $\mathscr{T}_{j}(p)$ to be

$$
\left(\bigcup_{\Delta \in K} \mathscr{T}_{j}\left(p^{-1}(\Delta)\right) \times \Delta\right) / \approx,
$$

where the equivalence relation identifies $\mathscr{T}_{j}\left(p^{-1}(\Delta)\right) \times \Delta$ with $\mathscr{T}_{j}\left(p^{-1}\left(\Delta^{\prime}\right)\right) \times \Delta^{\prime}$ via the inclusion induced map for every pair $\Delta, \Delta^{\prime} \in K$ with $\Delta \subset \Delta^{\prime}$. There is a natural inclusion map $i: M \rightarrow \mathscr{T}_{j}(M)$ defined by fitting together the base points of the pieces $\mathscr{T}_{j}\left(p^{-1}(\Delta)\right)$. The delooping maps $\mathscr{T}_{j}() \rightarrow \Omega \mathscr{T}_{j+1}()$ induce maps $\mathscr{T}_{j}(p) / i(M) \rightarrow \Omega\left(\mathscr{T}_{j+1}(p) / i(M)\right)$. We define the $j$ th element in the $\Omega$-spectrum $\mathbf{H}(M ; \mathscr{T}(p))$ by

$$
\mathbf{H}_{j}(M ; \mathscr{T}(p))=\lim _{i \rightarrow \infty} \Omega^{i}\left(\mathscr{T}_{j+i}(p) / i(M)\right) .
$$

There is a natural map of $\Omega$-spectra $A: \mathbf{H}(M ; \mathscr{T}(p)) \rightarrow \mathscr{T}(E)$ called the amalgamation map. At the $j$ th level, $A$ is defined to be the composite

$$
\lim _{i \rightarrow \infty} \Omega^{i}\left(\mathscr{T}_{j+i}(p) / i(M)\right) \stackrel{\psi}{\rightarrow} \lim _{i \rightarrow \infty} \Omega^{i} \mathscr{T}_{j+i}(E) \cong \mathscr{T}_{j}(E),
$$

where $\psi$ is induced by the union of the inclusion induced maps $\mathscr{T}_{j+i}\left(p^{-1}(\Delta)\right)$ $\rightarrow \mathscr{T}_{j+i}(E)$. We denote by $N \mathscr{T}(p)$ the cokernel $\Omega$-spectrum for the amalgamation map $A: \mathbf{H}(M ; \mathscr{T}(p)) \rightarrow \mathscr{T}(E)$.

If in the preceding paragraph we replace the $\Omega$-spectrum valued functor $\mathscr{T}()$ by the $\Omega$-spectrum valued functor $\mathscr{D}()$, then we obtain the homology $\Omega$ spectrum $\mathbf{H}(M ; \mathscr{D}(p))$, the amalgamation map $A: \mathbf{H}(M ; \mathscr{D}(p)) \rightarrow \mathscr{D}(E)$, and the cokernel for this amalgamation map denoted by $N \mathscr{D}(p)$.

Note that in the preceding two paragraphs we must use the semisimplicial definitions of stable pseudo-isotopy spaces $\mathscr{T}_{j}(E), \mathscr{D}_{j}(E)$, which are given in $[65, \S 5]$, if $E$ is not a smooth manifold. Likewise, these semisimplicial definitions must be used in the definitions of $\mathbf{H}(M ; \mathscr{T}(p))$ and $\mathbf{H}(M ; \mathscr{D}(p))$.

In the following two theorems, we let $M$ denote a real, complex, quaternionic, or Cayley complete hyperbolic manifold. We let $I:\left(\bigcup_{i} C_{i}\right) \cup\left(\bigcup_{i} S^{1}\right) \rightarrow$ $M$ denote the map of Theorem A.1 and $p^{\prime}: E^{\prime} \rightarrow\left(\bigcup_{i} C_{i}\right) \cup\left(\bigcup_{i} S^{1}\right)$ denote the 
pullback along $I$ of the fibration $p: E \rightarrow M$. Note that there is a canonical mapping $I^{*}: E^{\prime} \rightarrow E$ of fibrations that covers the map $I$. By using $p^{\prime}: E^{\prime} \rightarrow\left(\bigcup_{i} C_{i}\right) \cup\left(\bigcup_{i} S^{1}\right)$ in place of $p: E \rightarrow M$ in the preceding paragraphs, we can get the homology $\Omega$-spectra $\mathbf{H}\left(\left(\bigcup_{i} C_{i}\right) \cup\left(\bigcup_{i} S^{1}\right) ; \mathscr{T}\left(p^{\prime}\right)\right)$ and $\mathbf{H}\left(\left(\bigcup_{i} C_{i}\right) \cup\left(\bigcup_{i} S^{1}\right) ; \mathscr{D}\left(p^{\prime}\right)\right)$, the amalgamation maps

$$
A: \mathbf{H}\left(\left(\bigcup_{i} C_{i}\right) \cup\left(\bigcup_{i} S^{1}\right) ; \mathscr{T}\left(p^{\prime}\right)\right) \rightarrow \mathscr{T}\left(E^{\prime}\right)
$$

and

$$
A: \mathbf{H}\left(\left(\bigcup_{i} C_{i}\right) \cup\left(\bigcup_{i} S^{1}\right) ; \mathscr{D}\left(p^{\prime}\right)\right) \rightarrow \mathscr{D}\left(E^{\prime}\right),
$$

and the cokernel $\Omega$-spectra of these amalgamation maps denoted by $N \mathscr{T}\left(p^{\prime}\right)$ and $N \mathscr{D}\left(p^{\prime}\right)$.

Theorem A.18.1. Let $M$ be any real, complex, quaternionic, or Cayley complete hyperbolic manifold. The maps $I:\left(\bigcup_{i} C_{i}\right) \cup\left(\bigcup_{i} S^{1}\right) \rightarrow M$ and $I^{*}: E^{\prime} \rightarrow E$ induce the following equivalences of $\Omega$-spectra.

(a) $N \mathscr{T}\left(p^{\prime}\right) \cong N \mathscr{T}(p)$.

(b) $N \mathscr{D}\left(p^{\prime}\right) \cong N \mathscr{D}(p)$.

We remark that Theorem A.18.1 holds also for any Riemannian manifold $M$ having finite volume and whose sectional curvature values lie in an interval $\left(c_{1}, c_{2}\right)$ with $c_{1}<c_{2}<0$.

Theorem A.18.2. Let $M$ be any real, complete, hyperbolic manifold. Suppose that the homotopy fibration $p: E \rightarrow M$ has no holonomy up to homotopy. Then in the category of $\Omega$-spectra, each of the amalgamation maps $A: \mathbf{H}(M ; \mathscr{T}(p))$ $\rightarrow \mathscr{T}(E)$ and $A: \mathbf{H}(M ; \mathscr{D}(p)) \rightarrow \mathscr{D}(E)$ has a left inverse.

We remind the reader that the fibration $p: E \rightarrow M$ is said to have no holonomy up to homotopy if for any map $g: S^{1} \rightarrow M$ from the circle the pulled back fibration of $p: E \rightarrow M$ along $g$ is fiber homotopy equivalent to a product fibration over $S^{1}$. It seems likely to the authors that the holonomy hypothesis of Theorem A.18.2 is not necessary.

Recall that a group $\Gamma$ is called $K$-flat if $\mathrm{Wh}(\Gamma \times A)=0$ holds for all finitely generated free abelian groups $A$ (cf. [23, §0]).

Corollary A.18.3. Let $M$ be any real, complex, quaternionic, or Cayley complete hyperbolic manifold. Then $\pi_{1} M$ is $K$-flat.

Special cases of Theorems A.18.1 and A.18.2 have been announced in [22]. Corollary A.18.3 has been announced in [23]. There are versions of Theorems A.18.1 and A.18.2 where $p: E \rightarrow M$ is a stratified fibration over the hyperbolic orbifold $M$. Special cases of these orbifold versions of Theorems A.18.1 and 
A.18.2 were announced in [22]. The details will appear in later publications of the authors.

In the remainder of this appendix, we will give brief indications of the proofs of Theorems A.18.1, A.18.2 and Corollary A.18.3.

We will concentrate on the functor $\mathscr{D}()$ as the proofs are the same for the functor $\mathscr{T}()$. Let $X$ denote any codimension zero smooth submanifold of $M$, let $Y$ denote any open subset of $X$, and let $D$ denote any compact subset of $X$. For any $\varepsilon>0$, we let $\mathscr{D}(X ; Y, \varepsilon, D)$ denote the subset of all stable smooth pseudo-isotopies in $\mathscr{D}\left(p^{-1}(X)\right)$ that are $\varepsilon$-controlled over $Y$ (with respect to the projection $p: p^{-1}(X) \rightarrow X$ ) and whose support lies over the subset $D$ (again with respect to the projection $p: p^{-1}(X) \rightarrow X$ ). Denote by $C \mathscr{D}(X ; Y, D)$ the space of all paths $q:[0, \infty) \rightarrow \mathscr{D}\left(p^{-1}(X)\right)$ such that $q(t) \in \mathscr{D}\left(X ; Y,(t+1)^{-1}, D\right)$ holds for all $t \in[0, \infty)$. Define $C \mathscr{D}(X ; Y)$ to be the direct limit space $\lim _{D \rightarrow X} C \mathscr{D}(X ; Y, D)$. By applying the arguments given in [65] (see, in particular, [65, $\S 6$ and the appendix]) to the functor $\mathscr{D}()$, we can derive the following.
A.18.4.
(a) $C \mathscr{D}(X)$ is an $\Omega$-spectrum.
$($ Here $C \mathscr{D}(X)=C \mathscr{D}(X ; X)$.

(b) There is an equivalence of $\Omega$-spectra

$$
C \mathscr{D}(X) \cong \mathbf{H}\left(X ; \mathscr{D}\left(p \mid p^{-1}(X)\right)\right) .
$$

We can now consider the proof of Theorem A.18.1. We may assume that for each positive integer $n$ the restricted map $I_{n}=I \mid\left(\bigcup_{i}^{n} C_{i}\right) \cup\left(\bigcup_{i}^{n} S^{1}\right)$ is a smooth embedding. Let $T_{n}$ denote a tubular neighborhood of $\operatorname{Image}\left(I_{n}\right)$ in $M$ such that $T_{n} \subset T_{n+1}$ holds for all $n$. We consider the following commutative diagram of maps of $\Omega$-spectra:

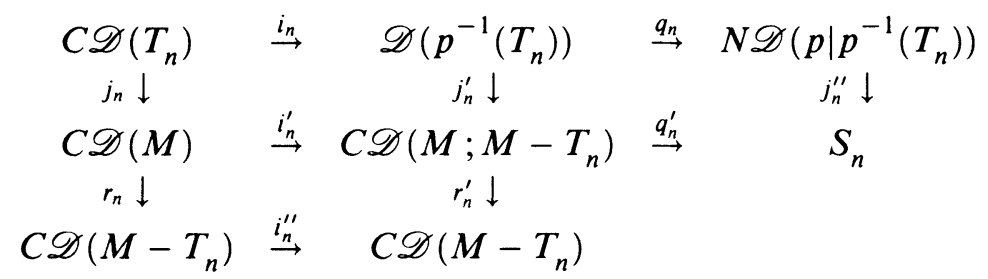

where all the maps $i_{n}, i_{n}^{\prime}, i_{n}^{\prime \prime}, j_{n}, j_{n}^{\prime}, j_{n}^{\prime \prime}$ are induced by inclusions, $r_{n}$ and $r_{n}^{\prime}$ are the restriction maps (see [65, $\S 6$ and the appendix]), and $q_{n}, q_{n}^{\prime}$ are the canonical maps to the cokernel $\Omega$-spectra for $i_{n}, i_{n}^{\prime}$. Note that the two left columns are fibrations and the top two rows are also fibrations in the category of $\Omega$-spectra. Note also that $i_{n}^{\prime \prime}$ is an equivalence of $\Omega$-spectra (in fact, it is the identity map). We conclude that $j_{n}^{\prime \prime}$ is an equivalence of $\Omega$-spectra. Let $N_{\infty}$ denote the direct limit $\Omega$-spectrum $\lim _{n \rightarrow \infty} N \mathscr{D}\left(p \mid p^{-1}\left(T_{n}\right)\right)$, and let $S_{\infty}$ denote the direct limit $\Omega$-spectrum $\lim _{n \rightarrow \infty} S_{n}$. Note that taking the direct limit of the maps $j_{n}^{\prime \prime}$ yields an equivalence $j_{\infty}^{\prime \prime}: N_{\infty} \rightarrow S_{\infty}$ of $\Omega$ spectra. On the other hand, it follows from a smooth fibered version of the 
argument in Step II of the proof of Theorem A.1 that the direct limit $\Omega$ spectrum $\lim _{n \rightarrow \infty} C \mathscr{D}\left(M ; M-T_{n}\right)$ is equivalent to the $\Omega$-spectrum $\mathscr{D}(E)$ via the inclusion induced map. From this last fact and from A.18.4, we conclude that $S_{\infty}$ is equivalent to $N \mathscr{D}(p)$. Moreover, $N_{\infty}$ is equivalent to the $\Omega$-spectrum $N \mathscr{D}\left(p^{\prime}\right)$. Thus, the equivalence $j_{\infty}^{\prime \prime}: N_{\infty} \rightarrow S_{\infty}$ is really the equivalence $N \mathscr{D}\left(p^{\prime}\right) \cong N \mathscr{D}(p)$ of Theorem A.18.1(b). This completes the outline for the proof of Theorem A.18.1.

We now consider the proof of Theorem A.18.2. Using a fibered version of the argument in Step I of the proof of Theorem A.2, we can argue that the following equivalence of $\Omega$-spectra exists.

A.18.5. $\mathscr{D}\left(p^{-1}\left(T_{n}\right)\right) \cong C \mathscr{D}\left(T_{n}\right) \times N \mathscr{D}\left(p \mid p^{-1}\left(T_{n}\right)\right)$.

(It is in carrying out the fibered version of Step I in the proof of Theorem A.2 that we use the holonomy hypothesis of A.18.2.) The equivalence of A.18.5 provides us with "inclusions" $g_{n}: N \mathscr{D}\left(p \mid p^{-1}\left(T_{n}\right)\right) \rightarrow \mathscr{D}\left(p^{-1}\left(T_{n}\right)\right)$. The direct limit of these inclusion maps is an "inclusion" $G: N \mathscr{D}\left(p^{\prime}\right) \rightarrow \mathscr{D}(E)$. Let $F: C \mathscr{D}(M) \rightarrow \mathscr{D}(E)$ denote the map that sends a path $q:[0, \infty) \rightarrow \mathscr{D}(E)$ (such paths represent points in $C \mathscr{D}(M)$ ) to the point $q(0)$ of $\mathscr{D}(E)$. We now have the direct sum map $F \oplus G: C \mathscr{D}(M) \times N \mathscr{D}\left(p^{\prime}\right) \rightarrow \mathscr{D}(E)$. By using Theorem A.18.1, A.18.4, and A.18.5, one can argue that $F \oplus G$ is the desired equivalence of $\Omega$-spectra in A.18.2. This completes our outline of the proof for Theorem A.18.2.

Finally, we can deduce Corollary A.18.3 from Theorem A.18.1 as follows. Let $A$ be any finitely generated free abelian group. We wish to show that $\mathrm{Wh}\left(\pi_{1} M \times A\right)=0$. Note that $A=\pi_{1} T^{n}$ for some $n$, where $T^{n}$ is the $n$ dimensional torus. In Theorem A.18.1, let $p: E \rightarrow M$ denote the projection of $E=M \times T^{n}$ onto its first factor. We must show that $\pi_{1}\left(\mathscr{T}_{0}(E)\right)=0$. Since $\mathbf{H}_{0}(M ; \mathscr{T}(p)) \rightarrow \mathscr{T}_{0}(E) \rightarrow N \mathscr{T}_{0}(p)$ is a homotopy fibration, it will suffice to show that $\pi_{1}\left(\mathbf{H}_{0}(M ; \mathscr{T}(p))\right)=0$ and $\pi_{1}\left(N \mathscr{T}_{0}(p)\right)=0$. There is a spectral sequence beginning with terms of the form $H_{j}\left(M ; \pi_{k}\left(\mathscr{T}_{0}\left(T^{n}\right)\right)\right)$ and abutting to $\pi_{j+k}\left(\mathbf{H}_{0}(M ; \mathscr{T}(p))\right)$ (see [65, Theorem 8.7]). Since $\pi_{k}\left(\mathscr{T}_{0}\left(T^{n}\right)\right)=0$ for $k=0,1$ (cf. [19]), it follows that $\pi_{1}\left(\mathbf{H}_{0}(M ; \mathscr{T}(p))\right)=0$. Note that by Theorem A.18.1 we have that $\pi_{1}\left(N \mathscr{T}_{0}(p)\right)=\pi_{1}\left(N \mathscr{T}_{0}\left(p^{\prime}\right)\right)$. Moreover, there is the homotopy fibration

$$
\mathbf{H}_{0}\left(\left(\bigcup_{i} C_{i}\right) \cup\left(\bigcup_{i} S^{1}\right) ; \mathscr{T}\left(p^{\prime}\right)\right) \rightarrow \mathscr{T}_{0}\left(E^{\prime}\right) \rightarrow N \mathscr{T}_{0}\left(p^{\prime}\right) .
$$

So to show that $\pi_{1}\left(N \mathscr{T}_{0}(p)\right)=0$, it will suffice to verify the following:

(i) $\pi_{1}\left(\mathscr{T}_{0}\left(E^{\prime}\right)\right)=0$.

(ii) $\pi_{0}\left(\mathbf{H}_{0}\left(\left(\bigcup_{i} C_{i}\right) \cup\left(\bigcup_{i} S^{1}\right) ; \mathscr{T}\left(p^{\prime}\right)\right)=0\right.$.

Since each $C_{i}$, and $S^{1}$, is a compact closed aspherical manifold with virtually poly- $Z$ fundamental group equality, (i) follows from [19]. (ii) follows from [19] and a spectral sequence argument. 


\section{REFERENCES}

1. D. R. Anderson and W.-c. Hsiang, The functors $K_{-i}$ and pseudo-isotopies of polyhedra, Ann. of Math (2) 105 (1977), 201-223.

2. D. V. Anosov, Geodesic flows on closed Riemannian manifolds with negative curvature, Proc. Steklov Inst. Math., Vol. 90, Amer. Math. Soc., Providence, RI, 1969.

3. W. Ballmann, M. Gromov, and V. Schroeder, Manifolds of nonpositive curvature, Birkhäuser, Boston, 1985.

4. R. L. Bishop and B. O'Neill, Manifolds of negative curvature, Trans. Amer. Math. Soc. 145 (1969), 1-49.

5. A. Borel, Stable real cohomology of arithmetic groups, Ann. Sci. École Norm. Sup. (4) 7 (1974), 235-272.

6. W. Browder and F. Quinn, A surgery theory for G-manifolds and stratified sets, ManifoldsTokyo 1973, Univ. of Tokyo Press, Tokyo, 1975, pp. 27-36.

7. D. Burghelea and R. Lashof, The homotopy type of the space of diffeomorphisms. I, Trans. Amer. Math. Soc. 196 (1975), 1-36.

8. _ The homotopy type of the space of diffeomorphisms. II, Trans. Amer. Math. Soc. 196 (1975), 37-50.

9. - Stability of concordances and suspension homeomorphism, Ann. of Math. (2) 105 (1977), 449-472.

10. D. Burghelea, Automorphisms of manifolds, Proc. Sympos. Pure Math., Vol. 32, Amer. Math. Soc., Providence, RI, 1978, pp. 347-371.

11. T. A. Chapman and S. Ferry, Approximating homotopy equivalences by homeomorphisms, Amer. J. Math. 101 (1979), 567-582.

12. D. Epstein and M. Shub, Expanding endomorphisms of flat manifolds, Topology 7 (1968), 139-141.

13. F. T. Farrell, The obstruction to fibering a manifold over a circle, Indiana Univ. Math. J. 21 (1971), 315-346.

14. F. T. Farrell and W.-c. Hsiang, Manifolds with $\pi_{1}=G \times_{\alpha} T$, Amer. J. Math. 95 (1973), 813-848.

15. __ Rational L-groups of Bieberbach groups, Comment. Math. Helv. 52 (1977), 89-109.

16. __ The topological-Euclidean space form problem, Invent. Math. 45 (1978), 175-179.

17. _ On the rational homotopy groups of the diffeomorphism groups of discs, spheres and aspherical manifolds, Proc. Sympos. Pure Math., Vol. 32, Amer. Math. Soc., Providence, RI, pp. 325-337.

18. __ On Novikov's conjecture for non-positively curved manifolds, I, Ann. of Math. (2) 113 (1981), 199-209.

19. $\ldots$, The Whitehead group of poly-(finite or cyclic) groups, J. London Math. Soc. (2) 24 (1981), 308-324.

20. _ The stable topological-hyperbolic space form problem for finite volume manifolds, Invent. Math. 69 (1982), 155-170.

21. _ Topological characterization of flat and almost flat Riemannian manifolds $M^{n} \quad(n \neq$ 3, 4), Amer. J. Math. 105 (1983), 641-672.

22. F. T. Farrell and L. E. Jones, Algebraic $K$-theory of spaces stratified fibred over hyperbolic orbifolds, Proc. Nat. Acad. Sci. U.S.A 83 (1986), 5364-5366.

23. _ K K-theory and dynamics. I, Ann. of Math (2) 124 (1986), 531-569.

24. __ Algebraic K-theory of discrete subgroups of Lie groups, Proc. Nat. Acad. Sci. U.S.A. 84 (1987), 3095-3096.

25. __ K-theory and dynamics. II, Ann. of Math. (2) 126 (1987), 451-493.

26. _ The surgery L-groups of poly-(finite or cyclic) groups, Invent. Math. 91 (1988), 559-586. 
27. __, Topological rigidity for hyperbolic manifolds, Bull. Amer. Math. Soc. 19 (1988), 277-282.

28. __ Foliated control theory I, $K$-theory 2 (1988), 357-399.

29. __ Foliated control theory II, $K$-theory 2 (1988), 401-430.

30. __ Splitting over flat and almost flat Riemannian manifolds (in preparation).

31. M. Freedman and F. Quinn, 4-manifolds, Ann. of Math. Stud. (to appear).

32. M. Gromov, Manifolds of negative curvature, J. Differential Geom. 13 (1978), 223-230.

33. A. E. Hatcher, Concordance spaces, higher simple homotopy theory, and applications, Proc. Sympos. Pure Math., Vol. 32, Amer. Math. Soc., Providence, RI, 1978, pp. 3-21.

34. A. Hatcher and J. Wagoner, Pseudo-isotopies of compact manifolds, Astérisque 6 (1973).

35. N. J. Hicks, Notes on differential geometry, van Nostrand, Princeton, 1965.

36. M. W. Hirsch, Immersions of manifolds, Trans. Amer. Math. Soc. 93 (1959), 242-276.

37. K. Igusa, The stability theorem for pseudoisotopies, $K$-theory 2 (1988), 1-355.

38. L. E. Jones, Patch spaces, Ann. of Math. (2) 97 (1973), 306-343.

39. __ The non-simply connected characteristic variety theorem, Proc. Sympos. Pure Math., Vol. 32, Amer. Math. Soc., Providence, RI, 1978, pp. 131-140.

40. __ Construction of surgery problems, Geometric Topology (J. Cantrell, ed.), Academic Press, NY, 1979, pp. 367-391.

41. G. G. Kasparov, Topological invariants of elliptic operators. I. K-homology, Izv. Akad. Nauk SSSR Ser Mat. 39 (1975), 796-838.

42. __ Equivariant KK-theory and the Novikov conjecture, Invent. Math. 91 (1988), 147-201.

43. R. C. Kirby and L. C. Siebenmann, Foundational essays on topological manifolds, smoothings and triangulations, Ann. of Math. Stud., Vol. 88, Princeton Univ. Press, Princeton, NJ, 1977.

44. H. Kneser, Geschlossene Flächen in dreidimensionalen Mannigfalligkeiten, Jahresber. Deutsch. Math.-Verein. 38 (1929), 248-260.

45. N. Levitt, Poincaré duality cobordism, Ann. of Math. (2) 96 (1972), 211-244.

46. S. Maumary, Proper surgery groups and Wall-Novikov groups, Algebraic K-theory. III, Lecture Notes in Math., Vol. 343, Springer, Berlin, 1973, pp. 526-539.

47. J. Milnor, A unique factorization theorem for 3-manifolds, Amer. J. Math. 84 (1962), 1-7.

48. __ On fundamental groups of complete affinely flat manifolds, Adv. in Math. 25 (1977), 178-187.

49. A. S. Mishchenko, Infinite dimensional representations of discrete groups and higher signatures, Izv. Akad. Nauk SSSR Ser. Mat. 38 (1974), 81-106.

50. G. D. Mostow, Quasi-conformal mappings in $n$-space and the rigidity of hyperbolic space forms, Inst. Hautes Études Sci. Publ. Math. 34 (1967), 53-104.

51. __ Strong rigidity of locally symmetric spaces, Ann. of Math. Stud., Vol. 78, Princeton Univ. Press, Princeton, NJ, 1973.

52. A. J. Nicas, Induction theorems for groups of homotopy manifold structures, Mem. Amer. Math. Soc., no. $267,1982$.

53. __ An infinite family of non-Haken hyperbolic three-manifolds with vanishing Whitehead groups, Math. Proc. Cambridge Philos. Soc. 99 (1986), 239-246.

54. A. J. Nicas and C. W. Stark, Whitehead groups of certain hyperbolic manifolds, Math. Proc. Cambridge Philos. Soc. 95 (1984), 299-308.

55. __ Whitehead groups of certain hyperbolic manifolds II, Combinatorial Group Theory and Topology, Ann. of Math. Studies, Vol. 111, Princeton Univ. Press, Princeton, NJ, 1987, pp. 415-432.

56. G. Prasad, Strong rigidity of Q-rank 1 lattices, Invent. Math. 21 (1973), 255-286.

57. F. Quinn, A geometric formulation of surgery, Ph.D. thesis, Princeton University, 1969.

58. __ A geometric formulation of surgery, Topology of Manifolds, Markham, Chicago, 1970, pp. $500-511$. 
59. __ Ends of maps I, Ann. of Math. 110 (1979), 275-331.

60. A. Ranicki, Algebraic L-theory II: Laurent extensions, Proc. London Math. Soc. (3) 27 (1973), 126-158.

61. L. C. Siebenmann, The obstruction to finding a boundary for an open manifold of dimension $>5$, Ph.D. thesis, Princeton Univ., 1965.

62. L. Taylor, Surgery on paracompact manifolds, Ph.D. thesis, Univ. of California at Berkeley, 1972.

63. F. Waldhausen, Algebraic K-theory of topological spaces I, Proc. Sympos. Pure Math., Vol. 32, Amer. Math. Soc., Providence, RI, 1978, pp. 35-60.

64. C. T. C. Wall, Surgery on compact manifolds, Academic Press, London, 1971.

65. F. Quinn, Ends of maps II, Invent. Math. 68 (1982), 353-424.

66. F. T. Farrell and L. E. Jones, Compact negatively curved manifolds (of $\operatorname{dim} \neq 3,4$ ) are topologically rigid, Proc. Nat. Acad. Sci. U.S.A. (to appear).

67. __ Negatively curved manifolds with exotic smooth structures (submitted for publication).

Department of Mathematics, Columbia University, New York, New York 10027

Department of Mathematics, SUNY at Stony Brook, Stony Brook, New York 11794 